Netwit? $+41+10$
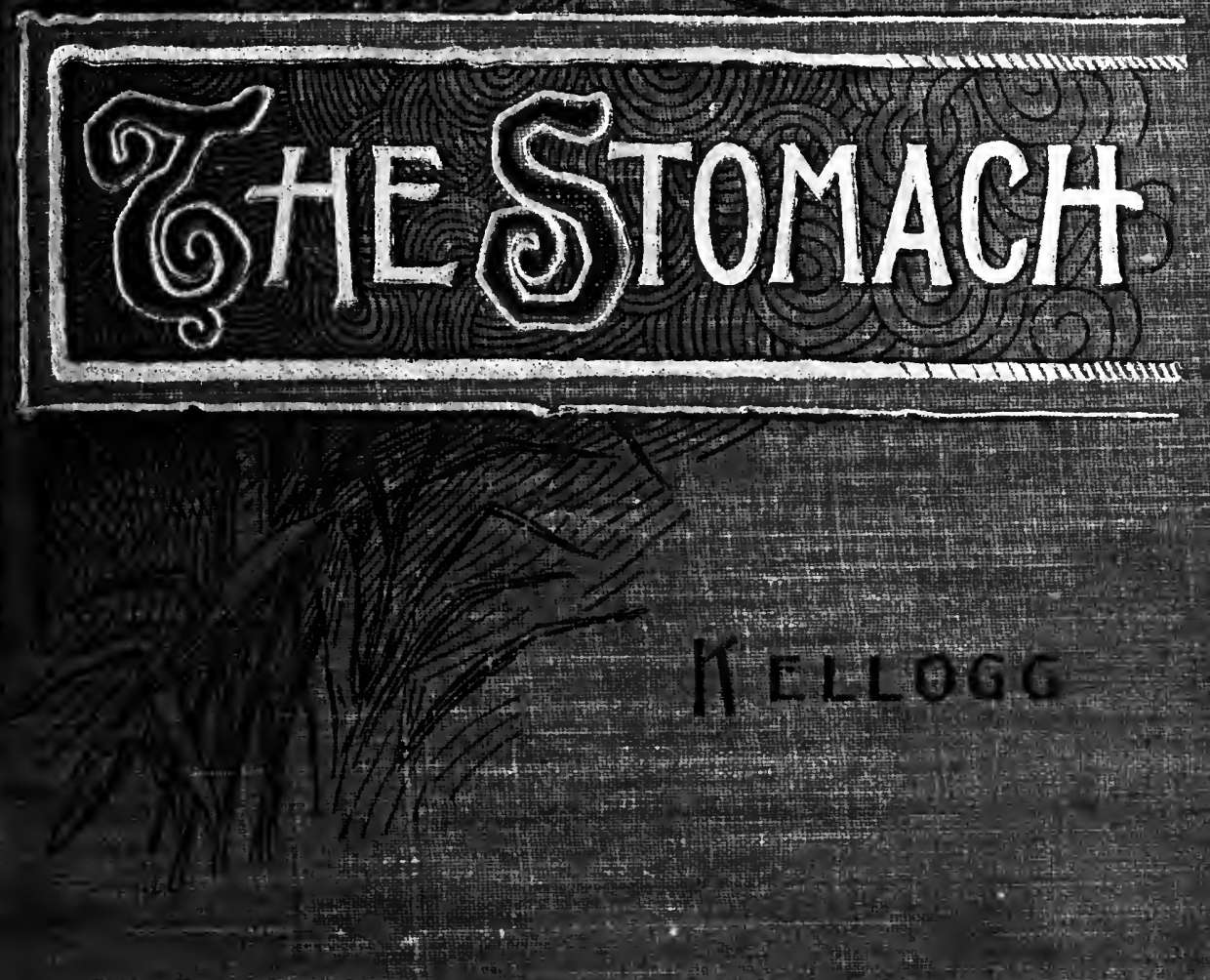


$$
\begin{aligned}
& \text { Dr ILCouter - } \\
& \text { Bethereck- } \\
& \text { gu? } 16 \text { - }
\end{aligned}
$$




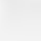




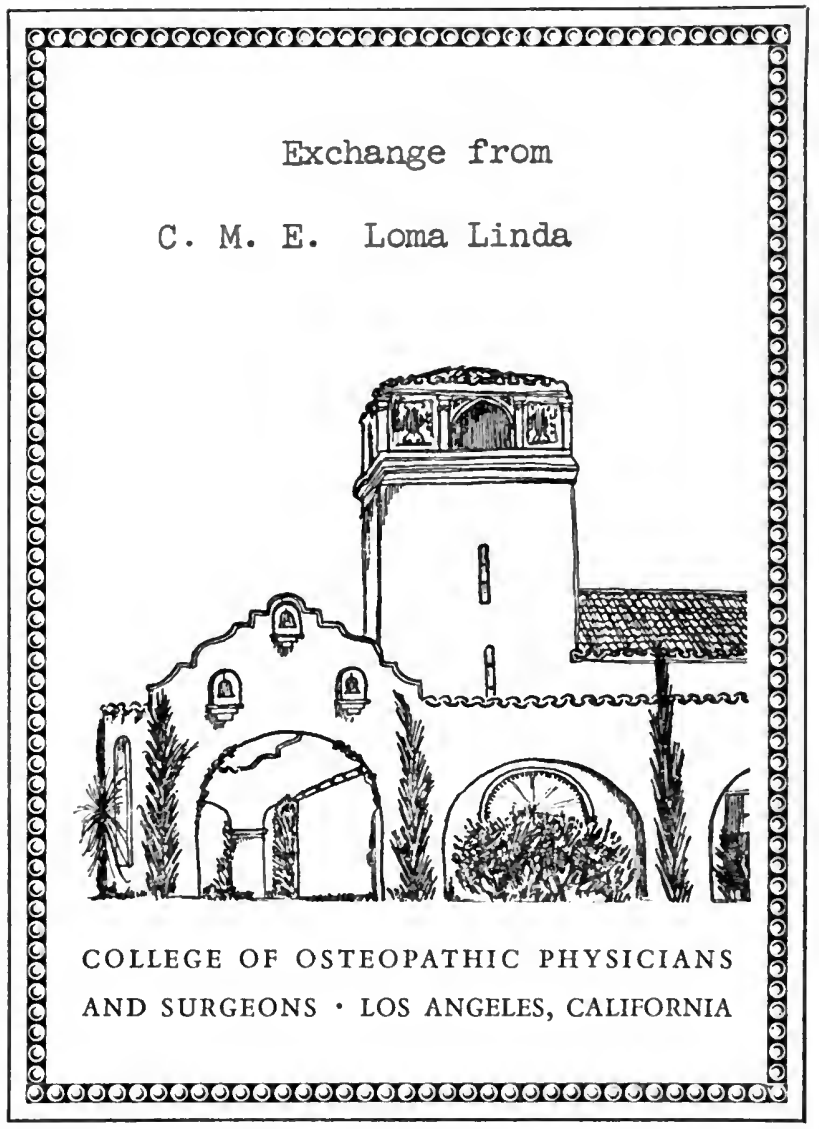




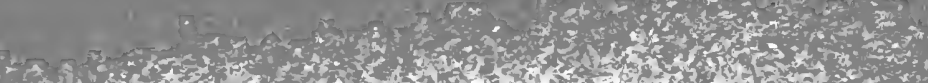

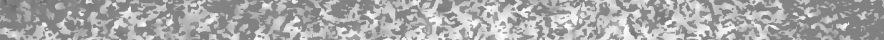

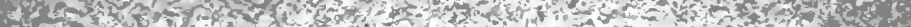

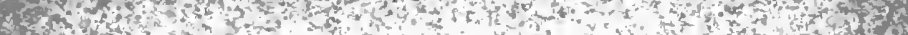

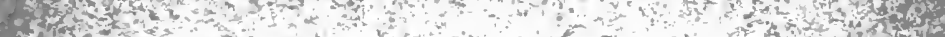

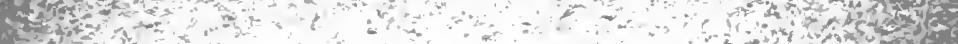
in

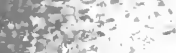

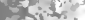

Ateme

$+10$

cotitis.

vich

s. 35

1. $3<$.

* 40 inio

a)

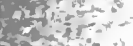

7325

$+3 x+5 ; x$

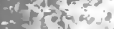

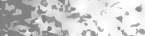

tothon.

7. 30 a

jes $2 \pi$

int?

$3=$

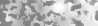

3120

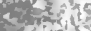

sini

군다.

$x^{2} \log ^{2}$

if $\geq 2=0$

$\rightarrow$

$+5$

- 5 >z

- restivis

$2 x^{2}=1$

soti

$x^{2}=v^{3}$

$\sqrt{3} x^{2}-2$

$x_{0}=2$

4 दि -1

the

ind $=0$

sert

$=1-3+a^{2}$

$5 x^{2}=1 ; 1$

aces?

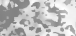

$3 x^{2} y$

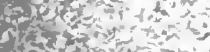

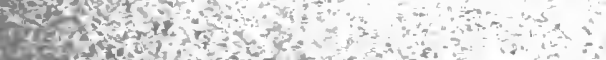

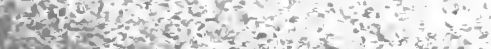

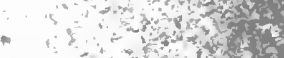

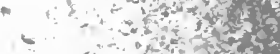

$x^{2}=2 x+3 x^{2}+2$

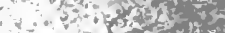

$\sin ^{2}+x^{2}+x^{2}$ ?

$+10+3$ a

Fo-3hats

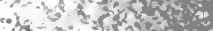

as $-3 x^{2}$

7 and of

$\therefore=23^{2}=$

$=-x+420$

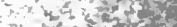

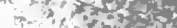

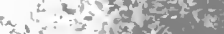

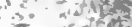

- T-

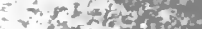

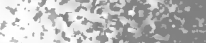

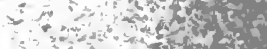

$\therefore$ a d

$404 x^{2}+4$

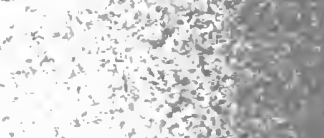

it $x+2 x+2$

$\therefore-10^{2}$

$2^{2}+302$

$\therefore x+2=0$

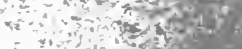

$=x^{2}=x^{2}-3$

$\therefore=7-4.46$

$x-2 y-500$

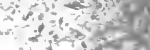

atise

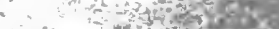

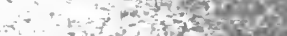

$\therefore=$

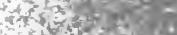

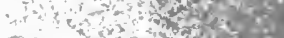

"a "

$\rightarrow x^{2}-x^{2}=x^{2}$

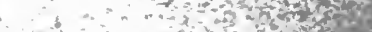

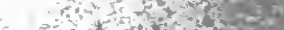

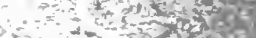

$\rightarrow \quad 2+2 x^{\circ}$

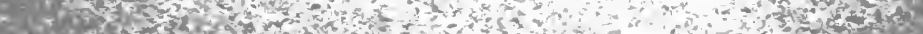

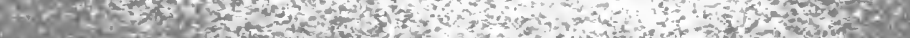

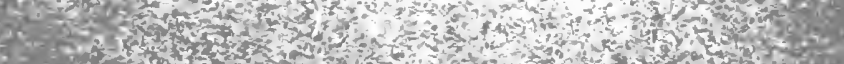

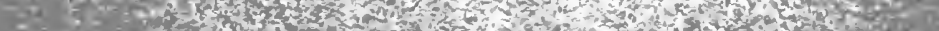

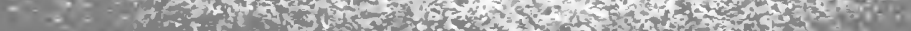

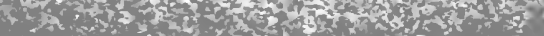




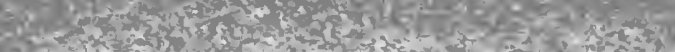

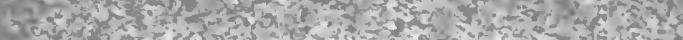

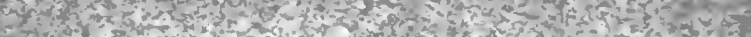

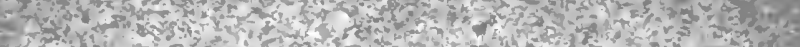

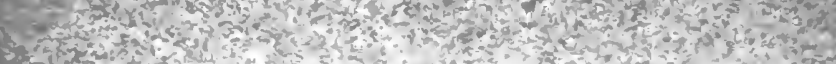

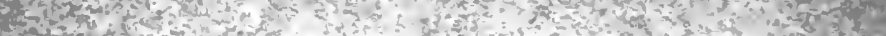

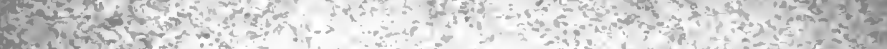

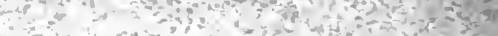

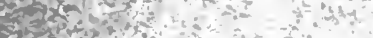

$x \rightarrow 3, x+2,90$

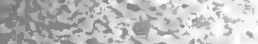

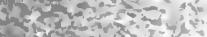

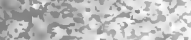

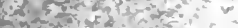

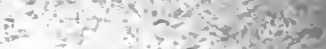

$3+2-2=0$ in

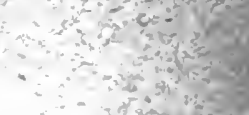

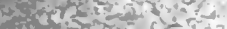

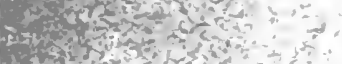

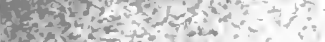

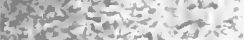

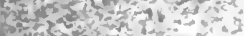

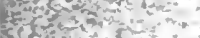

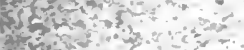

Af

sisititi:

$\log _{2}>x^{2}$

a

ing.

(5,

3its?

1.

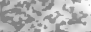

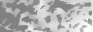

sing is

कon

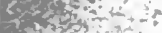

Thess $32-3$

$x^{2}+2=-5 i$

ateris?

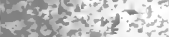

$3 x^{2}-2,2$

xis

toring,

क्ये

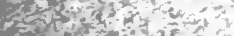

$\tan x=23$

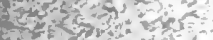

$3+7=2-14$

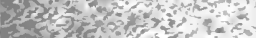

3efar

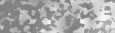

Setent $=0$

$2+\operatorname{la}^{2} 6$

wisters

$-4,2<x$

stiplos

$7 x^{2} x^{2}+3$

reter $=0 \%$.

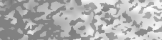

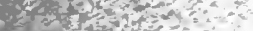

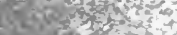

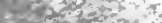

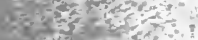

ir a cosing

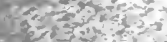

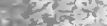

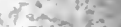

$\because: 35<2$

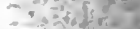

$\left.\because c^{2}\right)^{2}=$

$0 x+4 x^{2}-4$

$\therefore-i^{2}+x^{2}=$

$-3=4+2 x+\frac{1}{10}$

$\lim ^{2} x^{2}-1$

$\because \frac{100}{62}$

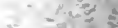

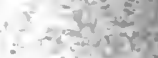

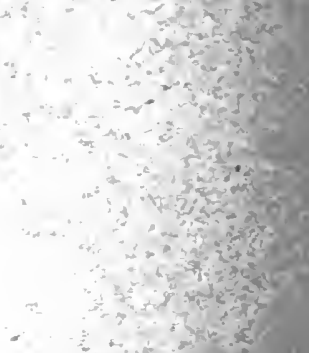

$\frac{y^{2}}{7+2}+x^{2}$

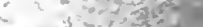

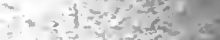

$a^{2}+x^{2}+x^{2}$

a से

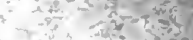

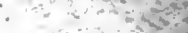

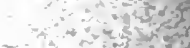

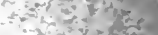

$\therefore x+y=3$

$\therefore-3-2$

$4-25-21$

$2 \pi y, 10 x^{2}$

$a^{2 m}-3^{3}-h=$

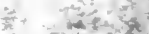

the?

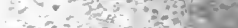

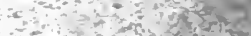

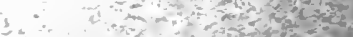

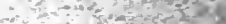

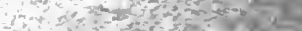

is

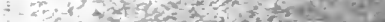

cisis

Novist

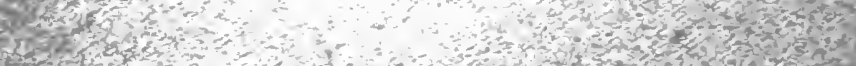

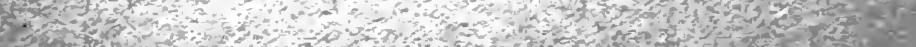

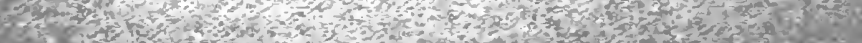

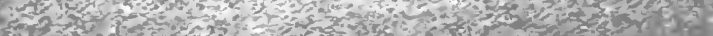

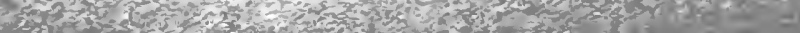




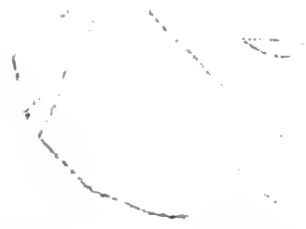

Digitized by the Internet Archive in 2007 with funding from Microsoft Corporation

http://www.archive.org/details/stomachitsdisord00kelliala 


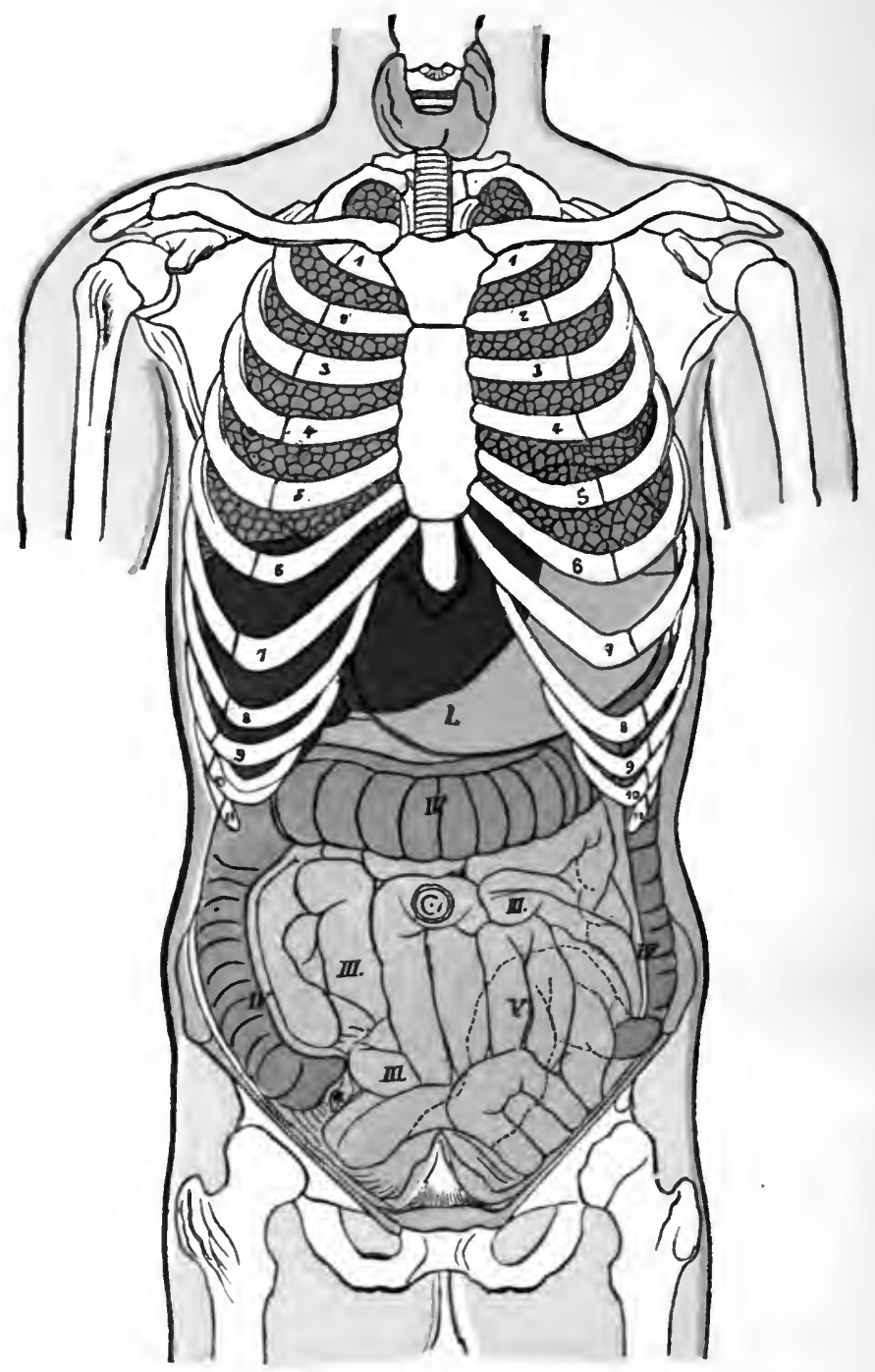

In the above diagram the position of the stomach will be clearly seen, together with its relation to other internal organs. 1. Stomach, partially covered by the liver and lungs: III. Small intestines; IV. Colon; V. Lower or sigmoid portion of the colon.

$$
\text { PLATE I. - INTERNAL ORGANS. }
$$




\section{THE STOMACH:}

ITS DISORDERS, AND

\section{HOW TO CURE THEM.}

-

BY J. H. KELLOGG, M. D.,

President of the Medical Missionary College, Chicago, Ill.; Member of the Brit. ish Gynecological Society; International Periodical Congress of Gynecology and Obstetrics; British and American Associations for the Advancement of Science; the Socicte d'Hygiène of France; Pan-American Congress of Religion and Education; American Society of Mioroscopists; American Electro-Therapeutic Association; American Climatological Society; American Medical Association; Mississippi Valley Medical Association; Tri-State Medical Society;

Michigan Slate Medical Society; Superintendent of the Battle Creek (Mich.) Sanitarium.

ILLUSTRATED.

MODERN MEDICINE PUB. CO., BATTLE CREEK, MICH.

Chicago:

NEW YORK :

LONDON.

1896. 


$$
\begin{aligned}
& W I_{1} 113 \\
& K 295 \\
& 1.896
\end{aligned}
$$

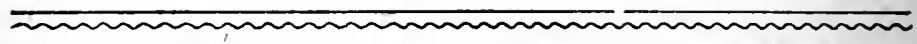

Entered according to Act of Congress, in the year 1896, BY J. H. KELLOGG, M. D.,

In the office of the Librarian of Congress, at Washington, D. C.

ALL RIGHTS RESERVED.

Entered also at Stationers' Hall, London, England.

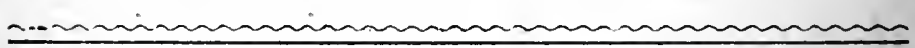




\title{
To
}

\section{MY PATIENTS}

\author{
This Book
}

Is

Affectionately Dedicated 



\section{PREFACE.}

Av eminent comparative anatomist defines an animal as "a stomach with various organs appended," - a definition which presents in a very clear light the relative importance of the stomach in the association of the organs and structures which constitute the human as well as the animal body. The stomach must certainly be regarded as the center of the nutritive processes of the body, and any derangement of its functions must therefore result in disorder of the entire organism.

The close relation between the condition of the stomach and that of the mind has been observed even by the most primitive nations, and has led some of them to regard the stomach as the veritable seat of the sonl. There is sound truth in the ancient German adage, "As a man eateth, so is he."

It may truly be said that disorders of digestion are the most prevalent of all human ills, and investigators within the last twenty years have been making more and more clear the fact that the great majority of diseases are primarily due to derangement of the digestive processes. The remarkable researches of Professor Bouchard, of Paris, and others, lave shown that such discases as consumption, typhoid lever, and cholera, as well as Bright's clisease and analogous maladies, are indirectly, if not directly, due to a disordered condition of the stomach, as the result of which the defenses of the body are broken down, and infection rendered possible.

Notwithstanding the great prevalence of stomach disorders, and the large amount of knowledge and experience which have been accumulated by the profession in regard to them within the last few years, it is nevertheless true that no other class of curable disorders has more frequently baffled the best directed efforts of medical practitioners. This is doubtless owing to the imperfect knowledge of the processes of digestion and of the nature of the disorders to which the digestive organs are subject which has prevailed from the earliest times to within a comparatively brief period. Within the last score of years, 
however, marvelous progress has been made in the study of the stomach and its work, through the use of the stomach-tube, and by the aid of experimental researches undertaken by numerous European and a few American investigators.

As the result of these inquiries, a great many most interesting and important facts have been discovered, which have almost wholly revolutionized our ideas respecting the nature of digestive ailments and successful methods of treatment.

One of the leading purposes of this book is to call attention to these newer facts in relation to the digestive processes, and especially to point out their relation to the prevention and successful treatment of dyspepsia and allied ailments. As will be seen by reference to the table of contents, the work embodies, first, a bricf sketch of the processes of digestion; then a consideration of the causes of indigestion, and their bearing upon dietetic and other habits, to which is added a description of the various symptoms present in functional diseases of the stomach, together with the means by which they may be relieved, followed by a consideration of each of the several classes of digestive disorders, and the proper dietetic and other measures necessary for their cure.

In the study of the practical part of the work, it will be noticed that the measures of treatment recommended are of a remarkably simple character. This may lead to the impression that they are lacking in efficiency; hence it may be proper to say in their defense, that many years of experience in the treatment of a large number of patients of this class, in connection with the Battle Creek (Mich.) Sanitarium, have fully established the author in the belief that in the proper regulation of the diet, in the varied uses of water as described in scientiflc hydrotherapy, in the employment of electricity, Swedish movements, Swedish gymnastics, massage, and exercise, together, with general control of the habits of life, we have means of far greater value than all others for combating those causes which give rise to indigestion, and of aiding nature in her efforts for recovery from the varied morbid conditions resulting from the numerous disorders commonly included under the general term, "dyspepsia."

The man who is suffering from indigestion is sick, not in his stomach alone, but in every cell and fiber of his body; there is no soundness in him. The patient, as well as his stomach, must 
receive attention. Our aim must be, not merely to cure the patient's symptoms, but to cure the patient himself. When this is done, the symptoms disappear.

Care has been taken to give an accurate and precise description of the various measures of treatment suggested, and to present them in such a manner that they may be readily available to every reader; but to save the consumption of space which would be necessitated by a constant repetition of the description, the treatments are referred to by number. The numbered paragraphs will be found in the chapter between pages 287 and 344 , in which are described all the various measures of treatment suggested in this work.

The description of the newer methods of investigating disorders of the stomach, whereby it is now possible to apply to their study the exact methods of chemistry and bacteriology, will, it is hoped, be of special interest to the reader. It is indeed not saying too much to assert that the nature of disorders of digestion may now be determined with as much accuracy as that of any other class of maladies, and that the old method of ascertaining by experiment the diet best adapted to an individual case, is no longer necessary,- at least where there are at command the resources afforded by recent progress in this branch of medical science.

The individual who wishes to make use of this work as a means to his own recovery, must, first of all, carefully read the entire work. He should then seek, either with or without the aid of a physician, to arrive at a correct diagnosis of his case. If possible, the services-of a skilled physician should be employed, a test meal taken, and the methods of examination described on pages 134, 320, and 352, utilized. More light is thrown upon the nature of digestive disturbances by the methods of examination here referred to, than from all other sources combined. The nature of the disorder being determined, it will be easy, by the aid of the table of contents or the index, to turn to the section which deals with this particular form of digestive disturbance; and then, having become familiar with the causes of the trouble, and the means of preventing its aggravation, such rational methods of treatment as will be efficient, and, it is believed, successful in a majority of cases, may be carried out at home.

In conclusion, the author desires to remark that it is not the purpose of this book to undertake to displace the wise family 
physician, but rather to aid and abet him in the management of a class of maladies which requires, perhaps more than all others, the thorough co-operation of the patient. That this may be secured, it is necessary that the patient should be educated in relation to the nature of his malady and the various methods by which he may render assistance in his own restoration to health. The need of such a work has been made apparent to the writer many times in his own practise. For years past, his patients have been constantly demanding a manual which should constitute, to some degree at least, a guide to them in their efforts to recover a healthy digestion; and it is hoped that this little work will be found to give the information which every sufferer from indigestion ought to have, and which every busy general practitioner cannot but be glad to have his patients suffering from this class of disorders. possess.

A number of cuts, some of which are original, and several plates, have been added to the text, which it is hoped will aid in giving the reader a clear idea of the subject presented.

J. $\mathbf{H}$. k.

Battle Creel, Mirh. 


\section{TABLE OF CONTENTS.}

PAQE.

THE STOMACH: ITS DISORDERS AND HOW TO CURE THEM

Modern researches - Germs and indigestion - Pepsin and patent medicines - Relation of dyspepsia to insanity and other disorders.

\section{THE ORGANS OF DIGESTION}

The healthy stomach - The alimentary canal - The mouth - The saliva - Ptyalin - The teeth - Temporaly teeth - Permanent teeth - The esophagus - The stomach - The peptic glands - 'The gastric juice - Pepsin - Rennin - The pylorus - The small intestine The intestinai juice - The absorbents - The colonThe liver - The bile - The pancreas - The pancreatic juice - Amylopsin - Trypsin - Steapsin - Milk-curdling ferment.

FOODS

Animal and vegetable foods - Plants the only food-producers - Food elements - Classification of food elements - Starch - Sugar - Glucose - Milk sugar - Albumen Casein - Fats - Salts - Indigestible elements - Condiments - Food substances - Foods of animal origin Milk - Cheese - Eggs - Flesh - Fish and Fowl - Shellfish - Oysters - Frogs - Lobsters - Salted and smoked meats - Vegetable foods - Fruits, grains, and vegetables - Legumes - Peptogens - Gluten - Dextrin - The natural diet of man - Vegetarianism - Uses of the several food elements - Digestibility of various foods - Nutritive values of common food substances.

THE DIGESTIVE FLUIDS . . . . .

Alexis St. Martin - What the saliva digests - What the gastric juice digests - What the bile digests - What the pancreatic juice digests - What the intestinal juice digests.

GENERAL VIEW OF THE DIGESTIVE PROCESS

Mastication - Salivary digestion - Deglutition, or swallowing - Stomach digestion - Action of the pylorus- 
Intestinal digestion - Other uses of the digestive fluids Gastric juice and bile, antiseptics - Peristaltic action - Absorption - How the diaphragm aids absorption The lacteals - The portal vein - Liver digestion - Gly: cogen - Liver starch - The two doors, the liver and the kidneys - Breathing and liver action - Vagaries of digestion - Poison found in oysters.

\section{THE MALADIES OF THE MODERN STOMACH}

The causes of dyspepsia - Conditions of healthy digestion-Causes of fermentation in the stomach-Disinfecting action of the gastric juice - Errors in diet Hasty eating - Experiments in relation to salivary digestion-Indigestion of starch - Relation of maltose - to digestion - Diseased teeth - Relation of defective teeth to indigestion - Microbes of the mouth-Disinfection of the mouth - Giles Corey's dream - Tartar of the teeth - Drinking at meals - Effects of ice-water-Dr. Beaumont's experiments upon Alexis St. Martin - Eating too frequently - All-gone feeling in the stomach - Eating between meals - Irregularity of meals - Rhythmical action of the intestines - Proper number of meals Habits of ancient nations in relation to the number of meals - Habits of modern nations in relation to the number of meals - Opinion of Dujardin-Beaumetz-Late suppers and six-o'clock dinners - Eating when exhausted - Effects of violent exercise upon the digestion - Rest before and after meals - Sleeping after meals-Too many varieties of food at one meal - Good and bad combinations of food - Hot and cold bathing after mealsErrors in quantity of food - Overeating - An extriordinary case - A case of dilatation of the stomach - Eating too little - How much should a person eat ? - Regulation of diet - Muscle-workers and brain-workers - Dietetic habits of Dickens and Newton - The quantity of food required at different ages - Desserts - Cornaro Experiments of Létheby and Parkes - Diet of pugilists in training - Economy in diet - Deflciency in necessary food elements - Effects of an impoverished diet - "Patent flour" and "family flour"- Unwholesome graham flour - Bogus gluten flours - Table relating to indigestion of food elements - Table showing proportion of nitrogenous to carbonaceous elements - Proper proportion of nitrogenous and carbonaceous elements - Amount 
of food required for a day's rations - Quality of food Bad cookely - Fried food - Uncooked food - Cooking, a sort of artificial ripening of food elements - Why green fruit and raw vegetables are indigestible - Imperfectly cooked food - Decayed foods - Sliell-fish - Rapid decay of animal tissues after death - The true nature of beef tea - Cheese and cholera morbus - Microbes of milk Soft food - Influence of soft food upon the teeth of cows and human beings - Too abundant use of fats - Decomposition of fats in the stomach - Why butter is indigestible - Why fats cause biliousness - Why fats are rendered indigestible by cooking - Why fats interfere with the digestion of other foods - The superior dietetic value of nuts - Nut butter - The excessive use of sugar - Relation of sugar to fermentation - Influence of sugar upon the stomach and liver - Why the excessive use of sugar causes diabetes and obesity - Excessive use of flesh food - Relation of meat diet to rheumatism, gout, and Bright's disease - Danger in the use of raw meat-Condiments - Beaumont's observations of the effects of condiments upon St. Martin - The excessive use of mustard and pepper in Mexico, and the results - Effects of salt upon digestion - Pickles - Vinegar - Vinegar eels Tinegar eels as parasites - Tea and coffee-Injurious effects of tea and coffee upon digestion-Alcohol-Sir William Roberts's exper'ments-Beaumont's experiments - Tobacco - Influence of tobacco upon digestionHard water - Alkalies - Baking-powders - Perverted appetites - Adulterations of food - Unseasonable diet Pressure upon the stomach - Brain work - Lack of exercise - Mental impressions-Drugs - Laxatives - Purgatives - Mineral waters - Sexual abuses-Diseases of other organs - Inherited dyspepsia - Nursing bottles Infant foods - Paregoric - Mrs. Winslow's Soothing Sirup - Worm teas - Dilatation of the stomach in children - Undetermined causes - Impure water - Germs in water.

\section{IMPORTANT NEW DISCOVERIES RELATING TO} DIGESTION

The stomach-tube - Measuring the size of the stomach -Clapotement-The author's method-Prolapse of stomach, kidneys, and other organs - Examination of stomach contents-Test meal-Facts determined by 
test meal-Bacteriological examination of the stomach contents - Germs of the stomach - Coefficients - Classification of stomach disorders - Hyperpepsia - Hypopepsia - Apepsia - Simple dyspepsia.

\section{SYMPTOMS OF DYSPEPSIA}

Classification of indigestions - Principal symptoms of indigestion - Symptoms pertaining to the mouth Symptoms pertaining to the throat - Symptoms pertaining to the esophagus - Symptoms pertaining to the stomach - Disturbances of the motor functions-The discharges - Symptoms pertaining to the rectum General nervous symptoms - Physical signs.

THE SYMPTOMATIC TREATMENT OF INDIGESTION

Uneasiness at the stomach - Flatulence - Fermentation - Acidity-Acidity from hyperpepsia-HeartburnWater-brash, or pyrosis - Nausea-Vomiting - Regurgitation, rumination, or merycism - Gripes, or colic Weight, constriction, tightness, etc.-Pain - Biliousness - Appearance of the tongue - Aphtha - "Throat-ail" - Sour taste in the mouth - Constipation - Diarrhea Backache - The stools - The urine-Dryness of the skin - Skin eruptions - Cold feet, etc.-HeadacheMigraine, or nervous headache - Nervous apoplexy Disturbance of the circulation-Nocturnal asthma of indigestion - "Stomach cough" - Nervous symptoms - Nervousness - Disturbances of sight, hearing, etc. Nervous diseases - Unusual drowsiness - Sleeplessness Mental disorders - Dilatation of the stomach - Prolapse of the stomach.

TREATMENT OF DYSPEPSIA

Diet and regimen - Dietetic rules - Diet tables - Diet List No. 1, ascptic dietary - Diet List No. 2, dry dietary - Diet List No. 3, nitrogenous dietary - Diet List No. 4, farinaceous preparations - Diet List No. 5, milk - Diet List No. 6, eggs and milk - Diet List No. 7, easily disintegrated foods - Diet List No. 8, anti-fat dietary - Diet List No. 9, fat and blood dietary - Diet List No. 10, fruits and nuts - Diet List No. 11, liquid dietary - Diet List No. 12, fever diet-Diet List No. 13, unfermented breads Diet List No. 14, for a bottle-fed infant under six months of age-Diet List No. 15, for a teething infant 
- Diet List No. 16, for a child from one to five years of age - Diet List No. 17, eating for strength - Diet List No. 18, diet for adults - Diet List No. 19, for rheumatism and gout - Diet List No. 20, for gastritis - Diet List No. 21 , for constipation - Diet List No. 22, articles difficult of digestion-Foods not easy of digestion - Diet List No. 23, foods which promote fermentation and decomposition in the stomach-Diet List No. 24, nutritive enemata - Diet List No. 25, health fools - ZwiebackGranose - Granola - Gluten biscuit - Caramel-cereal Bromose-Nut meal and nut butter-Gofio-Kumy zoon - Flesh food - Antisepsis of the mouth - Simple dyspepsia - Acid dyspepsia-Bilious or foul dyspepsia, or septic indigestion - Painful dyspepsia - Gastric neurasthenia, or nervous dyspepsia - Hypopepsia and apepsia - What about pepsin? - Hyperpepsia - Catarrll of the stomach - Stareh indigestion - Intestinal indigestion-Torpid liver - Infantile dyspepsia- In important caution - Chronic catarrh of the stomachUlcer of the stomach-Cancer of the stomach-Constipation.

\section{REMEDIES FOR THE HOME TREATMENT OF DYS-} PEPSIA

The sponge or hand bath - The full bath - The salt glow - The shower bath - The rubbing wet-sheet The wet-sheet pack - The trunk pack-The hot and cold trunk pack-The wet girdle--FomentationMustard or turpentine fomentation - Dry heat - The foot bath - The sit\% or hip bath - The sun bath - The vapor bath - The hot-air bath - Hot and cold to the spine-The hot and cold pour, or pail douche-The hot and cold full bath - The enema - The coloclys. ter-The cold and the graduated enema-The laxative enema-The oil enema-Oil rubbing - Massage, kneading - Abdominal massage - Massage of the bowels-Massage of the stomach-Replacement of the viscera - Inspiratory lifting of the abdominal contents - Hacking - Beating - General massage - Exercise-Exercises to develop the abdominal muscles Breathing exercises - Chest lifting - Manual Swedish movements - For general debility and anemia - For dilatation of the stomach - For prolapse of the stomach, bowels, colon, kidneys, and other abdominal organs - 
For dyspepsia with dilatation of the stomach-For constipation - The dry abdominal bandage - The abdominal supporter - Lavage - Hot and cold lavage of the stomach - Charcoal - Charcoal tablets - Antiseptic charcoal tablets - Lactic acid - Hydrochloric acid Laxatives - Cascara sagrada - Aqua salina - Seltzer Sulphur - Antiseptic dentifrice - Cinnamon solution - The Perfection Vaporizer-Benzoin solution Guaiac solution - Subcarbonate of bismuth - Subgallate of bismuth - Bicarbonate of soda-Local applications of faradic electricity-General app.ications of faradic electricity - The high tension sinusoidal current - The low tension sinusoidal current-Galvanization of the spiue and abdomen-Galvanization of the sympathetic nerve - The rest-cure - General nutritive treatment-General tonic treatment-Mechanical Swedish movements - The vibrating chair - Mechanical kneading - Trunk roiling - Pelvis tilting - Mechanical respiration - Cannon-ball massage - Water-drinking - The hunger-cure - Healthful dress - Sleeping - Traveling - Mental and moral treatment.

QUACKS AND NOSTRUMS

Stoughton Bitter's - Brown's Iron Bitters - Hop Bitters - Hostetter's Bitters-German Bitters - Stomach Bitters - French Absinthe-Vinegar Bitters-Lee's Antibilious Pills-Tropic Fruit Laxative-Carter's Little Liver Pills - Simon's Li ver Regulator - Radway's Regulating Pills-Eno's Fruit Salt-Hamburg Tea-Garfield Tea - Holloway's Pills - Hamburg Drops - R. T. Pierce's Pleasant Purgative Pellets - 1)r. Hall's so-called "Secret."

\section{GRAPHIC METHOD}

Explanation of the Author's Graphic Mode of Representing the Results of Stomach Work, obtained from the Examination of the Stomach Fluid by the System of Investigation Employed in the Hygienic and Physiological Research Laboratories of the Sanitarium at Battle Creek, Mich., U. S. A.- The coefficients of digestive work. 


\section{LIST OF ILLUSTRATIONS.}

Fig. 1.-The Salivary Glayds . . . . . . 22

Fig. 2.-The Temporarx Teeti . . . . . . 23

Fig. 3.-The Permanext Teeth . . . . . 24

Frg. 4.-The Stomach . . . . . . . 25

Fig. 5.-Peptic Gland . . . . . . . . . $\quad$. 26

Fig. 6.-The Almentary Chisal . . . . . 2

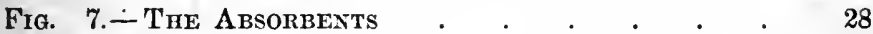

Fig. 8.-A. Pancreas; B. Duodexcy ; C. Spleex - 30

Fig. 9.-Starch Grantles . . . . . . 32

Fig. 10.-Tine Mesenteric Glands axd Lacteals . . 50

Fig. 11.- Diagram of the Circulatory Systens . 51

Fig. 12.- Human Teeth Covered with Tartar . . . 64

Fig. 13.- Human Teetil Injured by Tartar . . 64

Fig. 14.-Teeth of Healthy Cow . . . . . 101

Frg. 15. - Teeti of Cow Fed on Distilleny Slops • 102

Fig. 16. - Vinegar Eels . . . . . . . . . 113

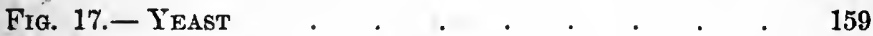

Fig. 18. - Uric Acid Crystals . . . . . . . . 189

Frg. 19.-Shower Bath . . . . . . . : 291

Fig. 20.- Simple Shower Bath . . . . . . 292

Fig. 21.-Rubbing Wet-Sheet . . . 293

Frg. 22.-Wet-Sheet PACk . . . . . . . . 294

Fig. 23.-Fomentation . . . . . . . . - 295

Fig. 24.-Hot Water-Bag . . . . . . . . 296

FiG. 25.-SPINE BAG . . . . . . . . 296

Fig. 20.-Foot BAth . . . . , . . . 297

Fig. 27.-The Sitz Batr Túb . . . . . . 298 
Fig. 28. - VAPOR BATH . . . •. . . " . . 299

Fig. 29.-SyPhoN SrRinge . . `. . . . 301

Fig. 30.-Fountain Syringe . . . . . . . 302

Fig. 31.- Water Bottle and Syringe Combined . 303

Fig. 32.-Arim Flexion . . . . . . . 308

Fig. 33.- Amm Extensiox . . . . . . . 309

Fig. 34.-Exercise to Strengthex Trunk Muscles _ . 312

Fig. 35.-,Lying, Kree Bexding . . . . . . 314

Fig. 36.-Chest Lifting . . . . . . . 315

Fig. 3\%-Sacrum Beating . . . . . . 316

Fig. 38. - Exercise for Abdominal Muscles . . . 318

Fig. 39.-Standing, Sidewats Bexdixg . . . . 310

Fig. 40,- Natural Abdominal Supporter . : . 320

Fig. 41.-Stomacn-Tube . . . . . . . . - 321

Fig. 42.-Penfection Vaporizer . . . . . 325

Fia. 43.- Vaporizer in Use . . . . . . . 325

Fig. 44.-Sinusoidal Electrical Apparatus . . . 328 


\section{LIST OF PLATES.}

Plate I. - The Internal Organs (colored)

PAGE.

Frontispiece.

\section{Plate II.-Micro-Organisms . . . . . . 128}

Fig. 1.- Milcrococcl arranged in chains (streptococci).

Fig. 2.-Micrococcl in pair. (diplococei).

Fig. 3.- Micrococci in a mass or swarm (zoogloa).

Fig. 4.- Micrococci arranged in groups of four, found in the sputa of consumptive patients.

Fig. 5.-Micrococci of pneumonla.

Fig. 6.-Bacteria termo, found in decomposing matter.

Fig. 7.-Bacteriae termo, magnified 4000 diameters.

Fig. 8.-Bacillus subtilis, hay bacillus found on manure or in a decoction of hay.

Fig. 9.-Bacteria lineola, germs from stagnant water ; sometimes seen in slimy masses on rotten potatoes ; also found in well-water.

Fig. 10.-Bacillus of typhoid fever.

Fig. 11.-Bacillus of malaria, found in the blood in cases of malarial fever.

Fig 12.-Bacillus of tuberculosis.

Fig. 13.-Bacillus of leprosy.

Fig. 14. - Spirilla undula, found actively moving in decomposing infusions.

Fig. 15.-Cholera germs.

Fig. 16. - Spirillum volutans, a germ with flagelia, found in marsh water.

Fig. 17.-Vibrio regula, germs found in decomposing vegetable matter.

Fig. 18.-Cladothrix dichotoma, common germ found in water containing decomposing animal or vegetable matter.

Fig. 19.-Rhabdomonas rosea, germs which form the red-colored scum on ponds.

Fig. 20.-A germ similar to the precedlng, of a pale-red color, found in stagnant water.

Fig. 21.- Germs found in stagnant water and in drain pipes.

Fig. 22.-Bacterium aeeti, the germ which produces vinegar.

Fig. 23.- Germs of cholera.

Fig. 24.-A germ which causes fermentation.

Flg. 25.-Germs of anthrax in blood from spleen of mouse.

Fig. 26.- Germs of anthrax grown on a potato.

Fig. 27.-Germs of anthrax at a different stage of growth.

Fig. 28. - Foul water germ In different stages of growth.

Plate III.-Animalcules and Infusoria in Water . 129

Plate IV.-Displacements of the Stomach . . . 135

Fig. 1.- Visceral displacement.

Fig. 2.-Displaced viscera.

Fig. 3. - Results of corset constriction (woman of thirty).

Fig 4.-Displacements of spleen and other viscera.

Fig. 5.-Woman who had worn a "health corset."

Fig. 6.-Bad standing corrected (man). 
Fig. 7.- Bad standing corrected (woman).

Fig. 8.- Standing on one foot corrected.

Fig. 9.- Results of corset constriction.

Fig. 10.- Displacement of spleen and other viscera.

Fig. 11.- Woman who had worn a "bealth corset."

Plate V.-Germs (colored) . : . . . . . 18

Fig. 1.- Mouth germs found upon the surface of a coated tongue, greatly magnified.

Fig. 2.- Germs which cause decay of the teeth.

Fig. 3.-Germs found in the stomach fluid after a test meal, greatly magnified.

Plate Vi.- Movements of Massage

Fig. 1.-Superficial kneading.

Fig. 2.-Deep kneading.

Fig. 3.- Massage of the abdomen.

Fig. 4.- Massage of the bowels.

Fig. 5.- Massage of the stomach.

Fig. 6.- Replacing of the viscera.

Fig. 7.-Inspiratory lifting of the viscera.

Fig. 8.- Hacking.

Fig. 9.-Beating.

Fig. 10.- Exercise to strengthen the abdominal organs.

Fig. 11.- Knees separating with resistance.

Fig. 12.-Fult breathing.

Plate VII.- Nass.lge

Fig. 1.- Trunk rolling.

Fig. 2.-Vibrating chair.

Fig. 3.-Pelvis tilting.

Plate Vill. - Massage

Fig. 1.- Mechanical kneading.

Fig. 2.-Cannon-ball massage.

Fig. 3.-Mechanical respiration.

Plate IX. - Healthful and Artistic Dress

Fig. 1.- Variété Costume.

Fig. 2.-Freedom Waist with skirt.

Fig. 3.- Norfolk Jacket with skirt of Business Suit.

Fig. 4.-Woman's Practical Business Costume.

Plate X. - Healthful Dress

Figs. 5 and 6.-Divided Skirt with circular yoke. Knickerbockers.

Fig. 7.- Woman's Practical Business Costume, with skirt opened in back to show divide.

Figs. 8 and 9.-Skirt waist.

Fig. 10.-Skirt with circular yoke.

Figs. 11 and 12.-Union suit.

Plate XI.-Graphic Method (colored) . . . . 352 


\section{THE STOMACH :}

\section{Its Disorders and How to CuRE Them.}

Some years ago, while calling upon a professional friend in London, a leading English practitioner, he remarked, "I suppose that in America you have chiefly to deal with dyspeptics." In reply, I could not but acknowledge that Americans enjoyed the unenviable, but nevertheless deserved, reputation of being a nation of dyspeptics; but added that the treatment of invalids in America was by no means so monotonous as might seem to be the case, for the reason that the general physical deterioration and vital derangements occasioned by dyspepsia give rise to a vast number of varied and complicated ailments which tax to the utmost the skill and ingenuity of the practitioner.

Indigestion, however, is by no mẹans confined to America. The special manifestations of impaired digestion which are experienced in the stomach itself, and which most readily attract attention, are perhaps more common in the United States than elsewhere, being a natural result of the hasty and disorderly habits of eating so usual among us. In England, Australia, New Zealand, and other English colonies, while there is less complaint of indigestion, there is, nevertheless, evidence of the same prevalence of derange- 
ments in the digestive and nutritive processes, as is shown by the frequency with which rheumatism, gout, kidney and bladder diseases, and various nervons affections are encountered.

Modern researches into the relations of varions maladies have shown that not only Bright's disease and the other complaints mentioned, but also pulmonary consumption, jaundice, many affections of the liver, typhoid fever, tapeworm, malarial disease, organic diseases of the spine and brain, and even insanity, are, in a large proportion of cases, due either directly or indirectly to the vital disturbances arising from a morbid condrtion of the stomach.

Dyspepsia is unquestionably the foundation of the greater share of all chronic maladies. When in a healtliy state, the body is able to defend itself, not only against germs, but against all other ordinary causes of disease; but the general vital deterioration resulting from indigestion weakens the defenses of the body and its vital resistance, and renders it a prey to every malady.

The function of a tissue or an organ depends upon its structure; and the structure of every cell and fiber of the body is dependent upon the quality and quantity of the material absorbed from the alimentary canal. A deficient supply of food weakens the structure and lessens, the energy of the organ. An excess of food overwhelms the tissues with imperfectly oxidized and toxic substances, whereby their structure is deteriorated, and their functions perverted or retarded. Food containing toxic substances produces in the body general or specific toxic effects. The same thing results from 
the development of toxic substances in the alimentary canal from the fermentation or putrefaction of food in the stomach and intestines.

Pasteur, David, and others have shown that the mouth, the stomach, and the intestines are continually inhabited by a vast number of microbes capable of producing various acids, poisonous ptomains and toxins, varying in their physical and physiological properties, some of them being capable of producing powerfully poisonous effects. These poisons exist in greater or less quantity; but, owing to the poison-destroying power of the liver and the eliminative function of the kidneys, the amount at any time circulating in the tissues of a healthy person is not sufficient to produce any more deleterious effect than that gradual deterioration of the organism by which the tissue-modifications characteristic of old age are brought about. When, however, diseased conditions of the stomach or intestines exist, these poisons may be increased to an enormous degree; for example, Bouchard has shown that if food is retained in the stomach more than five hours, the changes which take place are fermentative and putrefactive, rather than digestive. This explains the relation demonstrated by Bouchard and others between dilatation of the stomach and chronic rheumatism, and the lowered vital resistance which, by dyspepsia, prepares the way for tubercular disease of the lungs.

That something is wrong with the modern stomach is evident from the great number of remedies advertised in the newspapers, and displayed upon every druggist's shelves, as capable of aiding a weakened stomach. Pepsins, peptones, peptonoids, and various digestive 
ferments are manufactured by the ton. Notwithstanding all these helps, however, the average stomach in civilized lands grows weaker year by year, and disorders growing out of indigestion multiply with alarming rapidity. Many persons suffer from disorders of digestion without being aware of the fact. Headache, backache, bladder and kidney disorder's, sleeplessness, depression of spirits, weakness, lack of energy, coldness of the extremities, nervous sensations of various sorts, even hysteria, epilepsy, and insanity, may exist as the result of indigestion without any suffering in the stomach, or anything to suggest to the patient himself or to the untrained observer, any disorder of digestion. In every case of chronic disease, the stomach should be carefully investigated. A coated tongue is always indicative of a disordered stomach. The samo is true of numerous other symptoms from which many people suffer, such as a bad taste in the mouth, dulness or headache after eating, nervous headiche, etc.

Indeed, dyspepsia is a malady which demands the most serious attention. This is especially apparent when we recognize the fact that, of all chronic ailments, it is preeminent as being preventable by the employment of proper means. No disease is more dependent upon conditions which the individual himself can control than is this; neither is there any malady which more readily yields to the application of appropriate remedies when the necessary conditions are supplied. I have no hesitancy in asserting that every case of purely functional disease of the stomach may be cured, provided the requisite conditions can be obtained. 


\section{THE ORGANS OF DIGESTION.}

The Healthy Stomach. - The conditions of a diseased stomach cannot be understood without a clear knowledge of the healthy stomach and its functions. Digestion is the process by which the food is dissolved and rendered capable of absorption into the body. All animals digest, and even plants digest by means of their leares. The following brief description of the digestive process is largely taken from a text-book of physiology and hygiene by the author: ${ }^{1}-$

The Alimentary Canal. - The process of digestion is chiefly performed in a long tortnous tube called the alimentary canl, which is from twenty-fire to thirty feet in length, and reaches from the mouth to the lower part of the trunk.

The digestive organs consist of this canal and other organs closely connected with it, including various glands. The latter produce peculiar fluids, by which the food is changed in several very remarkable ways, as we shall presently learn. The several organs which take part in the process of digestion may be named as follows :-

The mouth, tongue, teeth, salivary glands, esophagus, stomach, small intestines, colon, liver, and pancreas. (The relative position of the liver, stomach, and intestines is, well shown in Plate I.)

1 "Second Book in Physiology and Hygiene," by J. H. Kellogg, M. D., published by the American Booik Company. The work may be obtained from the Modern Medicine Publishing Company, Battle Creei, Mich. 
The Mouth.- The cavity of the mouth is the expanded upper portion of the alimentary canal. It contains the teeth and the tongue, and receives small ducts, or canals, from the three pairs of salivary glands closely connected with it. These glands produce a fluid known as saliva. At the back part of the mouth are found, one on each side of the tonsils, glands which

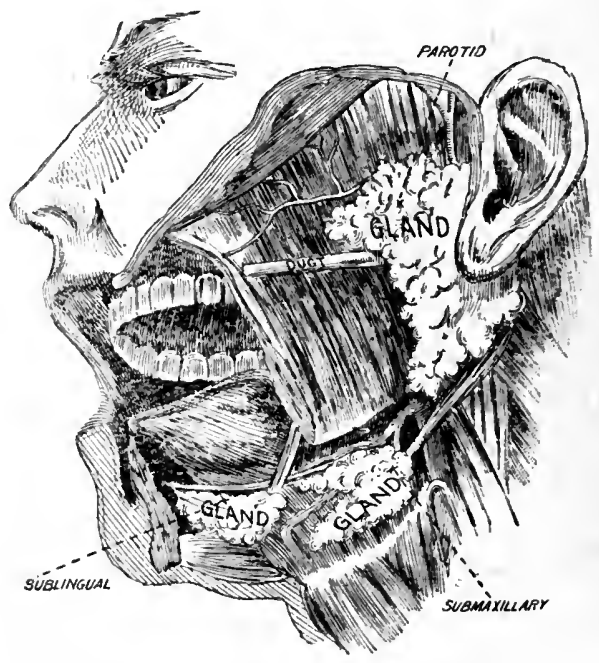

Fig. 1.-The Salivary Glands.

help to form the saliva. The saliva contains an active principle known as ptyalin. (Fig. 1.)

The Teeth. - A tooth presents three parts for examination: the portion which is seen above the gum, called the crown; the portion which is embedded in the jaw, called the root; and the narrowed portion which joins these two parts, called the neck. The interior of the tooth is filled with a fleshy substance, 
the pulp, containing the blood-vessels and the nerves which nourish the tooth and give it sensibility. The body, of the tooth is made up of a tissue resembling bone. The portion which projects from the gum is covered by a very hard, smooth substance, called enamel, which is so brittle as to be easily broken by violent contact with hard substances.

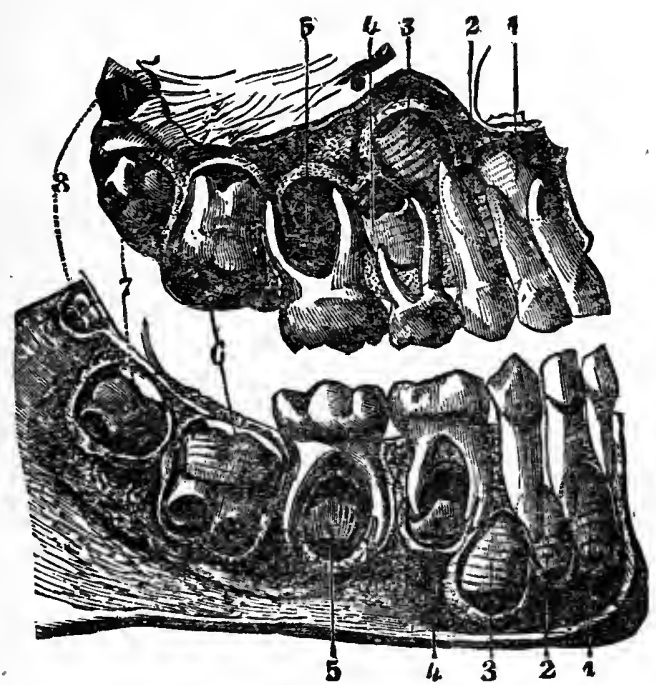

Fig. 2.-The Temporary TeEth.

Temporary Teeth. - The teeth which appear in infancy and early childhood are called temporary, or milk, teeth. These teeth usually make their appearance between the ages of seven months and two years. They are twenty in number, and consist, in each jaw, of four front teeth, or incisors, two cuspids, one on each side of the incisors, and four molars, or double teeth, two on each side. (Fig. 2.) 
Permanent Teeth. - At the age of six or seven years the temporary teeth begin to give place to the permanent set, which, when complete, at the age of seventeen to twenty, numbers thirty-two, each jaw con-

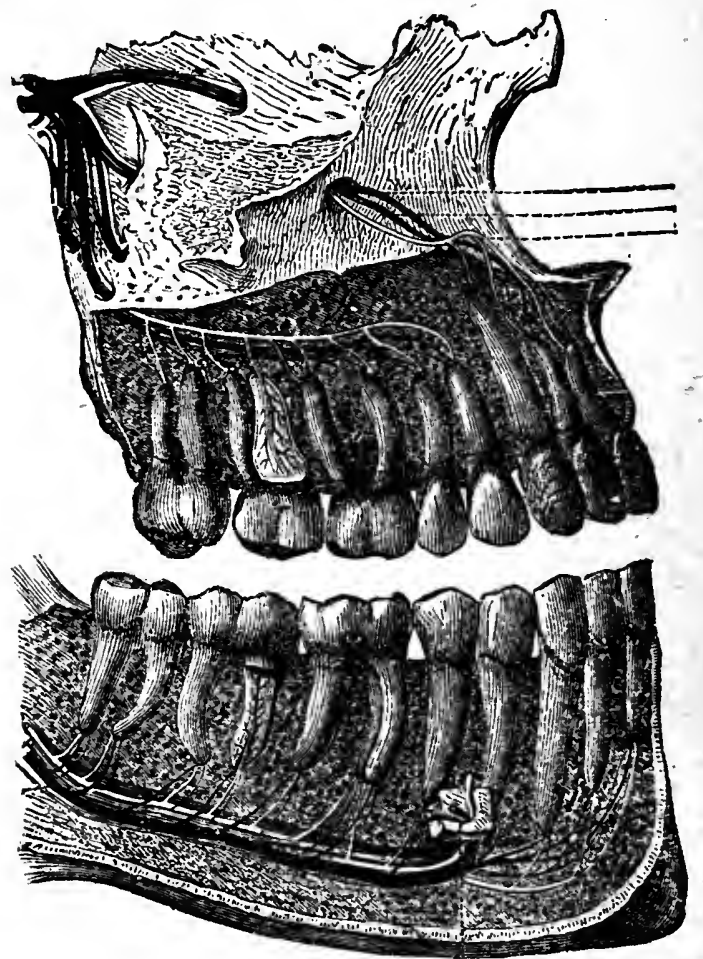

Fig. 3.-The Permanent Teeth.

taining, in addition to those of the temporary set, four small double teeth, or bicuspids, and two additional molars, the so-called wisdom teeth. The last of the temporary teeth should give place to the permanent ones not later than the twelfth year. (Fig. 3.) 
The Esophagus. - In the act of swallowing, the food passes from the mouth downward through a narrow passage about nine inches in length, commonly called the esophagus, or meat-pipe. The walls of this canal are made up in part of muscles, by the aid of which the food is carried through it. When empty, its sides lie in contact with each other. At its lower end a circular muscle guards the opening into the stomach.

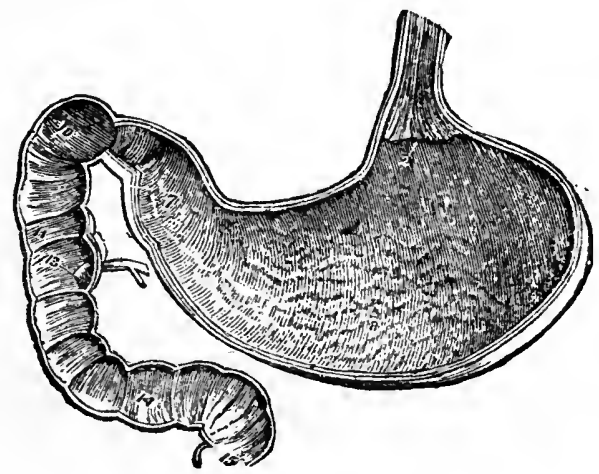

Fig. 4.- The Stomach.

The Stomach.- This is a dilated portion of the alimentary canal, somewhat pear-shaped in form, and capable of holding from one to two quarts. The walls of the stomach, like those of the entire digestive canal, are made up largely of thin layers of muscle, by means of which it is able to change its size and shape, and so to act upon the food as to produce a sort of churning action. (Fig. 4.)

The Peptic Glands.-The lining membrane of the stomach, when examined with a microscope, is found to present multitudes of minute openings, 
each of which is found, on further examination, to communicate with a narrow tube which is embedded in the walls of the stomach. This little pocket is lined with minute living cells, which, during the digestion of a meal, are engaged in making a most important digestive fluid, the gastric juice. (Fig. 5.)

The Gastric Juice.- This fluid is intensely acid. The acid of the gastric juice is hydrochloric acid, probably not a purely chemical acid, but an organic

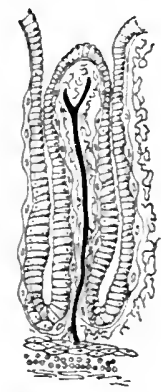
hydrochloric acid. There is also present a small amount of lactic acid.

The gastric juice contains two digestive principles, pepsin and rennin.

The Pylorus.- Each end of the stomach is guarded by a circular muscle. That at the lower, or right, end is known as the pylorus. By means of these muscles, the openings of the stomach are kept Fig. 5.-PEPTIC tightly closed while the process of digestGLAND. ion is going on.

The Small Intestine. - This portion of the alimentary canal is about twenty feet in length, reaching from the stomach to the large intestine, which it joins at the lower right portion of the cavity of the abdomen. The walls of the intestine are muscular, like those of the stomach. Its mucous lining contains many glands, which produce an alkaline digestive fluid known as intestinal juice; and it is also covered with giant cells, which defend the body against germs. During digestion, the muscular walls of the intestines are in constant motion. (Fig. 6.) 
ORGANS OF DIGESTION.

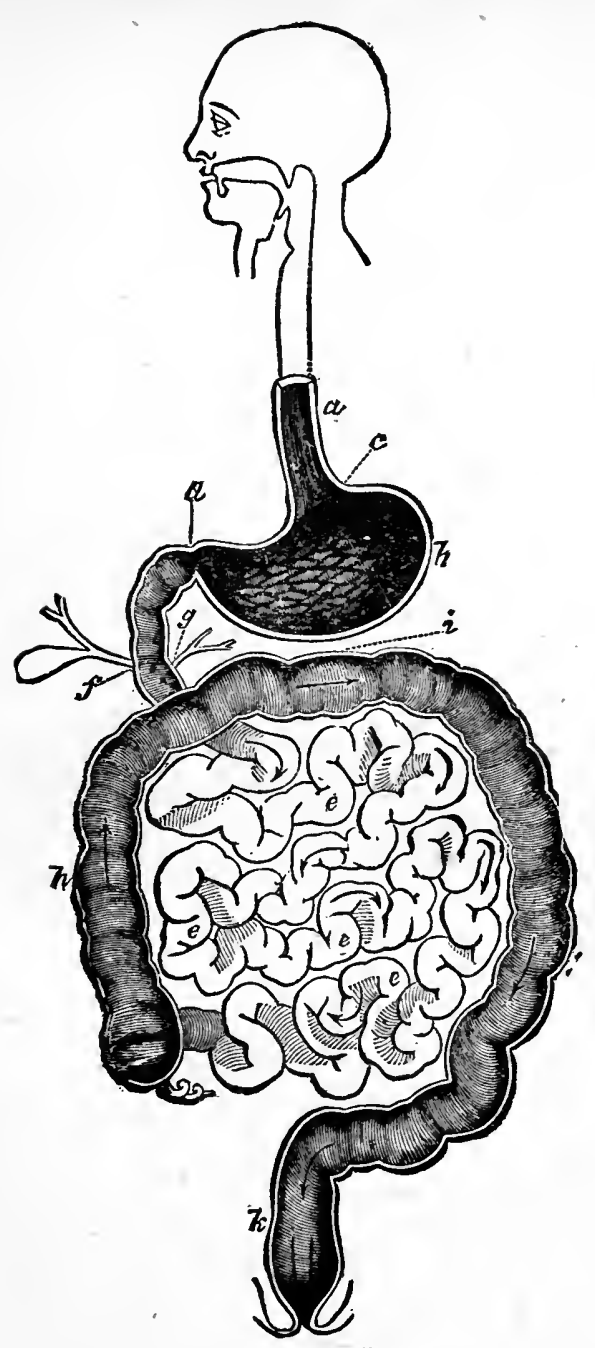

Fig. 6.-The Alimentary Caxal. 
The Intestinal Juice.- This, the most complicated of all the digestive juices, is produced by the action of all the numerous and varied glands found in the mucous membrane of the intestines. Being composed thus of the secretions of a number of different glands, it is of a compound character, which especially fits it for its raried functions.

The Absorbents. - The mucous membrane of

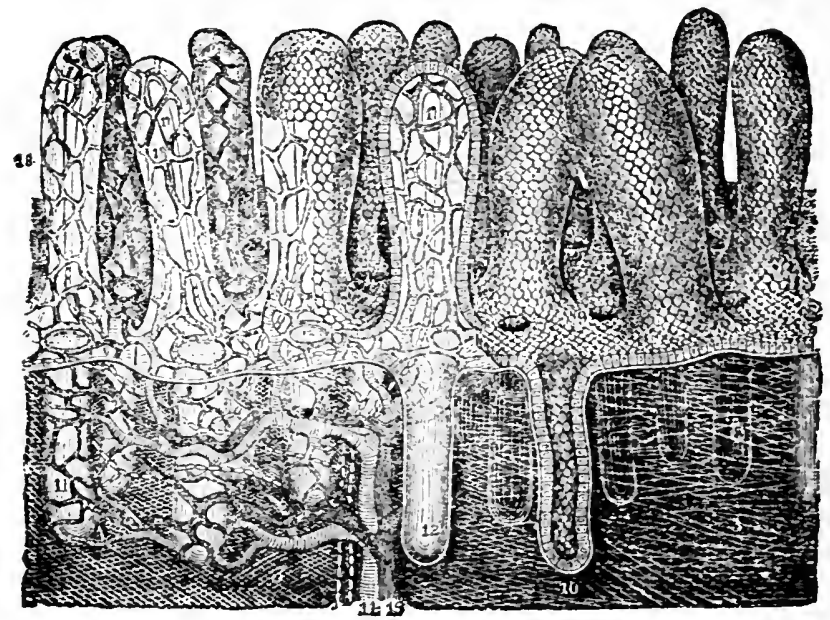

Fig. 7.-THE A BSORBENTs.

the small intestine contains numerous folds, and also peculiar structures for aiding absorption, known as the villi. The villi are hair-like projections of mucous membrane, which hang out into the cavity of the intestine. Each villus contains blood-ressels and lymph-channels. (Fig. 7.)

The Large Intestine, or Colon.- This portion of the alimentary canal is about five feet in length, and is much larger in diameter than the small intes- 
tine. It begins at the lower right side of the abdominal cavity, where it is joined by the small intestine. From this point the colon passes upward to the lower border of the ribs, crosses over to the left side, then passes down to the lower left portion of the abdomen, and thence backward, finally terminating in the rectum.

The Liver. - Lying chiefly at the right side, just above the lower border of the ribs, and partly covering the stomach, is the largest gland in the body, the liver. At the under side of the liver is a sac, or pouch, known as the gall-bladder, in which is stored up the digestive fluid formed by the liver, called the bile. A short canal, or duct, connects both the gall-bladder and the liver with the small intestine at a point a few inches below the stomach.

The Bile.-Human bile is alkaline, somewhat viscid, and of a golden-brown color when fresh. When vomited, bile is often green or yellow, as the result of changes produced in the stomach by contact with the acid gastric juice. The bile of the ox is naturally of a greenish color.

A remarkable fact in relation to the structure of the liver is that it is supplied with two sets of blood-vessels. The extra system of vessels brings to the liver the blood from the stomach, intestines, and other digestive organs, and constitutes the portal circulation.

The Pancreas.-Just behind the stomach is a long, curiously shaped gland, known as the pancreas, which secretes the pancreatic juice.

The Pancreatic Juice.-This is a fluid much like saliva. It is produced during the digestion of a meal, and is poured into the small intestine through a 
duct which joins that from the liver, and with it opens into the intestinal canal four or five inches below the stomach. (Fig. 8.)

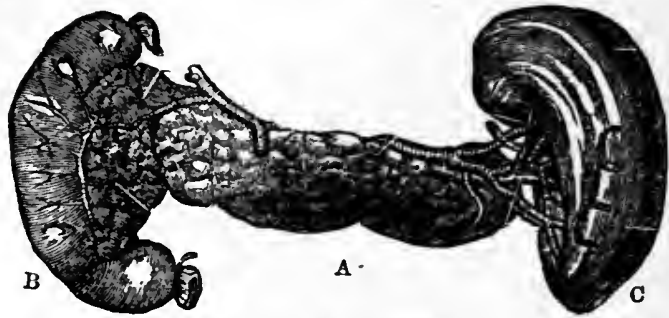

Frg. 8.-A. Paxcreas ; B. Deodexum ; C. Spleen.

The pancreatic juice contains four active prineiples : amylopsin, trypsin, steapsin, and a milk-curdling ferment.

In order readily to understand the relation of the digestive organs and their several digestive fluids to foods, it is necessary to briefly consider the question, What are foods? 


\section{FOODS.}

THE body is constantly sustaining losses in consequence of the vital work performed by its various organs. Foods are substances which, when introduced into the body, make good its natural wastes and losses, and furnish proper material for the repair of its tissues, or for carrying on its vital processes. These requirements are met by organized matter, water, and oxygen, or what may be termed solid, liquid, and gaseous foods. Of these, the first only is commonly known as food. Liquid foods are called drinks, all of them having water for their essential element. Our attention will here be confined to the consideration of organized foods.

A poison is the opposite of a food. It not only does not repair wastes and losses, but interferes with the vital processes, disturbing them in such a way as to occasion sickness and death.

Animal and Vegetable Foods.-Man employs both animal and vegetable substanees as foods. Some nations, particularly the English and the American, use a large proportion of flesh, and some barbarous tribes live almost wholly upon it ; but the larger portion of the human race live chiefly upon vegetable foods. Many millions of human beings in India and other parts of Asia never taste flesh food, considering it a sin to do so.

Plants the only Food.Producers.-Plants alone possess the power to construct living substances 
out of the elements of the earth and the air. Animals are able to subsist upon organized substances only, so that a lion, in dining upon an antelope, is only eating at second hand the grass and herbs which the latter has eaten ; and a man, in eating roast beef, is taking at second hand the corn upon which the ox was fed.

Food. Elements. - When a chemist examines a loaf of bread or a piece of meat, he finds it to be made up of various substances quite unlike in nature. These

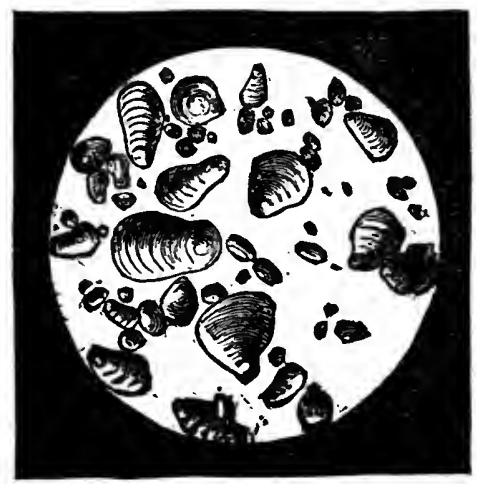

Fig. 9.-Starch Grantles. food elements each possess peculiar properties, and are destined for different uses in the body when taken as food.

Classification of F o od Elements.The various substances found in foods may be included in six classes : 1. Starch ; 2. Sugar ; 3. Albumen (all albuminous substances); 4. Fats; 5. Salts ; 6. Indigestible elements.

Starch.-- This element is found only in regetable foods. In a law state, starch is found in small particles, or granules, each enclosed in a woody envelope. Starch is the most abundant of all the food elements. (Fig. 9.)

Sugar.-Sugar is very unlike starch in its general properties, although closely related to it. In the mysterious chemistry of plant life, the insoluble, tasteless starch is converted into this sweet and extremely 
soluble substance. Several different kinds, of sugar occur in nature, the most important of which are canesugar, grape-sugar, and milk-sugar.

Cane-sugar is the sweetest of all the sugars, and is that commonly used as food. It is obtained from the sugar-cane, the sorghum plant, the beet root, and the maple-tree. Grape-sugar is found in most fruits and in honey. Milk-sugar gives to milk its sweetness. A sugar resembling grape-sugar, called glucose, is very extensively mannfactured chemically, by boiling the starch of corn or potatoes with sulphuric acid. Glucose cannot be considered a perfect substitute for natural sugar.

Albumen.-- The white of an egg is almost pure albumen. All true foods contain elements which in many respects resemble albumen, and serve the same purposes ill the body, and so are termed albuminous elements. For convenience, we shall apply the term albumen to any or all of them. The lean portion of flesh and the casein of milk are forms of animal albumen. All vegetable foods also contain albumen. Cascin, for example, is found in peas and beans, as well as in milk. One of the most important of all the albumens is gluten, which is found in wheat, rye, and barley.

Fats.-Oil, or fat, is found in both animal and vegetable foods. The principal animal fats used as food are butter, lard, suet, and tallow. Vegetable oils are chiefly derived from oily fruits, as the olive, from nuts, and from various seeds. A large quantity of fat is found in corn and oats.

Salts. - When a portion of animal or vegetable 
food is burned, there is left a residue of ashes, made up of inorganic, or mineral, elements. These are the so-called salts of the food. They do not exist in the food in the form in which they are found in its ashes, but in an organized form. Grains constitute the most important source of salts. Wheat, oats, barley, corn, and rye contain an abundant supply of this element, as do the potato and most other vegetables. The salts also exist in milk in good proportion.

Indigestible Elements. - All vegetable foods contain more or less of a woody substance, called cellulose. The bran of wheat belongs to this class of elements. Cellulose is not to any extent digestible, but it serves an important purpose in giving bulk to the food. The connective-tissue elements of flesh foods - the ligaments, tendons, etc. - are hard to digest, and afford little or no nourishment.

In addition to the several elements mentioned, all food substances contain certain flavoring matters.

Condiments. - A condiment is an article which possesses little or no food value, but is added to food for the purpose of imparting to it a characteristic flavor. The condiments most commonly used in this country are mustard, pepper, ginger, spices, pepper-sauce, Worcestershire and other hot sances, and vinegar. All condiments possess irritating or stimulating qualities. They stimulate the appetite, and act as whips to the stomach and other digestive organs, and are for this reason injurious.

Food Substances. - None of the several food elements which we have been considering are, in any 
proper sense, to be regarded as food. An animal fed exclusively upon any one of them soon acquires such a disgust for its food that it will refuse to taste it, even though starving, and sooner or later dies. Gluten is the only exception to this rule. A true food contains various elements, which are combined in varying proportions in different foods. Let us now briefly notice some of the leading food substances.

Foods of Animal Origin. - Chief among animal foods is mill, the natural diet of most young animals. Milk contains the different elements of nutrition in proper proportion, and will sustain life for an indefinite period. The chief albuminous element of milk is casein. The white color of milk is due to the fact that it contains a considerable amount of fat, or oil, in a state of emulsion, or division into minute drops. By churning, the little drops are made to unite, producing butter. The ease with which it is digested renders milk a most suitable food for the young. It is, indeed, with rare exceptions, a most wholesome food for persons of all ages.

Cheese made from milk by adding rennet, which separates the casein and fat from the whey, undergoes partial decomposition in the process of "curing," and is on this account much less wholesome than fresh milk. It is difficult to digest, and likely to interfere with the digestion of other foods. Sometimes a peculiar fermentation takes place in cheese, which produces a very poisonous substance, known as tyrotoxicon. Very serious and sometimes fatal illness often results from the use of such cheese. This poison is destroyed 
by heat. On this account cheese is rendered less dangerous by toasting, while at the same time it is made more digestible.

Eggs:- An egg contains within itself every element needed for the support of the body, and has the advantage, when properly cooked, of being one of the most easily digested of foods, and one of very high nutritive value. When stale, however, eggs are exceedingly deleterious, and wholly unfit for food. An egg nsually keeps perfectly fresh not more than three or four days, when exposed to the ordmary summer temperature. Eggs laid by fowls fed upon decaying meat and other muwholesome food, are unfit to eat.

Flesh.- The flesh of the ox, sheep, and hog is more largely used as food in this and most other civilized countries than any other kinds of flesh food. Mutton is not so well relished as beef by some, but it is nearly as nourishing, and equally as easy to digest. Pork contains much fat, and is difficult to digest, besides being likely to be diseased, and must be regarded as an inferior food. The Jews in ancient times were forbidden to eat the flesh of the hog, and they still abstain from the use of pork. The flesh of deer and other wild game, while usually less tender than that of stall-fattened animals, is more wholesome, if eaten when fresh, on acconnt of the healthier conditions of life which wild animals usually enjoy. Game is often allowed to become almost putrid before it is eaten; such flesh is exceedingly unwholesome. Veal, like the flesh of all very young animals, is difficult to digest, and cannot be recommended as food.

Fish and Fowl.- The nutritive value of fish 
and fowl is not quite equal to that of beef or mutton; but when properly cooked, they are relished by most persons, and possess considerable value as foods.

Shell-fish contain very little nutriment, although some of them, oysters in particular, are in very great favor as table delicacies. All shell-fish are scavengers, however, and are sometimes poisonous. Frogs, lobsters, shrimps, sea-crabs, etc., are by many considered delicate eating, but cannot be regarded as really firstclass foods. The oyster is easily digested, though it does not possess the power to digest itself, nor to aid digestion when eaten raw, as many persons suppose. The oyster is a scavenger in its habits; and when the beds in which oysters grow are located so as to be reached by the impure matters carried into the sea by the sewers of a large city, the oysters sometimes become diseased, and produce serious illness when eaten. - Typhoid fever has been traced to the use of oysters.

Salted and Smoked Meats.-Most kinds of flesh foods are preserved by salting. The process of salting hardens the tissues, thus rendering them difficult to digest. Smoked meats and fish are also hard to digest.

Vegetable Foods. - Vegetable foods are the original source of the nutritive elements contained in flesh foods ; hence we should expect them to furnish all the elements of nutrition, and in proper proportions. This is the case with the best vegetable foods. Vegetable foods are usually divided into three classes :-

Fruits, Grains, and Vegetables.-Fruits comprise fleshy seeds and seed bearing portions of plants, such as the apple, strawberry, and plum, each of which 
represents a different class of fruits. Melons and nuts are also fruits. Grains comprise those sceds used as foods which are produced by grass-like plants, as wheat, oats, rye, barley, corn, and rice. Allied to this class are the edible seeds of pod-bearing plants, the chief of which are peas, beans, and lentils. Grains are the most nourishing of all foods, and contain the elements of food in the best proportion. Fruits, grains, and milk constitute a perfect dietary, and one particularly suitable for young persons, and for students and other brain-workers.

Those parts of plants used as food, other than seeds or fruits, such as leaves, stems, roots, buds, and flowers, are called vegetables. The nutritive value of vegetables is much less than that of grains. The potato, one of the most valuable of all regetables, is three fourths water, and contains only about two per cent. of albuminous elements. The starch of vegetables is more difficult to digest than that of grains and fruits, and the large amount of woody matter contained in most vegetables adds to their indigestibility; so that they must be regarded, in general, as much inferior to fruits and grains as foods.

Peptogens. - Certain properties and elements of the food serve to stimulate the action of the glands by which the digestive fluids are produced. For example, the quality of dryness in the food powerfully excites the action of the saliva. In experiments conducted by the author in the Laboratory of Hygiene of the Battle Creek Sanitarium (Michigan, U. S. A.) it has been proved that dry food stimulates the secretion of saliva more than four times as much as moist food. 
Gluten, albumen, and other proteid substances stimulate the glands of the stomach to produce gastric juice. The same is true of dextrin. This fact is of considerable interest, as it emphasizes the importance of thorough mastication of the food. In this way the food has mixed with it an abundant quantity of saliva, by whose action upon the starch, the dextrin is produced.

The natural flavors of foods stimulate the activity of all the digestive glands; but condiments, such as mustard, pepper, pepper-sance, and other substances which burn and sting as they go down the throat, do not stimulate the secretion of normal digestive fluid. They simply provoke resistance and defense, causing an abundant flow of mucus wherewith to protect the mucous membrane from their harmful action.

The Natural Diet of Man.- It is probable that the diet of the human family at first consisted almost wholly of fruits, grains, milk, and a fow yegetables. History informs us that the clietary of the ancient Egyptians, Assyrians, and the early Greeks and Romans was of this simple character; and the same diet is still practically adhered to by fully two thirds of the inhabitants of the globe. In densely populated countries, such as Japan and China, the diet is necessarily almost exclusively vegetarian. The peasant class of France, Italy, and Spain, and other continental European countries, use flesh so sparingly that it may be said to be a luxury rather than a food with them. Human life and health can be well maintained upon vegetable food.

Uses of the Several Food Elements.-The various food elements serve different purposes in the 
body. Sugar, starch, and fat make adipose tissue, and in the form of fat enter into the composition of nearly all the tissues of the body. They are of important service to the body in the production of heat and force. The different forms of albumen nourish especially the brain, nerves, muscles, glands, and other highly actire tissues of the body. The salts are largely used in nourishing the bones. They are also required by the brain and the nerves, as well as by other tissnes. The indigestible elements gire necessary bulk to the food.

\section{DIGESTIBILITY OF VARIOUS FOODS.}

Hr. Min.

- Rice............... 100 Sigo ............... 1 4.5

- Tapioca................2 200

- Barley ..............2 200 Milk, boilesl............ 200

- Milk, raw...........2 21.5

- Tenison, broiled........ 1 3.5

- Turkey, roistci....... 2 30 Turkey, domestic, boiled 225

- Goose, roasted.......... 230 Lamb, broiled..........2 30 Eggs, hard boiled....... 330

- Eggs, soft boiled........ 300

- Eggs, fried............ $3: 30$ Eggs, raw..........2 200

- Eggs, whipped......... 130 Trout, boiled.......... 130

- Salmon, salted, boiled... 400

- Oysters, raw...........2 5.

- Oysters, stewed......... 330 Beef, lean, rare roasted... 300

- Beefsteak, broiled...... 300 Beef, lean, fried..........4 400

- Beef, salted, boiled..... 415

- Pork, roasted........... 5 15 Pork, salted, fried...... 4 1.5

- Mutton, roasted........ 315
Hr. Min.

Mutton, broiled........ \$ 00

Veal, broiled........... +00

- Veal, fried.............. 430

Fuwl, boiled........... 400

Duck, roasted............ 430

Butter, melted.......... ; 30

-Cheese............... 330

Soup, marrow-bone...... 4 15

Soup, bean........... ; 00

Soup, mutton .......... 3 30

Corn and beans, green ... 3 45

Chicken soup, boilea... 300

-Beans, pod, boiled...... 2 30

-Bread, wheaten ......... 330

- Bread, corn ........... 315

Apples, sour and mellow, raw ............... 200 Apples, sweet and mellow, raw ............ 130 Parsnips, boiled ........ 230

-Beets, boiled........... 345

Turnips, flat, boiled.... 330

Potatoes, Irish, boiled... 330

-Potatoes, Irish, baked... 230

Cabbage, raw.........2 30

Cabbage, boiled......... 430 
TABLE SHONING THE NUTRITIYE YALUR OF COMMON FOOD SUBSTANCES.

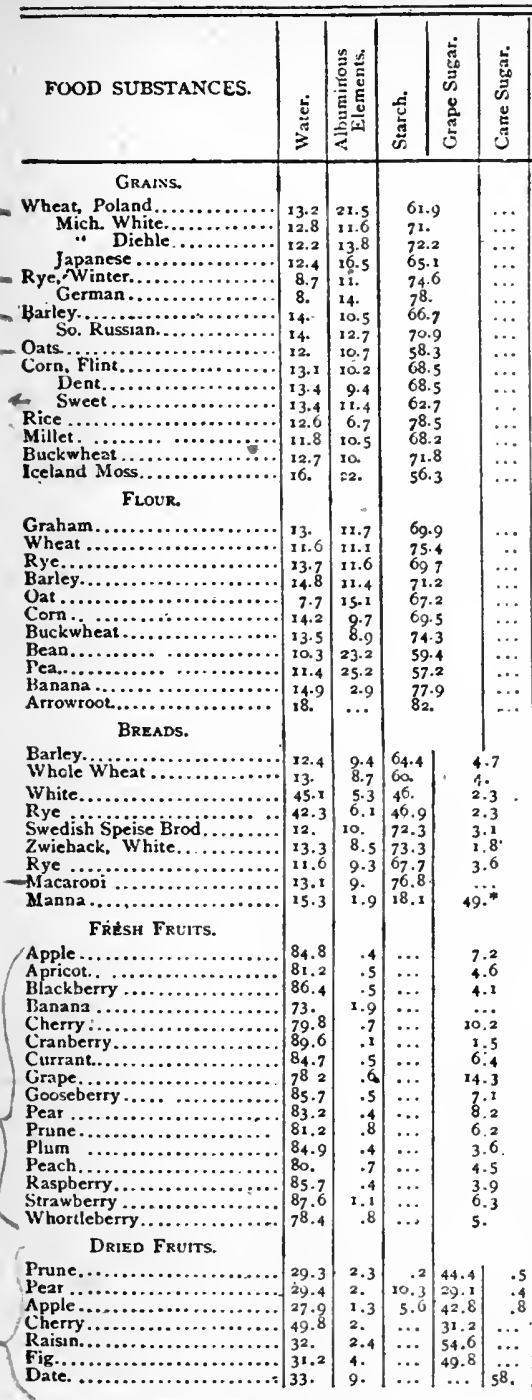

1 Chiefly sugar and starch.

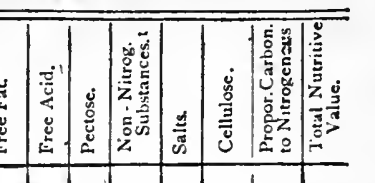




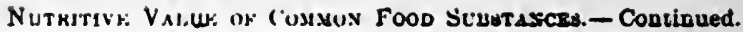

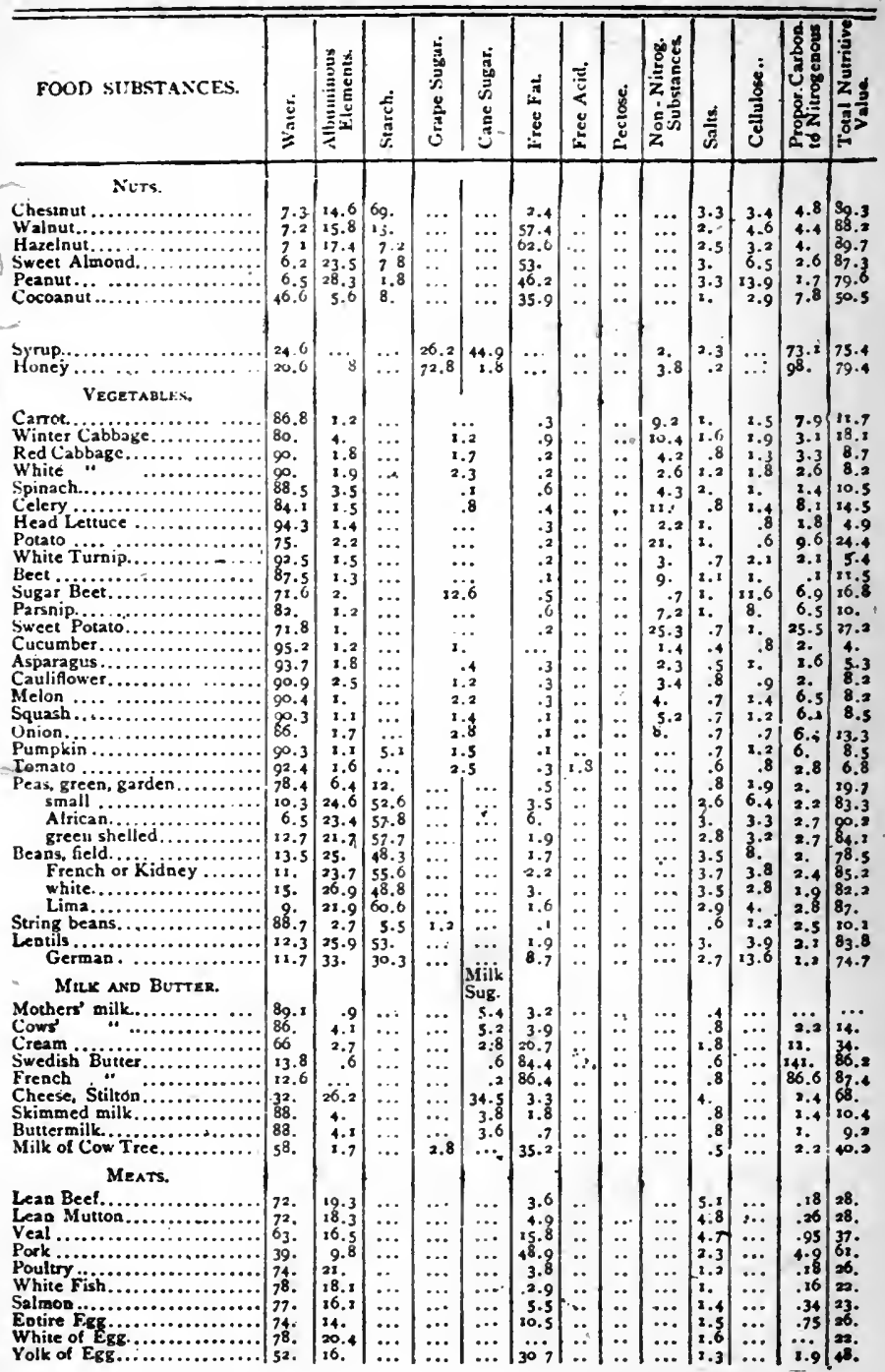




\section{THE DIGESTIVE FLUIDS.}

In studying the organs of digestion, we have learned that there are five digestive fluids; viz., the saliva, the gastric juice, the bile, the pancreatic juice, and the intestinal juice. In the preceding chapter it has been shown that there are five digestible food elements; viz., starch, albumen, ${ }^{1}$ fats, sugars, and salts. Let us now consider the use of each digestive fluid in relation to the various food elements.

Much of the information which has been gained respecting the action of the stomach upon the food is due to the persevering and aceurate observations of Dr. Beaumont, a surgeon in the Amerie:n army, who, in 1822, while stationed in what was then known as Michigan Territory, was called upon to take charge of the case of a young Canadian by the name of Alexis St. Martin, who had been aceidently wounded in the side by the discharge of a musket loaded with shot, at a distance of one yard from his body A portion of flesh as large as a man's hand was torn away from his side, leaving large openings into both the stomach and the chest. The opening communicating with the chest cavity finally closed up entirely, but the opening into the stomach remained. Immediately after his recovery it was about two and one half inches in diameter. Whatever was swallowed passed out through this opening, making it necessary for him to wear a pad to close

1 The word albumen is used to represent all the albuminous elements of the food,-albumen, gluten, casein, etc. 
the opening. In time, nature remedied the difficulty by growing over the opening, upon the inside, a loose fold of membrine which effectually closed it, yet could be easily pushed aside, thus allowing a full view of the interior of the stomach.

Great and important additions to our knowledge of digestion have been made recently by the use of the stomach-tube, a flexible rubber pipe, by means of which digesting food may be removed from the stomach for chemical examination. The facts thus learned gire us more positive and definite information respecting the work of the stomach and the digestive properties of food than has been previously possessed, and throw great light upon the hygiene of digestion.

What the Saliva Digests.-A dry crust of bread, cherred for some minutes, becomes sweet. This is due to the fact that the saliva contains a peculiar principle which, when brought into contact with boiled starch, converts it into malt-sugar, or maltose. In acting upon the starch, the saliva produces first soluble starch, then dextrin, and finally the perfected product, malt-sugar, or maltose. This conversion into sugar constitutes the digestion of starch. It is essential, however, that the starch should be cooked, as the saliva cannot digest raw starch.

What the Gastric Juice Digests.-Pepsin, one of the active principles of the gastric juice, acts upon the albuminons elements of the food, such as eggalbumen, the fibrin of meat, gluten of grains, casein of milk, etc. By its action all of these various substances are converted into one simple substance, known as peptone, which is readily absorbed into the blood, 
while undigested albumen cannot be absorbed to any great extent, and, if absorbed, would be of no use in the system. The gastric juice prepares the food for further digestion by dissolving the substances by which the various elements of the food are held together.

The rennet ferment, or rennin, of the gastric juice coagulates the casein of milk, forming curds, which are afterward dissolved in the gastric juice. The curds formed of cow's milk are much larger and tougher than those formed of mother's milk; hence the difficulty experienced by many persons in eating cow's milk, especially in cases in which the stomach is dilated, and consequently weakened to such an extent that it is not able to break up the hard curds thus formed. When milk is mixed with oatmeal or barley gruel, or other farinaceous substances, the curds formed are more brittle in character ; hence the advantage of adding oatmeal or barley water as a diminuent for milk in many cases. Lime-water has a similar effect, but its long-continued use is objectionable.

Carbolic acid, common salt, and numerous other substances are called antiseptics, because they prevent fermentation or decay. The gastric juice and the bile also possess this remarkable property. A dog was fed. with putrid meat. On being killed, an hour after, the meat, which had been extremely offensive, was apparently perfectly fresh. This property of the gastric juice is exceedingly important, as it prevents decay or fermentation in the stomach before digestion can take place.

What the Bile Digests. - Like the saliva and the gastric juice, the bile digests but a single one of 
the digestible food elements. Its action is wholly upon the fatty portions of the food. If oil and water are shaken together in a bottle, they quickly separate when the shaking ceases. Gum-water and oil, when shaken together, form a milky mixture in which the oil and the water do not separate, and which may be diluted with water the same as milk. The bile acts upon fats in the same manner. Such a mixture is called an emulsion. Under a microscope, the oil of an emulsion is seen to be divided up into very fine drops, or globules.

What the Pancreatic Juice Digests.-The pancreatic juice digests each of the three principal food elements, - starch, albumen, and fats. Amylopsin converts starch into sugar, trypsin changes albumen into peptone, and steapsin makes an emulsion of the fats. The pancreatic juice thus does the work of all three of the preceding digestive fluids, - the saliva, the gastric juice, and the bile.

What the Intestinal Juice Digests.-This fluid possesses but one characteristic digestive property, cane-sugar being ordinarily digested only in the small intestine, and by the action of the intestinal juice. The intestinal juice also digests starch, albumen, and fats, and, together with each of the other digestive fluids, acts upon the salts of the food.

In herbivorous animals, and to a small extent in man, cellulose is digested in the large intestine. 


\section{GENERAL VIEW OF THE DIGESTIVE PROCESS.}

THE digestive process begins the moment a morsel of food enters the mouth, and continues throughout the entire length of the alimentary eanal, or until the digestible portions of the food have been completely digested.

Mastication.- The first act in the digestive process is mastication, or chewing, the purpose of which is to crush the food and divide it into small particles, so that the various digestive fluids may easily and promptly come into contact with every part of it.

Salivary Digestion.- The saliva softens the food, and thus prepares it for the action of the other digestive fluids. It also acts upon the starch, converting a portion of it into sugar.

Deglutition, or Swallowing. - In the act of swallowing, the food does not drop down through an open tube, but is seized by the muscles at the back of the month, and is carried down into the stomach by the action of the esophagus muscles.

Stomach Digestion.- Upon receiving the food, the stomach very soon begins to pour out the gastric juice, which first makes its appearance in little drops, like beads of sweat npon the face when the perspiration starts. As the quantity increases, the drops run together, trickle down the sides of the stomach, and mingle with the food. The muscular walls of the 
stomach contract upon the food, moving it about with a sort of churning action, thoroughly mixing the gastric juice with the food. During this process both the openings of the stomach are tightly closed. The gastric juice softens the food, digests albumen, and coagalates milk. The saliva continues its action upon starch for half an hour after the food reaches the stomach. This action of the saliva is highly important to the process of digestion. It depends, of course, upon the thoroughness with which the food is masticated. If the food is swallowed hastily, it will contain but little saliva, and consequently very little starch will be conrerted into dextrin and maltose, or sugar.; and the person will suffer from starch indigestion.

Action of the Pylorus.-After the food has remained in the stomach from one to three hours, or eren longer, if the digestion is slow or indigestible foods have been eaten, the contractions of the stomach become so vigorous that the more fluid portions of the food are squeezed out through the pylorus, thus escaping into the intestine. The pylorus does not exercise a species of intelligence in the selection of the food, as was once supposed. The increasing acidity of the contents of the stomach causes its muscular walls to contract with increasing vigor, until finally those portions of the food which may be less perfectly broken up, and which the stomach has been unable to digest, are forced through the pylorus.

Intestinal Digestion. - As it leaves the stomach, the mass of partially digested food is intensely acid, from the large quantity of gastric juice which it contains. Intestinal digestion cannot begin until the 
mass becomes alkaline. The alkaline bile neutralizes the gastric juice, and renders the digesting mass slightly alkaline. The bile also acts upon the fats of the food, converting them into an emulsion. The pancreatic juice converts the starch into sugar, digesting both raw - and cooked starch. It also digests fats and albumen. The intestinal juice continues the work begun by the other digestive fluids, and digests cane-sugar.

Other Uses of the Digestive Fluids. - In addition to the -uses which have already been mentioned, several of the digestive fluids possess other interesting properties. The saliva aids the stomach by stimulating its glands to make gastric juice. The gastric juice and the bile are excellent antiseptics, by which the food is preserved from fermentation while undergoing digestion. The bile also stimulates the movements of the intestines, by which the food is moved along, and aids absorption. It is a remarkable and interesting fact that a fluid so useful as the bile should be at the same time largely composed of waste matters which are being removed from the body. This is an illustration of the wonderful economy shown by nature in her operations.

Peristaltic Action.- The food is moved along the alimentary canal, from the stomach downward, by successive eontractions of the muscular walls of the intestines, known as peristaltic movements, which occur with great regularity during digestion.

Absorption.-.. The absorption of the food begins as soon as any portion has been digested. Even in the mouth and the esophagus a small amount is absorbed. The entire mucous membrane lining the digestive canal is furnished with a rich supply of blood-ressels, by 
which the greatcr part of the digested food is absorbed. Absorption is greatly aided by a rhythmical contraction of the villi, which is in effect a sort of pumping action, alternately filling and emptying the lacteal and venous absorbents. The action of the diaphragm in normal breathing also aids absorption by emptying the blood-ressels of the stomach and intestines. During absorption, the digested food is changed into blood.

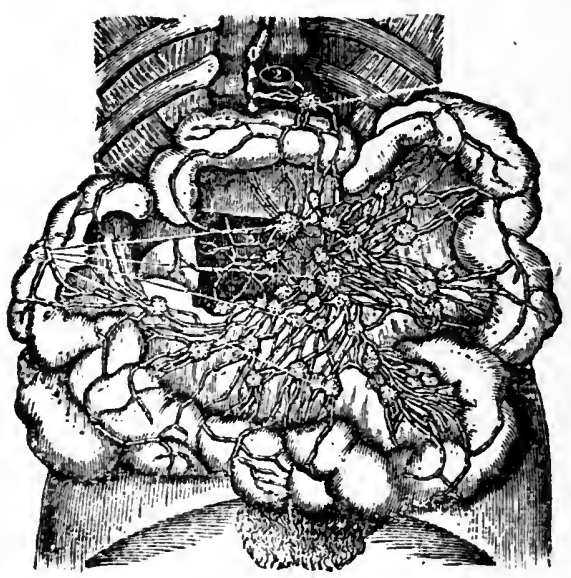

Fig. 10.-The Mesexteric Glaxds axd Lacteals.

The Lacteals. - The walls of the intestines contain eertain small vessels, called lacteals, on account of their white appearance after a meal. This appearance is due to the digested fat which they contain, and which it is their special duty to absorb. The small lacteal vessels unite to form larger ones, all joining at last in one large duct about the size of a crow's quill, called the thoracic duct, which passes upward, and connects with the large vein which returns the bloed from the left arm. (Fig. 10.) 
The Portal Vein.- The veins of the stomach, intestines, pancreas, and spleen all unite to form one large vein, called the portal vein. Instead of emptying, as do the other veins, into the large vein which goes to the heart, the portal vein conveys its blood to the liver, through which it is distributed by a special set of vessels. Afterward it is gathered up by another large vein, and carried to the heart. Thus it appear's that all of the food absorbed by the blood-vessels of the stomach and intestines, constituting the greater part of what is digested, is carried to the liver before entering the general circulation. (Fig. 11.)

\section{Liver Digestion.--} The liver not only secretes a digestive fluid, the bile, but it acts upon the food brought to it by the portal vein, and regulates the

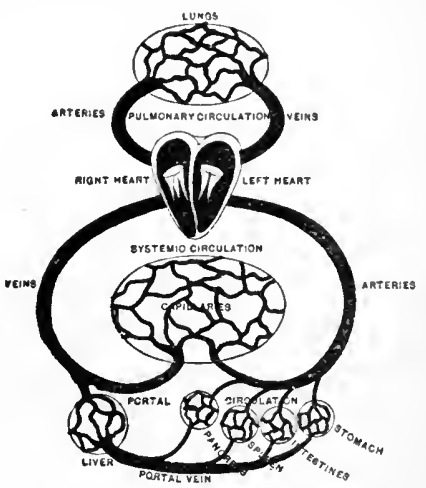

DIAGRAM OF THE CIRCULATORY SYSTEMO.

FIG. 11. supply of digested food to the general system. It converts a large share of the grape-sugar and partially digested starch brought to it into liver-starch, commonly termed glycogen, which it stores up in its tissues. During the interval between the meals, the liver gradually redigests the glycogen, reconverting it into sugar, and thus supplying it to the blood in small quantities, instead of allowing the entire amount formed in digestion to enter the circulation at once. If too large an amount of sugar entered the blood at once, the 
system would be muable to use it all, and would be compelled to get rid of a considerable portion through the kidneys.

The remarkable function by which the liver stores up starch within its tissues is usefully employed as a means of protecting the body from various poisons. When arsenic, mercury, lead, or any other metallic poison is taken into the stomach, any portion absorbed is carried to the liver, which absorbs and retains as much as possible of the poison, and thus protects the rest of the body. The liver treats alcohol and other narcotics in the same manner; and it is doubtless for this reason that the liver suffers so great damage from the use of alcoholic drinks, tobaceo, and other narcotic substances.

\section{The Two Doors - The Liver and the Kid.} neys.-One of the most important functions of the liver is its poison-destroying action. Animal and vegetable poisons, whether originating outside the body or within the stomach and intestines, from the fermentation and decomposition of the food under the action of germs, are in large part destroyed by the liver, or so modified as to greatly diminish their injurious properties. Such portions of these poisons as escape through the liver must be carried out of the body through the kidneys; hence the work of the liver has a very important relation to that of the kidneys.

If the liver fails to do its work properly, the kidneys are overwhelmed with work, and are liable to become disabled. The liver and the kidneys may be aptly compared to two doors. One of the most essential conditions in health, and even in life, is that one of these 
doors should be constantly shut, and the other constantly open. The liver door is, in health, closed against the entrance of poisonous substances, while the kidney door is held wide open, to allow these substances to escape from the body as rapidly as possible. If the liver is overwhelmed with poisonous substances in consequence of errors in diet, it fails to exclude them all, and they enter the circulation, and so crowd upon the kidneys that these organs become obstructed and disabled; and the liver door thus being open, and the kidney door partially closed, poisons rapidly aceumulate within the body, and the most disastrous effects follow. It thus clearly appears why habits of eating have so important a relation to every function of the body.

Breathing and Liver Action.- The circulation of the blood through the liver, and hence all the functions of the liver, are greatly aided by the action of the chest and diaphragm in breathing. When the chest wall is lifted ontward in the act of inspiration, air is not only drawn into the chest, but blood is also drawn toward the heart. Deep breathing is thus a very important means of aiding both the stomach and the liver in their work.

Vagaries of Digestion.- The stomachs of certain persons seem to be possessed of marked peculiarities, as the result of which particular articles of food which agree perfectly well with most people, give rise in them to very unpleasant symptoms, such as oppres. sion or burning, headache, and occasionally slight fever. These symptoms sometimes occur as the result of eating strawberries or honey. They .are, however, much more likely to arise from eating lobsters, oysters, 
and clams. Some people cannot eat buckwheat, and there are cases in which even oatmeal produces a peculiar eruption of the skin. These instances are, however, very rare. The poisonous and sometimes fatal effects resulting from the use of oysters are due to the constant presence in these bivalves of a poison discovered by Brieger - mitylotoxin. 


\section{THE MALADIES OF THE MODERN STOMACH.}

Our forefathers knew comparatively little of the pangs of indigestion, or of the constitutional maladies which grow out of the tissue starvation and systemic poisoning developed from derangement of the digestive functions.

The Causes of Dyspepsia.-An old German proverb reads, "As a man eateth, so is he." This is perhaps a parody upon a more ancient proverb, "As a man thinketh, so is he." Putting both sayings together, we may formulate another equally true, "As a man eateth, so he thinketh." Our bodies are composed of what we eat; nevertheless, the simple eating of good food does not guarantee good tissues, a healthy body, a sound mind, and normal functions. Food must not only be of proper quality, but it must be well digested. Normal digestion is performed unconsciously. An individual who is conscions of the working of his stomach is suffering with a disordered state of that organ. The disorder may be of any degree, from that indicated by a slight feeling of weight or uneasiness occasioned by taking a small excess of food, to the most chronic case of indigestion, exhibiting all the most annoying symptoms of this formidable disease. We say formidable disease, not because it is often a fatal malady, but becanse of its obstinacy, which too often baffles for years the best efforts of those suffer- 
ing from its debilitating effects. In most instances, unfortunately, the efforts of the patient and of his advisers, though earnest and persevering, are unavailing, because not well directed. With this, as with all other diseases, the first step toward recovery lies in the discovery and removal of the causes of the disease. Having considered the process of digestion as it occurs in health, and the conditions required for healthy digestion, we shall now be better able to appreciate the influence which various violations of the laws of health relating to this function may exert upon it.

Healthy digestion depends upon a maintenance of the following conditions in relation to the stomach :-

1. The secretion of gastric juice in proper quantity and of good quality.

2. The prompt absorption of the digested food substances.

3. Normal muscular activity of the stomach, by which the organ is emptied of its contents in due season, or by the end of two or three hours after a meal.

4. The maintenance of an aseptic condition of the stomach; or, in other words, a condition in which the stomach is free from microbes, or germs.

Dyspepsia may result from a departure from the normal standard in relation to one or all of the above conditions. The gastric juice may be either deficient or too abundant. Absorption is never too rapid, but it may be greatly diminished, as in gastric catarrh.

Muscular activity may be either deficient or excessive. If deficient, the food is retained for too long a time in the stomach, resulting in irritation of the mucous membrane from too prolonged contact with the 
acid gastric juice, and in some cases even giving rise to ulceration. This delay in the digestive processes also allows opportunity for the action of germs upon the food, thus causing fermentation and putrefaction. Professor Bouchard, of Paris, has shown that if food is retained in the stomach for more than five hours, putrefactive changes take place. Fermentation may begin much sooner than this. Muscular action may be excessive, in which case the food will be hurried out of the stomach into the intestines before the process of gastric digestion is completed, thus giving rise to irritation in the intestinal canal from contact with food which has not been properly prepared for the processes which occur in this part of the digestive apparatus.

Healthy gastric juice is capable of keeping the stomach in a perfectly aseptic condition; that is, destroying all classes of germs which are likely to find entrance to the stomach. If the gastric juice is deficient in quantity or quality, it may fail to do its work as a disinfecting agent, so that germs may develop in the stomach, and becoming embedded in the mucus which covers its lining, permanently establish themselves there. Different classes of germs may thus become active in the stomach, either singly or in combination. Such symptoms as acidity, or sour stomach, heartburn, coated tongue, a bad taste in the mouth, distention of the stomach, eructations of gas, etc., are due to the presence of germs in the stomach, and their action upon the food elements. Germs do not find lodgment in the stomach when the food is digested thoroughly, and promptly absorbed or passed 
onward into the intestine. The special causes now to be mentioned are more or less active as agents prodnctive of dyspepsia, in proportion as they disturb the essential conditions of good digestion above enumerated.

Errors in Diet. - There is no room to doubt that errors in diet, in the manner of eating, or in quantity and quality of food, are by far the most active causes of indigestion. By asking a dyspeptic how ho eats, what he eats, and when he eats, it is usually easy to discover the cause of his suffering; and by inducing him to form correct habits in these three particulars, a cure will be effected in nine cases out of ten. In many and perhalps most cases, however, other adverse influences of various sorts serve to some extent to complicate the digestive disorder, and to intensify the effects arising directly from the causes named; hence we shall not confine our investigation of the causes of indigestion to the class of causes mentioned, exclusively, but shall also notice those which more remotely operate in this direction, first, however, calling attention to dietetic errors as the most common and most powerful causes of the disease under consideration.

Hasty Eating.-- The evils resulting from hasty eating may be enumerated as follows :-

1. From deficient mastication, the food is not properly divided, so that the digestive juices cannot gain access to its various elements.

2. By being retained in the mouth too short a time, an insufficient amount of saliva is mingled with it, so that salivary digestion cannot be properly performed. As the saliva is also a stimulus to the secretion of 
gastric juice, stomach digestion must necessarily be imperfect.

3. Again, if the food enters the stomach in a coarse, unmasticated state, it may act as a mechanical irritant to the delicate lining of the stomach, and thus occasion congestion and gastric catarrh, one of the most common disorders of the stomach, and one which is often very obstinate in its nature.

The best remedy for the habit of hasty mastication is the use of dry food. The importance of this is well shown by a series of experiments conducted by the author for the purpose of determining the amount of saliva produced in masticating dry food as compared with moist and liquid foods. The results were as follows :-

A piece of paraffin chewed for five minutes produced two thirds of an ounce of saliva.

One ounce of granose, a dry food prepared from wheat, increased in weight to two ounces. The addition of pepper and salt to the granose slightly decreased the amount of saliva produced. The addition of vinegar still further diminished the secretion.

One ounce of moist bread chewed for five minutes caused the production of one ounce of saliva.

One ounce of raw apple produced one and onefourth ounces.

An ounce of water produced but one tenth of an ounce of saliva, or about one sixth as much as a piece of paraffin, and one thirtieth as much as an ounce of granose.

One ounce of milk was slightly more active in producing saliva than the same amount of water. 
An ounce of pea soup chewed for five minutes produced twice as much saliva as did water, but only one third as much as paraffin, and one tenth as much as granose.

The use of "slops," so common in the United States, and to a great extent also in other countries, is one of the most serious of dietetic errors. The American eats in a hurry, rinses down his food with copious draughts of tea, coffec, iced water, iced milk, or iced tea; and in consequence the salivary glands are not stimulated to proper activity, so that the amount of saliva produced is altogether inadequate to digest the starchy elements of food in the acid medium of the stomach contents, and the small amount produced is so diluted that its efficiency is greatly impaired. What wonder that starch indigestion is coming to be an almost universal complaint, as shown by acidity, eructations of gas, flatulence, and a great variety of stomach disturbances, to escape from which multitudes are continually swallowing quantities of magnesia, soda, neutralizing cordials, and alkaline mineral waters of various sorts, together with malt extracts and other digestants!

The inability to digest starch is doubtless one of the great causes of the inordinate consumption of beef and other animal products to which the English-speaking race has come to be addicted, as a method of escaping the pangs of starch indigestion.

The abundant provision made in the human body for the digestion of starch, - first, the saliva ; second, the bile and pancreatic juice; third, the intestinal juce ; and finally, the liver, - is evidence that nature 
intended man to subsist largely upon farinaceous foods. The arguments of the "natural food" advocates, who insist that man should live upon fruits and nuts, are based, not upon physiological facts, but upon the morbid experiences of the disciples of this doctrine. The writer had an opportunity, a year or two ago, to examine the stomach fluid of one of the most earnest and stalwart advocates of the fruit-and-nut diet, and the stomach was found greatly dilated, and almost completely inert.

I have cured many scores of chronic and very obstinate cases of dyspepsia by simply requiring the patient to subsist upon a dry diet, whereby he was compelled to thoroughly masticate his food. A favorite prescription with the writer, which is applicable in most eases of indigestion, is one or two ounces of dry granose eaten at the beginning of each meal. This introduces into the stomach an abundant quantity of saliva, - probably from four to six ounces in most cases,- and insures efficient starch digestion.

The dextrin and maltose produced by the action of the saliva upon the starch are exactly what the stomach needs to stimulate it to healthful activity, whereby a proper quantity and quality of gastric juice will be produced.

Diseased Teeth.-Ulceration of the teeth is a very common ailment. Decay of a tooth is exactly analogous to ulceration in other parts of the body. This destructive action upon the teeth is due to the presence of germs in the mouth, which produce chemical substances of such a nature as to dissolve the 
enamel, and thus expose the inner portion of the teeth to the action of destructive microbes.

Defective teeth, by interfering with the complete and thorough mastication of food, seriously impair the digestion. On the other hand, impairment of digestion, with its consequent perversion of the secretions, is a very common cause of decay in the teeth. Many persons suffering from disorders of digestion cannot hope to recover without giving attention to the teeth, that being necessary as the first step toward reforming the condition of the stomach. If possible, the natural teeth should be preserved by filling when decayed, and by such other measures as any good dentist will recommend.

The microbes harbored by decaying teeth are swept into the stomach in drinking or eating, and there set up fermentative and putrefactive processes, whereby the food elements are converted into poisonous substances. Some of these poisons are possessed of such strong odors as to taint the breath. Decaying teeth may also be the means of introdueing into the body such destructive microbes as the germs of consumption, and others giving rise to serious and even fatal disease.

Decay of the teeth is generally the result of lack of use and of cleanliness. By the use of dry food as previously stated, the tecth are polished and kept free from the colonies of germs which settle upon them when they are not scoured by attrition of hard substances. Meat eating is especially favorable to the destruction of the teeth. The small particles of meat 
which collect between the teeth encourage the development of germs of the most destructive character. Preservation of the teeth requires habitual and scrupulous cleanliness. They should be thoroughly cleansed before and after each meal,_- before the meal for the purpose of protecting the stomach from the germs which accumulate in the mouth from the air, and lodge about the teeth ; and after the meal for the purpose of removing every particle of food, so that the growth and development of germs which subsist upon remnants of food left behind in the mouth, shall not be encouraged.

It is well to employ some suitable antiseptic as well as a cleansing agent for the teeth, but many harmful substances are recommended for this use. The writer has had an antiseptic dentifrice prepared, the basis of which is an extract of the famous soap-tree of South America, and cinnamon oil. The first of these ingredients is as cleansing as soap, and avoids the impurities and chemical substances found in it. Oil of cinnamon is one of the best antiseptics, preventing the growth of germs even when not used in sufficient quantity actually to destroy them.

The rapid decay of the teeth in civilized countries, which is principally due to the excessive use of meats and of soft foods, as mushes, soups, bonillons, etc., has in our time led to a full realization of the dream of Giles Corey, who was put to death for witchcraft at Salem nearly two hundred years ago, and who wrote :-

"I saw a man pull all his teeth,

It took him but a minute;

He op'd his mouth and put them back;

I thought 'e deuce was in it.:" 
The tartar which sometimes accumulates upon the teeth is due to the action of germs. Tartar should never be allowed to remain, - indeed, the teeth should be so often and so carefully cleansed as to prevent the slightest accumulation of tartar. When allowed to grow and remain, tartar separates the gum from the teeth, and produces an unhealthy state which often causes the loss of the teeth. Fig. 12 shows human teeth on which are formations of tartar. In Fig. 13 are shown the same teeth with the tartar removed. The teeth of Orientals, negroes, and in fact of nearly all primitive people, are

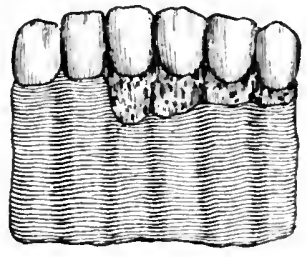

Fig. 12. HUMA: TeEtil CovereD WITH TARTAR.

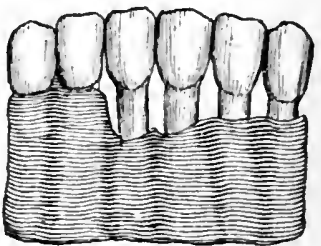

Fig. 13.-HuMan TeEth INJURED BY TARTAR.

generally remarkably sound, because of their simpler diet and small use of meat.

If the natural teeth cannot be saved and made serviceable, they should be replaced by artificial ones. No one can hope to preserve good digestion while munching food with toothless gums, or subsisting on a dietary that does not require the use of teeth.

Drinking at Meals. - In addition to the evils which it occasions directly, hasty eating induces the drinking of large quantities of hot or cold liquids to wash the food into the stomach. Thus two evils are associated. Too much liquid of any kind is prejudicial to digestion, because it delays the action of the gastric 
juice, weakens its digestive qualities, and overtasks the absorbents. In case the fluid is hot, it relaxes and weakens the stomach. If it is cold, it checks digestion by cooling the contents of the stomach to a degree at which digestion cannot proceed. Few people are aware how serious a disturbance even a small quantity of cold water, iced cream, or other cold substance, will create when taken into a stomach where food is undergoing digestion. This process cannot be carried on at a temperature less than that of the body, or about $100^{\circ} \mathrm{F}$. Dr. Beaumont observed that when Alexis St. Martin drank a glassful of water at the usual temperature of freshly drawn well-water, the temperature of the food undergoing digestion fell immediately to $70^{\circ}$, and did not regain the proper temperature until after the lapse of more than half an hour.

Of course the eating of very cold food must have a similar effect, making digestion very tardy and sTow. If any drink at all is taken, it should be a few minutes before eating, so as to allow time for absorption before digestion begins. If the meal is mostly composed of dry foods, a few sips of warm or moderately hot water will be beneficial rather than otherwise, taken either at the beginning of the meal or at its close. The habit of drinking during the meal should be discontinued wholly, and especially by those whose digestive powers are weak. If the diet is of proper quality, and the food is well masticated, there will be little inclination to eat too much. When the food is rendered fiery with spices and stimulating condiments, it is no wonder that there is an imperious demand for water or liquid of some kind to allay the irritation. 
Eating too Frequently. - One of the most pernicious customs of modern life is that of frequent meals. This habit is seen in its extreme development in England, five meals a day, including lunches, being there considered none too many. The idea seems to prevail that the stomach must never be allowed to become empty under any circumstances. In this country, three meals a day is the general custom, though more are sometimes taken.

Healthy digestion requires at least five or six hours, and one or two hours for rest before another meal is taken. This makes six hours necessary for the disposal of each meal. If ordinary food is taken at shorter intervals than this, the stomach must suffer disturbance sooner or later, since it will be allowed no time for rest. Again, if a meal is taken before the preceding meal has been digested and passed from the stomach, the portion remaining, from its long exposure to the influence of warmth and moisture, is likely to undergo fermentation, in spite of the preserving influence of the gastric juice. Thus the whole mass of food is rendered less fit for the nutrition of the body, and what is still more serious, the stomach is liable to suffer permanent injury from the acids developed.

Too frequent eating occasions too long contact of the acid contents of the stomach with the gastric juice, which produces catarrh and ultimately ulceration of that organ. Frequent eating is often resorted to as a relief from what is commonly termed faintness, or an "all gone" feeling in the stomach. The fact that eating gives a respite from this sensation is taken as good evidence that it is the proper remedy ; but, at the best, 
only temporary relief is obtained, and that at the expense of aggravating the difficulty. Food affords relief simply by separating the irritated mucous surfaces of the stomach, and diluting its acid fluid. The proper remedies for these unpleasant symptoms are given elsewhere in this work.

Eating between Meals. - This is a gross breach of the requirements of good digestion. The habit many have of eating fruit, confectionery, nuts, sweetmeats, etc., between meals, is a certain cause of dyspepsia. No stomach can long endure such usage. Those who. indulge in this manner usually complain of a poor appetite, and wonder why they have no. relish for their food, strangely overlooking the real cause, and utterly disregarding one of the plainest laws of nature.

This harmful practice is often begun in early childhood. Indeed, it is too often cultivated by mothers and the would-be friends of little ones, who seek to please and gratify them by presents of confectionery and other tidbits of various sorts. Under such indulgence, it is not singular that so many thousands of children anmually fall victims to stomach and intestinal diseases of various forms. In great numbers of cases, early indiscretions of this kind are the real canse of fully developed dyspepsia in later years. What a sad thought that the lives of such persons have been modified in their usefulness, and their whole characters more or less depraved, by the morbid influence of disordered digestion.

Irregularity of Meals. - Another cause of dyspepsia, which is closely related to the ones just mentioned, is irregularity respecting the time of meals. 
The linman system seems to form habits, and to be in a great degree dependent upon the performance of its functions in accordance with the habits formed. In respect to digestion this is especially observable. . If a meal is taken at a regular hour, the stomach becomes aceustomed to receiving food at that hour, and is prepared for it. If meals are eaten irregularly, the stomach is taken by surprise, so to speak, and is never in a proper state of readiness for the prompt and perfect performance of its work. The habit which many professional and business men have of allowing their business to intrude upon their meal hours, frequently either wholly depriving them of a meal or obliging them to take it an hour or two later than the usual time, ultinately undermines the best digestion. The hour for meals should be considered a sacred one, not to be intruded upon exeept under some unusual cireumstance. Eating is a matter of too momentons importance to be interrupted or delayed by matters of ordinary business or convenience.

The habit of regularity in eating should be cultivated early in life. Children should be taught to be regular at their meals, and to take nothing between meals. This rule applies to infants as well as to older clildren. The practice of feeding the little one every time it cries, results in most serious injury to its weak digestive organs. An infant's stomach, though it needs food at more frequent intervals than an adult's, - every two to four hours, according to its age, - requires the same regularity which is essential to the maintenance of healthy digestion in older persons. The irregularity usually practiced is undoubtedly one of the greatest 
causes of the large number of deaths among infants from disorders of the digestive organs shown by our mortuary records.

The action of the digestive organs, like that of all other organs of the body, is rbythmical. The discharge of the alimentary residue, which constitutes the fecal matters, noirmally occurs after the first meal of the day. It is the result of the peristaltic movements set up by the introduction of food into the stomach. By this increased activity of the alimentary canal the fecal matters resting in the upper portion of the colon are moved downward into the rectum, thereby provoking a desire for evacuation of the bowels. By this means, the activities set up by each meal move the contents of the intestine to their appropriate station, resulting, in healthy persons, in the discharge of the alimentary residue from the body at a stated hour each day. If a meal is omitted, or if meals are taken at irregular hours, this rhythmical action is broken up, and constipation is the natural result.

It thus appear's that eating at too frequent intervals is not the only evil in the way of irregularity in eating. It is far better, however, to omit a meal than to introdnce into the stomach a new supply of food before that already contained in it has been properly digested, and the organ given an opportunity for rest.

The Proper Number of Meals. - The number of meals which should be taken by a person in health depends somewhat upon the habits of the individual, his occupation, the number of hours he labors, etc. There is good reason to believe that for a large proportion of those who now take three to fire meals a 
day, two would be much better. According to Hippocrates, the ancient Greeks ate but two meals a day. The same was true of the ancient Hebrews and Persians. This is also the custom of the natives of India, of South America, and of many semi-civilized nations. Among savage tribes, one meal a day is the prevailing custom. The Eskimo walrus hunter sets out in his kajak: on a day's hunt at the break of day, but eats nothing until after he returns with his prey, just before sunset.

The modern frequency of meals is the outgrowth of a gradual losing sight of the true purpose of the eating of food, and of regarding the gratification of the palate, instead of the nourishment of the body, the chief object to be attained. That the system can be well nourished upon two meals a day is beyond controversy, seeing that not only did our vigorous forefather's require no more, but that hundreds of persons in modern times have adopted the same custom without injury, and with most deeided benefit to themselves. Students, teachers, elergymen, lawyers, and other literary and professional men are especially benefited by this plan. The author has followed the plan for more than thirty years, and with great satisfaction The special advantages gained by it are: 1 . The stomach is allowed a proper interval for rest. 2. Sleep is much more recuperative when the stomach is allowed to rest with the balance of the body. 3. Digestion cannot be well performed during sleep.

Dujardin-Beaumetz, an eminent French physician, Bouchard, and other well-known European authorities, insist that seven hours is the proper length of time to be allowed for the digestion of each meal. If this 
plan is followed, seven hours being. allotted to the digestion of each meal, and the proper length of time allowed to elapse before going to sleep after the last meal, it will be found impossible to make any arrangement by which opportunity can be secured for the necessary eight hours' sleep at night. Not more than two meals can be taken when a person complies with all the laws of health.

If more than two meals are suited to any class, it is those who are engaged for twelve or more hours per day in severe mental and physical labor. Such per'sons are better prepared to digest a third meal than those whose occupation is mental or sedentary, and they may at least take it with less detriment, though a third meal is not needed, even for such, provided the two meals are taken at suitable hours. For many years, the practice at the Battle Creek Sanitarinm has been to furnish its guests with two regular meals daily, the first at 8 A. м., the second at 3 р. м. The employees, numbering at the present writing, something more than six hundred, are also furnished with but two meals, at 6 A. м. and 1:30 P. M. The universal testimony of all who have become accustomed to these hours for eating is that more work and better work can be accomplished than when three meals are taken. In cases requiring more than two meals, as when liquid food or only small quantities of food, can be taken at a time, we find it wise to supply two minor meals; at $12 \mathrm{~m}$. and 7 P. M.

\section{Late Suppers and Six-o'clock Dinners.-} Eating late at night, when the muscular and nervous systems are exhausted by the labor of the day, and 
retiring to rest soon after, is one of the most positive dyspepsia-producing habits of modern times. A sleeping stomach is a slow one. Sccretion must of necessity be defieient in both quantity and quality, owing to the exhausted condition of the system; and with the further obstacle afforded to prompt digestion by the slowing of the vital operations during sleep, it is almost impossible that there should be other than disturbed digestion, and restless slecp in consequence. It is under these cireumstances that people often suffer with obstinate insomnia, bad dreams, nightmare, and similar troubles, from which they arise in the morning unrefreshed and unrecuperated by nature's sweet restorer, the work of assimilation, by which repair takes place, having been prevented by the disturbed condition of the nerves.

No food onglit to be taken within four hours of retiring. This will allow the stomach time to get the work of digestion forwarded sufficiently to enable it to be carried on to completion without disturbance of the rest of the economy. If a third neal is taken, it should be very light, preferably consisting of ripe fruit and simple preparations of grains. The custom which prevails in many of the larger cities, of making dinner the last meal of the day, eating of foods the most hearty and difficult of digestion as late as six or even eight o clock, is one that ought to be discountenanced by physicians. It should be tolerated only by those who convert night into day by late hours of work or recreation, not retiring until near midnight. But in such cases a double reform is needed, and so there can be no apology offered for this reprehensible practice, on any physiological grounds. 
It should be remembered that the process of digestion is not complete when the food leaves the stomach. Recent observations made upon a man having a fistulous-opening at the junction of the small intestine with the colon, have shown that fourteen hours elapse from the time food is eaten until it enters the colon, when the process of digestion is completed, the colon being chiefly a reservoir where the digested food is retained for absorption.

Eating when Exhausted.-This is a most certain cause of derangement of digestion, and one to which a very large number of cases of dyspepsia may be traced. The third meal of the day is almost always taken when the system is exhausted with the day's labor. The whole body is tired, the stomach as well as other parts of the organism. The idea that by the taking of food the stomach or any other part of the system will be strengthened, is a mistake. An eminent writer on indigestion says very truthfully, "A tired stomach is a weak stomach." When the stomach feels "weak and faint," rest is what is demanded, and is the only thing that will do it good; yet many people insist on putting more food into it, thus compelling it to work . when it ought to be allowed to remain inactive until rested. The arm wearies by constant exercise, and so does the stomach, which, like the arm, is active through its muscular structure. Both secretion and muscular activity are greatly lessened in a tired stomach, and the habitual disregard of this fact cannot but be disastrous to the best digestion.

Violent exercise either just before or just after eating is a hindrance to good digestion. When the exer- 
cise is taken just before the meal, the stomach is left too tired to do its work properly; and when taken immediately after eating, the vital energies are diverted to other parts, and the stomach is thus robbed of its necessary share. An English physiologist performed an experiment which well illustrates the truth of this position. Having fed a dog his usual allowance of meat one morning, he took him out upon a fox hunt, and kept him racing over the country until night, when, having killed the animal, he examined his stomach at once, and found the meat in the same condition in which it had entered the stomach, no digestion having taken place. In another dog, fed with the same kind of food, but left quiet at home, digestion was found to be complete.

The hurry and press of business among Americans - is allowed to override every consideration of health. It seems never to enter the mind of the average business man that any time is required for digestion. Rushing to his dinner from the plow, the workshop, or the counting-room, he swallows his food with all possible dispatch, and hurries back to his work again, begrudging every moment spent in meeting the requirements of nature. Many years agc it was customary in Edinburgh to suspend all business for two hours in the middle of the day, so as to allow ample time for meals. A similar custom once prevailed in Switzerland; but probably such a sensible custom is now considered too old-fashioned to be tolerated.

It should be remarked that severe mental labor immediately before or after and especially during meals, is eren more injurious than physical employment. The 
habit many business men have of anxiously scanning the newspapers during their meals, and while going to and from their places of business, is a bad one. At least a full hour should be taken for the midday meal ; and if an hour's rest can be secured before eating, improved digestion will well repay the time spent in reinforcing the vital energies. For persons of weak digestion, the rest before eating is almost indispensable.

Recent experiments have shown that the muscular strength is increased very soon after eating. This must be due to the digestion and absorption of the starch, whereby the muscles are furnished a fresh supply of glycogen, which is their source of energy. It is, therefore, admissable that a very small amount of food be taken by an exhausted person, but it should be of a kind which is easily digested, and the quantity should be very small. Nitrogenous food, such as meat and eggs, is especially detrimental to a person in this condition. Thin, well-boiled gruel or rice water, a little rice, a crust of bread well chewed, a cup of vegetable broth, a bunch of grapes, an orange, or a bit of ripe fruit of some other kind, are most suitable for this purpose.

Sleeping after Meals. - While rest from accustomed exercise after eating is important, it should be noted that sleep at this time is equally as bad as vigorous exercise of either mind or body. Good digestion cannot take place during sleep. While it is true that digestion is an involuntary act, it shonld be recollected that it is dependent upon the activity of the nervous system for its proper performance. The same nerve 
which secures activity of the respiratory organs, - the pneumogastric, - controls the muscular activity of the stomach and the intestines. During sleep, from the lessening of nerrous activity, both the respiration and the circulation are greatly lessened in vigor. It is but reasonable to suppose that the activity of the digestive organs is decreased at the same time, being controlled by the same nerves. Actual experiment shows this to be true. Most people who lie down and sleep an hour or two soon after taking food, awake feeling anything but refreshed. The suspension of the process to a considerable degree during sleep causes imperfect digestion, with its numerous unpleasant symptoms. In the case of old people, it may sometimes be beneficial, or at least not harmiful, to secure a few minutes' sleep after eating, before digestion is well begun; but it must not be long continned.

In order to secure the best conditions for digestion after eating, an individual should take gentle exercise of some kind, as walking, or carriage or horseback riding. While violent exertion seriously interrupts the digestive proeess, a moderate degree of physical exercise facilitates it by increasing the activity of the glands by which the gastric juice is formed. It is probable that gentle exercise also encourages digestion by stimulating the movements of the stomach.

Too Many Varieties of Food.-Many dyspepsias arise from the eating of too many kinds of food at the same meal, another growing custom of modern times which deserves to be severely condemned. At great dinners in honor of distinguished personages, when friends are to be entertained, and in the majority 
of well-to-do families as a general custom, the eaters are tempted to gluttony by having presented to their palates a great variety of complicated dishes. On the occasion of the giving of a great dinner, more than a hundred dishes are sometimes served in successive courses. Such gormandizing soon breaks down the most vigorous digestive organs, since it adds to the labor of digesting food which is improperly cooked, a larger variety than the digestive juices are capable of bringing into a fit state for absorption.

Food Combinations. - Careful experiments have shown very clearly that different classes of food require a particular quality of digestive juices for their digestion. For instance, a gastric juice that will digest animal food the best, is inferior for the digestion of vegetable food, and vice versa. The obvious conclusion to be drawn from this fact is that the simpler the dietary, the more perfectly will the digestive process be performed. For persons whose digestion is naturally weak this is a matter of especial importance. The following table represents the best and worst food combinations :-

\section{GOOD COMBIXATIONS.}

Grains and Fruits.

Grains and Neat, or Eggs.

Grains and Milk.

Grains and Tegetables.

FAIR COMBINATIONS.

Grains, Sweet Fruits, and Milk. Meat and Vegetables.

BAD COMBIFATIONS.

Fruits and Vegetables.

Milk and Meat.

Milk and Vegetables.

Those foods agree best whose chief constituent elements are digested by the same fluid, in the same part 
of the alimentary canal, and in about the same length of time. Vegetables contain a great amount of coarse, woody structures, which are retained in the stomach a long time before they are sufficiently broken up to be easily digested in the intestines. Fruits, on the other hand, remain but a short time in the stomach. The large amount of saccharine matter which fruits contain, makes them likely to set up fermentation in the stomach, if retained too long. Acid fruits are also likely to delay starch digestion. This is another reason for their interference with vegetables, the starch of which is rather more difficult of digestion than that of grains.

Milk and regetables are likely to disagree, for the reason that milk, when taken by itself, is retained in the stomach but a short time, its digestion being carried on chiefly in the small intestine. Milk and meat are a bad combination for the same reason. Meat requires long digestion in the stomach, whereas milk, when taken by itself, is quickly passed on, to be digested by the pancreatic juice. When taken with meat or vegetables, milk, being long retained in the stomach, undergoes fermentation, resulting in sour stomach, biliousness, and various other unpleasant symptoms.

If the bill of fare taken at a single meal were confined to three or four articles of food, there would be fewer dyspeptics scanning the newspapers for some patent nostrum to "aid digestion."

Hot or Cold Bathing after Meals.-Especial mention should be made of the injury to the digestive organs quite certain to result from taking either a hot or a cold bath soon after eating. Few people are aware of the danger of laying the foundation for years of dis- 
comfort in this way. If the bath be a hot one, the stomach will be deprived of the blood necessary to support the rapid secretion of gastric juice for the digestion of the food, by its being drawn to the surface of the body by the sudden relaxation of the capillaries and small vessels of the skin. A cold bath, on the other hand, or any sudden exposure to cold, may, by causing contraction of the blood-vessels of the surface of the body, cause sudden congestion of the stomach, which is equally fatal to good digestion. Very nearly the same danger exists from bathing just before a meal.

The practice very common among boys and young men, of going into the water in the summer-time, regardless of the state of the digestion or other conditions of the body, is a bad one. With many it is a very nsual practice two or three times a week, if not oftener, to go into the water immediately after the evening meal, not even allowing time for the work of digestion to become established. No bath involving any considerable portion of the body should be taken within two hours after a meal, except by the advice of a physician.

Errors in Quantity of Food.--If errors in the manner of taking food are active causes of indigestion, mistakes in quantity are still more potent in this direction. It should be noted, however, that error's of this class are very closely connected with others in the manner of eating, and in the quality of food taken. It is generally true with physical as well as moral transgression, that one bad habit invites another ; and especially is this the case in reference to dietetic errors. A person who eats too fast is likely to eat more than is nec- 
essary; and the same is true if too large a variety of food is partaken of, or food rendered exciting and stimulating by seasoning with irritating condiments. Overeating. - Intemperance in eating is, in the opinion of the writer, responsible for a greater amount of evil in the world than is intemperance in drink. Indeed, it can be clearly shown that intemperate eating is, in the first place, one of the most potent causes of intemperance in drink, and also that it is one of the obstacles in the way of the reformation of those who have become the victims of alcoholic intemperance.

The extent to which overeating is sometimes carried is almost incredible. Travelers in the Arctic regions report that it is not an uncommon thing for a Laplander or an Eskimo to devour a large portion of a small sheep at a single meal. A German authority reports the case of a hysterical girl eighteen years of age, who ate regularly twelve times a day, consuming from twenty-four to twenty-six pounds of food. In another case a woman who was seized with a peculiar form of morbid appetite, known as bulimia, in forty-five minutes ate twentythree eggs, and drank three pints of milk and two pints of wine.

But if we may beliere the statements of historians, gluttony is by no means a modern vice. Indeed, there is quite good ground for concluding that orereating, while a very general fault, is rarely, if erer, at least among civilized nations, carried to the enormous excess in which some of the luxurious Roman emperors indulged.

The evil consequences of excess in eating are at first simply imperfect digestion, the overtaxed organs being 
unable to accomplish the complete digestion of the alimentary mass. In consequence of the delay which occurs, changes take place by which acids are developed that irritate the mucous membrane, together with gases by which the stomach is distended, and its muscular walls weakened and partially paralyzed. In course of time, inflammation of the gastric membrane is developed, and permanent dilatation of the stomach occurs.

This condition is one which cannot usually be entirely cured, and which gives rise to a great variety of ailments in addition to the discomforts connected directly with the stomach itself. These are more fully pointed out elsewhere. Dilatation of the stomach often originates very early in life. The writer has found the organ dilated to more than its normal size in children scarcely a dozen years of age, and in many cases has been able to trace this condition found in adults back to early childhood.

An individual who overeats will at first be likely to accumulate flesh quite rapidly; but very soon the digestion becomes so much disturbed that no gain takes place, and, indeed, the patient not infrequently becomes considerably emaciated even while daily taking large quantities of food. When the opposite is the case, the blood is filled with crude, imperfectly elaborated material, which, when absorbed, fills the system with poisonous substances. At first the liver is able to exclude these to a considerable extent, but after a time the energy of this patient organ is entirely exhausted, and the whole system suffers in consequence.' Biliousness and the various conditions usually attributed to torpid- 
ity of the liver are generally due to poisoning of the system by toxic substances absorbed from the alimentary canal, which may be either the result of the putrefaction of food in a dilated stomach or colon, or the products of imperfect digestion.

Excessive eating also occasions injury to the health by prodncing an excessive fulness of the blood-vessels, thus incurring the risk of rupture within the brain, and resulting paralysis. Other equally serious mischiefs may arise from the accumulation in the system of a greater quantity of nutritive material than can be utilized, which occasions general clogging and obstruction of all the bodily functions, and imposes an enormous burden upon the kidneys in the elimination of the unusable material.

Luting too Little.-A far less common fault than the one last mentioned, is eating too little. The instances that occur are usually in the cases of those who have attempted to subject themselves to a rigid dietetic regimen for the prevention or cure of disease, and who, from having only a partial view of the subject, entertain extreme notions. By the weakening of the system which necessarily occurs when an insufficient amount of nutriment is received, the stomach also becomes weak and debilitated, its secretions and muscular efforts being greatly impaired in both quantity and quality.

How Much Should a Person Eat? - Thousands of times has the writer been asked this question. The only reply that can be made to this inquiry is, Just so much as the system needs and the digestive organs can digest. In general, an individual may take as much food as he can digest ; but often there are con: 
ditions in which he cannot digest as much as he really needs. For instance, when an individual is called upon to exert all his energies of brain and muscle, to strain every nerve to its utmost, to compass a certain object of great importance, to cope with an emergency, he may be, for the time being, quite unable to digest sufficient food to make good the waste that must necessarily occur. He will lose flesh and strength under such circumstances ; and often a failure of the appetite at such a crisis indicates the inability of the stomach to digest, on account of the deficient secretion of gastric juice. It is in this way that persons who are for a time called upon to make great exertions often break down their digestion. Thinking that they need abundance of nutriment, which is true, they eat as heartily as when required to perform only their ordinary work, not considering their diminished power to digest and appropriate food, and in a short time find their digestive organs unable to digest well even a small amount of food. There is little doubt that this is what causes many lawyers, physicians, and other professional men to break down. If, when called upon to do a large amount of extra work, the person would lessen the quantity of food eaten, instead of increasing it, he would conserve his vital forces much more than by pursuing the opposite course. When required by a press of business to do extra work, often working for several days in succession with very little sleep, the writer has been in the habit for many years of limiting the amount of food taken to not more than half the usual allowance, and sometimes to even a less quantity. The result has invariably been all that could be desired; since, although 
several pounds of flesh are often lost during an ordeal of this kind, when it is passed, and the usual routine of work is resumed, the digestive powers are intact, and able to digest the amount of food necessary for recuperation, so that a few days suffice to restore the usual weight, and without loss of either strength or time.

It is evident that the diet of each individual must be regulated in quantity according to his occupation. It must also be adapted to his age. A man engaged in severe physical labor, while he really requires less food, may be able to dispose of more food than one who labors with equal intensity in some mental pursuit. The body is wasted much more rapidly by vigorous brain labor than by physical exercise only. Indeed, it is asserted by our best authorities in physiology, that three hours of severe brain labor are equal in exhausting effects upon the system to ten hours of physical labor or muscular effort. It is evident, then, that a man who works his brain constantly for ten or twelve hours a day really needs more food to sustain his strength than a man who employs lis muscles for the same length of time.

But, as before remarked, the muscle laborer may be able to dispose of more food than the brain laborer, though he needs less, since his vital forces are not so completely exhausted by his work. In other words, the occupation of the muscle worker being less exhanstive than that of the brain worker, he can overeat with greater impunity than can the latter. Each should eat only the quantity actually required, if he would enjoy the maximum of health and vigor ; but for the man whose vital energies are daily exhausted by mental ef- 
fort, any excess in eating is certain to be most disastrous. We have examples of great literary men who have been large eaters ; but it is a noticeable fact that these persons, in many instances, while celebrated for their productions, often worked very leisurely, their fame being really more largely attributable to brilliant genins than to great application. In several cases, too, as in that of Charles Dickens, the hours spent in brain labor were chosen from the best of the day, much time being spent in physical exercise, by which means the integrity of the digestive organs was maintained to a degree that would otherwise have been impossible. In not a few instances, too, those great literary men who were noted eaters died early, their physical stamina being exhausted by the double drafts made upon it. Newton, when engaged in the most severe portion of his wonderful labor's in demonstrating the law of gravitation by computations respecting the orbit of the moon, confined himself to a spare diet of bread and water. The greatest amount of food is required by the person who is actively engaged in both physical and mental labor.

The amount of food required by an individual, as already intimated, varies at different periods of life, according to the degree of vital activity. In infancy and childhood, when the vital activities are at their highest degree of intensity, - when growth and development are to be maintained in addition to supporting the wastes of the system, - the demand for food is greater in proportion to the size of the individual than at any subsequent time. In adult life, when waste and repair are about equally balanced, a sufficient amount is needed to make good the daily loss from the various mental, 
physical, and other vital activities which can only be supported at the expense of tissue. Any larger quantity than this is excess.

In old age, when the assimilative powers are weakened by declining years, the amount of food which can be assimilated by the individual is even somewhat less than what is really needed; hence, as age advances, the quantity of food should be gradually diminished. Very many old people break down much sooner than they would otherwise do, were they more careful in this regard. When they lay aside their vigorous, active life, they should also curtail the quantity of their food. By this act of temperance, they might preserve intact to a much later period the integrity of their digestive organs, and so add years to their lives.

In not a few instances, the foundation of dyspepsia is laid by some mechanical injury, as a sprained ankle, a broken limb, or a severe bruise or cut, which reqnires rest from active exercise for a few weeks. Not considering the fact that much less food is demanded when a person is not engaged in active labor of any kind than at other times, the individual continues to eat heartily, and soon finds his digestive organs refusing to do their work from sheer exhaustion. On this account, it should be made a uniform custom to eat lightly on the weekly rest-day. The hearty Sabbath dinners in which many people indulge, making the day an occasion of feasting rather than a rest-day, cannot be too much condemned. The custom is without doubt responsible for many other forms of Sabbath-breaking, as no one can have clear perceptions of right and a quick sense of wrong when laboring under the incubus of an over- 
loaded stomach. For the hearty meal usually taken, it would be well to substitute a light one, consisting mostly of fruits and grains.

This plan, if pursued, would do away with much of the drowsiness in church of which many people and not a few pastors have abundant reason to complain. The intellect would be clearer, and hence better able to appreciate the privileges and comforts of religion. The sooner people recognize the fact that stomachs have much to do with religion, and that true religion includes the government of the appetite, and frowns upon abuse of the stomach as well as abuse of a fellow-man, the better it will be for both stomachs and religion.

Each individual must, to a considerable extent, be his own guide respecting the exact amount of food to be taken at a given meal. If the appetite has been so long abused that it is no longer a safe guide, then reason must rule. The individual should, at the beginning of the meal, determine just how much he will eat; and when the specified quantity is taken, he must resolutely stop eating, leaving the table, if necessary, to escape temptation. The practice of serving fruit, puddings, nuts, confectionery, and tidbits of various kinds as a dessert, is a pernicious one. In the first place, it is an inducement to overeating, since it is quite probable that enough has been eaten before the dessert is served. If the articles offered are wholesome, they should be served and eaten with the meal, as a part of it, and not at its close, in addition to the meal. Furthermore, it is generally the case that most of the articles served at dessert are wholly unfit to be eaten at any time, and so should be discarded. Dessert is really an in- 
genious device to lead people to make dyspeptics of - themselves by eating more than they need.

A man who desires to be at peace with his stomach should learn to stop when he has enough, no matter how strongly he may be tempted to do otherwise. There is much more truth than poetry in the old Scandinavian proverb, "Oxen know when to go home from grazing; but a fool never knows his stomach's measure." But experience, a dear school, onght after a time to teach the most unobservant person the amount of food lis stomach will bear without discomfort and without injury. If a person in fair health finds that after eating of wholesome food he is troubled with fulness of the stomach, dulness over the eyes sour stomach, eructations, or flatulence, he may be very sure that he is eating too much, and he should continue to diminish the amount taken at each meal until the symptoms mentioned disappear.

It is well to bear in mind that the danger is pretty much all on the side of overeating, the liability of eating too little-being very small indeed. The tendency to overeat will be greatly lessened by eating very slowly, masticating the food thoroughly, and eating only the simplest articles. One who has never made the experiment will be astonished to see how little food is really required to support life. The writer has lived for months at a time on an average of seventeen ounces of solid food per day, gaining flesh the whole time. Cornaro, an Italian nobleman, lived for many years on twelve ounces of solid food per day (by solid food is meant the weight ).

Numerous experiments by Letheby, Parkes, and 
many other scientists, together with a careful study of the dietaries of various classes of artisans, laborers, professional men, etc., show that life can be well sup. ported upon twenty ounces of carbonaceous and two and one-half ounces of nitrogenous food per day. Pugilists in training nsually take but twenty ounces of solid food, and numerous classes of individuals subsist upon a considerably less quantity.

By reference to the table of nntritive values given on pages 41,42 , it will be easily possible to ascertain the amount of nutriment consumed in any given quantity of different varieties of food. ' It is perhaps worthy of remark that the grains, as shown in the above table, are by far the most nutritions of all the various classes of food. It will be observed, for instance, that oatmeal, Indian meal, and peas contain three times as much real nutriment as lean beef. When economy must be considered in the selection of food, this is a very important consideration; and it becomes doubly evident when we consider that it takes eleven pounds of vegetable food, inclnding Indian meal, dry hay, ètc., to make one of beef. It thus appears that a pound of beefsteak, or second-hand grain, costs thirty times as much as a.pound of grain taken at first hand, besides being vastly inferior in quality.

\section{Deficiency in Necessary Food Elements.-} The food may be abundant in quantity, and yet deficient in one or more of the varions elements which go to make up true food. If the food is deficient in farinaceous and fatty elements, the individual will soon show signs of emaciation in consequence. A lack of the nitrogenous elements will occasion still more 
marked effects, cansing the stomach to lose its tone and vigor, thus giving rise to acidity, flatulence, and various associated disturbances. The deficiency of the coarser, innutritious elements of the food is also very soon felt by diminished activity of the stomach and bowels, both in secretion and in muscular action. Hence the great importance of choosing carefully and judiciously the articles of food to be taken, especially when a regular dietary is to be followed. Such a selection should be made as will supply to the system all the elements of mutrition in proper quantity. To employ a dietary in which any. one of the nutritive elements is deficient, although the quantity of the food may be all that the digestive organs can digest, is as really starration, and will as certainly occasion the same results ultimately, as total deprivation of food. To attempt to live on white bread and strong tea or coffee, is as certain to impoverish the blood as to refrain from eating altogether, the only difference being in the length of time required to bring about the result. Thousands of pale-faced, anemic, thin-blooded, nerveless, dyspeptic women owe all their troubles to an impoverished diet. Tea drunkenness is not an uncommon thing ; and in consequence of its pernicious inflnence, the sagacious physician not infrequently finds as wellmarked cases of scurvy among ladies of the higher classes of society as among the poorly fed sailors of the whaling vessel after a long voyage, with prolonged confinement to a monotonous saline diet. Young ladies who attempt to exist with little other food than tea, pastry, and' confectionery, need not wonder that they grow to be lank, sallow, hollow-eyed dyspeptics. Un- 
der such a regimen the most hardy quadruped would succumb.

Many parents weaken the digestive organs of their little ones for life by feeding them when very young upon such insufficient diet as corn-starch or arrow-root gruel, and similar preparations, and when they become older, upon fine-flour bread. Repeated experiments have shown that a dog will die of starration in a month when fed upon white or fine-flour bread alone. Fed upon bread made of the whole grain, or graham bread, dogs, as well as other animals, suffer no deterioration in weight or in strength. The difference between fine flour and graham flour is largely in the proportion of gluten which they contain. Fine flour is made from the innermost portion of the grain, which is almost pure starch, thus excluding the brain-, nerve-, and muscle-nourishing elements which are found chiefly in the portions of the kernel which lie next to the outer husk. Whole-wheat flour also contains portions of imnutritious matter which, under most conditions, are advantageous, encouraging both secretion and muscnlar activity of the bowels, and thus preventing constipation, which is often a forerunner of more serions disease of the digestive organs. There are cases in which the coarser portions of the bran do harm by causing irritation; but these do not often occur.

It should be added that the more recently perfected processes employed in the manufacture of patent flour, afford a product which is both fine and nutritions, both the innermost portion of the kernel and the bran being discarded. But in connection with this, there is also a grade of flour made, known as "family flour," which 
consists only of the central portion of the grain. The remarks respecting fine flour apply especially to this product, and not to the best brands of patent flour. The so-called "gluten flours", which are extensively advertised, are for the most part inferior to first-class wholewheat flour, and are never in any way superior to it. Much that is sold under the name of graham flour consists of inferior family flour mixed with coarse bran. Snch gralıam flour is not to be recommended. The best graham flowr is that made by the old milling process, in which stones, instead of steel rollers, were used for grinding.

While it is necessary to have all the elements of the food in proper proportion, it is of first importance that the nitrogenons elements be sufficient in quantity, even if it should be necessary to make use of an excess of farinaceous foods to secure the proper amount, since it is of these elements that the vital portions of the body are formed. The following table shows the respective amounts of different articles of food required to furnish the requisite quantity of nitrogenous matter for one day :-

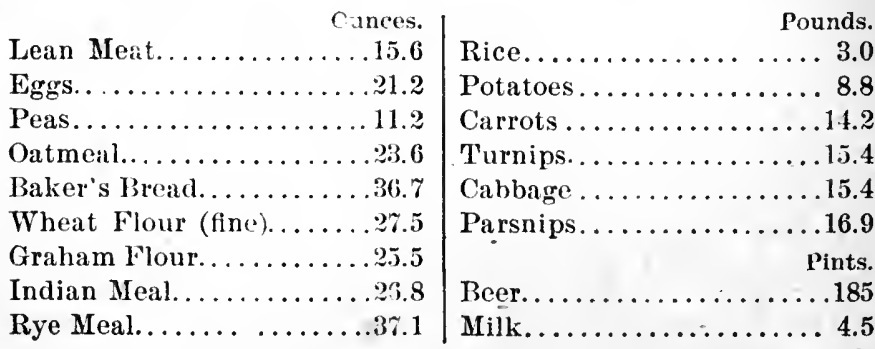

By reference to the above table, any one will be able so to combine various articles of food as to secure the 
proper amount of nitrogenous matter withont overloading the digestive organs, and yet give to the food the bulk necessary for good digestion. Evidently, it would overtax the stomach to digest turnips in sufficient quantities to supply the wants of the body, while lean meat would afford an insufficient amoint of bulk, as well as a deficiency of carbonaceous matter. By a combination of such nitrogenous seeds aś lentils, peas, and beans, or of oatmeal or wheat meal, with potatoes or other vegetables, the difficulty may be overcome, as also by combining eggs with the carbonaceons grains and vegetables.

The proportion of carbonaceous and nitrogenous food elements required for the maintenance of health is about one part of the latter to seven or eight of the former. The following table, inclnding a few of the more important articles of food, will give a clear idea of the relative proportion of the two elements in the articles mentioned :-

\begin{tabular}{|c|c|c|c|}
\hline $\begin{array}{l}\text { Album. or } \\
\text { Nitrog. }\end{array}$ & $\begin{array}{l}\text { Carbon- } \\
\text { aceous. }\end{array}$ & $\begin{array}{l}\text { Album. or } \\
\text { Nitrog. }\end{array}$ & $\begin{array}{c}\text { Carbon- } \\
\text { aceous. }\end{array}$ \\
\hline Lean Beef....... 1 & .5 & Wheat Meal or & \\
\hline Eggs. .......... & ].9) & Bread....... & \\
\hline Peas........... 1 & 2.7 & Rye Meal..... & \\
\hline Milk ..... & 3.6 & Potatoes... & 10 \\
\hline Fat Beef... & 5.0 & Carrots..... & 11 \\
\hline Oatmeal ....... 1 & 6.1 & Barley Meal..... . 1 & 12.7 \\
\hline IndianMeal ...... I & 7.7 & Rice......... 1 & \\
\hline
\end{tabular}

According to M. Germain Sée, of Paris, one of the most eminent French physicians, observations npon different races and physiological experiments show that the amount of albumen heretofore supposed necessary for the maintenance of health is greater than is actually required, and that the proportion of this element need not exceed one ninth to one tenth. About the same 
amount of fat is required, the balance to be starch or sngar. According to this authority, twenty to twenty-one ounces of water-free food is required for a daily ration, of which two to two and one-third ounces should be albumen or its equivalent, two ounces of fat, and the remainder starch and sugar. As we have elsewhere shown, starch is much to be preferred to sugar as a form of carbonaceous food. This remark, of course, applies especially to cane-sugar, since the sugar of fruits is already advanced in the digestive process, whereas cane-sugar hinders digestion, and produces gastric eatarrh.

The Quality of Food.-Man, like other animals, is made of what he eats, and we may well credit the assertion of an eminent author, that the general tendency of thought in any nation may be determined by the character of the national diet. True as this principle is when applied to the body in general, it is especially true in reference to the stomach. No organ is so directly and so profoundly affected by the quality of the food as is the stomach. Hence we may well consider with care the varions ways in which the digestive organs may become impaired through defects in the quality of the food.

Bad Cookery.-As a potent cause of dyspepsia, bad cookery deserves first mention in this connection. The real object of cooking is to render the elements of food more digestible. It is intended, indeed, to be a sort of partial preliminary digestion of the food; but the numerous devices of cooks and caterers, with their complex and indigestible mixtures, have so far subverted the original design of the process as to render 
cooking a means of making food indigestible as often as otherwise. Altogether too little attention is paid to the subject of cookery as a science. In the majority of cases the task of preparing food for the palate (the stomach is seldom thought of ) is intrusted to ignorant servant-girls or colored cooks, who compound their mixtures by "the rule of thumb," and without any reference whatever to the physiological wants of the body. Some slight indications of reform in this direction are shown in the establishment of schools of cookery in the larger cities, and lectureships on the subject in some of our female seminaries. To become a good cook requires as much native ability and far more practical experience than to become a musician or a schoolteacher, or even to enter some of the learned professions. The position of cook ought to be made so respectable and lucrative that it will attract persons of sufficient mental capacity and culture to make the art subservient to the purposes for which it was first employed and designed. A bad cook in a family is a worse enemy to the health, the comfort, and even the morals of the household, than would be a swamp generating malaria a half-mile away, a cesspool fever-nest at the back door, smallpox across the street, or a Chinese joss-house in the next block. Give us good cooks, - intelligent cooks, cooks who are thoroughly educated, - and the cure of nine tenths of all the dyspeptics may be guaranteed, without money and without medical advice.

Fried Food.- Of all dietetic abominations for which bad cookery is responsible, fried dishes are the most pernicious, Meats, fried, frricasseed, or other- 
wise cooked in fat, fried bread, fried vegetables, doughnuts, griddie-cakes, and all other similar combinations of melted fat with other elements of food, are most difficult articles of digestion. None but the most stalwart stomach can master such indigestibles. The gastric juice has little more action upon fats than has water. Hence a portion of meat or other food saturated with fat is as completely protected from the action of the gastrie juice as is a foot within a well-oiled boot from the snow and water outside. It is marvelous indeed that any stomach, under any circumstances, can digest such food, and it is far from remarkable that many stomachs after a time rebel.

It is principally for this same reason that rich cake, shortened pie-crust, and pastry in general, as well as warm bread and butter, so notoriously disagree with weak stomachs, and are the efficient cause in producing disease of the digestive organs. The digestion of the food being interfered with by its covering of fat, fermentation takes place. The clianges occasioned in the fat develop in the stomach extremely irritating and injurious aeids, which irritate the mucous membrane of the stomach, eausing congestion, and sometimes chronic inflammation.

Uncooked Food.-Raw foods and food which has been insufficiently cooked, are a frequent cause of indigestion. This is especially true of uncooked vegetables. Man is naturally a frugivorous animal, and is able to make use of vegetables and many grains as food only by the aid of cookery.

As will be recalled, the digestion of starch is effected by both the saliva and the pancreatic juice. Salivary 
d:gestion takes place in the month and the stomach,chiefly in the stomach, of course, as the food is not retained in the mouth for a sufficient length of time to allow the digestive process to proceed beyond the preliminary stage. In the digestion of starch, the starch is first rendered soluble, and is then converted into dextrin, and finally into malt-sugar, or maltose. The digestive principle of the saliva is incapable of acting upon raw starch, but the pancreatic juice digests raw starch, although with less facility than cooked starch.

By the action of heat the starch granules, which consist of the starch proper enclosed in little capsules, are ruptured, and thus the digestive juices can readily come in contact with and digest the starch.

By the prolonged action of moderate heat, or by exposure to a high temperature for a shorter time, starch is converted into dextrin. Similar changes take place in ripening fruit. Green fruit contains a large amount of starch ; in the process of ripening, this starch is converted into dextrin and sugar, as in the process of digestion, so that in the use of fruit the starch is taken in a partially or completely digested state, which accounts for the easy digestibility of this kind of food.

When starchy substances are eaten raw, the saliva being unable to act upon the uncooked starch, the gastric juice cannot gain access to the albuminous substances present, and hence these particles are not broken up, and being retained in the stomach for too long a time, fermentation and irritation are the result. It is for this reason that green fruit and raw vegetables occasion so much disturbance of the stomach and 
bowels, these immature foods containing large quantities of starch irr a very indigestible state. By cooking; unripe fruit and vegetables may be in a great degree deprived of their injurions properties. Cooking is thus a sort of preliminary digestion.

In Scotland, the eating of oatmeal imperfectly cooked is a very common practice, the result of which is almost universal suffering from a peculiar form of indigestion due to it, known as water-brash. The Scotch farmer prefers his oatmeal raw because of its indigestibility, as the result of which it "stays by the stomach" longer than when well cooked. Nearly all kinds of food are much more easy of digestion after cooking than before, providing the cooking is performed in the proper manner. For vegetables and grains, cooking is especially necessary.

Decayed Food. - Mnch harm comes from eating food which has made appreciable advancement in the direction of decay. This is true both of vegetable and animal food. By the process of decomposition, poisonous elements are developed in animal and vegetable substances, especially the former, which do not naturally exist there. If decomposition is far advanced, these poisons may exist in such quantity as to produce immediate ill effects, sometimes occasioning death in a few hours. Instances of this kind have often occurred from eating canned meats which had spoiled, or which had been kept for a short time after opening. The practice in vogue in some other countries, and to a certain extent in this, of keeping meat for some days before eating, so as to give it tenderness and a "high" flavor, is a most pernicious one. Better far, for health, 
is the barbarous Abyssinian custom of eating the flesh raw and while still warm and quivering.

For dyspeptics such food is especially bad, since digestion is so slow that decomposition is not corrected, as it is to some extent in a healthy stomach, by the gastric juice, but is allowed to continue, with all its serious consequences Even if no immediate effects follow the use of such food, the poisons generated may be absorbed, and appear later in some form of blood-poisoning. The stomach of a hyena may be able to digest the putrid flesh of a decaying carcass; but man's stomach was not intended for scavenger use, and requires fresh, untainted food.

Shell-fish, and, in fact, fish of all kinds, are particularly prone to decomposition, undergoing this change much more quickly than do other forms of animal food. It is partly for this reason that poisoning from the use of oysters and fish occurs so frequently, and sometimes with fatal results. Experiments recently conducted by Trombetta, an eminent French physiologist, show that decomposition of flesh begins within twenty-four to forty-eight hours after the death of the animal, even when the flesh is kept within a refrigerator. Germs rapidly increase after the death of the animal, and even after so short a period are found in abundance. It thus appears that all flesh food must contain more or less of poisonons matters, the products of decomposition. Bonchard has shown that meat juice is very deally in its effects.when injected into the veins of an animal, in consequence of the poisons which it contains. Beef tea is, for the most part, marle up of soluble substances contained in the flesh of the animal at 
death, or developed by the action of germs after death. An eminent French physician, speaking of beef tea, remarked that "it is a true solution of ptomains" (poisons).

Cheese is another article which contains poisonous substances known as ptomains, in great quantities, and not only poisons, but germs capable of producing poisons in abundance in the stomach. Cases of cheese poisoning are a frequent and sometimes fatal result of the nse of cheese. Cholera morbus, a disease due to germs, is frequently induced by eating cheese. That poisoning does not always occur from the use of cheese is due to the fact that the gastric juice of a healthy stomach is capable of destroying a considerable quantity of germs, and that the liver is capable of destroying the poisons introduced with the cheese in considerable amount. Milk sometimes gives rise to indigestion and serious illness in consequence of the disease-producing microbes which it contains.

Soft Food.- The structure of man's teeth indicates that he was intended to employ a diet consisting of food of such consistency as to require vigorous mastication. His jaws are armed with thirty-two strong teeth, compactly arranged in the mouth in such a manner as to make them most available for use. Obeying the general law governing all organized struetures, by which organs develop or degenerate according as they are used or allowed to remain inactive, the teeth retain their health if vigorously employed in the mastication of solid food, but rapidly undergo decay when not thus used. We have an illustration of this in cows fed on distillery slops. The teeth of such 
animals decay and drop out for want of use, while those of cattle which keep their teeth actively employed in chewing the. cud, are preserved intact.

The effect of soft food upon the teeth of an animal is well shown in the accompanying illustrations.

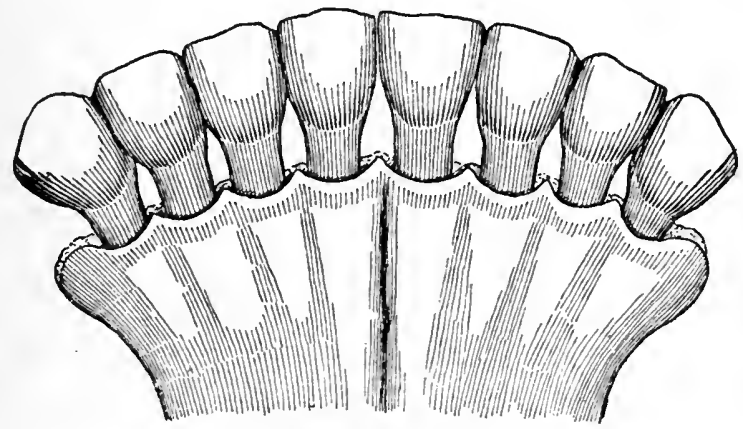

Fig. 14.- Teeth of Healthy Cow.

Fig. 14 represents the teeth of a cow fed upon proper food. Fig. 15 shows the teeth of a cow fed upon distillery slops.

The same is true of human beings. Eating soups, gruels, and other soft foods, to the exclusion of articles requiring mastication, ruins the teeth at the same time that it disorders the stomach through the taking of too much fluid, thus causing deficient insalivation.

Too Abundant Use of Fats.-Unfortunately for the poor stomach, the opinion prevails almost everywhere that food made rich with fat is the most nourishing. Undoubtedly fat is an element of nutrition, and can be digested and assimilated when taken in proper quantities and in a proper manner; but the excessive use of fats of various kinds, as lard, suet, butter, and other animal and vegetable fats or oils, is a 
prolific cause of certain forms of indigestion, especially that known as bilious dyspepsia. The famous physiologist Bernard many years ago determined by careful experiment the fact that the free use of fats greatly reduces the biliary secretion, the quantity of bile being diminished in some instances to a very small fraction of the amount secreted when only pure water or food containing little fat was taken. When it is remembered that the bile is an essential element for the digestion of fat, it will be seen that a diminution of this digestive fluid in connection with the taking of an extra quantity of oleaginous matter is a most unfortunate circumstance, since it is thus absent when most needed. This fact sufficiently well accounts for the distressing symptoms which accompany the excessive use of fats by those whose digestion has been already weakened by

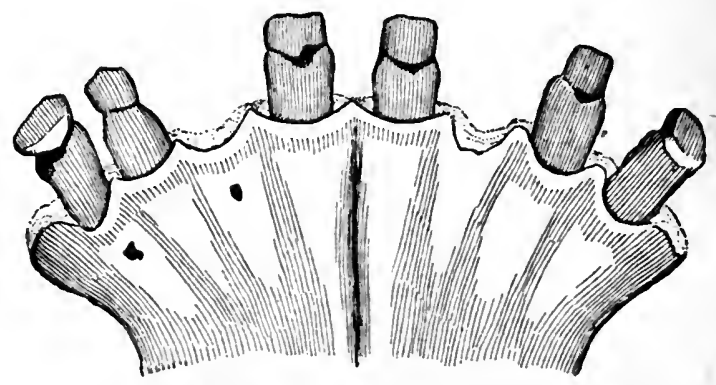

Fig. 15.-Teeth of Cow Fed on Distillery Slops.

abuse of this kind. The diminished quantity of bile produced by the liver is also sufficient cause for the condition established by the over-use of fats, vulgarly known by the expressive term "bilious." The elements which ought to be eliminated from the system 
are retained, clogging thé vital machinery, and giving rise to many symptoms of systemic poisoning.

When it is remembered that the bile is the antiseptic agent by which the contents of the small intestines are preserved from decomposition, it will readily be seen that a deficiency of bile must result in decomposition of food elements, and the formation of poisonous substances. The absorption of these substances still further disturbs the liver, contaminates the body, and produces the condition of general poisoning which is commonly termed biliousness or bilious dyspepsia, a state which is not to be remedied by the use of liver medicines, purgatives, etc., but by correction of the diet.

Fats readily undergo decomposition in the stomach, especially in a stomach which is dilated so that the food is too long retained, or in one which is the seat of gastric catarrh. It is on this account that fats, even in the form of butter or mingled with the food, as in rich gravies or the shortening in pie-crust, are so often a source of irritation and disturbance in dyspeptic stomachs.

The use of rich foods is not infrequently a cause of bilious headache or a bilious attack. Animal fats are more likely to undergo this decomposition than are vegetable fats. Ordinary butter is particularly unwholesome, for the reason that it always contains multitudes of microbes derived from the milk, which are rapidly developed in the stomach, producing decomposition of the fat, and thus forming irritating fatty acids. Only sterilized butter is fit for human consumption. 
Cooked fats are also much more irritating and likely to produce indigestion than uncooked fats. The process of cooking develops acrid fatty acids which are extremely irritating to the gastric mucous membrane. This is one of the reasons why fried and fricasseed foods, griddle-cakes, doughnuts, Saratoga chips, etc., are so harmful to digestion. Fats taken in the form of cream are generally more digestible than in any other way. In some cases, however, there is an inability to digest the casein of milk in the form of cream ; for such cases sterilized butter is to be preferred. The least harmful mode of using a free fat is in the form of sterilized butter taken with cold bread. Melted fat taken with farinaceons substances is extremely hurtful, as the starchy particles are so completely surrounded and permeated by the fat that the saliva, which should act upon the starch in the stomach, is unable to do so, thus leading to indigestion.

The use of natural foods containing a sufficient quantity of fat is much to be preferred to the use of - free fat. In cream the fat is in a finely divided state in which it can mingle readily with the fluids of the stomach ; hence it does not smear over those food substances which are acted upon by the gastric juice and the saliva, thus preventing their digestion. Neither the gastric juice nor the saliva has any action upon fat, and for this reason free fats may constitute a very serious obstacle to the action of both the saliva and the gastric juice.

Nuts also afford a most excellent form of fat. The popular idea that nuts are difficult of digestion is the natural outgrowth of the common habit of swallowing 
them without mastication. Nuts have a firm, hard structure, requiring very thorough mastication. If pains is taken to chew them very thoroughly, nuts are as digestible and wholesome as any other food, and they are highly nutritious. Lack of sound teeth is, in some cases, an almost insuperable obstacle to the proper mastication of nuts, as well as of other hard foods. The writer has succeeded in meeting this difficulty-in the case of nuts, at least-by preparing from some of the best and most natritions nuts a very fine and thoroughly cooked meal, and also a nut cream or butter. The latter, like cream, is nearly a perfect cmulsion, but is, at the same time, so rich in fat as to be an excellent substitute for butter. It is especially useful in seasoning foods, and serves a most excellent purpose as a dressing for vegetables and other foods which are deficient in fats. These nut preparations are manufactured and sold by the Sanitas Food Co., Battle Creek, Mich. (See page 241.)

The Use of Sugar in Excess.- While sugar, like fat, is a true alimentary principle, capable of aiding in the maintenance of life when employed with the other elements of food, if used in excess, it becomes a serious source of disease. When used alone, it is utterly incapable of supporting the vital activities of the body, being, in this respect, analogous to starch, its food equivalent. The popular idea that sugar nourishes the nerves or the brain, makes the teeth sound, and is both harmless and wholesome, is quite a mistake, as many an innocent little one whose fond parents shared in the general error, has found out to the regret and sorrow of his friends. 
The different forms of sugar, molasses, sirup, treacle, honey, etc., are essentially the same in their effects, except that molasses and honey sometimes contain peculiar elements that to some persons seem to be almost active poisons. This is especially true of honey.

The injury from the use of sugar or other saccharine substances is occasioned, first, by the readiness with which it undergoes fermentation when subjected to warnth and moisture. In the stomach it finds all the conditions necessary for inducing fermentation; and were it not that saccharine substances in solution are usually so quickly absorbed that it is difficult for the chemist even to detect their presence in the stomach, this change would always occur. When a larger quantity is taken than can be absorbed promptly, or when taken in such form as to make ready absorption impossible, as in the case of preserves and sweetmeats of various sorts, acid fermentation does, occur, and with serious results not only to the stomach, but to the whole system. The fermentation set up not only develops acids and gases from the sugar, but, being communieated to the other elements of the food, as the starch and especially the fatty elements, still worse forms of fermentation or decomposition occur; and the food is thus rendered unfit to nourish the body, while the mucous membrane of the stomach and intestines is irritated by the contact of unnatural corroding elements in the food, and through their absorption, the whole system becomes affected.

The excessice use of sugar also greatly overtaxes the liver, which has an important part to act in its digestion, interfering with the proper performance of 
its other functions, especially those of bile-making and poison-destroying, and thus leaving the elements which it ought to eliminate, to accumulate in the system. Thus a person may become "bilious" from the overuse of sugar as well as from the excessive use of fats.

When taken into the stomach in considerable quantities, sugar gives rise to a profuse flow of mucus, and may thus eventually develop chponic catarrh of the stomach, which, extending into the duodenum and the bile ducts, produces catarrhal jaundice and the partial closure of the biliary passages. When sugar is used to excess for a considerable length of time, the liver finally loses its ability to retain the sugar in its tissues in the form of glycogen,- one of its most important functions, - so that an excessive quantity of sugar is thrown into the blood, and, being eliminated by the kidneys, appears in the urine. This is tho most frequent and most common origin of diabetes. The writer has met cases in which nearly a pound of sugar was thus eliminated through the kidneys in twenty-four hours, and quite a number of cases in which more than half a pound of sugar was thus thrown ont of the body in the same length of time. Sugar is never fonnd in the urine in a state of health.

Excessive use of sugar is also a most common cause of obesity. The excess of carbonaceous material in the form of sugar being deposited as fat, or adipose tissue, overwhelms the heart so that this excess of fat is sometimes attended by fatal. results.

Excessive Use of Flesh Food.- The flesh of animals, especially lean meat, is usually considered the most easily digested and strengthening of all foods. 
This fact, together with the stimulating character of this class of foods, is, perhaps, the cause of its extensive use in English-speaking countries, especially in England and America. The fact that immediate distress and unpleasant symptoms relating to the stomach itself less frequently follow the use of flesh food than the use of many other foods, is perhaps one cause for this misapprehension. While it is true that meat, especially lean meat, is more readily digested in the stomach than coarse vegetables, and is less likely to ferment, forming gas and irritating acids in the stomach, than are saccharine and starch foods, it is nevertheless true that meats are, of all foods, most exciting and stimulating to the stomach; - and when freely used, the ultimate result is to produce a condition of debility in the stomach, and in some cases gastric catarrh and other stomach disorders may be attributed to the excessive use of meat. The worst effects, however, from an excessive use of meat are to be songht in the liver and in the body in general, resulting in rheumatism, gout, neurasthenia, and a great variety of diseases which have their origin in the introduction of an excessive quantity of toxic substances into the body through the use of meat.

The practice now much in rogue, of administering large quantities of raw or under-done meat, either in the form of an ordinary steak or chop, or specially prepared, as by scraping or mincing, has been productive of a vast deal of injury. The writer has noted several instances of acute Bright's disease in which general symptoms of poisoning and various nerrous maladies could be directly attributed to following, for a number of months, 
a prescription requiring raw meat as a principal article of diet.

Condiments. - By condiments are meant all substances added to food for the mere purpose of rendering it more palatable, but possessing no positive nutritive value in themselves. Mustard, vinegar, pepper, cinnamon, and various other spices are included in this category, together with salt, although the lastnamed article is by some held to be of the nature of a food, and supposed to supply some want in the body.

Mustard, pepper, pepper-sauce, cinnamon, cloves, cardamoms, and similar substances are of an irritating, stimulating character, and work a two-fold injury upon the stomach. By contact, they irritate the mucous membrane, causing congestion and diminished secretion of gastric juice, when taken in any but very small quantities. This fact was demonstrated by the observations of Dr. Beaumont upon St. Martin. After several years' careful study of the relations of various foods, drinks, etc., to thie stomach, Dr. Beaumont stated, in summing up his experiments, that "stimulating condiments are injurious to the healthy stomach." He often saw congestion prodnced in the mucous membrane of St. Martin's stomach by his eating food containing mustard, pepper, and similar condiments.

When taken in quantities so small as to occasion no considerable irritation of the mucous membrane, condiments may still work injury by their stimulating effects, when long continued. The stomach being at first excited to more than natural activity, afterward suffers from reaction, and is left in an inert, diseased state, incapable of secreting sufficient gastric juice to supply 
the needs of the system in digesting food. This final result is often averted for some time by increasing tha quantity of the artificial stimulus; but nature gives way at last, and chronic disease is the result.

In experiments conducted in the Laboratory of Hygiene connected with the Battle Creek (Michigan, U. S. A.) Sanitarium, we have found that the use of condiments does not increase the flow of either saliva or gastric juice, but causes an ontpouring of a great quantity of protectire mucus. When this becomes habitual, the individual is the subject of gastric catarrh, a disorder which is by no means easily cured. The writer quite agrees with the conclusions of the small boy who, having tasted horseradish for the first time, spat it out, remarking to his mother, "Mamma, I think I won't eat that till it gets cold." Substances which are hot when they are cold are not fit to be eaten. The irritating and astringent properties of certain regetable substances were doubtless put into them as warnings against their use by human beings.

In Mexico, where pepper, mustard, and other condiments are probably used more freely than in any other part of the world, gastric catarrh is an almost universal disease.

In the case of salt, there are several objections to be urged, which are at least cogent against its excessive use; and by excessive use is meant a quantity which causes thirst either at or after meals, oceasioned by the fererish state of the stomach induced by the caustic properties of the saline element. According to Debove, physiological experiments have shown that salt, when taken in considerable quantities, interferes with the digestion of albumen. This statement agrees with 
the writer's own laboratory experiments, as well as with the experience of sailors, who, when making free use of salt meat, suffer from scurvy and a long list of symptoms indicating malnutrition and tissue starvation. Experimental evidence shows that human beings, as well as animals of all classes, live and thrive as well without salt as with it, other conditions being equally favorable: This statement is made with a full knowledge of counter arguments and experiments, but not without abundant testimony to support the position taken.

The author does not, except in rare instances, advise the entire discontinuance of the use of salt ; nevertheless, he believes that it may be greatly reduced in. quantity by all who use it, without detriment, and with real benefit. Leriche and others have shown that salt, even in so small a proportion as one per cent., diminishes both the amount and the efficiency of the hydrochloric acid of the gastric juice, and thus gives rise to fermentation, one of the most common symptoms of indigestion, and a cause of many other morbid conditions.

Salted food is very hard of digestion; and when it is taken for a long time, the stomach often fails. A piece of fresh fish which will digest well in one hour and a half, requires four hours after salting, according to Dr. Beaumont.

Pickles.-Cucumbers, peaches, green tomatoes, and numerous other fruits and regetables are sometimes preserved by saturation with strong vinegar. Sometimes whisky or some other alcoholic liquor is added to increase the preservative property of the vinegar; but the same process which makes it impossible for the fruit or regetable to ferment or decay, makes its digestion 
equally difficult, if taken as food. Pickles are exceedingly unwholesome as an article of diet, and are often the cause of acute dyspepsia. Those addicted to the free use of pickles may be assured that they must certainly part with their favorite dainty or bid farewell to good digestion. Cucumbers preserved with salt or vinegar are next to impossible of digestion. The proverbial unhealthfulness of this vegetable is a popular notion based on experience with the article prepared with rinegar and salt. These chemical agents harden the delicate structures of the vegetable, and render it almost unapproachable by the digestive juices. The pure vegetable, unsophisticated by condiments, is no more harmful than other green vegetables.

Vinegar.-As the use of vinegar is continually increasing, attention should be called to the fact that it may be a cause of disease. Ordinary vinegar contains about five per cent. of acetic acid, its principal ingredient. Like alcoholic liquors, vinegar is a product of fermentation, being the result of carrying a little farther the same process by which alcohol is produced. Vinegar is much more irritating to the digestive organs than an alcoholic liquor of the same strength. Its exciting nature makes it extremely debilitating to the stomach. Dr. William Roberts, of England, has shown that so small a proportion of vinegar as one per cent., completely arrests the action of the saliva upon starch. The writer's own experiments have confirmed the observations of Dr. Roberts.

The moderate use of a light wine or of ale or beer is much less destructive to the digestive organs than a free use of vinegar. This remark is made, not to com- 
mend wine or beer, however, since these substances are possessed of no virtue, and are capable of doing a vast deal of harm. There is really no need of resorting to so inferior a source for a mild acid, as we have the want met most perfectly in lemons, limes, citrons, and other acid fruits. As a dressing for some kinds of vegetable foods, lemon juice is a perfect substitute for vinegar. Recent observations have shown that the vinegar eels which are nearly always to be found in "good cider vinegar," often take up their abode in the alimentary canal, becoming intestinal parasites, and producing much mischief. (Fig. 16.)

Vinegar is often adulterated, containing a very small proportion, if any at all, of real applejuice, its acidity being due to hydrochloric or sulphuric acid; therefore such vinegar is even

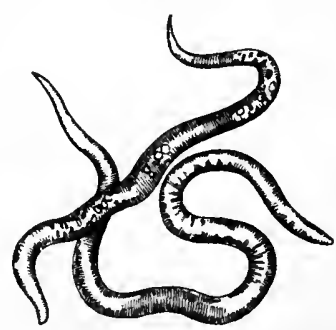

Fig. 16.-Vinegar Eels. more destructive to the functions of the stomach and also to the teeth than ordinary vinegar.

Tea and Coffee.- Classing these favorite beverages with causes of dyspepsia will certainly call forth - a loud protest from the numerous devotees of " the fragrant eup," as among the number of those who argue for their use are numerous learned professors, as well as nearly the whole sisterhood of the maidens, wives, mothers, and grandmothers of the nation, along with a good proportion of the brothers, husbands, fathers, and grandfathers also. Nevertheless, it can be easily shown that whatever action may be assigned to these beverages, it is unfavorable to digestion, rather than 
otherwise. Leaving out of consideration the objections which may be urged against the use of tea and coffee on other grounds, the following may be offered as reasons why they are objectionable on account of exerting an injurious influence upon the digestive organs :-

1. Both tea and coffee contain an element resembling tannin, which precipitates or neutralizes the pepsin of the gastric juice, and so weakens its digestive power.

2. Thein and caffein, the active principles of tea and coffee, are toxic elements which diminish the activity of the glands of the stomach by which the gastric juice is formed, thus interfering with the digestion of albumen and other proteid substances.

3. Both tea and coffee are objectionable on the same ground as other beverages in connection with meals, on account of their disturbing the digestion by dilution and consequent weakening of the gastric juice, and by overtaxing the absorbents, thus delaying the digestion of the food, and giving rise to fermentation.

4. Experiments made by Dr. William Roberts, and repeated by the writer, show that tea, even in a comparatively small amount, destroys the starch-digesting properties of the saliva, - another fact which accounts for the universal existence of indigestion in tea and coffee drinkers.

Alcohol.- We have not space in this connection to dwell at length upon the damaging effects of alcohol upon the human system, nor the full details of its effects upon the stomach. The following facts, however, are well worth the consideration of those who believe 
in the use of alcohol either moderately or with greater freedom :-

1. Alcohol itself is an active poison, which when received into the stomach in a concentrated state, is almost as quickly fatal to life as is prussic acid or strychnia. It precipitates the pepsin of the gastric juice, rendering it inert.

2. It irritates the gastric mucous membrane when taken in any but extremely small quantities, even beer and the weaker liquors having this effect when long continued.

3. The ultimate effect of alcohol is to cause degeneration of the secreting glands of the stomach, by which its utility as a digesting organ is destroyed.

Sir William Roberts, the eminent English physician whose authority we have already quoted, has shown that alcohol diminishes the activity of gastric digestion. In experiments made by the writer in the Laboratory of Hygiene (Battle Creek, Mich., U. S. A.) it has been found that half a pint of light wine lessens the digestive activity more than one half; and that an ounce of alcohol, well diluted, almost completely arrests stomach digestion.

Dr. Beaumont's observations on the effects of alcohol are very positive and distinct in their indications. St. Martin being an intemperate man, oceasionally indulging frecly in drink, Dr. Beaumont had an opportunity of observing the effects of its use, as by the aid of a strong light he could look directly into his patient's stomach through the window provided by the remarkable accident from which he had suffered. After he had 
been drinking freely for several days, Dr. Beaumont found the mucous membrane exhibiting inflamed and ulcerous patches, and the secretions very greatly vitiated, the gastric juice being diminished in quantity, viscid, and unhealthy, although St. Martin vlid not complain of any unusual feelings, and his appetite being apparently unimpaired. The condition continned to become still more aggravated for a day or two, when the doctor found, to use his own words, that "the inner membrane of the stomach was exceedingly morbid, the erythematic appearance more extensive, and the spots still more livid. From the surface of some of them exuded small drops of grumons blood; the aphthous patches were large and very numerous, the mncous covering thicker than common, and the gastric secretions very greatly vitiated. The gastric fluids extracted were mixed with a large proportion of thick, ropy mucus, and a considerable muco-purulent discharge slightly tinged with blood, resembling the discharge from the bowels in some cases of dysentery."

It will be remarked that notwithstanding the very serious condition of his stomach, St. Martin was unconscious of any great disturbance there. This was partly due, no doubt, to the paralyzing effect of alcohol upon the nerves of sensibility. It is owing to this fact that so many suppose that alcoholic drinks have no specially bad influence upon the stomach, when really their stomachs are well-nigh useless from disease, but too insensitive to indicate their condition.

The popular idea that beer, ale, and other similar liquors are very nourishing, has been shown to be an error: Alcohol is in no sense a food. Professor Lie- 
big, the eminent German chemist, has shown by careful analysis that the amount of nutriment contained in a whole hogshead of the best German beer is less than that contained in one ordinary loaf of bread.

Tobacco.- Not infrequently, though less often than is the caso with alcoholic liquors, this narcotic drug is recommended as a remedy for dyspepsia. Nevertheless, in the case of tobacco, as in that of alcohol, the remedy suggested is itself an active cause of stomach disease. Only on the similia similibus plan conld either one be reasonably employed. Both smoking and chewing weaken and debilitate the digestive organs, though both of these practices are thought by those who indulge in them, to stimulate the process of digestion. This it probably does for the time being, but only at the expense of subsequent injury. Snuff-taking, especially, prodnces gastric irritability, probably by reflex sympathy of the mncous membrane of the stomach with that of the nasal cavity, which is irritated by the direct contact of the acrid drug.

The ilea that tobacco is beneficial because of its supposed antiseptic properties, in that it may aid digestion by disinfecting the month, is without foundation, since the teeth of tobacco users are no less. prone to decay than those of other people, and cancer of the mouth, one of the most fatal and incurable of germ diseases, is almost exclusively confined to tobacco users. Tobacco users are also no less subject to pnemmonia, consumption, and other maladies, the germs of which enter the body through the mouth and nose, than are other people.

The immense waste of saliva occasioned by chewing 
and smoking may fairly be considered as one of the means by which the system sustains loss and injury through the use of tobacco. An eminent physiologist reported the loss of several pounds in a week, as the result of the stimulation of the flow of saliva by constant mastication for experimental purposes. This explains the loss of flesh in cases of tobacco using; and there is an equiralent loss of strength. It is apparent that the loss of flesh is also in part attributable to the physical damage of the body by the poisonous nicotine, - certainly a very doubtful sort of remedy.

Those who chew or smoke to prevent excess of fat, should understand that any drug which will exert such an influence upon the system must be a powerfully destructive agent. Those who succeed in keeping down fat by the use of tobacco may depend upon it that they are doing so only at the ruinous expense of their digestive organs, and may look forward with certainty to the breaking down of the nervous system.

Hard Water.- So little attention has been paid to this really common cause of indigestion by writers on this subject, that we cannot forbear mentioning it here. Experience has often proved that the use of hard water impairs the integrity of the stomach sooner or later, when long continued; and in numerous instances its effects are almost immediate upon persons who visit a hard-water district, having been accustomed to the use of soft water. The cause of these injurious effects is undoubtedly attributable to the lime and magnesia which are contained in water called hard. These alkalies, as already seen in considering the physiology of digestion, neutralize the gastric juice, and thus work 
mischief. There is little necessity for the use of hard water in any part of this country. Where there are not soft-water wells or springs, rain-water may be caught and preserved in cisterns, and by boiling and filtration through carbon filters, made pure and palatable for drinking and cooking purposes. Boiling hard water greatly diminishes its hardness by precipitating the lime which it contains. There is no foundation for the theory that hard water is in any respect more excellent for use than pure soft water. Distillation is the most efficient of all means of securing pure water. A very convenient and inexpensice apparatus for distillation is manufactured by the Modern Medicine Co., Battle Creek, Mich.

Alkalies.-For the same reason, soda, saleratus, and the numerous compounds of these substances with ammonia, alum, cream of tartar, etc., are all objectionable on the same grounds as hard water. Being alkaline, they antagonize the action of the acid gastric juice, and thus weaken digestion. There is no more active dyspepsia-producing agent than soda or saleratus biseuit, one of the most common articles of food to be found on the tea-tables of rich and poor in this country. Doubtless well-prepared baking-powders are much preferable to soda and cream of tartar or saleratus and sour milk, mixed by the cook in accordance with the not remarkably accurate "rule of thumb," through which bungling chemistry the biscuit often present a golden hue, which may be quite attractive to the eye, but gives to the tongue quite too distinct a flavor of soda and potash to be agreeable to a fastidious taste, to say nothing of the probable effect upon a stomach not impregnable to the attacks of chemical agents. In baking-powders, 
the various ingredients are so mixed as to leave nearly neutral products, and yet these compounds are scarcely less permicious in their influence upon digestion than the original chemicals from which they are formed.

The constitnents of the best baking-powders, when mixed with water, are essentially the same as those of Rochelle salts. Many baking-powders contain alum, and nearly all contain more or less ammonia. Both of these substances have been shown to be extremely detrimental to the digestive organs. Careful ehemical tests made by the writer and by others, have shown that anmonia, when used as a raising agent, is driven off by the heat only to a small extent, a sufficient amount remaining to occasion great damage to the digestive functions.

Alkalies of all kinds are injurious to digestion because of their neutralizing the gastric juice. Hypopepsia and apepsia result from a long-continued use of alkaline substances in the food or for the relief of sour stomach, aeidity, etc.

Perverted Appetites. - Strangely perverted tastes, as shown in a fondness for earthy and other inorganic or innutritious substances, while sometimes the result of dyspepsia, are often the cause of stomach disorders. They are either the result of nervous or mental disease, or are adopted as a habit through example. In South America there are whole tribes of human beings who habitually eat considerable quantities of a peculiar kind of clay. Several North Amerieun tribes have the same habit, being known as clay-eaters. A similar propensity sometimes appears among more civilized human beings, being almost exclusively confined, how- 
ever, to young women, chiefly schoolgirls, who acquire the habit of chewing up slate pencils, and gradually become so fond of such eartly substances that they have in some instances been known to eat very considerable quantities of chalk, clay, and similar substances. While indicating a lepraved state of the system, and often of the mind also, this practice has a very pernicious effect upon the stomach, which is not intended, as is that of the fowl, to receive inorganic matter of that sort.

The amount of abuse of this sort which the stomach will stand, however, is quite astonishing. Dr. Pavy tells a story of an American sailor who saw a juggler pretending to swallow pocket-knives With the characteristic recklessness of a sailor, and supposing that the knives were really swallowed, he attempted to do the same thing himself, and succeeded in getting down four. Three of these were passed off in a day or two, but he never saw the other. Six years after, he swallowed fourteen knives in two days, and was taken to a hospital, where "he got safely delivered of his cargo." He was not so fortunate on a subsequent occasion, when he paid dearly for his folly, lingering in misery for some time until he died, when his stomach was found to contain a number of rusty knife-handles, blades, springs, etc., the organ being greatly contracted and corrugated in consequence of the violence which had been done it. As a general rule, the innutritious parts of foods, as the skins of fruits and regetables, the seeds and cores of apples and similar fruits, should be carefully separated from the nutrient portions, and discarded. 
Adulterations of Food.- The numerous adulterations of food which are now so extensively practiced must be recognized as a not unimportant canse of funetional disease of the stomach. Alum in bread and in baking-powders; lead in drinking-water which has passed through lead water-pipes, or has been stored in lead eisterns, or collected from a roof covered with sheet-tin containing lead; lead occurring in the tin cans used for preserving fruit, or in tin pans or other tinned ware, or in the glazing of kettles; vinegar containing sulphurie and other strong mineral acids ; pickles boiled in copper or brass vessels, and thus poisoned with copper ; sugar made from corn, refuse starch, etc., and containing iron, sulphuric acid, tin, ete.; flavoring extracts made by purely chemical processes, and containing not a drop of the extract of the fruit after which they are named,- these, with numerous other equally harmful adulterations, may be reckoned among the active causes of indigestion.

Unseasonable Diet. - The failure to recognize the necessity of adapting the diet to the season and climate is a prolific source of a certain class of dyspeptic disorders. This is especially noticeable when the use of large quantities of carbonaceous food, especially fats and sugar, which may be used in the winter with comparative impunity, is continned into the warn season of the year; or when a diet of this sort is continued in a warm climate by persons who have been accustomed to it in a cold country. It is this sort of transgression of the laws of digestion that gives rise to "spring biliousness," "bilious dyspepsia," ete., in many persons. Large quantities of fat and sugar are not well tolerated 
by the stomach at any time; and in warm climates, and in the warm season of eold and temperate latitudes, they are exeeedingly injurious.

Pressure upon the Stomach.- The stomach is remarkably sensitive to-pressure. It even sometimes becomes temporarily paralyzed by exeess in eating, or by the aceumulation of gas from fermentation, by the distention of its walls. It is equally liable to injury of a similar sort from external canses. A sudden blow upon the stomach has been known to produce almost instant death, through the impression made upon the sympathetic nerrous system.

The wearing of eorsets, and tight-lacing with or without the eorset, are eommon causes of dyspepsia, as well as of other serious diseases. Wearing the pantaloons drawn tight and withont suspenders has a similar effect upon men. The soldiers of the Russian army once suffered so mueh from this eanse that it became neeessary to correct the evil by a royal edict for the purpose. Very soon after the evil practice was diseontinued, the effects disappeared. Bookkeepers and school-children, from sitting at a desk; seamstresses and tailors, from stooping over at their work; shoemakers, weavers, and washerwomen, from direct pressure upon the stomach incidental to their work, are apt to suffer from disturbance of that organ.

The disturbances produced by compression of the stomach, constriction of the waist, or bad positions in sitting are chiefly due to displacement of the stomach, resulting in too long retention of food, thus giving excellent opportmity for the development of germs taken in with the food, together with the irritating 
acids and poisonous substances which result from their growth.

Brain Work. - Mental labor, if agreeable and pleasant, is a most healthful occupation. There is no evidence for believing that brain work of that sort ever disagreed with the stomach or impaired its functions in any degree ; but mental worry, discontent, anxiety, and gloom are most unfarorable conditions for digestion, and under their influence few stomachs can long maintain their integrity.

Lack of Exercise.- Idleness is one of the most unhealthful of conditions. A lazy man is never well. Exercise aids digestion by increasing the activity of the diaphragm and the chest, as has been previously explained. It is also useful by purifying the blood and aiding the vigor of the circulation. The detrimental influence of idleness or a sedentary life upon digestion. is very clearly shown by the results of such a life upon the appetite. In a normal state the appetite is a good index to the condition of the digestive organs, - in other words, a good appetite means good digestion; loss of appetite means loss of the power to digest food. Outdoor exercise is particularly helpful as an aid to digestion. Hor'seback riding, bicycle riding, and rowing are to be highly commended.

Mental Impressions. - The digestive process is very greatly under the control of the mind. The connection between the mind and the stomach is so intimate that $V$ an Helmont maintained for a long time that the stomach was the seat of the soul. By any strong emotion the whole digestive apparatus may suddenly cease to act. Fear, rage, and grief check the salivary 
secretion, and without doubt the gastric also. Through the mind, the appetite may be either encouraged or quite destroyed. It seems very plansible that a strong nervous impression might so affect the system as to lay the foundation for chronic dyspepsia.

A man who sits down to dinner with his mind depressed with business cares, the embarrassment of debts, or the anxiety of doubtful speculations, cannot hope to digest the most carefully selected meal. The woman who dines with her mind disturbed with discontent, fretfulness, and worriment, is certain to suffer with indigestion. Domestic infelicity may well be counted as at least an occasional cause of digestive derangements. Meals eaten in moody silence are much more apt to disagree with the stomach than those which are accompanied by cheerful conversation. A hearty laugh is the very best sort of a condiment. Cheerfulness during and after meals cannot be too highly rated as an antidote for indigestion.

Drugs. - The continued use of drugs of various kinds, and especially of patent medicines, "bitters," and purgatives, particularly the latter, has a very damaging effect upon the stomach and bowels. Too much cannot be said to discourage the use of laxatives, purgatives, "liver pills," etc. While sometimes beneficial, agents of this kind, if used for any length of time, are quite certain to work mischief. Purgatives should never be used except as temporary palliatives. If the bowels require artificial aid, the enema is far preferable ; and yet this plan also has its inconveniences, and results badly if too long continued. In general, the less drugs are used, the better. Patent 
nostrums should be shumned like the most virulent poisons, as in many instances they are.

Alkaline laxatives and laxative mineral waters, when used for any considerable length of time, are exceodingly damaging; they irritate the solar plexus and induce hypopepsia.

Sexual Abuses. - Although this is not the place for a dissertation on the subject heading this paragraph, sexual transgression in all its forms must be set down as a not infrequent cause of the class of disorders under consideration. Secret vice in the young, though often unsuspected, is the undermining influence which works irretrievable ruin in many constitntions, weakening the stomach by exhausting the nervous energies upon which it depends for support. Marital excesses have the same effect, and in essentially the same way, the vital forces being exhansted more rapidly than they can be replenished. The researches of Brown-Sequard and his successors have shown that continence is not only consistent with perfect health, but that the secretion of the sexual glands is an important vital stimulus, which is of great benefit to the individual, and indispensable as a controlling element in the development of the body.

Disease of Other Organs.- The nervous connections of the stomach and its associated organs are so extensive and numerous, involving, of course, equally extensive and various sympathies, that it is not a matter of surprise that disease of other parts, through direct sympathy, or through a general influence upon the whole system, often occasions disease of the stomach. This is so generally true that it may almost be said that 
"every disease has its accompanying dyspepsia." This fact is particularly observable in the weakness of the digestive organs which usually follows prolonged fevers ; which accompanies and follows malarial diseases ; and which is closely connected with rheumatic and gouty affections, with most maladies requiring enforced rest for a great length of time, with most forms of brain disorder, and with nearly every form of organic disease, as well as with structural disease of the stomach, as simple dilatation, chronic ulcer, contraction, cancer, and other abnormal growths.

Inherited Dyspepsia.- Many patients assert that they have inherited dyspepsia from a dyspeptic father or mother, having suffered from their earliest recollection from distress after eating, or other disturbance of the digestive functions. In many of these cases there is doubtless an inherited weakness of the stomach, which may become genuine dyspepsia by a very slight deviation from the laws of good digestion; yet it can hardly be supposed that dyspepsia itself is inherited in the sense that some other diseases are. The tendency or predisposition may be inherited in the form of a weak stomach; but in most, if not all, of these cases, careful inquiry will show that the disease itself is due to bad feeding or some other mismanagement in infancy. The use of nursing-bottles without proper attention to cleanliness, which is indeed impossible when a long tube is used; the employment of deceptive mixtures sold as "infant food;" and especially the use of paregoric, "Mrs. Winslow's Soothing Syrup," "worm teas," vermifuges, and other powerful drugs, with an infinite variety of teas, sirups, and other patent and domestic 
compounds, - these are some of the more powerful influenees which occasion early dyspepsia, and often entail lifelong misery and suffering. An individual who has grown up to manhood or womanhood under the gloomy shadow of an ever-present, depressing, despair-produeing dyspepsia, can nerer fully see the sunny side of life, even if his stomach could be made to do its duty. The mind falls into habits of thought and receives a certain cast in early life that no inflnence of after-years can erase. Hence a responsibility rests upon those who have the care of the diet of human beings at the beginning of life which is really fearful, in view of the immediate and remote consequences to the victims of improper nianagement.

A number of observations made by the writer have led him to the conclusion that dilatation of the stomach, a common condition in cases of chronic dyspepsia, often begins in infancy or early childhood. The overfeeding of children and the overdistention of the stomach with gas, - the natural resuit of the use of candy, sweetmeats, cake, and various other unwholesome and indigestible foods, - frequently result in permanent injury to the stomach, the consequence of which is lifelong suffering from indigestion and all the various inconveniences of constitutional maladies arising therefrom.

Undetermined Causes.- No donbt there are many causes of disorders of the stomach which affect it indirectly, throngh the nervous system, and have not as yet been sufficiently studied to receive their due weight. Among these may probably be classed such influences as atmospheric or meteorological changes. I have often met with individuals who, though having 


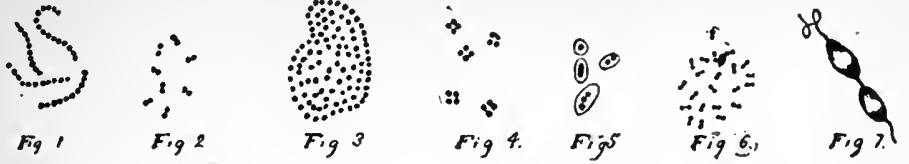

$\sum_{\text {F.g.8., F.g } 9, \text { Figie }}^{10}$

רוֹ

ओ)

है।

5,915
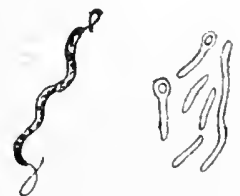

F.g 16

$\therefore g 17$
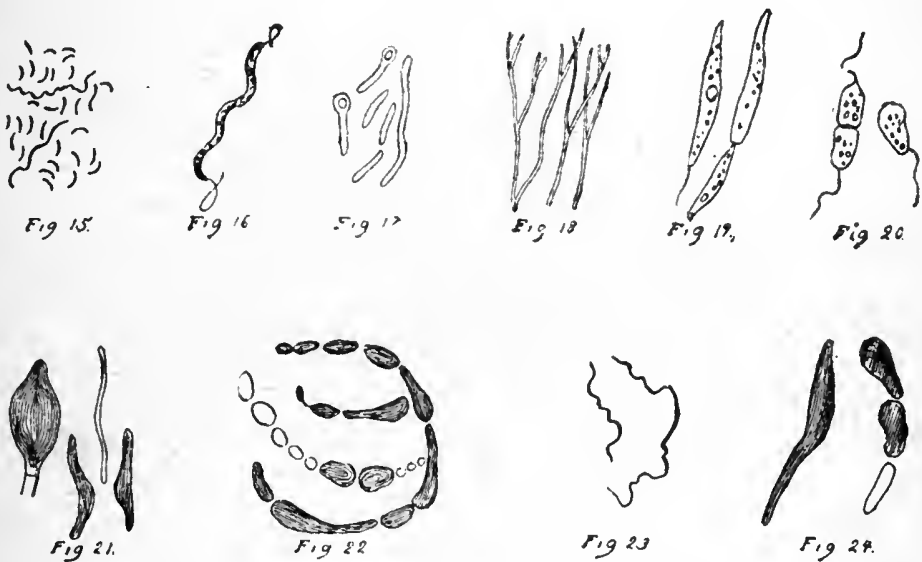

Fig 23

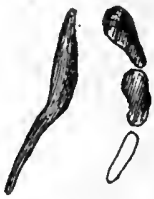

Fig 24.
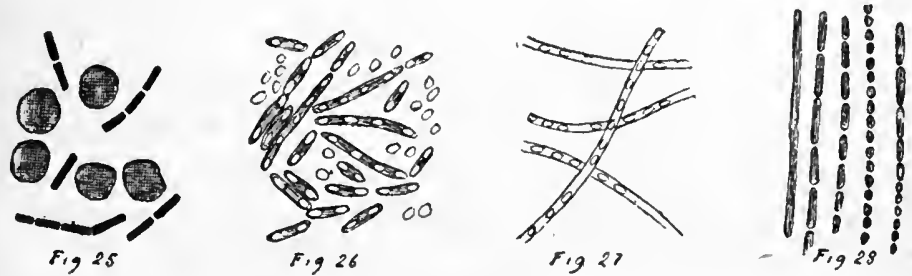


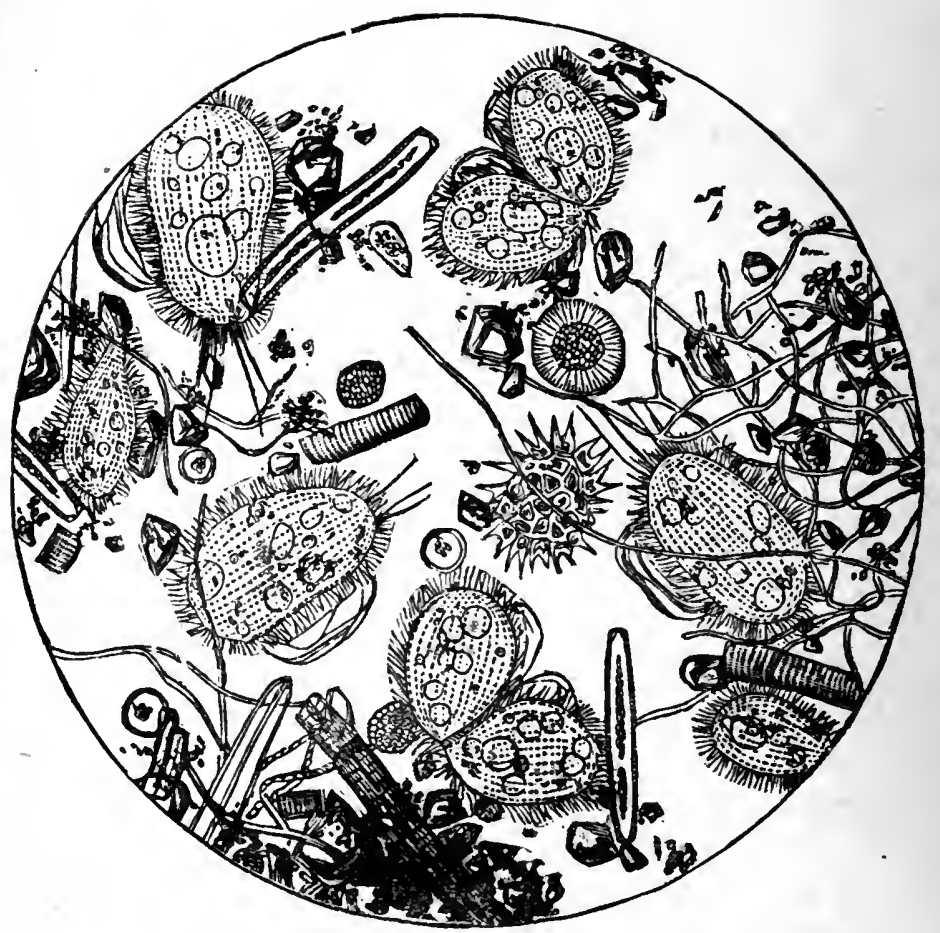

Animalcules and Infusoria in Water.

PLATE III. 
no difficulty with digestion ordinarily, whenever a falling barometer indicated diminished atmospheric pressure, and clouds covered the sky, would suffer great disturbance of digestion. Similar results will, in some cases, almost invariably follow the electrical disturbances which precede and accompany a thunder shower.

Impure Water. - The discovery of the fact that most of the symptoms of indigestion are due to the development of germs in the stomach and the inflnence of the poisons which they produce upon the stomach and remote organs, through their absorption into the blood and their action upon the sympathetic nerves, has made very clear the relation of impure water to digestion. Impure water always contains germs capable of setting up fermentation and putrefaction in food substances within the stomach. Cold water taken into the stomach checks the secretion of the gastric juice and the process of digestion, thus giving the germs introduced with the water an opportunity to set up fermentative and putrefactive changes in the food, whereby are produced acidity, flatulence, biliousness, and sometimes even nausea and vomiting.

The accompanying plate (Plate II) presents some of the more common forms of germs with which the modern science of bacteriology has rendered us familiar. Plate III shows some of the numerons forms of small organisms, animal and vegetable, which are to be found in water obtained from natural sources. 


\section{IMPORTANT NEW DISCOVERIES RE- LATING TO DIGESTION.}

Within the last twenty-five years, but especially during the last decade, immense progress has been made in the study of the disorders of digestion, and especially in the methods of examining both the stomach as regards its physical condition, and also the products of digestion. This subject is somewhat too abstruse for the unprofessional reader ; but a brief resumé of some of the more important facts relating to recent progress in methods of examination of the stomach and investigation of its disorders can scarcely fail to be interesting even to nonmedical readers.

The most important discovery which has been made in modern times in relation to the examination of the stomach is the method sometimes termed "tubage," which consists in introducing into the stomach a soft or semi-soft and very smooth and flexible rubber tube. This tube is not forced into the stomach, as was the case with the tube of the old-fashioned stomach-pump, but is simply placed in the mouth so that the end of the tube touches the. back part of the throat, where, being grasped by the muscles of the throat in the act of swallowing, it is quickly carried down into the stomach. By means of the stomach-tube it is possible, by an actual measurement of the quantity of fluid which the stomach will hold, to form a very accurate estimate of its size. It is also possible to determine whether the stomach empties 
itself completely, and how long a time elapses after eating before the stomach has disposed of its food contents by passing the digesting food along into the small intestines. Many other facts relating to the work of the stomach can be determined by this means, but these will be spoken of later.

Various methods have also been devised for deter. mining the size and location of the stomach without the use of the stomach-tube. One of the most ingenious of these is the distention of the stomach with gas by generating carbonic acid gas in the stomach. For this purpose the patient is first given bicarbonate of soda dissolved in a little water, then tartaric acid, also dissolved in water. The meeting of these two substances in the stomach gives rise to the development of a quantity of carbonic acid gas. The quantity of chemicals introduced must be varied somewhat, of course, according to the age and the size of the patient. This method is not very much used, however, on account of its inconvenience, and the possibility of injury, especially in cases of ulceration of the stomach, in which serious damage might be done by a rupture of the weakened stomach walls at the seat of the ulcer.

Another method of determining the position of the stomach is that known as clapotement, which was devised by a French physician. This method depends upon the fact that agitation of the stomach when both fluid and air are present in it, gives rise to plashing sounds which can be easily heard by the unaided ear, and can be very accurately located by means of the stethoscope, an instrument used also for examination of the lungs and the heart. 
Another method, which the author had the good fortune to discover a few years ago, is what might be termed "simple palpation." By this method careful examination of the stomach is made with reference to the presence or absence of fluid. If plashing sounds are not heard, then the patient is made to drink water as before. After the water has been taken, the physician, standing upon the right side of the patient, places his right hand upon the abdomen just below the ribs of the right side and to the right of the median line ; while the fingers of the other hand are placed upon the corresponding point of the left side. Movements are then made by the right hand of a character calculated to agitate the contents of the stomach; while with the left hind careful observation is made of the,presence or absence of the sign of "fluctuation." In case the patient is not too fleshy, the movement of the liquid from one side of the stomach to the other, resulting from the agitation with the right hand, gives rise to inpulses which are readily recognized by the left hand, as the fluid set in movement by the right hand impinges against the wall of the stomach, and communicates the impulse to the abdominal wall of the opposite side. After some practice the movements of fluid in the stomach can be readily detected, and, by directing the impulses in various directions, the whole outline of the lower border of the stomach may be clearly defined.

This method seldom fails except in very fleshy subjects. In order to locate the stomach in these cases, the author has constructed a special instrument, which consists of a stomach-tube, bearing at its inner extremity a small electrical contrivance which is capable 


$$
\therefore
$$



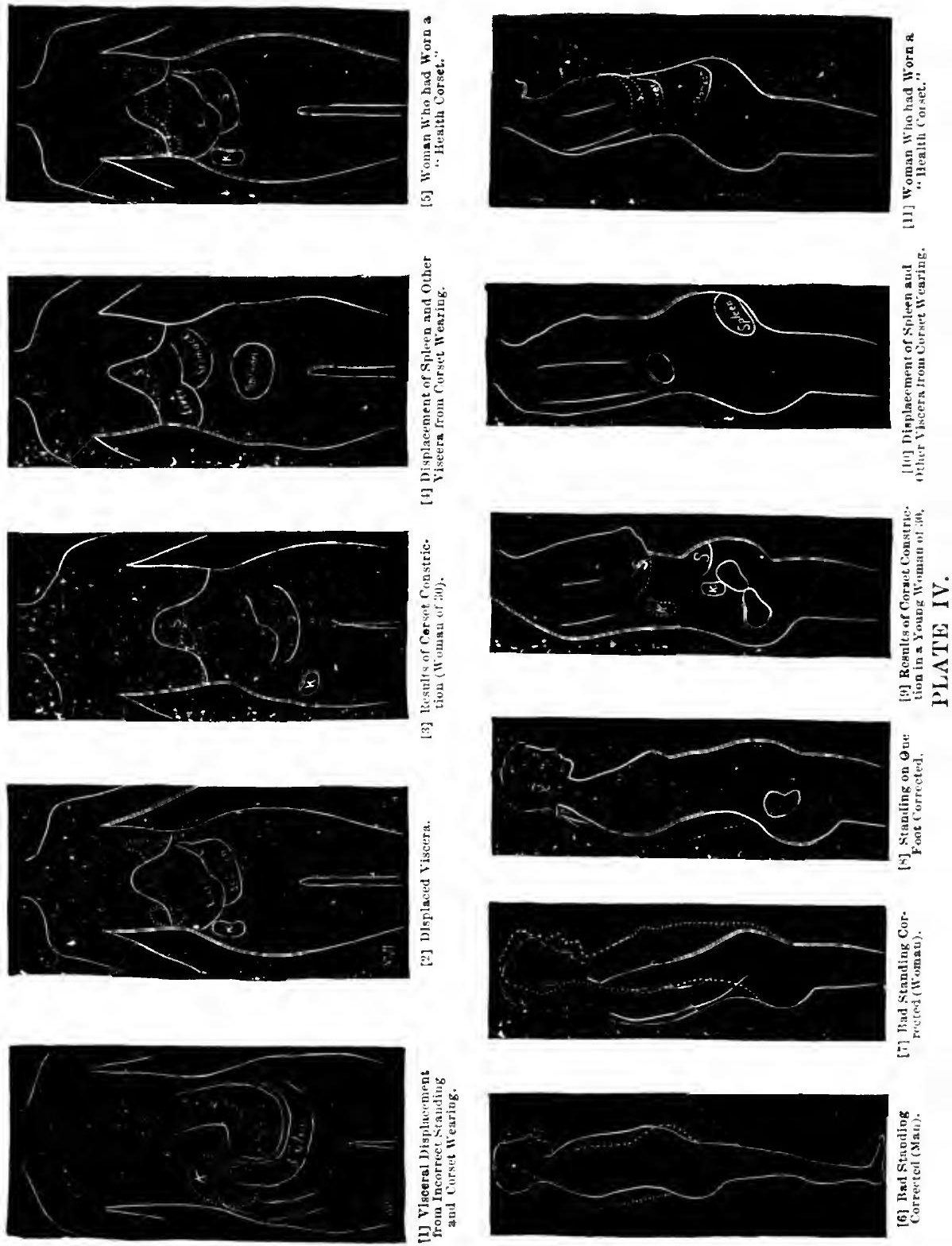

宫.

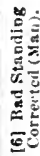


of emitting a clicking or buzzing sound when excited by an attenuating electrical current. Passing the tube inside, and listening with the stethoscope while the indicator is in position, it is very easy to determine the lowest limit to which the tube can be passed; and by making movements or readjustments of the instrument, the outline of the lower border of the stomach can be very accurately marked out.

This is the method commonly employed by the writer in making his examinations of the stomach. By this method it is possible for one sufficiently experienced to readily mark out the location of the lower bqrder of the stomach in a few seconds, and to discover a prolapse of the stomach, dilatation of the stomach, or a pocket in the stomach,- conditions one or more of which are to be found in-a great majority of the cases of chronic dyspepsia.

The influence of these abnormalities in size and position upon digestion will be referred to later. The accompanying cuts (Plate IV) are illustrations of a few of the abnormalities which the writer has noted in his examining office. In one of these cases, as will be noticed, the stomach was prolapsed nearly to the pubes. The colon, kidneys, and all the other viscera of the abdomen were likewise in a prolapsed condition. The kidney, in this particular case, was prolapsed to such a degree that its function had been interfered with, and it had become so badly diseased that it was found necessary to remove it. Within the kidney was found a stone weighing four and one-third ounces. The disease was evidently the outgrowth of the malposition of the kidney. Prolapse of the stomach and dilatation of the 
stomach and colon are conditions to whieh too little attention has been given. It is probable, also, that prolapse of the bowels and dilatation of the stomach are sometimes the result of indigestion; but prolapse of the stomach must certainly be, in many cases, the cause of disturbance of the digestive function, being itself the result of such mechanical causes as waist constriction from corset-wearing, tight waistbands, or the wearing of belts.

\section{Examination of the Stomach Contents.-} The examination of the contents of the stomach obtained after a test meal is of still greater importance than a physical examination, - - at least in the treatment of functional or non-structural diseases of the stomach. The stomach contents are obtained by means of a stomach-tube, which has already been described. The patient is given a test meal consisting of one and one-half ounces of dry water-bread or two ounces of ordinary baker's bread, with eight ounces of water. Exactly one hour from the beginning of the meal, the tube is passed into the stomach and the contents withdrawn. This is accomplished much more easily than might be imagined; indeed, few persons suffer much from the withdrawal of the test meal, although in occasional instances the attempt to introduce the tube provokes a spasm in the throat which renders the passage of the tube impossible.

After obtaining the stomach fluid, it is subjected to a most critical and elaborate examination. The method of examination, which will be more fully described, has been compiled, arranged, and perfected by the author 
from the investigations in this direction by many other scientific workers. Some portions of the method are quite new, being the result of the author's personal work and experience. This is especially true in reference to the methods of determining coefficients, which, with the exception of one, have all been devised and determined by him.

The methods employed in testing the digestion of starch, while not new to chemistry, have not been previously employed in studying the disorders of digestion. The facts determined in this method of investigation, which is more elaborate and thoroughgoing than any other with which the writer is acquainted, will be found in a colored chart near the close of this book, together with the figures obtained in an actual case examined, in which the amount of work done by the stomach was greater than normal. This method of investigation has been developed as the result of years of patient research and inquiry on the part of many different investigators in different parts of the world. The foundation for the method employed was laid by Dr. Golding Bird, of Guy's Hospital, London, more than fifty years ago, in the discovery that the acid of the gastric juice is hydrochloric acid, and that the degree of activity of the stomach in the digestion of albumen, is ehiefly dependent upon the presence of this acid. The hydrochloric acid of the gastric juice has been shown, however, to be not simply the ordinary chemical compound known as hydrochloric acid, or muriatic acid, but an organic hydrochloric acid which is possessed of much greater activity as a digestive agent in combination with pepsin than is ordinary hydrochloric acid. 
Dr. Bird's researches were within a few years reviewed by Hayem and Winter, two French physiologists, who perfected methods for determining not only the amount of hydrochloric acid present in a given specimen of gastric juice, but the amount and quality of the work done by the stomach in the digestion of albumen. Ewald, Boas, and other German physicians have contributed important methods for determining other facts. Professor Roberts, of England, has established a convenient method of studying starch digestion. The writer, though not pretending to any special eminence as a chemist or an originator of new methods of study, has undertaken to combine these various methods, and by the assistance of skilled chemists and the advantage of experience in the examination of more than five thousand cases has perfected the method of studying disorders of digestion herewith presented. A brief explanation of the several facts determined by this method will be of interest:--

1. The amount of stomach fluid is normally forty cubic centimeters, or one and one-third ounces; that is, of the eight ounces of fluid and the one and one-half to two ounces of solid food, taken into the stomach, all, with the exception of one and one-third ounces, should be absorbed at the end of one hour, or pass on into the small intestine. If a larger amount is found, the indication is slow absorption; if a smaller one is found, the absorption is more rapid than normal.

2. In the examination of a large number of cases the average residue left after filtration, is found to be, in healthy persons, twenty grams, or about one half the total amount obtained. A larger residue than this, 
or a larger proportion of residue, indicates deficient activity of the digestive fluids in dissolving the food, or delayed solution.

3 . If the residue presents masses of food of considerable size, it is evident that mastication has been insufficient.

4. The presence of a large amount of mucus indicates gaštric catarrh.

5. A greenish or yellow color usually indicates bile ; a red color, blood.

6 . The odor of putrescence indicates decay ; a sour odor indicates the presence of acetic acid.

7. A red color, or the presence of any unusual appearance, should lead to a careful microscopic examination by which the presence of pus or germs may be readily determined. A thorough examination of the germs or microbes present includes a cultivation of the germs by mixing a few drops of stomach fluid with a proper culture medium, and subsequent staining or microscopic examination by ordinary bacteriological methods.

8. The digestive agents which have been found present in the stomach fluid are next investigated by means of color and other reactions. Those of the most importance are hydrochloric acid, pepsin, and the rennet or lab-ferment. Examination is also made for the presence of bile. If any of these substances are absent, the effect is indicated by zero; if present, by a simple + sign ; if in an unusual quantity, by ++ .

9. Recent discoveries in physiological chemistry have made it possible to investigate with almost perfect accuracy the different products of the digestion of 
albumen, or rather the products which are found in the different stages of the conversion of albumen into peptone, the final product of the digestion of albumen.

10. By means of the reaction with a solution of a compound of iodine, a very good idea may be obtained respecting the conversion of starch into dextrin or maltsugar, the reaction showing in succession the colors, blue, violet, brown, and yellow while the change is taking place, until the solution becomes colorless.

11. By methods which have been largely developed in the Sanitarium Laboratory of Hygiene, it has been found possible to obtain very definite data respecting the amount of fermentation which may have occurred in the case of any particular stomach fluid. Of the prodnets of fermentation found in the stomach, the most common are lactic and acetic acids. Other acids and alcohol, are, howerer, sometimes found.

12. The above determinations are all of interest and importance, but still more so are the more exact determinations made by quantitative analyses of the stomach fluids, whereby the degree of acidity is accurately determined, together with the amount of chlorin found in the different conditions, free, combined, and fixed. The significance of this will be understood by the simple mention of the fact that the gastric juice depends, for its digestive activity, upon free hydrochloric acid, and that when fermentation is not present, the more strongly acid the gastric juice, the greater its digestive vigor, other things being equal.

-13. The determinations with reference to starch have been undertaken on an extensive scale. At the 
present writing, these determinations have been made in nearly one thousand cases in the Sanitarium Laboratory of Hygiene, under the author's direction. The determinations made, are (1) with reference to the saliva, whereby the quantity formed and its activity are determined; (2) the amount of malt-sugar or maltose, or completely digested starch, formed in the stomach ; (3) the amount of dextrin and soluble starch, or the imperfectly digested starch.

14. By accurate chemical methods the total quantity of acils resulting from fermentation, including lactic and acetic acids, is determined.

15. A careful bacteriological study is made of the stomach fluid for the purpose of determining the number and kind of germs present, the number found varying from none in a healthy stomach to many millions in an infected stomach. This I have found to be altogether the most exact method of determining the condition of the stomach as regards infection and the resulting tendency to fermentation.

The experiments made show conclusively that fermentation is not a natural process in connection with digestion, and emphasizes the importance of suppressing germs in the food, so far as possible.

The following facts are readily determined by this examination :-

(1) The relative number of germs present.

(2) The character of the germs, and the nature of the disturbance which they produce in the stomach.

16. Most interesting of ail are the determinations relating to the coefficients of digestive work. These coefficients are worked ont from the data furnished by 
quantitative analysis. The indications of these coefficients are as follows:-

a.- This coefficient indicates the quality of the work done by the stomach in the digestion of albumen. The normal is 1.00 , as in the case of each of the coefficients. In the present case the coefficient is found to be .83 , indicating that the work done by the stomach in the digestion of albumen was much below par. Still another fact, however, is dereloped by this determination; the difference between the coefficient and 1.00 indicates, in each case the proportion of the vicious products of the digestion of albumen formed in the stomach and thrown into the circulation. With a coefficient of .50 the indication would be that one half the albumen digested in the stomach was so imperfectly digested as to be of no value as food, but constituted, instead, a mass of poisonous substances which, after absorption into the blood, must be destroyed by the liver and carried out of the body through the kidneys.

b. - This is the coefficient of starch digestion, and indicates how nearly the process of starch digestion in the stomach approaches completion.

c.-This coefficient indicates the degree of efficient activity of the salivary glands. It expresses both the quantity and the quality of the saliva.

x.-This is the coefficient of fermentation. It indicates the relation of the acidity due to abnormal acids to the total acidity.

y.--This is the coefficient of solution, and indicates the degree to which a given case approaches the normal standard of twenty grams of residue to 40 c.c., or one and one-third ounces, of stomach fluid. 
z.-This is the coefficient of absorption. It indicates the quantity of fluid found, or its approach to the normal amount. An excess of fluid indicates slow absorption.

m.- This coefficient indicates the degree of activity of the stomach in producing hydrochloric acid, one of the most important constituents of the gastric juice.

The above data in relation to the stomach fluid obtained after a test meal have been deterinined in the . Laboratory of the Battle Creek Sanitarium, under the author's supervision, in more than five thousand cases, each one requiring more than fifty different accurately executed chemical processes.

It is impossible here to enter into an elaborate discussion of the bearing of all these important data upon the functional disorders of digestion and the treatment of those disorders. The reader will, however, be able to see at a glance that accurate determinations of this kind must render possible a more exact adaptation of remedies and diet to individual cases than is possible without such definite and precise information.

Twenty years ago, when the writer was starting out in the medical profession, one of the greatest difficulties and embarrassments which he encountered, was the lack of definite information respecting the disorders of digestion and the adaptation of remedies to their relief. Prescriptions, diet, treatment, and regimen were almost altogether matters of guess-work or intuition; and, in many cases, the only way of finding out whether the patient required one dietary or another, was to experiment upon him. A recognition of this fact caused an eminent New York physician to declare that every dose of medicine was a blind experiment upon the vitality of 
the patient.' A new case of chronic dyspepsia was always encountered with dread, on account of the tedious experimental process, painful alike to both physician and patient, which must be gone through in the discovery of just the dietary and the treatment best adapted to the case. By means of the present method, howerer, it is possible to obtain such accurate information that treatment and a dietetic prescription may be made with full confidence that the patient will be afforded definite and prompt relief.

One of the most important advantages gained by this precise method of analysis is the possibility of making an exact and definite classification of all the functional disorders of digestion. In relation to this new classification, it may be said briefly that, considered in relation to the amount of work done, there are four general classes of indigestion,--hyperpepsia, in which there is excessive stomach work; hypopepsia, in which there is deficiency of stomach work; apepsia, in which there is no digestive activity whatever; and simple dyspepsia, in which the amount of work done by the stomach is normal, the change in the stomach work being qualitative rather than quantitative. By these studies I have been enabled to subdivide these several classes as follows:-

Iyperpepsia, into three principal classes and twelve subclasses.

IIypopepsia, into two principal classes and eight subclasses.

Apepsia, into two subclasses.

Simple dyspepsia, into four subclasses. 
This makes twenty-six different varieties of indigestion, each with its individual characteristics.

In the results of the study of over five thousand analyses made with painstaking accuracy by the methods which I have very briefly described, I have not found a single case which did not fall in one of the classes named, and have found examples of every class.

I give, in the following pages, an abbreviated outline of the classification, together with a brief outline of the data determined by the thorough investigation of the work done by the stomach which the modern advances in chemistry and bacteriology render it possible to make.

It must not, of course, be supposed that the treatment of disorders of digestion can be made, even by these exact methods, in any way mechanical or routine, since every individual case presents peculiarities which must be taken into consideration. The withdrawal of the contents of the stomach in the midst of the process of digestion, enables us to surprise the stomach at its work, and thus to detect special faults, such as excess or deficiency of activity, abnormal fermentations through the development of germs in the stomach, etc., and thus makes clear much which has heretofore been a mystery, or a matter of pure speculation in relation to the disorders of digestion. Nevertheless, the size, shape, and location of the stomach in relation to normal conditions, and many other details, must be taken into consideration in formulating a prescription. 


\section{CLASSIFICATION OF FUNCTIONAL DISORDERS OF THE STOMACH}

\section{IN RELATION TO PROTEID DIGESTION,}

\section{Based upon a Quantitative Chemical Examination of the Stomach Fluid Obtained after a Test Meal, in over 5,000 Cases.}

The symbols under each head indicate the characteristics of each individual form of quantitative disturbance to which the stomach is subject.

\section{HYPERPEPSI. $\mathbf{A}^{\prime}+$}

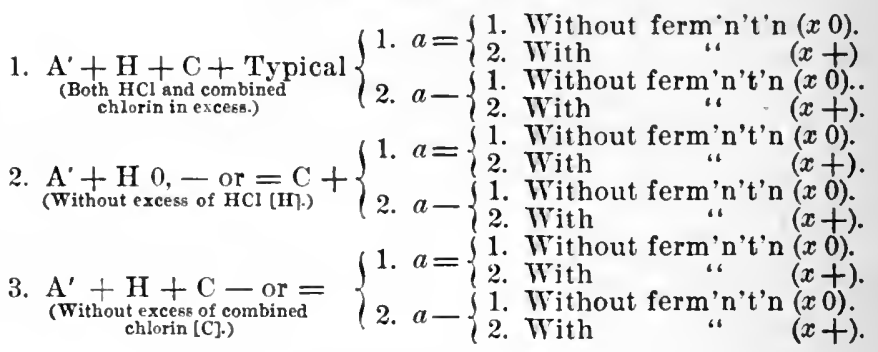

\section{HYPOPEPSIA. $\mathbf{A}^{\prime}$-.}

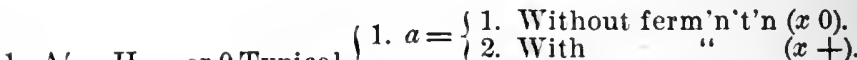

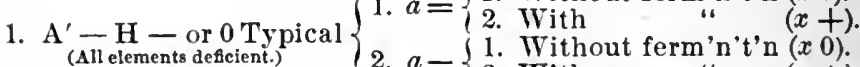

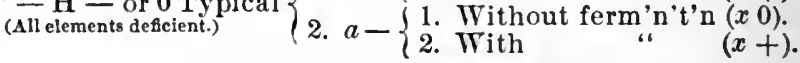

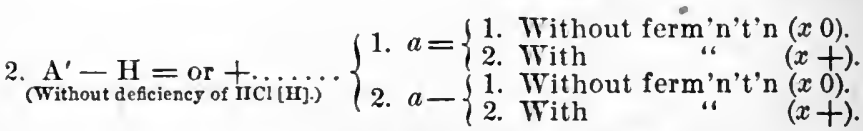

APEPSIA. A o or $-\mathrm{a}$ o or $-\mathrm{H}$ o.

1. Without ferm 'n't'n $(x 0)$.

2. With " $(x+)$.

SLMPLE DYSPEPSIA. $A^{\prime}=$.

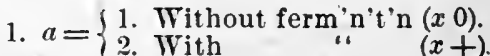

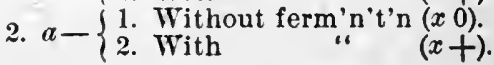


RESULTS OF QUALITATIVE AND QUANTITATIVE DETERMINATIONS RELATING TO SALIVARY DIGESTION AND THE STOMACH FLUID OBTAINED AFTER A TEST MEAL.

(The results given below represent the actual facts founc in a case of hyperpepsia as taken from the author's office file.)

Case No...5284. .

M iss B

Date....Aug. 25,...189.5. .

Test Meal. - Regular Test Breakfast................... Time of Digestion....1...h....13...m.

\section{PHYSICAL AND MUCROSCOPICAL CHARACTERS.}

Physical. Normal.

Amount..95..c.c. (40 c.c.); Disintegration..t..: Color..n..

Residue..25..grms. (20 grms.); Mucus .... ....; Odor..n.. Microscopical._Blood......; Pus.....; Misc.....; Bacteria: No......per c. c.; Character......

\section{COLORIMETRIC AND OTHER QUALITATIVE REACTIONS.}

DIGESTIVE AGENTS.

Free HCl Congo Red. . . . Resorcine... . . Dried Residue.. . . Pepsin.... Rennet Ferment. .. Rennet Zymogen. ... Bile.0.. DIGESTIVE PRODUCTS.

Proteids._Syntonin....+.. Propeptone...+.. Peptone...+.. Albuminoids.....

Starch (Lugol's Sol.), Blue, Violet, Brown, Yellow, Colorless.

FERMENTATION PRODUCTS.

Lactic Acid........

\section{QUANTITATIVE CHEMICAL DETERMINATIONS.}

DIGESTION OF ALBUMEN.

Total acidity, (A ) ..... .314...grms. (.180-.200 grms.) $A \dagger$

Calculated acidity, $\left(A^{\prime}\right)^{* *} .362 \ldots$. " " " " $A^{\prime}$

Total chlorin, (T) ......390 .. “ “ $\quad(.300-.330$ “ )

Free HCl, (H)........ 042... " " $(.025-.050$ " ") $H$

Combined chlorin, (C)... .320.. " " $(.155-.180$ “ ) $C$

Fixed chlorids, (F)...... .032.. “ “ $\left(.100-.110 \quad\right.$ “) $\frac{T-F}{T}=.66$ 
DIGESTIOY OF STARCH.

Saliva, a m't in $5 \mathrm{~m} . .11$..grms. (1 c.c.) converted .1 grm. in. .13. .m. Maltose, (II) ....................... 1.424...grms.

Dextrin and Soluble Starch (D) $\ldots \ldots \ldots \ldots \ldots . . .616 \ldots$...

Fatty Acids (from fermentation) (L)......... .005... “

(Lactic acid...006..grms. Volatile acids .....grms)

\section{COEFFICIENTS OF DIGESTIVE WORK. $\nmid \dagger$}

Gastric, Proteids $(a) \ldots \ldots .83 \ldots$ Fermentation $(x) .6 \ldots$ Digestion $\{$ Starch $(b) \ldots \ldots \ldots .24 \ldots$ Solution $(y) \ldots \ldots$ 1.48 .. Salivary Activity $(c) \ldots \ldots \ldots .42 \ldots$ Absorption $(z) \ldots \quad .42 \ldots$ Coefficient of Chlorin Liberation $(m) \ldots \ldots \ldots \ldots \ldots \ldots$. 1.38 .. Proteid Digestion and Acid Fermentation $(a$ and $x) \ldots \ldots, 1.03 \ldots$

Diagnosis. - ......Hyperpepsia with fermentation (2-2-2) and imperfect digestion of starch.

* The acidity ( $A$ and $A$ ) and the quantities of chlorin in the different forms of $(\mathrm{T}),(\mathrm{H}),(\mathrm{C})$, and $(\mathrm{F})$, are expressed as $\mathrm{HCl}$. The values given relate to 100 c.c. of stomach fluid. The amount of fatty acids, chiefly lactic (L), is expressed in grams of $\mathrm{HCl}$, which should be multiplied by 2 to indicate the actual amount of lactic acid present. The a mount of maltose (M), as well as that of soluble starch and dextrin(D), is expressed in grams of equivalent dextrose.

+ By noting the sign following the symbols in this column and coeffeients $(a)$ and $(x)$, and referring to the classification, the diagnosis of any case in relation to proteid digestion and fermentation may be easily made. By transferring the figures to the blank for "Graphic Representation," the indications will be secn at once, the analysis thus furnishing a basis for rational treatment and the proper dietary.

** The calculated acidity (A) is determined by the following formula: $\mathbf{C}+\mathrm{H}-\mathbf{L}=\mathbf{A}^{\prime}$.

++ The normal amount of work is, in the case of each coefficient, represented by 1.00. The figures given usually represent the percentage of devlation from normal. The coefficient of fermentation represents the number of milligrams of lactic acid or combined fatty acids found in each 100 c.c. of stomach fluid (expressed in equivalent $\mathrm{HCl}$ ).

The sign + indicates presence in normal quantity $;++$ in excessive quantity; - in deflcient quantity ; 0 (zero) wholly absent.

As regards the relative frequency of the different classes, simple dyspepsia is the most common of all. Apepsia is quite rare. I have met less than fifty cases 
in more than five thousand, or a little less than one per cent. Hypopepsia and hyperpepsia are about equally frequent.

As regards recognition of these various classes by symptoms alone, I am sorry to be obliged to say that it is impossible. A test breakfast and a careful ex- amination such as I have described is absolutely essential to a precise diagnosis of the functional disorders of digestion. One who has studied a large number of cases by the method described, may, by a careful study of the symptoms, sometimes form an approximately correct judgment in respect to the nature of the case in hand without making a chemical examination; but positive information can be obtained by no other method than by exact chemical analysis. A few points relating to symptoms are worthy of mention, as follows :-

In lyperpepsia there is an excessive secretion of gastric juice, in connection with which there is usually a good appetite, but an excessive irritability of the stomach, as is shown by the tenderness elicited by pressure at the pit of the stomach or just at the lower end of the sternum. Other symptoms due to excessive irritation of the gastric mucous membrane are also more common than in simple dyspepsia, hypopepsia, or apepsia. Acidity not due to fermentation is commonly present in extreme cases of hyperpepsia. This form of acidity develops soon after the taking of a meal, gradually increasing until the food is expelled from the stomach into the intestines three or four hours after eating, or until the acid contents of the stomach are washed out with a stomach-tube, diluted 
by the drinking of water or some other fluid, or somewhat modified by the taking of more food, or neutralized by soda, carbonate of magnesia, or some other alkali. Patients suffering from hyperpepsia are often fleshy and florid, although not infrequently they are quite thin in flesh.

In hypopepsia there is often a loss of appetite, general feebleness, pallor, and more or less pronounced symptoms of indigestion, with anemia, or an impoverished condition of the blood.

In apepsia the symptoms of hypopepsia are very much more pronounced.

In simple dyspepsia the principal symptoms may be simply weight at the stomach, and evidences of fermentation.

In case of fermentation, whatever other symptoms may be present, the bacteriological examination shows germs of some sort present in great numbers. In one case investigated in the Laboratory of Hygiene connected with the Battle Creek Sanitarium (Mich., U. S. A.), the stomach fluid was found to contain for each ounce, more than $16,437,300$ yeast cells. Other germs were entirely absent. By the aid of this investigation it has often been possible to make an immediate and successful application of appropriate remedies, which, without this method of learning the exact conditions present, could only have been reached by a long and perhaps disappointing experimental effort. 


\section{SYMPTOMS OF DYSPEPSIA.}

As nearly every disease may include among its symptoms some disorder of digestion, so dyspepsia may include in its symptoms some of those of nearly every disease that could be named. This is readily understood when we consider the fact that impairment of digestion interferes with the nutrition of every organ of the body. Every part suffers, and of course the suffering organs express themselves in the various symptoms by which they manifest diseased or disordered functions. The particular features manifested in any given case will differ with individual peculiarities of constitution or temperament, which give prominence to some particular set of symptoms which may or may not refer to the stomach. As a usual thing, however, there are certain symptoms which refer directly to the stomach, and which are generally understood as indicating disordered digestion. The intensity of the symptoms manifested varies from the slight uneasiness and sense of weight or fulness occasioned by a small excess of eating, to the most distressing and painful condition of the more aggravated forms of the disorder.

When possible to do so, the treatment of every case of disordered digestion should be preceded by a careful examination of the stomach fluid, which gives information obtainable in no sther way, and which can 
be relied upon as exact. It is not always necessary that the patient should visit a laboratory in order that an examination be made. Most modern physicians are provided with suitable stomach-tubes, and can administer the test breakfast and remove the stomach fluid at the proper time, place it in a clean bottle, carefully seal and pack the same, and send it by express to a laboratory in which such examinations are made. Stomach fluids usually keep without material change for some little time. (For directions for test meal, see page 134.)

As this work is especially intended, howerer, for persons who are so situated that they cannot avail themselves of skilled medical advice, and since the home treatment of this disease must be largely symptomatic, we present a simple classification based upon symptoms, and a detailed account of the principal symptoms of dyspepsia, together with the best methods obtainable at home for the cure of the disease.

Classification of Indigestions.- Dyspepsia may be classified, first, as acute and chronic. One of the most important differences between an acute and a chronic case of indigestion is that in acute dyspepsia recovery will occur in time, usually in a very short period, by the unaided efforts of nature; while a chronic case of the disease continues from bad to, worse, or without material improvement, indefinitely.

Most cases of acute dyspepsia are the result of excess in eating, taking food at an unseasonable hour, the partaking of unwholesome and indigestible substances, or the accidental swallowing of some highly irritating substance, as poisoned or decayed food, or 
some similar irritant. The majority of these cases recover spontaneously, and so quickly that they scarcely need further attention in this connection.

Chronic dyspepsia is generally much less active in its symptoms than is the acute form of the disease. It usually begins slowly, making its advances insidiously, and thus for a long time eluding observation, in many instances until well established. This is one reason why the diagnosis of the disease is often obscure. Very frequently it is overlooked for years, being mistaken for some other discase through the special prominence of symptoms elsewhere than in the stomach.

Basing the classification of chronic dyspepsia upon the most prominent symptoms observed in different cases of the disease, by far the greater proportion may be inclnded in the following five classes; viz., simple, acid, septic or bilions, painful, and nervons dyspepsia.

Each of the classes named has its characteristic symptoms, though any given case may combine the symptoms of one or of all the different classes. After a brief consideration of the most important symptoms relating to the stomach which appear in different phases of the disease, we will consider the special symptoms, or groups of symptoms, which characterize the different classes of dyspepsia mentioned.

Principal Symptoms of Indigestion.-The different symptoms observed in the disorders of digestion arise from -

1. Disturbance or irritation of the nerves of the stomach.

2. Disturbance of the muscular apparatus, or motor functions, of the stomach. 
3. Disturbance of the secreting, or glandular, functions of the stomach.

4. Change in the size and location of the stomach, as in dilatation and prolapse.

In addition to these, there are also physical symptoms relating to the tongue; symptoms pertaining to the evacuation of the stomach and bowels, vomited matters, and stools; symptoms which pertain to the rectum; and various general and remote symptoms which are the outgrowth of the disorders of digestion. A brief enumeration of the most important of these symptoms may be found of practical interest. They are presented here in the order in which the writer commonly notes them in the examination of a patient.

Symptoms Pertaining to the Mouth.-1. Morbid sensations - stinging, burning, loss of taste and morbid tastes, such as metallic, acid, sweet, or peppery sensations ; 2. Secretion - viscid, scanty, or in excess ; 3. Mucous membrane - small ulcers, red points; 4 . Breath — sour, fetid, fecal ; 5 . Coated tongue.

Symptoms Pertaining to the Throat.-Excessive thirst, dryness, irritation, cough, choking.

Symptoms Pertaining to the Esophagus. - Symptoms of burning, constriction, or a feeling of a ball or lump in the throat.

Symptoms Pertaining to the Stomach.-Symptoms arising from disturbance of sensation - distention, constriction, heaviness, burning, coldness, sensation of fulness, beating; abnormal hunger,_constant, sudden, and uncontrollable, frequently requiring food between meals; appetite excessive, diminished, variable, depraved, absent, but returning after taking a little food, 
increasing with meal, insatiable, craving for special foods, disgust for certain foods ; nausea ; pain.

Disturbances of the Motor Functions. - Regurgitation of food, rumination or merycism, eructations of gas, vomiting of fluid, food, blood, pus, bile, or mucus (either with or without nausea); burning, dull, or sharp pain ; faintness ; pain on eating.

The Discharges. - Vomit, — blood, pus, bile, curd, bitter, sour, odor of decay; stools, - hard, soft, or balls, ragged, acid, very foul, black, clay-colored, watery, oily, mixed with mucus, bloody; parasitic worms ; constipation, diarrhea.

Symptoms Pertaining to the Rectum.-Painful stools, burning pain after stool, loss of desire to evacuate the bowels, anal itching.

General Nervous Symptoms. - Nervonsness, morbid fears, depression, loss of memory, lack of energy, inability to concentrate the mind, mental confusion, dulness ; disturbance of spech, as stammering; fidgets, irritability, insomnia, loss of self-control; drowsiness constantly, or after meals; headache at the back, front, top, or side of the head, affecting one or both eyes, constant, periodical, or occisional ; sensation of heaviness, constriction, or discomfort in the head, legs, arms, or other parts of the body ; sensation of abnormal size in arms, head, or legs; strange sensations in the eyes, as strained feeling, a feeling as if bursting from the sockets or sunken in the sockets; desire to support the back of the head; pain in the scalp or in different portions of the spine after drinking or meals; pain between the shoulders; pain in the lower part of the back; pain between the ribs or under the ribs; giddi- 
ness, vertigo ; partial or complete loss of conscionsness due to sudden fright or some strong impression; sensation of lightness in the head; disturbing dreams ; sudden awakening at night with constriction of chest, with difficult breathing or heary beating of the heart; " explosions" in the head; coldness between the shoulders; colduess in hands and feet or knees; sensation of shivering or burning after eating; ringing in the ears; disturbances of vision, unnatural colors ; disturbance of smell or taste; abnormal sensations, such as numbness, tingling, burning, ete. ; sudden loss of strength ; trembling and twitching of muscles; constant weariness; palpitation of the heart; irregular sensations of the heart; sensation of constriction or heaviness about the heart; shortness of breath; nerrous asthma; stomach cough ; hiccough ; in roluntary sighing ; excessive quantities of urine of light color ; scanty urine ; too frequent urination; whitish or reddish sediment in the urine.

Physical Signs. - Physical examination shows in a large proportion of eases of chronic dyspepsia, dilatation or prolapse of the stomach (see page 317 and Plate IV). This condition is often indicated by an unnatural plashing sound heard by the patient in walking or other movements, especially after drinking water, and by unnatural prominence of the lower abdomen.

All the above symptoms and many others of less importance are observed as the result of disordered digestion, although of course all the symptoms named are not present in any single case. 


\section{THE SYMPTOMATIC TREATMENT OF INDIGESTION.}

Uneasiness at the Stomach.-One of the most common symptoms, and one which is frequently the first noticed by patients, is an uneasy sensation in the stomach, which is variously described as fulness, weight, tightness, oppression, heaviness, heat, cold, pulling, "a load on the stomach," drawing, "working," etc. There may or may not be tenderness on pressure at this stage. The uneasiness complained of is greatly increased by any excess in food, and may be occasioned by eren an ordinary amount, by eating too rapidly, by drinking at or soon after meals, or by eating articles of food which are difficult of digestion, as warm bread or biscuit and butter, pastry, fried food, pickles, etc. The essential difference between this condition and that resulting from eating to excess in health, is that the symptoms described are produced by an ordinary and proper amount of food, being only increased in severity by excess in quantity.

These symptoms are all due to an increase of the irritability of the gastric nerves. They may be caused by the use of alcoholic liquors, tea and coffee, ice-cream, iced drinks, too frequent eating, and the use of condiments, as mustard, pepper, pepper-sauce, horseradish, Worcestershire sauce, and in some cases by the use of raw onions, radishes, and fats. 
Treatinent.- These symptoms are generally relieved by an aseptic dietary (Diet List No. 1). In extreme cases the dietary should be confined to well-disintegrated foods (Diet List No. 7). In very obstinate cases, a liquid diet (Diet List No. 11) or a milk diet is indicated. All foods difficult of digestion (Diet List No. 22) should be avoided when there is fulness, eructations of gas, or acidity. All foods which promote fermentation (Diet List No. 23) should be carefully avoided. A fomentation (10) should be applied over the stomach and abdomen at night, followed by a wet girdle (9) to be worn during the night, and the dry abdominal bandage (44) during the day, with nutritive treatment (page 331), electricity $(65,67,69)$, Swedish movements $(39)$, and subcarbonate or subnitrate of bismuth (62). In cases of prolapsed kidney, or in extreme cases in which acidity occurs within an hour or two after eating, especially when there is a suspicion of ulcer of the stomach, bicarbonate of soda (64) may be used in ten- to fifteen-grain doses before each meal. The Natural Abdominal Supporter (45) is required in cases in which relief is obtained by upward pressure with the hands applied to the lower part of the abdomen.

Flatulence.-This symptom indicates the presence of gas in the stomach or bowels, the character of which varies greatly, sometimes consisting of air which has been swallowed with the food in hasty eating. Sometimes, also, persons acquire the habit of swallowing air, as do horses, in which the habit is known as "cribbing." The act requires quite a strong effort, yet it is sometimes practiced constantly by hysterical patients, and sometimes also by dyspeptics to relieve 
unpleasant sensations at the stomach. A small quantity of air is swallowed in the act of gulping, which usually occurs just before vomiting, and can be accomplished at will by most persons.

The usual cause of gaseous distention of the bowels, however, is the formation of gas within the bowels themselves, by the fermentation of starch or sugar. Sometimes this occurs to an enormous extent, giving rise to belching and eructations, well compared to roaring in extreme cases. In some cases, accompanied by hysterical manifestations, the accumulation of gas will be almost instantaneous. In these cases the air may be swallowed, or regurgitated from the small intestine.

It is probable that flatulence, or distention of the stomach with gas, is sometimes due to the secretion of gas from the blood. It seems to be rapidly thrown out in great quantities in certain cases, particularly in nervous disease of the stomach.

When caused by fermentation, the gas usually consists chiefly of carbonic acid, from the destruction of starch and sugar, and is both tasteless and odorless. At other times it contains sulphureted hydrogen and other foul gases, from the decomposition of the albuminous and fatty elements of the food through the action of germs.

Intestinal flatulence, giving rise to rumbling in the bowels, or borborygmus, has the same causes as flatulence of the stomach. The form of flatulence which affects chiefly the small intestine seems to be in a great degree due to deficient secretion of bile. It affects chiefly weakly persons, those greatly debilitated by chronic 
rheumatism, especially, and more often women than men. It is frequently a cause of sleeplessness, and of unpleasant and erotic dreams, giving rise to nocturnal losses in persons whose sexual organs are in a weakened condition.

Flatulency of the colon, or large intestine, occasioning offensive gaseous discharges, arises from the decomposition of the retained contents of the bowel. It occurs most frequently as an accompaniment of constipation, and of a deficiency of bile. This condition is especially noticeable in cases in which a mixed or a meat diet has been used, in consequence of the decomposition of portions of undigested meat, which takes place in the colon. In cases of this kind there is constant poisoning of the system through the absorption of soluble poisons which are formed in connection with the offensive gases.

Treatment.-Temporary relief from flatulence may generally be obtained by sipping a little very hot water, either with or without a few drops of some volatile substance, as camphor, peppermint, or cinnamon. The hot water-bag over the stomach usually gives relief if there is much distention with the flatulence. Permanent relief from this condition can be obtained only by employing an aseptic dietary (Diet List No. 1). It is generally necessary to discard all articles difficult of digestion (Diet List No. 22), and those which are apt to promote fermentation (Diet List No. 23). In cases in which starch is digested only with difficulty, farinaceous foods (Diet List No. 4) must be avoided, and a nitrogenous dietary should be strictly adhered to for some time, with tonic water treatment (page 333), 
electricity $(65,66,68)$, massage $(30,31)$, Swedish movements (42), and daily exercise in the open air. Charcoal tablets $(\mathbf{4 7}, \mathbf{4 8}, \mathbf{4 9})$ should be taken after each meal.

Fermentation.--Septic and even putrefactive changes may take place within the body as well as outside of it. According to the conclusions arrived

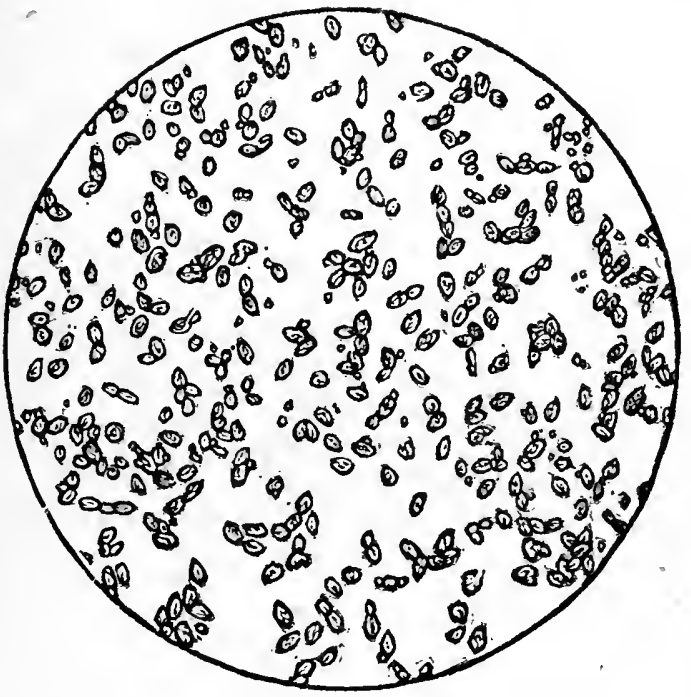

Fig. 17.-YEAST.

at by careful scientific investigation, the process of fermentation consists of changes in organized substances induced by germs received from the air. There are various kinds of germs, which induce different forms of fermentation. A variety of organisms known as yeast, excites the fermentation by means of which bread is raised, beer and wine fermented, etc. Through the 
action of yeast, sugar and starch are first converted into carbonic acid, alcohol, and water.

It is this kind of fermentation which produces that form of flatulence in which the stomach is distended with a tasteless and odorless gas. In the stomach, the process is precisely the same as in the baker's dongh trough or the beer vats of the brewer. When digestion is promptly performed, the food is digested and absorbed before it has time to ferment; but when it is slow, either on account of deficient muscular action on the part of the stomach, or because of deficient or defective secretion of gastric juice, fermentation takes place before digestion is completed. The conditions of the stomach are then peculiarly favorable for the occurrence of fermentation.

This change would always occur in the stomach were it not for the preservative influence of the gastric juice, which, when hydrochloric acid is present in sufticient quantity, effectually prevents fermentation by destroying the germs which enter through the mouth, or which are ordinarily taken in the food. As before remarked, however, if digestion is delayed for any reason, the germs taken in from the air or with the food quickly excite fermentative clianges, and gases and various poisons are dereloped.

Bacteriological experiments conducted in the Laboratory of Hygiene of the Battle Creek Sanitarium (Michigan, U. S. A.) for the purpose of studying the nature and action of germs found in the stomach, have shown that the healtly stomach is capable of destroying the germs which enter it in the act of eating, providing the food itself is sterilized ; that is, before being eaten 
it is heated to a temperature sufficiently high to destroy any germs which it may contain. This is found to be true, at least in the case of granose, a thoroughly sterilized food. In nine stomach fluids obtained after eating granose, germs were found in only two cases, and then very few in number.

Raised bread and ordinary biscuit always contain quantities of yeast germs in their interior which have not been exposed to a sufficiently high temperature to destroy their vitality. When taken into the stomach, these germs grow and develop, producing fermentation. Various yeasts, the spores of which are always to be found in flour, and which give rise to fermentation in the presence of warmth and moisture, oceasion in the stomach the alcoholic fermentation by which carbonic acid gas is produced in abundance, and even small quantities of alcohol. Other germs give rise to acetic acid, or vinegar fermentation, in which the alcohol produced by the action of yeast germs is converted into acetic acid. Other germs produce the lactic acid, or sour-milk fermentation, and still others give rise to a butyric-acid fermentation, the fermentative change which oceasions the rancid flavor of strong butter or decomposing fats.

All of these fermentations may take place at the same time. While the healthy stomach is able to destroy a few germs, the quantity of germs introduced with the food may be so great that even a healthy stomach cannot destroy them all. This is likely to be the case in the use of cheese, meat which has acquired a "high" flavor, sauerkraut, and such unnatural foods as oysters, either raw or imperfectly cooked, the liquor of which swarms with germs, infusoria, and other small 
creatures such as abound in the slime and ooze of stagnant waters along the seashore.

Treatment. - The treatment is essentially the same as that recommended for flatulence (see page 156). Special care must be taken to avoid all foods likely to promote fermentation (Diet List No. 23); charcoal tablets $(48,49)$ should be taken after each meal. In obstinate cases it is necessary to confine the diet to the very simplest articles. Zwieback or granose eaten dry will often be digested when fluid foods give great distress. A diet of granose and bromose (Diet List No. 25) will be found most efficient in relieving this condition in a great majority of cases.

In cases which do not yield to the above measures, lavage, or stomach washing (46), must be resorted to. At first it may be necessary to administer lavage two or three times a week, or even daily for a few days; but if the diet has been carefully regulated, the stomach washing may be made more and more infrequent, until, at the end of three or four weeks, its use nuay be dispensed with altogether. Too long-continued use of the stomach-tube is injurious.

Acidity.- This symptom may be due to the formation of either acetic or lactic acid in the stomach by means of germs, as has already been explained. The substances most likely to give rise to acidity are bakers' bread, milk, sweets, confectionery of all sorts, icecream, rich sauces, beer, wine, fluid foods, such as soups, and farinaceous foods when taken with large quantities of liquids, particularly cold fluids. In patients suffering from dilatation of the stomach, all 
starchy or saccharine foods are likely to give rise to acidity by reason of fermentation.

This condition requires the same treatment as has been recommended for flatulence and fermentation. The habit of relieving acidity by means of soda, magnesia, alkaline mineral waters, etc., is an exceedingly injurious one, and serves to aggravate the difficulty by lessening the activity of the gastric juice. The only remedy which can be properly used for relieving acidity is water. A copious draft of warm water may give relief, either by producing vomiting or by diluting the irritating acids to which the mucous membrane of the stomach is exposed.

Acidity from Hyperpepsia.-A form of acidity not due to fermentation is occasionally encountered. It is not due to the action of germs in the stomach, but to the formation of an excess of gastric juice or of gastric juice containing too much hydrochloric acid. This form of acidity is likely to be accompanied by intense burning at the pit of the stomach. Gastric crises, or attacks of severe pain in the stomach soon after eating, frequently occur in these cases. Hot liquids, acid foods, especially fruits, and in many cases sweet foods, increase the acidity, and are likely to give rise to pain. Eructations of a sour or bitter liquid are frequent in this form of acidity. There is not usually distention of the stomach with gas, or flatulence; but in some cases gas is formed, not by fermentation, but by secretion from the blood. In this form of acidity the pain usually begins half an hour or an hour after eating, and increases as long as the food remains in the stomach. 
In some extreme cases of this class the secretion of hydrochloric acid continues all the time, even when the stomach contains no food. In these instances, an extremely sour, acrid liquid is often regurgitated from the stomach before eating in the morning.

Treatment.-This condition is sometimes an exceedingly obstinate one, resisting the measures of treatment employed until both patient and physician are thoroughly discouraged. The dietary and general measures of treatment suggested for the relief of uneasiness at the stomach are applicable to cases in which acidity from excessive secretion of gastric juice is a prominent symptom. When these measures do not fully relieve the condition, subcarbonate or subnitrate of bismuth (62) may be taken before meals in twenty-grain doses, and bicarbonate of soda (64) either just before or soon after eating, or an hour or two later, as may be required.

Heartburn.-A burning or smarting sensation, often spoken of as "heartburn," is frequently felt at the pit of the stomach or the lower end of the esophagus, sometimes extending upward, and often accompanied by the rising into the mouth of a small quantity of a very acrid, irritating, pungent, foul-smelling liquid. The latest investigations on the subject seem to show that this symptom is due to the formation in the stomach of butyric or lactic acid, from the fermentation of some of the elements of food. The formation of butyric acid is particularly favored by the presence in the stomach of fatty substances. It is this acid which gives to old cheese and very rancid butter their peculiar pungency. It is also to its presence that the nauseating odor of vomited matters is due. 
The use of cheese, an article entirely unwholesome and utterly unfit to enter a human stomach, is very likely to produce the sensation of "heartburn."

Treatment. - When heartburn is habitual or a constant symptom after eating, lavage (46) is required. As a rule, however, simple regulation of the dictary is sufficient to effect a cure. The diet should be aseptic (Diet List No. 1). All foods difficult of digestion (Diet List No. 22) and likely to give rise to fermentation (Diet List No. 23) should be avoided. A dry dietary (Diet List No. 2), particularly the use of such foods as granose and zwieback (Diet List No. 25), and nut meal or nut butter in the place of butter, should be used. Tea and coffee and other drinks at meals should be avoided. Employ the general measures recommended for "Simple Dyspepsia," for which see page 245.

Water-Brash, or Pyrosis. - This is a symptom which commonly occurs when the stomach is empty of food, or nearly so; often before breakfast. A quantity of clear fluid, either slightly acid, alkaline, or neutral in taste, is thrown into the mouth from the stomach, varying in amount from less than a mouthful to half a pint or more. It is usually supposed to be caused by the accumulation of saliva in the stomach, which fails to absorb its fluid contents on account of inactivity. The expulsion of fluid may be accompanied by pain, or may be quite free from unpleasant sensations of any kind. It is a symptom said to be almost universal among the Lapps and New Zealanders, and is also exceedingly prevalent in Scotland, where it seems to be due to the use of insufficiently cooked oatmoal. 
Recent observations seem to show that the fluid expelled in water-brash is not raised from the stomach, but comes from the mouth only, being due to a sudden and profuse secretion of saliva by the salivary glands.

This symptom indicates an irritable condition of the sympathetic nerrous system. It may be produced by prolapse of the stomach, a floating kidney, pressure of waistbands, and similar canses, as well as by errors in diet; hence attention should be given to the removal of all these causes, as well as to the correction of the dietary. The treatment required is the same as that recommended for "Uneasiness at the Stomach," (see page 155$)$.

Nausea.-This symptom is a peculiar sensation referred to the stomach, which is not easily described, as it is unlike anything else. It usually precedes the act of romiting, though not always, and frequently exists without romiting. It is derived from the Greek word vaĩs, meaning "a ship," being always a prominent symptom in sea-sickness. Nausea indicates an irritation of the nerves of the stomach, which may be due to an excessive irritability of the nerves, whereby they are unusually excited by the mere contact of food with the mucous membrane of the stomach; or it may be the result of the irritation of the nerves of the stomach by acrid acids or poisonous substances produced by germs in the stomach or by the too long retention of food; or it may arise from the infection of the stomach by large quantities of microbes which the gastric juice, through some defect in quantity or quality, is not able to destroy. There are also some so-called nervous cases in which nausea is the result of the irritation of the vomit- 
ing centers in the brain by poisonous substances circulating in the blood.

Treatment.-Swallowing a few bits of ice, a few sips of hot water, ice over the stomach, ice about the throat, fomentations $(\mathbf{1 0}, \mathbf{1 1})$ over the stomach, fomentations to the spine, hot and cold applications to the spine (18), faradization (65) of the spine and stomach, gentle kneading of the stomach (31), subnitrate of bismuth (62) in ten- to twenty-grain doses, - these are simple measures which are sometimes successful in relieving vomiting. In cases of nausea in which there is reason to suspect offensive material in the stomach, lavage (46) should be employed, as the most efficient means of relieving this condition. In cases in which the nausea is habitual, except in cases of pregnancy, it is often advantageous to withhold all food from the stomach for a day or two, or even longer, nutrition being maintained by the nutritive enema (Diet List No. 24). Fasting is an excellent remedy for all conditions of the stomach in which there is excessive irritability. The general treatment should be the same as that recommended for " Uneasiness at the Stomach" (page 155).

Vomiting.-The nature of the act having been already described, only its indications need be noted. As a symptom of acute dyspepsia, it is very common, but is much less so in chronic disorders of the digestive organs. When present, it usually indicates something in the stomach which should be ejected, being an effort of nature to defend the system from injury. Sometimes the presence of simple mucus in excessive quantity, as in gastric catarrh, will give rise to vomiting. Vomiting also occurs very frequently in 
connection with whooping-cough, chronic bronchitis, and consumption, being due to violent coughing. It is also sometimes induced, as is coughing, by the tickling sensation produced in the throat by a relaxed or elongated palate. Tickling the throat with the finger or a feather will in many persons excite vomiting, especially if there is the slightest degree of nausea. Some persons can produce the act of vomiting at will.

When violent vomiting continues for a considerable length of time, the romited matter consists wholly of bile and mucus. The presence of bile leads many persons to suppose that bile is the canse of the vomiting, being an evidence of "biliousness," and having produced nausea by its presence in the stomach. This is usually an error. The bile is usually present in the stomach only as the result of reverse action, indicated by retching or long-continued vomiting.

The writer has, however, met several cases in which dilatation or prolapse of the stomach had dragged the pyloric orifice down to so low a point that the bile, which enters the small intestine a few inches below the lower orifice of the stomach, was led by gravity to - How into the stomach, instead of flowing in the opposite direction, as in health. In sucn cases bile is often found in the stomach when the organ is otherwise empty, and if not expelled by romiting, gives rise to constant nausea ; by nutralizing the gastric juce, it seriously interferes with stomach digestion. Such cases require replacement of the stomach and support of the organ in position by means of the abdominal'supporter (45). 
The violent efforts made in vomiting sometimes extend to the duodenum, where the bile exists naturally, causing it to be thrown upward into the stomach, whence it is expelled by vomiting. The first portions of bile vomited usually have a yellowish color, due to the fact that the alkaline bile is neutralized by the acid contents of the stomach. Afterward, when the bile becomes more than sufficient to neutralize the acid contents and secretions of the stomach, its natural greenish color appears.

Severe, protracted, and painful romiting is a common symptom of organic disease of the stomach, as in cancer, ulcer, dilatation, contraction, or some other structural change.

Treatment. - The measures suggested for the relief of nausea are also sometimes successful in the treatment of vomiting. However, it is not infrequently the case that vomiting continues in spite of the means mentioned. In such cases, lavage (46), or washing of the stomach, is the most important measure which can be employed, and it almost invariably succeeds, although not infrequently the passage of the tube must be repeated several times before the stomach becomes quiet. Surprisingly large quantities of fluid are sometimes removed in cases of this sort, especially in those commonly called "green vomiting." In these cases there is regurgitation of bile and other fluids from the intestine into the stomach.

Cases are occasionally encountered in which vomiting seems to be purely nervous in character. These are sometimes best relieved by the introduction of a small quantity of food into the stomach. 
There are still other cases in which romiting seems to be purely hysterical in character, and which can be controlled by commanding the patient in a very positive manner to cease romiting. The writer has cured a number of cases in this way. One young woman who had not retained a single meal for more than three years, ceased vomiting when convinced that she must do so or abstain from eating. I said to this young woman that I could not allow so much good food to be wasted, and that if she insisted upon vomiting everything she ate, she must cease eating. This she did for a short time, but was finally glad to come to terms, and agreed to vomit no more, which promise she kept in a very satisfactory manner.

Trentment.-Fasting, and the dietetic and other measures recommended for "Nausea" (page 166) and "Uneasiness at the Stomach" (page 155) should be employed in addition to the measures above suggested. The patient should rest in a horizontal position one or two hours after eating.

\section{Regurgitation-Rumination, or Merycism.} - Rumination is an act by which the food is returned to the mouth from the stomach by a reversal of the act by which food is swallowed, and without the violent efforts attending vomiting. In some animals, as the ox, sheep, goat, and others of the same species, all the food is returned to the mouth in this way, being rechewed. In man, regurgitation does not occur in health, being caused by an irritable state of the mucous membrane of the stomach, which excites contraction in the organ, and forces the food upward. Sometimes the act becomes more or less voluntary; but more 
often it is occasioned by a morbid condition of the stomach, and cannot be readily controlled by any effort of the will.

The ease with which some persons voluntarily regurgitate substances which have been swallowed is very curious. A woman who ruminated regularly, amused her friends and the public by swallowing two live gold fish, each about two and one-half inches long, regurgitating them alive twenty minutes afterward.

Regurgitation and rumination are perhaps only different degrees of a morbid condition. In regurgitation there is usually simply a return of a small quantity of liquid to the moutl. The patient complains of spitting up or tasting his food for some time after eating. In rumination proper, only the more solid portions of the food are returned to the mouth. In most cases of this kind the food is re-chewed and swallowed. Some patients who ruminate profess to experience a peculiar pleasure in re-chewing and regurgitating food. The old idea that rumination indicates some relation to ruminating animals, such as the sheep or cow, is of course without any foundation. It is a morbid condition, the exact nature of which is not well understood.

Treatment. - The food should consist of such substances as will be quickly dissolved in the stomach (Diet List No. 7). In severe cases, a liquid diet (Diet List No. 11) should be employed. Granose, bromose, and kumyss (Diet List No. 25) have been found especially helpful in extreme cases. The diet recommended for "Simple Dyspepsia" (page 245) should be employed.

Gripes, or Colic.-This symptom, sometimes called "belly-ache," is caused by irregular muscular" 
contraction, or spasm, of the small intestine. The exciting cause may be distention with gas, or the presence of irritating matters in the bowels. It is very common in patients subject to constipation.

Colic pains are sometimes caused by the introduction of air into the colon in the administration of the water enema. In using the bulb syringe, this is quite likely to occur, but it cannot take place to any extent in the use of the fountain syringe.

Treatment. - Fomentation $(\mathbf{1 0}, \mathbf{1 1})$, hot enema (22), drinking one or two glasses of hot water either with or withont the addition of a few drops of peppermint essence or camphor, with a dry or aseptic dietary (Diet Lists Nos. 1, 2) constitute the treatment for this disorder. In cases in which starch cannot be digested, a nitrogenous diet (Diet List No. 3) should be adopted, and all foods avoided which are difficult of digestion and liable to promote fermentation (Diet Lists Nos. 22, 23). The general treatment recommended elsewhere for simple dyspepsia or slow digestion should be employed.

Weight - Constriction - Tightness, etc.A symptom very often complained of by patients who may or may not have acidity, heartburn, waterbrash, and other local symptoms, is sometimes referred to as "a constriction," "a tightness," or "oppression," and is generaliy referred to a point a little to the left of the median line, just beneath the lower border of the ribs. It indicates a catarrhal condition of the membrane of the stomach. The sense of fulness which it often occasions after eating is deceptive, being quite as great in many cases when the stomach is empty, or when only a small quantity of solid food 
or liquid has been taken. Persons suffering thus are usually very despondent, and it may be this fact that has given rise to the term hypochondria, which literally has reference to the region in which the sensation described is felt. It often accompanies spermatorrhea and other forms of sexual weakness.

The "sinking sensation" at the stomach, of which many patients complain, though not identical with, is allied to, the sensation of weight described.

The sensations of weight, constriction, tightness, pulling, and others similar, when not the result of an excessire meal, are due to an abnormal sensibility of the nerves of the stomach. These sensations are most likely to be present in cases in which the stomach is prolapsed or dilated, and are due to a strain upon the branches of the sympathetic nerve which enters the stomach from the solar plexus.

Treatment. - The same general treatment should be employed as is recommended for "Uneasiness at the Stomach" (page 155.) The abdominal supporter is usually required (45).

Pain.-As a symptom of dyspepsia, pain may appear in the region of the stomach, or it may be referred to the spine, the chest, beneath the shoulderblade or between the shoulders, or, in fact, to any part of the body. The most usual pain is a dull aching after meals at the pit of the stomach, which is increased on pressure either with the palm of the hand or with the tip of the finger. In some cases pain, though not severe, is constant, being no greater after a meal than when the stomach is empty, and often being apparently relieved by bland food, but coming on again 
as soon as the stomach is empty. This sort of pain is usually accompanied by a pain beneath the shoulder. It indicates congestion of the mucous membrane of the stomach, and being usually preceded by the sense of weight already described, is doubtless indicative of gastric catarrh, in many instances at least.

Sometimes pain felt in the stomach comes wholly from increased sensibility of the mucous membrane. This condition is accompanied by abnormal sensibility elsewhere, in most cases, and commonly occurs in persons of hysterical tendencies, chiefly in young ladies. It not infrequently accompanies the condition rather raguely known as "spinal irritation." I have nearly always found tenderness at the epigastrium present in cases in which there was marked tenderness of the dorsal spine, and I have also noted that in these cases pain is always present at two important diagnostic points, known as Burkart's tender points, situated about two inches on each side of the umbilicus. The tenderness at these points is elicited by deep pressure, with the patient lying on his back and the knees drawn up.

A constant, wearing pain, though not severe, often becomes unbearable from its long continuance. It gives to a person a haggard, despairing look, which is also in part due to deprivation of sleep, another ill consequence of this variety of pain. The local pain is often aggrilvated by shooting pains emanating from the pit of the stomach, and running into the limbs and other parts of the body. Patients frequently complain of a pain felt "clear through the body," starting from the pit of the stomach, and terminating in a tender spot in the spine nearly opposite. 
Pain accompanied by vomiting of blood is indicative of gastric ulcer or of some other severe structural trouble. Flatulence produces a pain peculiar to itself. Neuralgia of the stomach is one of the most severe pains an individual can experience, often coming on suddenly, and in many cases soon after eating, and by its intensity not infrequently causing fainting.

Soreness on pressure, with neuralgic and other pains, also exists in the small intestines in some cases of dyspepsia, the conditions being essentially the same as those present in stomach pain.

It should be remarked in this connection that the various pains referred to are often mistaken for other diseases. The pain in the chest leads the patient and his friends to believe that he has consumption; and the emaciation occasioned by the defective nutrition seems to confirm this opinion. If the patient has a slight cough, this diagnosis is considered certain, and he is hurried off to Florida, California, Colorado; or some other locality supposed to be favorable for consumptives. By change of air, scenery, diet, increase of exercise, etc., the patient recovers, and the locality visited gets the credit of having cured a case of consumption, when the lungs have been sound from the first. If the patient stays at home, some quack or a worthless nostrum is quite likely to get the credit, and every real consumptive who hears of the wonderful cure forthwith tries the same remedy, but of course without benefit.

Such cases occur constantly, yet it must not be supposed that all pains in the chest come from the stomach. The same mistake is made in respect to other pains. In the region of the heart, it is supposed to be heart 
disease, especially if there is sympathetic palpitation of that organ! Pain between the shoulders is spinal disease. Occurring lower in the spine, it is thought to be kidney trouble, especially if there happens to be a sediment in the urine. Pain in the duodenum, occurring just beneath the lower border of the ribs on the right side, is "liver complaint," and is accordingly treated by a plaster or a "liver pad" !

It is often difficult to undeceive the patient, and to relieve him of the idea that he is suffering from some terrible organic malady, - an ulcerated lung, an abscess of the liver, incurable disease of the lieart or some other organ.

The superficial character of these pains, and the readiness with which pain is developed by pressure of the finger upon certain tender points, which for the most part lie between the ribs, along on either side of the sternum and the spine, and half-way between the sternum and the spine, is sufficient evidence that they do not arise from disease of the deep structures, or the organs which lie within the trunk. It should be remembered, however, that disease of the lungs, liver, or other internal organs may exist in connection with these superficial pains. Pains of the character described are generally due to irritation of the sympathetic nerve either from the presence of acrid substances in the stomach or from a strain upon the sympathetic nerves produced by prolapse of the bowels or stomach, or the constant pull upon these nerves by a floating or wandering kidney, a condition frequently associated with prolapse of the stomach, and the dyspeptic symptoms arising from this condition. 
Migraine, or nervous headache, is, in the writer's experience, always associated with dilatation of the stomach, and the formation within the stomach of poisons or poisonous substances, or the strain upon the sympathetic nerves caused by prolapse or dilatation of the stomach and associated organs.

Pain may exist in the stomach, liver, bowels, or other organs concerned in digestion, as the result of disease of the pneumogastric nerve - the nerve of sensation for these as well as the other organs of the abdomen.

Treatment. - The treatment consists of the hot-water or mustard fomentation $(\mathbf{1 0}, \mathbf{1 1})$, hot trunk pack $(\mathbf{7})$, wet girdle $(\mathbf{9})$, abdominal supporter $(\mathbf{4 5})$, and aseptic dietary (Diet List No. 1) or a dietary of well-disintegiated foods (Diet List No. 7). In extreme cases, a liquid diet (Diet List No. 11), the use of granose, bromose, and kumyzoon (Diet List No. 2.5), electricity to the spine, stomach, and abdomen $(65,67,69)$, and the general treatment recommended elsewhere for " $\mathrm{Un}$ easiness at the Stomach" (page 155) should be employed.

Biliousness.- What is termed "bitiousness," or "a bilious attack," is really acute gastric catarrh. From the long retention of undigested food, the stomach becomes irritated to such a degree that nausea and vomiting are produced. At first the matters vomited consist of undigested food in an advanced stage of decomposition, as indicated by the foul odor and nauseous taste. After vomiting has continued for some time, bile and mucus are the principal matters expelled. The patient soon feels better, and in a few days is as well as ever. In these cases the difficulty is really in the stomach, and not in the liver, as many suppose, though such 
attacks may be accompanied by disturbance of the functions of the liver. The irritation extends down to the duodenum, its mucous membrane becoming so swollen that the opening of the duct from the liver is obstructed. This occasions retention of the bile, and absorption takes place, when the individual notices a jaundiced color of the skin, a dingy appearance of the white of the eye, etc. This is what gives to this symptom, or group of symptoms, the term biliousness, when it is really gastric catarrh.

One of the most common causes of acute gastric catarrh is "taking cold" after overeating or eating food difficult of digestion.

Biliousness is due to the abnormal development of poisons in the stomach or intestines, and the failure of . the liver to destroy these poisons or remove them from the blood. A healthy liver is able to protect the body from an ordinary amount of poisonous substances; but when a person indulges in such gross articles of diet as cheese, raw oysters, rich gravies, fat meats, or habitually makes free use of meats of any sort, or paralyzes his stomach by the use of alcohol, tobacco, tea, or coffee, and in cases in which the stomach retains its food for too long a time as the result of dilatation or prolapse from corset wearing, the poor liver is overwhelmed with such a quantity of poisons that it is unable to complctely disinfect the blood brought to it from the stomach and intestine; consequently the whole system is invaded by them, and a condition of general systemic poisoning, or what Professor Bouchard terms "autointoxication," or self-poisoning, is produced. It will readily be seen that biliousness is not dne to 
torpidity of the liver, but to abuse of the liver. The liver does its work as well as it can under the circumstances. The thick brown coat upon the tongue and the bad taste in the mouth experienced by bilious persons are indicative of an unclean state of the whole alimentary canal and the general poisoning of the entire body. It is not simply the patient's tongue which tastes bad, but it is he himself. The relief obtained by the use of mercury and other so-called cholagogues is not due to the stimulation of the liver, as is generally supposed, but rather to the disinfection of the alimentary canal through the carrying off of a quantity of germs and decaying substances.

The treatment of this condition should be the same as is elsewhere recommended for "Bilious Dyspepsia" (seé page 251).

Appearance of the Tongue.- While the importance of the condition of the tongue as a symptom of dyspepsia is such as to make its mention necessary, it should be borne in mind that its indications have more distinct reference to the system in general than to the stomach in particular. A clean tongue, of natural color, appearance, and moisture, is a pretty sure indication of health. Unnatural redness of the tongue, most commonly at the tip and edges, sometimes with a strawberry appearance, indicates an irritable state of the stomach. It is usually accompanied by soreness at the pit of the stomach, little appetite, and great thirst.

A yellowish or creamy coating on the tongue indicates an inactive state of the stomach and intestines, giving rise to a foul condition of the organs. If a 
small portion of the slimy yellow or brownish material covering the tongue is planted upon the cut surface of a boiled potato, and exposed in a moist room for a few days, the potato will be found covered with a thick growth of germs closely resembling that upon the tongue, demonstrating that the coat upon the tongue is really a growth of fungi similar to a patch of mold upon the wall.or mildew upon a garment. When this condition exists, not only the tongue, but the stomach, is also infested with these mischief-making microbes. The bad taste experienced in connection with a foul tongue is due to the production of poisons by the germs. The headache, nausea, loss of appetite, and other symptoms which often accompany a coated tongue, and the bad. taste in the mouth, are due to the absorption of these poisons from the stomach, where they are often produced in enormous quantities. Plate $V$ shows the appearance of germs from the tongue and the stomach as seen growing upon the cut surface of a sterilized potato, and also the appearance of these germs as seen under the microscope.

A white coat on the tongue indicates a feverish condition. This often appears in connection with unnatulaal redness at the edges and tip, indicating irritation.

A broad, pale, flabby tongue indicates a weakened, debilitated condition of the digestion and of the whole system. A tongue of this sort usually exhibits indentations on its margin, formed by the teeth, owing to its flabby state, usually present in hypopepsia.

A brown coat upon the tongue is very common in acute dyspepsia. It is often accompanied by a bad taste in the mouth, with unnatural dryness. Sometimes 


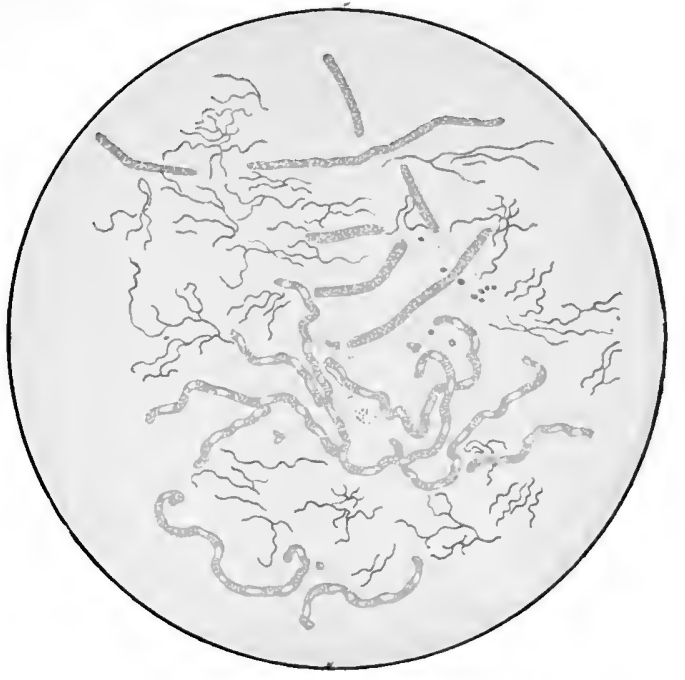

Fig. 1.- Mouth Germs Found upon the Surface of a Coated Tongue, greatly magnified.

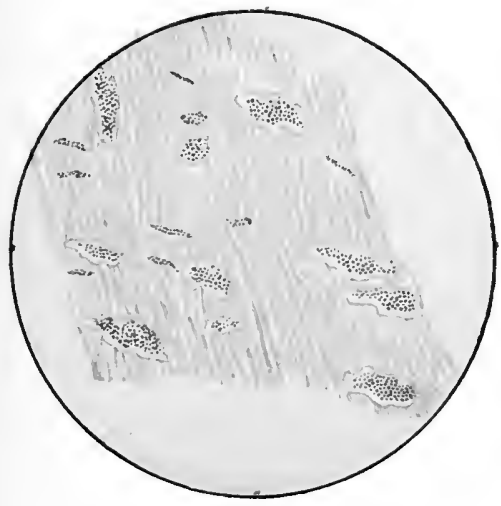

Fig. 2.- Germs which Cause Decay of the Teeth.

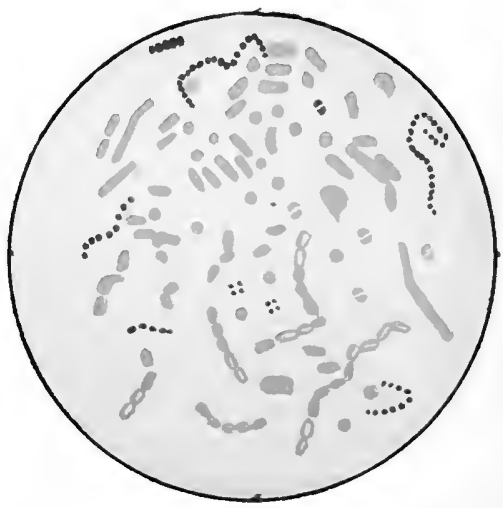

F1G. 3.- Germs found in the Stomach Fluid after a Test Meal, greatly magnified. 

this is due to sleeping with the mouth open, by which the secretions and epithelium become dried to an unnatural extent.

Sometimes the tongue is coated in the middle, the edges being smooth and clean. This is due to the fact that the epithelium has become softened on the smooth portions, and has been rubbed off by the friction of the teeth. Clean spots are also due to lost epithelium, in many cases.

Transverse fissures of the tongue indicate a diseased state of the mucous membrane of the stomach, usually due to acid dyspepsia.

Treatment. - A yellow or brown coat upon the tongue indicates an infected condition of the stomach, which requires the treatment recommended for "Bilious Dyspepsia" (page 251). A broad or pale, flabby tongue indicates hypopepsia, and requires the treatment recommended for this condition (see page 260). In cases accompanied by rise of temperature, the patient's diet should bo carefully restricted to such foods as are suitable in fever (Diet List No. 12). Fine charcoal $(47,48)$ should be freely used in cases in which the tongue is unusually foul, and the patient should be made to drink water freely. The amount taken in twenty-four hours should usually be from three to six pints More is often advantageous.

Aphtha.-In cases of acidity of the stomach, occasioning irritability of the organ, little pimples often appear on the sides and under part of the tongue, and just within the corners of the mouth. After a short time, small white patches of lymph may be seen, which frequently leave very troublesome little ulcers when 
they disappear. These very annoying ulcers are positive evidence of stomach disorder, though the patient will often assert that he has no difficulty with his food, and never experiences any pain or inconvenience connected with the stomach.

Aphthæ are probably most often present in cases of hyperpepsia, or excessive secretion of the gastric juice; but the writer has also found them present in hypopepsia.

Treatment.- The mouth should be thoroughly cleansed after each meal; it should be rinsed every hour with a saturated solution of boric acid or chlorate of potash. The ulcers may be touched with a solution - forty grains to the ounce - of nitrate of silver, after being carefully dried. Treatment for either hyperpepsia or hypopepsia should be employed, according to the indications of other symptoms.

“Throat-Ail." - Congestion of the pharynx, with a granular condition of the mucous membrane, relaxation and elongation of the uvula, accompanied by a sense of constriction, tightness, dryness, tickling, and other symptoms, are not infrequent indications of stomach. disorder. The patient is often much troubled with a tenacious mucus which he finds difficulty in dislodging. Sometimes the throat trouble amounts to real difficulty in swallowing.

What is known as "clergyman's sore throat" is usually associated with disorders of digestion.

Treatment - Fomentations $(\mathbf{1 0}, \mathbf{1 1})$ applied to the throat at night, and followed by a cold compress, well covered, to be worn during the night, and a cold sponge bath (1) with vigorous rubbing in the morning, are ex- 
cellent remedies for hoarseness. Gargling hot water in the throat three or four times a day is also useful. The vaporizer $(\mathbf{5 9}, \mathbf{6 0})$ should be used five or six times a day, five to ten minutes each time. It may be used to advantage even more frequently, if convenient. The course of tonic treatment and the general measures indicated for slow digestion should be employed.

Sour Taste in the Mouth.-This symptom, not a very infrequent one, is due to an acid condition of the saliva, which not only renders it of little use as a digestive fluid, but also ruins the teeth, by destroying the enamel.

In anomalous cases the saliva lias a sweetish taste.

In inactive conditions of the liver, it is likely to be bitter, so that the patient complains constantly of a bitter taste in the mouth. Viscidity of the saliva, accompanied by an unpleasant sense of heat in the mouth in the morning, is a common indication of derangement of digestion.

- In the careful examination of the saliva in a large number of eases, I have found acidity present much more often than I had formerly suspected. A careful examination of the condition of the digestive organs must hence include an investigation of the condition of the saliva as regards its chemical reaction and its digestive activity.

Treatment. - Rinse the mouth with a solution of carbonate of soda (64) three or four times daily; cleanse the mouth thoroughly before and after each meal with an antiseptic dentifrice (57); or rinse the mouth with cinnamon water (58), cleansing by ordinary means. The diet and other treatment should be such as is recom- 
mended for "Simple Dyspepsia" with fermentation (see page 245).

Constipation.-Though often an accompaniment of dyspepsia, this condition is not a constant one in that disease. There seems to be a quite general error on this point, since many patients confound the use of the term indigestion with constipation. I have often been told by patients, in answer to an inquiry respecting the state of the bowels, that their "digestion" was very regular, or very irregular, as the state of the bowels happened to be. A person may have very poor stomach digestion without any marked disturbance of the bowels; yet constipation, or costiveness,- some make a distinction between the two, - is very rarely present without disorder of some sort in the digestive apparatus.

As a rule, it may be expected that any disturbance in the function of those digestive organs which lie below the stomach are secondary to disease of the stomach. Intestinal eatarrh follows gastric catarrh; cirrhosis of the liver, jaundice, gall-stones, and other hepatic affections are also eonsequences of chronic gastrie or gastro-intestinal eatarrh. Biliousness also begins in the stomach, as has been shown. Cancer of the liver is nearly always secondary to disease of the stomach.

In constipation there may be either excessive hardness of the fecal matters through too long retention, and consequently too great absorption of the fluid portion, or there may be loss of nerve sensibility at the lower, or anal, orifice of the intestinal canal. 
Treatment.-For the treatment of this condition, see page 279 .

Diarrhea.-This also is as marked a symptom of dyspeptic conditions as the preceding, though less frequent. Whether constipation or looseness is present depends on the particular form of indigestion. When the most prominent difficulty is slow digestion, constipation is usually present. In bilious dyspepsia, the decomposition of the food gives rise to such a degree of irritation that diarrhea is induced. Diarrhea in these cases is doubtless the result of the effect of poisonous substances generated by the germs developing in the alimentary canal, and which the digestive fluids have failed to destroy.

Diarrhea is also present in cases in which there is so great irritability of the mucous membrane that the food is hastened along without complete digestion. Not infrequently the two conditions constipation and diarrhea, alternate, each being occasioned by the other, the vital forces being too weak to maintain a healthy medium of activity. Hardened fecal matters are retained until they become a source of irritation, then diarrhea ensues until the bowels have been emptied, in part at least, this excessive activity being followed by a return of the constipation.

Treatment. - The treatment of this symptom will vary according to the conditions which gave rise to it. If other symptoms indicate foul or bilious dyspepsia, then the treatment elsewhere recommended for this condition should be employed, together with a large hot enema administered daily. Chaircoal $(47,48)$ or 
antiseptic charcoal tablets (49) should be habitually used.

When diarrhea is due to excessive irritability of the mucous membrane of the stomach, indicated by a red, sensitive tongue and other symptoms of irritation of the alimentary canal, liquid food (Diet List No. 11) or food which will readily dissolve in the stomach (Diet List No. 7) should be used. A diet of kumyzoon (Diet List No. 25), kumyss, or buttermilk is sometimes required. An enema taken after each movement to cleanse the bowels, fomentations (10), a wet girdle (9), and in cases of prolapse of the bowels, with dragging sensation across the abdomen, the abdominal supporter, are very helpful measures used in connection with those means which are indicated by other symptoms of indigestion. In old and very feeble patients, "Nutritive Treatment" (page 331), and in stronger subjects, "Tonic Treatment" (page 333) should be employed.

Long, patient, and persevering treatment is required in these cases. Sometimes in chronic diarrhea, the "Rest-Cure" (see page 329) for two or three weeks, and a diet eonsisting chiefly of kumyss, kumyzoon, or buttermilk, and eggs is neeessary to give the patient a good start in the direction of health. The daily hot enema (22) and "General Tonic Treatment" (page 333) are required in these eases.

Backache. - This symptom is so common in dyspepsia that it requires separate consideration. A chronic ache in the lower part of the back is due to an irritation of the lumbar ganglia of the sympathetic nerve (page 193). Pain or soreness between the shoulders is due to irritation of the solar plexus. The symptoms 
arise most frequently from prolapse and dragging of the stomach and bowels. Prolapse of one or both kidneys or of the liver or spleen is also frequently present in these cases: Examination of the abdomen shows a sagging or abnormal protrusion of the abdomen at its lower portion. In these cases tenderness is found on making deep pressure at points two inches on either side of the umbilicus. Constipation is usually present.

Treatment. - The following measures will be found the most effective : "General Tonic Treatment" (page $333)$, fomentations $(\mathbf{1 0}, \mathbf{1 1})$ to the spine, hot and cold to the spine (18), hot and cold trunk pack $(\boldsymbol{8})$, applications of electricity to the spine $(\mathbf{6 5}, 6 \mathbf{6 7}, 69)$, Swedish movements (41). Proper measures for the relief of constipation should be employed (see page 279).

The Stools.-Quite too little attention is paid to the character of the bowel discharges by physicians as well as by patients. In all cases of dyspepsia, they should be carefully and frequently examined. The stools should be well-formed and of moderate consistency. Ragged, unformed stools indicate some degree of irritability of the digestive tract. Watery stools show deficient absorption, or too profuse secretion from the mucous membrane. Slimy discharges indicate a catarrhal condition. Small, pellet-like masses are indicative of constipation due to deranged function of the colon. If the discharges are unnaturally light in color, too little bile is present. An unnaturally dark color, with very offensive odor, often accompanies foul dyspepsia. The appearance of portions of undigested food indicates great inactivity of the digestive organs. 
A microscopic examination is often extremely useful, as by this means it can be ascertained, in many cases, what particular element of the food may not be well digested. For example, if fat globules are found in great abundance, the indigestion of fat or oleaginous substances is indicated ; if portions of undigested meatare discovered, difficulty in digesting animal food will be understood.

Examination of the fecal discharges also affords the only reliable evidence of worms, as by this means eggs, if not the worms themselves, may certainly be found if they are present. Examination for the eggs of parasites requires the use of the microscope in the hands of all expert.

Trentment. - The treatment required for abnormal conditions of the fecal matters varies according to the indications. Dark, offensive fecal matters indicate a foul condition of the alimentary canal, requiring an aseptic dietary (Diet List No. 1), eharcoal or antiseptic charcoal tablets $(\mathbf{4 7}, \mathbf{4 8}, \mathbf{4 9})$, and the general measures recommended for bilious dyspepsia. Bilious or septic indigestion (page 251), with slight discharges, indicates a catarrhal condition of the bowels. The presence of mucus in excessive quantity indicates a catarrhal condition of the intestines. If the mucus is discharged first, a catarrhal condition of the lower portion of the rectum exists. If the mucus follows the fecal discharge, the catarrhal condition is located higher up in the bowel. If the mucus is mixed throughout the fecal mass, it is probable that a considerable portion of the colon is affeeted. This condition requires an aseptic dietary (Diet List No. 1), especially the disuse of all forms of flesh 
food, the use of charcoal $(47,48,49)$, a large hot enema daily, to thoroughly cleanse the bowels, to be followed by an astringent enema consisting of a dram of tamnin to a quart of water, the last to be retained for half an hour, if possible. Masses of undigested food indicate the need of a dietary consisting of welldisintegrated food substances (Diet List No. 7).

For a liquid dietary (Diet List No. 11), in extreme cases, a diet of kumyzoon (Diet List No. 25), kumyss, or buttermilk is required.

The Urine.-Examination of the urine is important, though little need be said. on this point, as a reliable examination can be made only by a competent physician. A white, pink, reddish, or brick-dust sediment is very common in dyspepsia, together with other deposits. These are most often found after an unusually severe attack of indigestion. The fact that deposits in the urine are frequent in this disease, is mentioned more particularly because those who are ignorant on the subject are often led, by the advice of quacks or otlierwise, to take this as evidence of disease of the kidneys, of which it is not a positive indication. These deposits are the result of the inability of

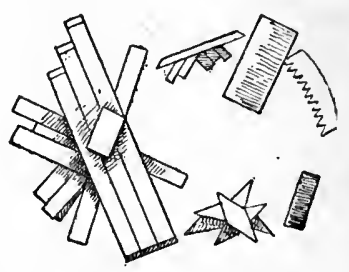

Fig. 18.-URIC ACID Crystals. the liver to oxidize, or reduce to urea, the great quantity of imperfectly prepared matter thrown into the blood. They are particularly liable to be present in persons who use considerable meat. (See Fig. 18.)

Treatment. - A white, pinkish, reddish, or brickdust sediment indicates the necessity for an aseptic diet 
(Diet List No. 1), and especially the aroidance of all articles hard to digest and likely to undergo fermentation (Diet Lists Nos. 22, 23), and the disuse of meat.

This condition often precedes Bright's disease, and should receive careful attention. The patient should drink from three to six pints of water daily, and should exereise ont of doors two or three hours daily, the exercise being as vigorous as is consistent with the strength of the patient. A hot bath of some kind once or twice a week, and "General Tonic Treatment" (see page 333) should be employed. Fomentations (11) over the liver daily, and the wet girdle $(\mathbf{9})$ are also measures of value.

Dryness of the Skin.-A peculiar dry, rough condition of the skin is very common in dyspepsia, though not peculiar to the disease. In these cases, the natural secretion of the skin is greatly diminished. The patient will frequently complain that he never sweats.

In some forms of dyspepsia, the opposite condition is present, the skin being tawny, and having a greasy feeling. Occasionally it is unusually clear, pliable, and sensitive, sometimes being almost transparent. These cases usually oceur among nervous dyspeptics.

Treatment._-"General Tonic Treatment" and a daily oil rub (27) are indicated for the relief of this condition.

Skin Eruptions. - The use of particular articles of food will in certain individuals give rise to various skin eruptions soon after eating, the eruption being accompanied by marked evidences of indigestion. Eczema, acne, and other skin diseases are chiefly dependent upon disordered digestion. All the glandu- 
lar structures of the body are controlled by the sympathetic nervous system. In diseases of the stomach, this nerve is certain to be more or less affected, and hence disturbance arises in the glands as well as in the other structures under the control of the sympathetic nerves. Indigestion is in this way the principal cause of acne, or pimples of the face.

Treatment. - Relief of the digestive disorder by "General Tonic Treatment" (see page 333), should first be sought. Zinc ointment, obtainable from any drug-store, may be applied to the eruptions. For chronic cases of eczema, with a thickening of the skin, the daily application of hot water by moist cloths $(\mathbf{1 0})$ or a hot pour (19) is a proper measure to be employed. Acne sometimes requires the use of a hot solution of corrosive sublimate, one part of the sublimate to two hundred of water, after a thorough cleansing of the parts with soap and water. Care should be taken not to get the solution into the eyes or mouth, as it is very poisonous. Use daily for five minutes.

Cold Feet, etc. - To cure cold feet, do not heat them at the fire nor parboil them in hot water; this only relaxes and weakens the circulation. A better plan is this : Just before going to bed, place the feet in a cold bath, with the water about one fourth of an inch deep. In a few minutes, dry and rub warm with a coarse cloth or with the hand. The alternate hot and cold foot bath may be used with still greater effectiveness. At night, if the feet cannot otherwise be kept warm, hot jugs or foot-stones may be employed; but it is far better to be independent of such artificial substitutes for vital heat by wearing warm bed socks. 
When the feet or hands are too hot, they may be cooled by employing cold instead of heat. Ice-water may be employed when necessary, and will be found a most excellent means of relieving the unpleasant burning of the feet from which many people, especially older persons, often suffer much.

Coldness of the extremities in dyspeptics is due to an irritated condition of the abdominal sympathetic nerve, whereby the blood-vessels are caused to contract, so that an insufficient amount of blood is circulated through the extremities. Patients suffering in this way often complain of a dragging sensation across the lower abdomen, or an aching in the back, especially when obliged to be much upon the feet. There is also a disposition to elevate the feet in sitting, and a symptom commonly known as "fidgets" is often present. These symptoms indicate the necessity for relief of the irritation of the abdominal sympathetic, and also for the support of the abdominal organs, the downward displacement of which is a cause of the irritation. The principal measures required are fomentations to the abdomen daily, the wet girdle $(\mathbf{9})$, and "General Tonic Treatment". (see page 333 ). The abdominal supporter (45) should be worn constantly when the patient is exercising in an upright position. The application of electricity $(65,66,67)$ and Swedish movements (40) are also very useful measures in these cases.

Headache. - Various forms of headache are among the most common symptoms of dyspepsia. In different cases, different parts of the head are affected, and in the same person at different times. The whole 
head may be affected, feeling, as the patient sometimes says, "as though it would burst." Again, the pain may be confined to the temples, or to the back or front of the head, to the top of the head, to the region of the ears, to the eyeballs, to the upper part of the neck, or it may extend down between the shoulders. In many cases there is also tenderness of the part affected.

When headache occurs during digestion, or soon after eating, it is usually of a dull, heavy character, often located in the front part of the head, and is accompanied by more or less confusion of thought, cold feet, and sometimes dimness of vision. This form of headache is often accompanied by a sense of weight at the pit of the stomach.

The headache which comes on the next day or several hours after taking an indigestible meal, is of a more severe character. It is commonly accompanied by pain and tenderness just below the ribs on the right side, in the region of the duodenum, which is probably the part chiefly affected.

Migraine, or Nervous Headache.- This affection, long treated as a nervous disease, has been clearly proved to be a purely symptomatic malady, resulting from irritation of the sympathetic nerve as the result of the action of poisons formed in the stomach. A careful study of a large number of these cases has convinced the writer that nervous headache occurs only in persons suffering from dilatation or prolapse of the stomach. After having made a careful examination of a large number of cases of this disease, numbering more than one hundred in all, I am able to say that I have failed to find a single case in which there was not 
evidence of great irritation of the abdominal sympathetic nerve, as shown by extreme sensitiveness at the tender points first pointed out by Burkart, in 1882 . These points lie about two inches on each side of the umbilicus, at the posterior surface of the abdominal cavity. An indication of chronic poisoning which is almost invariably to be found in these cases is the foul tongue and malodorous breath presented by the patient, and which afford the most indubitable evidence of a septie condition of the alimentary canal. A peculiar bitter, or what is frequently described by the patient as a " nasty," taste in the mouth nearly always accompanies this disease. The disagreeable, and often fetid, odor of the breath is evidently derived from the formation of sulphuretic compounds, the extremely toxic character of which is well known. Pepper mentions cases in which jewelry worn by the patient was always tarnished during an attack of this disease.

Intellectual overwork or worry is an exeiting cause of the disease only through the fact that by reflex action downward it increases the sensitive condition of the abdominal sympathetic, and at the same time retards the digestion, and so favors both the production of toxins, which are the direct cause of the disease, and the susceptibility of the sympathetic nerve to the action of these poisonons substances.

The frequent occurrence of attacks of migraine at the menstrual period in women is occasioned simply by the increased sensitiveness of the nervous system at that time. It is a well-established fact that both mental and nerrous disturbances are much more likely to occur in women at the menstrual period than at other times. 
Those who have made a careful study of this disease will at once recall many circumstances which are confirmatory of these views. Many have doubtless observed in certain cases a peculiarity of the disease mentioned by Niemeyer, who remarks as follows: "In some cases, a severe attack, particularly if it ends with sick stomach, results in a certain immunity to relapses." Vomiting is, of course, in some degree equivalent to a lavage, and so affords the patient temporary exemption from suffering. The fact that great benefit may be derived from the use of saline laxatives in many cases, the disease being frequently aborted by the early use of an active cathartic, is another confirmatory evidence. It will also be remembered that food of any kind, no matter how simple or digestible, frequently aggravates the disease, although there are occasional cases, as mentioned by Brinton, in which the taking of a hearty meal seems to terminate an attack, probably by exciting stomachic and peristaltic activity. Niemeyer remarks as follows with reference to the treatment of this disease :- -

"In most cases we can do nothing but attend to existing disturbances of the general health and of the digestion, and the chances of benefit from treatment are much greater when we can discover any such disturbances. During the attack we should spare the patient from the use of any remedies, and let him take nothing but water."

Viewed from this standpoint, migraine is no longer a complex or mysterious nervous malady, but simply a state of systemic poisoning, the origin of which is decomposition of food products in a dilated or 
prolapsed stomach which, through muscular weakness, is unable to rid itself of its contents with sufficient promptness to prevent septic and putrefying processes. Although these causes may be constantly in operation, they do not usually produce pronounced symptoms of poisoning except at intervals, the kidneys ordinarily eliminating the poisonous substances with sufficient rapidity to prevent those "nerve storms" to which the term migraine, or megrim, has been attached, except when the nervous system is by excessive nervous or mental activity, or by the functional disturbance of menstruation, rendered unusually susceptible.

The remedy for this malady is to be sought, then, not in the discovery of some new drug which shall temporarily paralyze the nerrous sensibility, reduce the blood pressure or raise it, as may be required in the individual case, but in the removal of the real cause of the disease. The writer believes that every case of migraine is capable of being radically cured.

Treatment. - The treatment required in these cases consists of two classes of measures :-

1. Those that are palliative, or useful during an attack.

2. Those that are curative, to be used in the intervals.

The most efficient of all palliative means is lavage, or washing out of the stomach. By employing lavage (46) at the very beginning of the attack, it may often be cut short, and by adopting this measure still earlier, as on the appearance of the first premonitory symptoms, it is found possible in many cases to prevent the attack altogether. Many times the stomach-tube brings up a 
great quantity of ill-smelling, undigested, and decomposing food. In other cases, no food, but a small quantity of clear but very bitter and acrid fluid, is obtained. When there is very marked dilatation, it is sometimes necessary to repeat the lavage a number of times before the stomach is completely cleared of its fetid contents. Not infrequently there is bile present. It should not be forgotten that the bowels as well as the stomach may contain a considerable amount of decomposing matter; hence a large hot enema (22) should be administered as a means of cleansing the lower portion of the alimentary canal. This should be done whether the bowels are known to be constipated or not. The enema serves a useful purpose, not only in washing out the bowels, but in supplying fluid to the body, by which the kidneys are aided in eliminating the irritating poisonous substances with which the blood is charged.

Hot and cold sponging of the spine (18), fomentations to the head when the face shows pallor, and also to the stomach, heat to the extremities if cold, and applications of galvanic and faradic electricity $(65,70)$, are needed. It must be admitted, however, that no remedies are of very great value when an attack is once fairly started, although faithful use of the measures suggested will eertainly serve to eut short the attack. The use of antipyrin, migranin, antifebrin, and other allied drugs is to be deprecated, for the reason that these drugs, though sometimes temporarily palliative, soon lose their effect, requiring an increase of dose at each successive attack, until the injurious results of the drug may become a more serious matter than the disease itself. 
The patient should not eat during an attack, or at least not until after the first twenty-four hours. There is, however, an occasional exception to this rule, in which eating after the omission of a single meal seems to aid in bringing the attack to a termination by unloading the contents of the stomach into the intestine, and thus provoking movement of the bowels. The first food taken should consist of some simple cereal or fruit preparation. All forms of flesh foods and animal extracts, and also milk, should be aroided. Tea and coffee, which are popular remedies for sick-headache, are highly detrimental ; for although they may sometimes mitigate the symptoms during an attack, they prepare the way for a speedy recurrence of another. Granolil, granose, zwieback, gluten, and water breads of various kinds ; grapes, peaches, in fact, fruits of all kinds; and such green regetables as peas, asparagus, and string beans, are wholesome and suitable, but for these cases should be cooked without milk or cream. Nuts, if well ehewed, - preferably, however, taken in the form of nut meal (Diet List No. 25),- afford the most suitable form of fat. Milk is allowable only in the form of buttermilk, kumyss, kumyzoon (Diet List No. 25), or cottage cheese. It should be carefully sterilized.

The curative treatment of migraine consists of a careful adherence to an aseptic dietary (Diet List No. 1), special preference being given to the articles mentioned. Everything possible should be done to build up the patient's general health. For this purpose the cool morning sponge bath (1), followed by vigorous rubbing with a towel or flesh-brush, and the wearing of 
the moist abdominal bandage (9) during the night, are especially helpful. Outdoor exercise should be taken daily to the extent of producing gentle perspiration. Bicycle riding is especially helpful, also boating and horseback riding. Patients who suffer from dilatation and prolapse of the stomach, however, should take care to support the prolapsed viscera by a proper abdominal supporter. For this purpose the author has devised an instrument called the Natural Abdominal Supporter (45) which elevates the viscera in a manner exactly similar to that in which persons often support themselves with their hands when they have an unpleasant dragging 'sensation in the lower abdomen. Tenderness of the abdominal sympathetic may be relieved by the daily application of the hot water-bag for an hour or two, or a fomentation (10). Special pains should be taken to masticate the food very thoroughly; and in cases of extreme dilatation of the stomach, the almost exclusive use of dry food (Diet List No. 2) and well-disintegrated foods (Diet List No. 7 ) is required, for a few weeks at least.

Lastly may be mentioned the use of an intestinal antiseptic as a means of preventing the development of germs and the formation of the poisons which are the real cause of this disease. One of the very best of these is vegetable charcoal, preferably charcoal made from cereals $(47,48)$. The addition of a small proportion of sulphur increases the efficiency of the charcoal. The writer has for many years employed a mixture of this sort, which is manufactured by the Modern Medicine Company, Battle Creek, Mich. (U. S. A.), and put up in the convenient form of very 
palatable tablets (49). Two or three of these taken after each meal aid the stomach in preventing the action of germs, and at the same time so act upon the bowels as to prevent constipation. I know of no remedy so valuable in the treatment of this class of cases, and, in fact, all cases arising from septic or foul conditions of the stomach and bowels.

Regularity of the bowels should be established by the use of coarse food like granose (Dict List No. 25) and other whole-grain preparations, and by the use of the graduated encma, and other measures suggested elsewhere in this work (see page 279). Lavage should be employed once or twice a week in cases of extreme dilatation of the stomach, or when fermentation or considerable quantities of mucus are found present by an examination of the stomach fluid. The charcoal tablets (49) and larage should be employed until the tongue becomes clean.

Swedish movements (40), massage of the stomach and abdomen $(29,31)$, daily replacement of the abdominal viscera $(32,33)$, with general massage (36), are measures of great value in cases of migraine.

Nervous Apoplexy.- This is another condition dependent upon a disturbance of the abdominal sympathetic, the symptoms of which so closely resemble those of apoplexy that the person suffering from it sometimes lives in almost constant dread of death from paralysis as a result of cerebral apoplexy. The subjects of nervous apoplexy complain more or less habitually of giddiness, especially after eating, double vision, scintillations before the eyes, sudden loss of vision, sudden change of color in surrounding objects, 
everything appearing green, blue, or red, as the case may be, and occasionally sudden loss of consciousness. The writer has had several cases under his care in which these symptoms were very pronounced, the patients often being brought in from the porches or corridors, where suddenly, in the midst of a conversation, they had fallen to the floor insensible. Attacks of nervous apoplexy are frequently induced by fright or some sudden, strong emotion, or even by a loud sound.

Treatment.-In the treatment of these cases I have sometimes seen the most remarkable results follow the application of the abdominal supporter (Fig. 40 ), whereby the strained and irritated sympathetic nerves, which are always extremely sensitive in these cases, were afforded relief by the support of the prolapsed stomach, bowels, or other viscera. The moist abdominal bandage worn at night, fomentations (10) to the spine in the morning, followed by a cool sponge bath (1), massage (31, 36), Swedish movements (41), and, if possible, the application of galvanic electricity to the spine and abdomen $(69)$, are the most efficient measures of treatment.

The diet should be strictly aseptic (Diet List No. 1), and should in all particulars be the same as that recommended for nervous headache. When the stomach is considerably dilated, lavage should be employed once or twice a week, until the tongue, which is always coated, becomes clean. The bowels should be thoroughly and regularly evacuated. Constipation should be combated by the measures elsewhere recommended in this work. Antiseptic charcoal tablets (49), 
as recommended for migraine, should also be used in this disease, two or three to be taken after each meal. In both migraine and nervous apoplexy, it is sometimes necessary to use some intestinal antiseptic habitually, on account of the inability of the gastric juice to thoroughly disinfect and preserve the contents of the stomach during digestion.

For the immediate relief of an attack of nervous apoplexy, employ the following measures: Put the patient to bed in a quiet room, excluding the light and all sources of excitement. Apply fomentations $(\mathbf{1 0}, \mathbf{1 1})$ orer the stomach and abdomen; administer an enema (22) if the bowels are constipated; apply a wet girdle (9), and make hot or cold applications to the head when indicated. If the head is cold and the face pale, hot applications should be made; if the head is hot, hot or alternate hot and cold sponging of the spine (18), with cold applications to the head.

Disturbance of the Circulation.- Various disturbances of the circulation are common in dyspepsia. Coldness of the hands and feet, or the opposite condition, especially at night, accompanied by an aggravated burning sensation; unnatural heat in the liead, often accompanied by fulness of the veins of the forehead and neck, showing intense congestion; palpitation of the heart, especially occasioned by excitement of any kind, and sometimes coming on suddenly without apparent canse; intermittent action of the heart, with sudden sensations as of stopping, causing the patient to apprehend impending death; throbbing of the arteries in various parts of the body, this being. especially noticeable in irritable conditions of the stom- 
ach, which are often accompanied by strong pulsation of the aorta, felt at the pit of the stomach or lower in the abdomen; sudden flushing of the face, with or without any slight mental excitement, - these are the most common symptoms of this condition, some of which often occasion no little uneasiness on the part of the patient by exciting fears of organic disease of the heart, aneurism, or some other serious malady, which he thinks may end his life at any moment.

Treatment._- "General Tonic Treatment" ( see page 333 ), beginning with very gentle measures and progressing gradually, is one of the most effective means for relief of disturbances of the circulation. For temporary relief, hot or hot and cold applications may be made to the feet and legs, and prolonged cold applications to the parts which are excessively hot. Cold should be applied over the heart in cases of palpitation, and such measures employed as have been else. where recommended for relief of reflex symptoms due to an irritable condition of the abdominal sympathetic, as indicated by uneasiness at the stomach and pain in the region of the umbilicus (see page 193).

Nocturnal Asthma of Indigestion.- In rare cases the disturbance of the abdominal sympathetic is so great, on account of the formation of poisons in the stomach and bowels, and the failure of the liver to destroy them or the kidneys to eliminate them, that the patient becomes the subject of dyspnea, or difficulty in breathing, of the most distressing character. The attacks usually come on about midnight, - often before, but rarely later. The patient wakes suddenly from sleep to find himself sitting upright in bed, 
gasping for breath, and expecting every moment to breathe his last. These paroxysms are often ex. tremely distressing, and not easy to relieve except by the use of the stomach-tube, which usually quickly cuts short the attack. A large hot enema is often of value in these cases. The reason for the occurrence of this attack in the night is probably the lessened activity of the kidneys during sleep. During the daytime the poisons are eliminated with sufficient rapidity to prevent serious injury ; but the action of the kidneys being diminished during sleep, the poisons accumulate in such quantities as to produce the symptoms referred to. Those thus afflicted often experience relief after expelling a quantity of gas from the stomach. Eating suppers is also a cause of these attacks. The delay in the digestive process occasioned by sleep gives rise to fermentations and decompositions, to which the poisons above referred to are due.

Treatment. - An aseptic dietary (Diet List No. 1), from which flesh food of every description, including fish, oysters, etc., is strictly excluded, is indispensable to recovery in these cases. Cheese must also be prohibited. The most prompt relief is occasioned by a diet of kumyss, kumyzoon, or buttermilk (Diet List No. 25). In many instances a milk diet is sufficient, but in cases of dilatation of the stomach, milk is not suitable, hence buttermilk or kumyzoon is to be preferred. From two to three quarts of buttermilk should be taken daily. No other food but buttermilk or kumyzoon should be taken in serere cases, at least for a few days after an attack. After three or four days, granose (Diet List No. 25) and cereal foods, 
with fruits, may be used, but vegetables must still be avoided. A large hot enema should be taken daily, and the patient should take as much exercise out-ofdoors as possible. A short sweating bath will aid in the elimination of the poisons, but the hot bath should be followed by a cool shower, wet-sheet rubbing, or sponge bath, as a skin or heart tonic. A hot and cold full bath $(\mathbf{2 1})$ is a most excellent measure for these cases. Antiseptic charcoal tablets (49) should be habitually used in these cases, three or four being taken after each meal. It is probable that nervous or essential asthma is always due to poisoning of the nerve centers by substances generated by germs in the alimentary canal, and absorbed into the circulation.

"Stomach Cough."-This popular term has really more significance than many physicians are accustomed to allow. It is a very common observation that the stomach and lungs sympathetically affect each other. Most cases of consumption and chronic bronchitis are accompanied by stomach disorder; and, on the other hand, there are many cases in which dyspeptic conditions are accompanied by a troublesome cough, usually of a dry, hacking character, without expectoration. In not a few instances of this kind the cough is due to an elongated palate, or to congestion of the pharynx. Most of the remarkable cures of consumption by drugs are cases of this nature.

It must not be supposed, however, that every hacking cough is due to disease of the stomach. Many real consumptives make the mistake of thinking that their cough is wholly due to the stomach, and by procrastination lose their only chance for recovery. A cough 
accompanied by copious expectoration, or by a rapid pulse and night sweats, is rarely a "stomach cough." Serious disturbance of digestion is present, however, in nearly all cases of consumption. In fact, as a rule, failure of digestion precedes consumption in nearly all cases; hence in most cases of cough, except those in which the symptom is due to an acute cold, special attention must be given to the condition of the stomach.

Difficulty of breathing and a sense of suffocation are among the symptoms of dyspepsia in which the lungs are involved. The difficulty may arise from pressure against the diaphragm by a distended stomach, or through nervons influence.

Treutment. - In these cases, the cough will be permanently relieved only when the digestive disorder is cured. Temporary relief may be obtained by the use of the Perfection Vaporizer $(\mathbf{5 9 , 6 0})$. "General Tonic Treatment" (see page 333 ) and such other measures as are indicated by the symptoms of indigestion which may be present, must be perseveringly employed. . In cases of chronic cough, the vaporizer should be used several times a day. Temporary relief may be occasioned by gargling hot water in the throat for five to ten minutes two or three times a day. This is also a good curative means. Another excellent measure is the throat pack, applied thus : First place on the throat a cotton bandage consisting of several thicknesses of cloth wrung dry from cold water, and of sufficient length to extend around the throat. Cover this with several folds of flannel cloth of sufficient width to extend an inch or two over each side of the moist bandage, wrapping as tightly as possible without interfer- 
ing with breathing. This application should be made at bedtime. On rising in the morning, remove the bandage, bathe the throat and chest with cold water, and apply a dry flannel bandage to be worn during the day. The persistent use of this measure is one of the most effective means of relieving chronic irritation of the throat. In many cases a diseased condition of the tonsils or of the posterior portion of the nasal cavity is present, and requires proper treatment by a specialist.

Nervous Symptoms.-Most of the symptoms thus far mentioned have related directly to the digestive organs; but still other symptoms of importance remain to be noticed, which may be termed "nervous symptoms," as they are indirectly occasioned by abnormal conditions of the stomach and bowels. These symptoms, although they really arise wholly from disturbances of digestion, are often supposed to be indications of distinct and serious diseases of the nervous system. There is no doubt that faulty digestion is one great cause of nervous disorders, owing to the fact mentioned, symptome at first temporary becoming chronic, and functional disturbances giving rise to organic diseases.

The nervous disorders which accompany dyspepsia are due to four canses : (1) To sympathetic disturbance of function, through the nervous connections of the stomach ; (2) to impaired nutrition of the nervous system, defective digestion occasioning a poor quality of blood ; (3) to the presence in the blood of the products of indigestion, imperfectly elaborated food, acetic and butyric acids, and other poisons resulting from the action of germs ; (4) to retention of the excretions, ow- 
ing to the inactive condition of the liver, skin, and bowels, resulting from impaired nutrition.

The injurious elements mentioned, coming in contact with delicate nerves already weakened by impaired nutrition, increase their irritability, and occasion disordered actions of almost every conceivable variety, from the slightest degree of mental disturbance, as shown in the confusion of thought observed by the dyspeptic student or the forgetfulness of engagements by the business man, to complete loss of mental control, even actual insanity; and from the slight nervousness familiarly known as "fidgets," to the most alarming convulsive action, as in epilepsy and hysteria.

Trectment. - The symptoms referred to in the above paragraph should receive prompt and efficient attention, as they may be the beginning of grave nervous or mental disorders. The treatment most generally required is that indicated for slow digestion (see page 245). The coloclyster (23) or a laxative enema (25) not infrequently dissipates a distressing headache almost instantly.

Nervousness. - Many dyspeptics snffer more or less with an indescribable uneasiness, sometimes termed "fidgets." The limbs are chiefly affected. The patient finds it impossible to sit still. The lower limbs, especially, are kept in almost constant motion. Such persons find the confinement of sitting in church almost unendurable. This difficulty is especially troublesome in the afternoon and at night. It is often accompanied by peculiar sensations in the limbs, especially when sitting or lying, as "crawling," "prickling," "numbness," etc., which are sometimes thought to indicate a 
tendency to paralysis, but are merely signs of a weakened circulation and badly nourished nerves.

An allied and very singular sensation is that of motion. The patient, if very nervous, - and this is especially the case with young women,- - will often complain of feeling as though being carried involuntarily to different parts of the room, as from one corner to another, to the ceiling, to the window, or even into another room. Patients sometimes complain, also, of feeling as though some portion of the body were larger than natural; as, when lying down, the sensation will be that a limb, or a hand, or the head is immensely large. The delusion vanishes, however, upon the patient's attempting to move the particular part affected.

Treatment.-Hot and cold sponging of the spine daily (18), fomentation (10) over the abdomen at night, followed by the moist abdominal bandage (9) to be worn during the night, the cool morning sponge bath $(\mathbf{1})$, and the abdominal supporter (45) are the best means of palliation in these cases; but a thorough course of treatment and training at a well-equipped and scientifically conducted sanitarium is essential for a cure in most cases. General nutritive (page 331) or tonic treatment (page 333 ) should be employed.

Disturbances of Sight, Hearing, etc.-Nothing is more common in this disease than disturbancez of vision and of the various other special senses. Dimness of vision, deafness, ringing in the ears, extreme sensitiveness to noise and also to light, occur in many cases. Not infrequently the patient sees imaginary forms of varions sorts. Sometimes the intolerance of light is so great that the patient wishes to remain in a 
dark room. This is more common in women than in men. Often this extreme degree of apparent sensitiveness is more imaginary than real. The appearance of black or bright spots of various shapes and sizes, especially noted when stooping over, is another common symptom. Unnatural thirst, perversions of taste, peculiar sensitiveness of various parts of the body, are also symptoms worthy of notice.

Treatment. - In extreme cases, the "Rest-Cure" (page 329), followed by progressive application of nutritive and tonic treatment (pages 331 and 333 ), is required in addition to the application of such palliative measures as may be indicated by the general and local symptoms presented.

Nervous Diseases.-Functional nervous disorders, as hysteria, epilepsy, and even temporary paralysis, often originate in dyspepsia. Dr. Chambers, an eminent English physician, asserts that nine cases out of ten of hysteria are due to indigestion. This statement I have found abundantly confirmed by my own observation. In the treatment of epilepsy, I have observed in many cases, tenderness at the pit of the stomach, and a foul tongue. In some instances, no doubt, the stomach disease is secondary; but many times, at least, I believe that the latter difficulty is the primary affection. In many cases the remedy given to cure the disease is of a character to defeat the end desired, by deranging the stomach. The long-continued use of bromides is certain to derange the digestive organs, as this drug lessens the activity of the glands of the stomach. When bromides are used, a good intestinal antiseptic should be constantly em- 
ployed, as a means of preventing the fermentation of the food substances through the action of microbes which are left to, develop in consequence of a deficient supply of hydrochloric acid in the gastric juice. Antiseptic charcoal tablets are most suitable for this purpose (49).

If the tongue is coated, the treatment recommended for septic indigestion (page 251) should be employed. Careful examination should be made in relation to the condition of the stomach as regards dilatation (page 215). If possible, a test meal should be given and the stomach fluid examined, as much light is by this means often thrown upon the nature of the disease presented (page 134).

Unusual Drowsiness.- This symptom occurs most oiten after eating, though it is sometimes almost continuous. An hour or two after eating, the patient feels an almost irresistible desire to sleep. The sense of weariness and lassitude is sometimes so great that it is with difficulty that the person so affected can be induced to make an attempt to exercise. If he does so, however, he feels much better than if he yields to his desire to sleep. Exercise dispels the exhausted feel, ing, which is not fatigue; while if the patient allows himself to sleep, he awakes unrefreshed, and feeling really worse than when he lay down. In these cases, drowsiness is due to the development of poisonous substances through the action of germs upon the food, and their absorption into the system.

Treatment. - This symptom, often very troublesome, is frequently due to overeating, and is promptly relieved by eating a smaller quantity of food. Rapid 
eating is another common cause. The hot and cold trunk pack is a valuable measure of treatment, also fomentations to the abdomen, the moist abdominal bandage at night, and plenty of outdoor exercise. The measures recommended for "Simple Dyspepsia," or slow digestion (page 245), should be employed. For temporary relief the patient should drink one or two glasses of hot water, and practice deep-breathing exercises (38).

Sleeplessness. - This condition, quite the opposite of the preceding, is equally common, and often exists in the same individual, the person being very sleepy soon after eating, but wakeful at night. The patient is not kept awake by pain, but by simple nerrousness, by a sense of weight at the stomach, by morbid anxiety or fears, by burning of the feet and hands, or some similar cause. If sleep comes, it is not sound. There are troubled dreams, and the person awakes in the morning unrested and unrefreshed. These patients usually feel best in the afternoon and early erening.

In most eases of chronic insomnia the patient will be found to present a very foul tongue. There is often dilatation of the stomach, and generally many other symptoms pointing to disturbed digestion as the real canse of the sleeplessness.

Treatment. - Lavage of the stomach, the employment of a strictly aseptic dietary (Diet List No. 1),it may be necessary to diseard even milk if the stomach is dilated,- the use of antiseptic charcoal tablets (49), fomentations to the abdomen at night, the wet girdle (9) worn during the night, and "General Tonic Treatment" 
(page 333 ) are the measures specially indicated. The sleeplessness is the result of the circulation in the blood of substances of an irritating or exciting character. An aseptic dietary prevents the development of these poisons, and their elimination may be aided by copious water-drinking, exercise (37) in the open air sufficient to cause perspiration, breathing exercises (38), a light sweating bath once or twice a week $(\mathbf{1 6}, \mathbf{1 7})$, fomentations over the stomach and liver (10), sun or electric-light baths (15), whereby the skin is stimulated, etc. The Natural Abdominal Supporter (45), in cases which require it, affords great relief.

This most annoying and exhausting symptomsleeplessness - also requires attention to the following suggestions :

1. Retire early, having taken, an hour or so before, sufficient muscular exercise to induce slight weariness.

2. Eat nothing within four hours of bedtime. Going to bed without supper is perhaps the best of all remedies for sleeplessness. If there is a faint feeling at the stomach, drink half a glass of hot water. If this does not suffice, a mellow, sweet or subacid apple may be taken an hour before retiring, unless fruit occasions pain or acidity, in which ease one or two tablets of bromose may be substituted.

3. If feverish, the skin being hot and dry, take a light hand bath with tepid water upon retiring.

4. If the feet and hands are cold, employ the means elsewhere suggested for this condition (page 191).

5. Sleep in a cool room, taking care to see that the bedding is well aired and dry, and the room well ventilated. 
6. When nerrousness causes loss of sleep, there are various methods of indncing slumber, one of the most efficient being slow, deep, and steady breathing. By this means the lungs are filled with blood, and the brain is thus relieved of the congestion which causes wakefulness.

7. When the head feels cold, the indication is that there is not enough blood in the brain, and the head should be protected by flannel coverings. If the head is hot, a cloth moistened in cool or cold water may be applied, the hair being first wet.

8. In case the head is hot and full of blood, sleep may often be induced by raising the head of the bed six inches or more above the level of the foot. If the face is pale and the head cold, the foot instead of the head of the bed may be raised.

9. For sound sleep, the sleeping-room should not be too warn, and the patient should not be too warmly covered in bed.

10. Aroid exciting conversation or reading shortly before retiring.

Mental Disorders.-Until of late years it was not known that dyspepsia could be recognized by the mental and neirous symptoms alone; and eren yet the fact is not as well understood as it should be. It is thoroughly established, however, at the present time, that this is the case, and also that when the mental and nervous symptoms are most prominent, those which point directly to the digestive organs are the fewest and most obscure. Hypochondria has long been associated with indigestion, though often attributed to the liver. Students, clergymen, and other mental 
workers who suffer from cerebral hyperemia, or chronic congestion of the brain, are nearly always victims of indigestion, which results in defective nutrition of the nervous system. Gloomy apprehensions, forebodings, peevishness, perversity of disposition, religious despair, confusion of thought, loss of memory, absent-mindedness, and many other forms of mental disturbance are justly attributable to this cause, and disappear upon its removal.

The failure on the part of many physicians to recognize this class of cases has consigned many men of ability and influence to insane asylums, with no hope of recovery, who might have been saved to the world, their families, and themselves by a judiciously directed course of treatment. Hundreds of nervous women who have had their spines blistered and burned and cauterized for some supposed obscure nervous trouble, enduring years of torture, all without benefit, might have been made well by a few months of intelligent treatment for impaired digestion. A large number of both classes of invalids have come under my care, some of whom had been inmates of insane asylums for years without recovering, while others had baffled the skill of eminent neurologists; and having seen a large number of these cases recover mental soundness under rational treatment and with regulation of the diet and regimen, $I$ am convinced that much more attention should be paid to this class of dyspeptic cases. It is gratifying to see that particular attention is given to this matter in some of the best-regulated insane asylums.

Dilatation of the Stomach. - This condition is one which has, until recently, been rarely recognized. 
Ten or twelve years ago Glenard, an eminent French physician, called attention to the frequency with which both dilatation and prolapse of the stomach occur. By careful observation and logieal deduction, Glenard showed that a large share of the most obstinate symptoms encountered in dyspeptics is dependent upon a disturbance of the functions of the stomach arising from dilatation or prolapse of the organ. The principal cause of prolapse is constriction of the waist by corsets, tight skirt-bands in women, and the wearing of belts in men. In conjunction with dilatation and prolapse of the stomach, one or both kidners are frequently found prolapsed, the colon fallen below its normal position, and not uncommonly folded upon itself, and the liver and spleen also displaced in a downward direction. The consequences of these displacements are far more serious than any other visceral displacements which can occur in the body. Dislocation of the womb or ovaries in women, or the dislocation of a joint in either sex, is a matter of small consequence compared with displacement of the stomach, the liver, or the colon. Space will not here permit a consideration of all the symptoms which may grow out of these displacements. Suffice it to say that most of the numerous symptoms previously described as observable in indigestion may be the direct outcome of displacement or dilatation of the stomach, or prolapse of the other organs of digestion.

In prolapse of the stomach and other abdominal organs, the fact is in many cases quite apparent from the external form of the body. The waist is usually flat or depressed, and the lower abdomen excessively 
prominent. In addition, there is usually a forward carriage of the hips, round shoulders, depressed chest, with undue prominence of the spine between the shoulders, and forward carriage of the head. This condition is a prevailing one in women who have worn the ordinary conventional dress. A very common symptom of dilatation of the stomach is a plashing sound heard in walking or when rocking in a rockingchair after swallowing a quantity of water or other fluid. Some illustrations of cases of dilatation and prolapse of the stomach and other organs associated with indigestion, are represented in Plate IV, page 133.

Treatment. - In the treatment of this condition the most important measures are :-

1. Sufficient loosening of the clothing to permit proper expansion of the body at the waist, thus allowing the prolapsed organs to return to their normal position. For suggestions respecting proper dress, which is very essential to the cure of indigestion in women, see page 123 .

2. The support of the lower abdomen by means of the Natural Abdominal Supporter (45), whereby the displaced viscera are lifted up into position. The action of the supporter is practically identical with that of the hands when used to lift up the abdomen,a practice to which many such sufferers resort as a means of relieving uncomfortable, sinking, dragging, and other distressing sensations experienced in the lower abdomen. The employment of artificial support, however, is not sufficient to accomplish all that needs to be done in these cases. The viscera must be replaced by massage applied by a skilled masseur or 
masseuse who has been taught the normal position of the viscera, and how to distinguish each organ by palpation, and restore it to its normal position. By the employment of massage, the abdominal muscles may also be strengthened, a dilated stomach emptied, and congestion relieved $(31,32,33)$.

3. The application of electricity, particularly by the sinusoidal curent, * to the abdominal muscles, whereby they may be exercised and strengthened. I have found the application of the sinusoidal electrical current to the interior of the stomach, an excellent means of treatment in cases of dilatation of that organ. The application is made by passing a conducting wire through a stomach-tube introduced into the stomach. In one case in which every other measure had failed, the patient was in this way rapidly and permanently benefited $(67,68)$.

4. Carefully graduated exercise, particularly manual (42) and mechanical Swedish morements (page 33t), which is an important means of developing the muscles of the trunk in cases of this class. Bicycle riding and boating are also to be recommended, if employed intelligently, and not indulged to excess (37).

5. An aseptic dietary (Diet List No. 1) is absolutely essential in these cases, and should be scrupulously adhered to for an indefinite period. For three or four weeks the patient should be confined strictly to a dry diet (Diet List No. 2), no fluid whatever being allowed at meals nor until five or six hours after eating.

* For a description of the Sinusoldal Current, discovered by the author In 1883, see "The Graphlc Study of Electrical Currents in Relatlon to Therapeutics, with Special Reference to the Sinusoldal Current." Modern Mediclne Publishing Co., Battle Creek, Mich. 
Indeed, little fluid should be taken into the stomach at any time in these cases. If the patient is thirsty, the thirst may be relieved by introducing water into the bowels by means of the enema.

A person suffering from dilatation of the stomach should avoid swallowing plum stones and cherry pits. The stomach of an adult German was found, at the autopsy, to contain nine hundred and twenty cherry pits and seventeen plum stones, which had been retained for at least eight or nine months.

The writer has succeeded in curing two cases of exceedingly bad prolapse of the stomach by a surgical operation. The abdomen was opened by an incision extending from the lower end of the sternum to the umbilicus. The stomach, the lower border of which lay several inches below the umbilicus on the right side, was pulled into position, the lower border lifted as high as possible, and sutured to the median line by means of four silkworm-gut sutures, which were left buried in the tissues. A floating kidney was also found in one case, and sutured in position. Both patients made good recovery. The tongue, which had for years been extremely foul, became clean in a short time, and other disturbing symptoms which had been present for years soon disappeared.

Another method which has been employed is the enfolding of a portion of the dilated stomach. It is said that the enfolded portion disappears in the course of time. Surgical measures are seldom required, however. I have succeeded in restoring the prolapsed stomach to position and greatly diminishing the size of a dilated stomach in a great number of cases, by purely 
non-surgical means. The wearing of the abdominal supporter (45) and the development of the abdominal muscles by special exercises $(33,37)$, with the use of electricity, are sufficient to accomplish all that is required in the majority of these cases. Persons having once had a dilated stomach, however, must not expect to be so thoroughly cured that they can ignore a careful regulation of the dietary with reference to their condition. 


\section{TREATMENT OF DYSPEPSIA.}

As dyspepsia is not usually a fatal disease, thousands of people allow themselves to suffer from its pains and inconveniences for years without making serious efforts to recover. If anything is done, it is most likely to be a trial of some quack nostrum advertised on the fence or heralded in the daily newspaper as a "sure cure" for indigestion, its merits certified to by a long list of fictitious or purchased testimonials. Of course every effort of this sort makes the disease worse in the end, even though there may be apparent temporary relief. The sufferer finally settles down to the melancholy conclusion that his malady is incurable, and thus lives along in a wretched way until consumption, that dread disease which often follows close on the heels of the hydraheaded malady we are considering, claims him as a victim, and ends his misery.

The importance of giving serious attention to the treatment of this disease is further proved by the fact that many organic affections whicl, when once well established, it is impossible to cure, have their origin in indigestion. This is undoubtedly true of tubercular degeneration of the lungs and of other degenerative changes in various parts of the body. The same may also be said of many nervous affections. This accounts, in part at least, for the almost constant 
association of impaired digestion with consumption and various organic affections of the liver, kidneys, and other organs. In most of these cases, the best, sometimes the only, hope of a cure lies in the treatment and cure of the digestive disorder ; and, without doubt, if this could be accomplished sufficiently early, many cases of hopeless organic disease of the lungs and other organs might be prevented altogether.

The dyspeptic, of all invalids, needs to enter upon the work of getting well with a determination to succeed, and with a resolution to do all in his power to accomplish that end. Though an intelligent physician can do much, the patient can do vastly more for himself than any one can do for him. Indeed, he alone can control many of the conditions essential for the happy termination of his sufferings. If the dyspeptic would recover, he must seek carefully for each of the causes of his disease, and remove them. It is of no use to hope for recovery without doing this. If the cause is in the manner of eating, let him take care to eat properly. If he has erred in eating too much, or in eating improper articles of food, let him make a thorough reform in this regard. If the difficulty has been in overwork, too much anxiety, too little time for digestion, or too sedentary habits, he must get away from his care, his business, his writing-desk, and seek health in outdoor exercise, with happy, cheerful associations. The careworn, burdened mother must have relief from the tedium of her routine life. A journey, a visit to a friend, or some other means of diversion must be adopted. Whatever the cause has been, it must be removed. No medicine known, no matter how 
potent nor how skilful its administration, can antidote the effects of the transgression of physical laws. Nature is inexorable. She demands obedience, and will not be put off with any subterfuge.

The dyspeptic must also remember that it is not only his stomach which is diseased, but that every cell and fiber of his body is more or less injured by the failure of the stomach to supply proper nourishment for rebuilding the tissues, or by poisoning from the products of fermentation and decay which have been absorbed from the dilated or prolapsed and sluggish stomach.

It must not be expected that recovery from the consequences of a disease of many years' standing can be effected in a few weeks. Getting well is a process of growth, as much as the raising of a crop of corn or the development of a tree. The diseased stomach must be grown out of its vicious ways and into a healthy condition, under the influence of correct habits of eating and appropriate conditions of regimen and treatment. Many symptoms which have been long established will persist for some time after proper treatment has been established ; but this fact must not discourage its continuance. The patient should weigh himself at the beginning of treatment, and thereafter at least once a week. Loss of flesh for the first few weeks must not be a cause of discouragement, however, as it is a frequent occurrence. A gain in flesh may, however, be regarded as one of the most promising symptoms in cases in which any considerable degree of emaciation has existed.

It must not be supposed that any one plan will accomplish the result desired in all cases. There are 
various forms of dyspepsia, each of which requires special management, though the general principles laid down apply to all classes of the disease.

Diet and Regimen.- In the treatment of this disease, proper diet and regimen are of first importance. - It is necessary, however, that they should be most carefully adapted to the wants of each individual case, as nothing is truer than the old adage that "what is one man's meat is another's poison," when referring to cases of dyspepsia. The common plan of recommending some special dietary to all dyspeptics indiscriminately is a most pernicious one. We hear much of the "grape cure," the " beef cure," the "fat cure," the "cod-liver-oil cure," the "milk cure," and sundry other special diet cures of dyspepsia, as well as the "vegetarian cure." Each of these diets may be of service to some special case, but all are totally unfitted for all cases alike.

It is not an easy matter to induce individuals suffering with dyspepsia to deny the demands of appetite. In many cases the appetite is perverted, and the will is so weakened by long-continued disease that the patient loses self-control, and thus himself constitutes the most difficult obstacle in the way of his recovery. If a cure is expected, the directions given must be followed implicitly. In no other way can a confirmed dyspeptic hope for recovery. All but one or two requirements may be carried out, yet failure in one particular be sufficient to make all other efforts useless.

Dietetic Rules.-Although there is no such thing as a universal diet for dyspeptics, there are certain articles which must be discarded by all persons who havo 
weak digestion, and certain dietetic rules which must be conformed to by all. To the most important of these attention is now called.

1. Eat slowly, masticating the food very thoroughly. The longer the food remains in the mouth, the less time it will spend in the stomach.

2. In general, dyspeptic stomachs manage dry food better than that containing much fluid.

3. Avoid drinking at meals; at most, take a few sips of warm drink at the close of the meal, if the food is very dry in character.

4. Be careful to avoid excess in eating. Eat no more than the wants of the system require. Sometimes less than is really needed must be taken when digestion is very weak. Strength depends not on what is eaten, but on what is digested.

5. Never take violent exercise of any sort, either mental or physical, just before or just after a meal.

6. It is not well to sleep immediately after eating, nor within four hours of a meal.

7. Never eat more than three times a day, and make the last meal very light. For most dyspeptics, as well as healthy adults, two meals are better than more. The length of time between meals should not be less than seven hours, unless a special dietary is followed; 8:00 A м. and 3:00 P. M. are suitable times for meals.

8. Never eat a morsel of food between meals.

9. Never eat when very tired, whether exhausted from mental or physical labor.

10. Never eat when the mind is worried or the temper ruffled, if possible to avoid doing so. 
11. Eat only food that is easy of digestion, avoiding complicated and indigestible dishes, and partaking of few kinds at a meal. In severe cases it is often necessary to confine the diet for a time to one or two articles of food which are the most easily digested and assimilated.

12. Most persons will do well to diseard the use of meats, and add to their bill of fare an increased proportion in quantity and variety of cereal foods. The foods mannfactured by the Battle Creek Sanitarium Health Food Company, are to be highly recommended. They have been prepared especially for the use of invalids with feeble digestion, and are just what they claim to be. The writer has prescribed them in many thousands of cases, and finds them indispensable in the treatment of the hundreds of invalids with disordered digestion who come under his care annually.

As an aid to the reader in the selection of a diet suitable to his ease, the following diet lists are presented, which it is hoped will be found helpful :-

\section{DIET TABLES.}

The following is a list of dietaries which are based upon observations made in connection with the exact methods of analysis of stomach fluids and the study of digestive disorders, to which reference has already been made. They have been tested in the treatment of many thousands of invalids at the Medical and Surgical Sanitarium at Battle Creek, Mich., and have proved highly satisfactory. The made dishes called for are cooked in accordance with recipes given in "Sci- 
ence in the Kitchen,"' by Mrs. E. E. Kellogg, A. M., published by the Modern Medicine Pub. Co., Battle Creek, Mich.

\section{DIET LIST NO. 1.-ASEPTIC DIETARY.}

Especially adapted to cases in which fermentation is present, also in cases of chronic biliousness, sick-headache, jaundice, Bright's disease, gastrie and intestinal. catarrh, simple dyspepsia, most cases of hyperpepsia, and cases of hypopepsia not of extreme degree.

\section{A. Sterilized Foods Prepared without Milk or Eggs.}

Peas purée.

Lentil purée.

Beans purée.

Nuts purée.

Granose.

Granola.

Graham mush.

Oatmeal mush.

Mixed mush.

Gluten mush.

Granola mush.

IIominy flakes.

Oatmeal blane-mange.

Gluten water gruel.

Graham grits gruel.

Corn-meal gruel.

Graham gruel.

Oatmeal gruel.

Split pea soup.

Lentll soup.

Green peas.

Lentil toast.

Granose brown bread.

Zwleback.
Macaronl with tomato sauce.

Fruit toast.

Vegetable broths.

Corn soup.

\section{GRAINS.}

Barley gruel.

Corn-meal.

Cracked wheat.

Boiled wheat.

Molded wheat.

Pearled wheat.

Rolled wheat.

Graham grits.

Bran jelly.

Grain jelly.

Jelled oatmeal.

Rolled oats.

Baked potato.

Corn pulp.

Crystal wheat.

Sticks.

Crisps.
Bromose.

Nut porridge.

Nut butter.

Nut meal.

Sterilized butter.
Rolled rye.

Rice.

Rice water.

Rice molded.

Browned rice.

Hulled corn.

Gluten.

Grains of gold.

Hominy.

Popped Corn.

Corn gofio.

Wheat gofio.

Pulp succotash.

Kornlet and tomato.

Kornlet.

Unfermented breads prepared without milk.

FRESH FRUITS.

Grapes.

Ralsins.

Apples.
Berrles. Cherries. Currants.
Peaches.

Oranges.

PIneapples.
Pears.

Figs.

Bananas.
Melons. Dates. 


\section{FRUITS COOKED WITHOUT SUGAR.}

Baked apples.

Baked pears.

Baked apple dessert.

Baked tomato.

Prune marmalade.
Prune dessert.

Steamed prunes.

Stewed prunes.

Stewed raisins.

Stewed tomato.
Apple jelly without sugar.

Cranberries and sweet, apples.

Apples stewed with raisins.

\section{FRUITS COOKED WITH GRAINS.}

Pearled wheat with raisins.

Pearled barley with raisius.

Cracked wheat with steamed apple.

Farina with fig sauce. Rice with fig sauce.
Rice with raisius. Rice with peaches. Grabam grits with ralsins or figs.

Graham apple mush. Granola fruit mush.

Farina with fresh fruit. Prune ple with

Poached eggs.
Raisin gruel.

Macaroni with tomato sauce.

Banana toast.

Tomato toast.

Prune toast. granola crust.

B. Foods which Encourage Asepsis of the Stomach and Intestines.

All the articles named in the preceding list of sterilized foors prepared without milk or eggs, with the addition of the following :- -

Kumyss.

Kumyzoon.

Medium boiled eggs.

Beaten whites of eggs.
Fioated eggs.

Poached eggs.

Curdled eggs.

Curared eisted
Soft boiled eggs.

Beaten whole eggs. Cottage cheese.

\section{Foods which do not Ferment in a Stomach of}

\section{Moderate Digestive Vigor.}

All the preparations of grains, fruits, milk, eggs, and easily digested regetables in Diet Lists Nos. 1 to 10, only raw milk excepted.

- With this list it is necessary for many patients who have long suffered from dilatation of the stomach, to employ some intestinal antiseptic, of which the best is charcoal, in the form of antiseptic charcoal tablets (49).

\section{DIET LIST NO. 2.-DRY DIETARY.}

A dry dietary is indicated in cases in which there is dilatation of the stomach, with slow absorption. In 
cases of this sort, liquid foods remain too long in the stomach.

The chief purpose of a dry dietary is to secure thorough mastication of the food; hence the articles in the following lists should be taken without water, milk, or other liquid. Granose is an ideal article of food for a dry diet, as it stimulates the flow of saliva to a greater extent than any other food with which we are acquainted. Granose, combined with nuts, nut meal, bromose, or the yolks of hard boiled eggs, may often be eaten with advantage almost exclusively for a few days at the beginning of a course of dry dietary.

\section{A. Dry Diet.}

This list includes all the unfermented breads (Diet List No. 13 ), and in addition,-

Yolks of hard bolled eggs. Bromose (dry).

Granose.

Steamed figs.

Raisins.
Popped corn.

Sterilized butter.
Nut butter.

Nut meal.

Roasted almonds.

\section{B. Modiffed Dry Diet.}

This list includes all the preceding list (A), and in addition the following:

Poaehed eggs.

Curdled eggs.

Floated eggs.

Beaten whites of eggs.
Beaten whole eggs.

Nuts.

Granose grits.

Browned rice.
Popped corn.

Mashed peas.

Mashed beans.

Stewed prunes.

\section{DIET LIST YO. 3.-NITROGENOUS DIETARY.}

The nitrogenous elements of food are also peptogens; that is, they stimulate the secretion of gastric juice, and hence foods of this class are especially adapted to cases of hypopepsia and apepsia, in which 
the production of gastric juice is always deficient. $\mathrm{Al}$ bumen and casein are the most important nitrogenous elements. Egga consist chiefly of albumen; milk contains casein in abundance; nuts are rich in albumen and vegetable casein; peas, beans, and lentils contain a very large proportion of vegetable casein. At the beginning of a course of treatment, in extreme cases of apepsia and hypopepsia, it is sometimes necessary to confine the diet to a single nitrogenous food, as kumyss or kumyzoon. Later, eggs may be added advantageously, then some farinaceous food, as granose, with nuts, and still later the various unfermented breads and soft grain preparations; but dry foods are to be preferred.

Beans purée.

Peas purée.

Nuts purée.

Miḷ (Diet List No. 5).

Gluten biscuit, 1,2 .

Gluten meal.

Eggs (Diet List No. 6). Lentils.

Beans.

Peas.

NUT PREPARATIONS.

Almond meal.

Lentils with nuts.

Peas with nuts.
Beans with nuts.
Yuts purée.
Bromose.
Nut porridge.

Nut butter.

Nut meal.

\section{DIET LIST No. 4.}

This list consists exclusively of farinaceous preparations of a character easy of digestion.

BREADS.

(Diet List No. 13.)

TOASTS.

Cream toast. Snowflake. Gravy toast. Dry toast with hot milk. SOUPS.

Cream rice soup. Oatmeal soup. Cream barley soup. Corn soup. 
GRAINS.

Gluten mush.

Oatmeal mush.

Graham mush.

Granola mush.

Mixed mush.

Corn-meal.

Barley gruel.

Graham grits gruel.

Gluten water gruel.

Oatmeal gruel.

Milk gruel.

Graham gruel.

Corn-meal gruel.

Oatmeal porridge.

Oatmeal blanc-mange.
Macaroni baked with granola.

Macaroni with cream sauce.

Jellied oatmeal.

Barley milk.

Mllik panada

Bran jelly.

Rice.

Rice molded.

Browned rice.

Rice water.

Grain jelly.

Cracked wheat.

Molded wheat.

Boiled wheat.
Rolled wheat.

Wheat gofio.

Graham grits.

Gluten.

Granola.

Rolled outs.

Rolled rye.

Pearled whed.

Hulled corn.

Popped corn.

Hominy.

Hominy flalies.

Corn gofio.

Grains of gold.

Crystal wheat.

\section{DIET LIST Y0. 5.- MILK.}

$\begin{array}{llll}\text { Hot milk. } & \text { Boiled milk. } & \text { Cream. } & \text { Kumyss. } \\ \text { Buttermilk. } & \text { Sterilized milk. } & \text { Cottage cheese. } & \text { Kumyzoon. }\end{array}$

DIET LIST NO. 6.

A

This list is composed entirely of preparations of eggs.

Medium boiled eggs.

Hard boiled yolks of

Beaten whole eggs.
Soft boiled eggs.

Poached eggs.

Floated eggs.
Curdled eggs.

Beaten whites of egros.

\section{B}

The articles in this list contain milk or some cereal preparation in addition to eggs.
Gluten custard.
Granose custard.
Gluten meal custard.
Rice custard.
Bread custard.
Farina custard.
Rice with egg.
Cracked wheat custard.
Cream toast with poached eggs. Poached eggs on

DIET LIS'T No. 7.

Foods which are easily disintegrated, and hence adapted to cases of dilatation of the stomach.

\section{UNFERMENTED BREADS.}




\begin{tabular}{lll} 
Gluten mush. & \multicolumn{1}{c}{ GRAINS. } & \\
Granola mush. & Granola. & Browned rice. \\
Granose. & Giuten. & Corn goflo. \\
& Rice. & Popped corn.
\end{tabular}

FRESH FRUITS.

Strawberries. Grapes. Oranges. Ripe sweet apples.

\section{FRUITS WITHOUT SUGAR.}

Baked apples. Stewed prunes. Prune dessert. Fruit juice.

\section{FRUITS COOKED WITH GRAINS.}

Graham peach mush. Grape mush.

Granola grape mush. Oatmeal fruit mush. Granola fruit mush. Granola peach mush. Graham apple mush. Biackberry mush. Raspberry granola mush.

Granoia apple mush. Lemon bariey gruel. Raspberry grits gruel. Raisin gruel.

Grape gruel.

Lemon gluten gruel. Lemon oatmeal gruel. Boiled wheat.

Peas purée.

Lentil purée. Split peas.

Medium boiled eggs.

Beaten whites of eggs. Hard boiled yolks of eggs. Floated eggs.

MISCELLANEOUS.

New Era Kumyss. Kumyss nog. Kumyzoon.
Macaroni with tomato sauce.

Rice with fig sauce.

Farina with fig sauce. Pearled barley.

Grape toast.

Prune toast.

Banana toast.

Tomato toast.

Apricot toast.

Berry toast.

Peach toast.

Farina fruit moid.

Red rice mold.

Pearied wheat with raisins.

Pearled barley with raisins.
Green peas.

Vegetabie broth.

Corn pulp.

\section{EGGS.}

Poached eggs.

Curdled eggs.

Caramel-cereal.
Graham grits with raisins, dates, or figs.

Rice with raisins.

Wheat with berries.

Farina with fresh fruit.

Rice and stewed appie dessert.

Cracked wheat with steamed appie.

Apple macaroni.

Stewed fruit puddi'g.

Wheat with peaches.

Rice with peaches.

Prune pie with granoia crust.

Grauose shortcake.

\section{VEGETABLES AND LEGUMES.}

Kornlet.

Asparagus.

Nuts with lentils.

Soft boiled eggs.

Beaten whole eggs.

Bromose.

Nut butter.

Nut meal.

DIET LIST NO. 8.-ANTI-FAT DIETARY. An anti-fat dietary need not necessarily exclude all starchy foods. The most important points to be observed are - 
1. Limitation of food to the smallest amount with which the strength can be maintained.

2. Restriction of the diet to one article, or at most to two or three.

3. Avoidance of fats.

4. Avoidance of liquid foods of all sorts. If kumyss or kumyzoon is used, it should be made the chief dietary.

\section{Kumyss.}

Kumyzoon.

Granose (eaten dry).

Hard rolls.

Water biscuit.

Medlum bolled eggs.

Beaten whites of eggs.

Poached eggs.

Curdled eggs.

Floated eggs.

Soft boiled eggs.

Beaten whole eggs.
Vegetable broth.
Gluten.
Gluten biscuit.
Gluten wafers.
Gluten custard.
Sour apples.
Oranges.
Strawberries.
Bananas.

Grapes.

Lemons.

Celery (cooked).

Asparagus.

Tomatoes.

String beans,

Green peas.

Greens.

Sticks.

Zwieback.

\section{DIET LIST YO. 9.-FAT AND BLOOD DIETARY.}

Those foods which abound in starch, sugar, dextrin, and easily digested fats, are the most conducive to fattening. At the head of the list stands bromose, which contains one fourth its weight of emulsified, or partially digested, nut fat, and nearly forty per cent, of digested starch. The following articles are fattening in character :-

Milk (Diet List No. 5). Gralns (Dlet List No. 4).
Fruits and Nuts (Diet List No. 10).

Diet List No. 6.
Diet List No. 7.

Diet List No. 13.

Bromose.

\section{DIET LIST NO. 10.-FRUITS AND NUTS.}

\section{A.-FRESH FRUITS.}

$\begin{array}{lllll}\text { Grapes. } & \text { Oranges. } & \text { Peaches. } & \text { Pears. } & \text { Berries. } \\ \text { Melons. } & \text { Plneapples. } & \text { Bananas. } & \text { Figs. } & \text { Dates. } \\ \text { Raisins. } & \text { Apples. } & \text { Cherrles. } & \text { Currants. } & \end{array}$


B.-FRUITS COOKED WITHOUT SUGAR.

Baked apples. stewed raisins. Apples stewed with raisins. stewed tomato. Baked pears.

Unfermented wine. stewed fruits. Camned Pruits.

Almoncis. Hickory nuts. Filberts.
Stewed prunes.

Apple jelly without sugar.

Prune dessert.

Prune marmalade.

C. - FRUITS WITH SUGAR.
Cranberries and sweet apples. Baked apple dessert. Baked tomato. Stewed prunes.
Lemon apple. Citron apple. Bauanas in fruit sirup.

Nut meal. Nut porridge. Nut butter.

\section{DIET LIST NO. 11.-LIQUID DIETARY.}

A liquid dietary is especially indicated in fevers; and in some cases of painful dyspepsia, especially cases of hyperpepsia accompanied by much irritation, as indicated by pain on pressure over the pit of the stomach and pain after eating. The purpose of a liquid diet is to present nutriment in a form which will tax the stomach as little as possible, and secure the early emptying of the stomach by the passage of its liquid contents into the small intestine.

\section{Gluten gruel.}

Malted gluten gruel.

Fruit and nuts gruel.

Nuts and gluten gruel.

Vegetable soup(pea, lentil, Hot milk.

bean, tomato, rice, bar-Bolled mllk.

ley, and corn).

Vegetable broth.
Fruit juices.

Barley water.

Nut porridge.

Cream.

Buttermilk.

Custard.
Kumyss.

Kumyss nog.

Kumyzoon.

Cottage cheese.

Curdled eggs.

Beat'n whites of eggs.

Beaten whole eggs.

Bromose.

\section{DIET LIST NO. 12.-FEVER DIET.}

In fever the digestive powers are feeble. With the exception of boiled rice, only liquid food should be 
taken. Gruels and similar farinaceous preparations are most suitable, and they should be boiled several hours.

A. To be used during the active period of the fever, while the temperature is high :-

\begin{tabular}{|c|c|c|}
\hline Barley water. & Granola gruel. & Prunes purée \\
\hline Barley gruel. & Gluten gruel. & Bromose. \\
\hline Strained oatmeal gruel. & Fruit and nut gruel. & Kumyss. \\
\hline Bolled rice. & Unfermented grape & Kumyzooll. \\
\hline & Fruit juices. & \\
\hline
\end{tabular}

B. During convalescence, in addition to the above, the following may be used :-

Milk porridge.

Gream toast.

Granola mush.

Granose.

Poached eggs.

Custard.
Whipped egr.

Kumyss nog.

Stewed fruit.

l3aked apples.

Strawberries.
Peaches.

Grapes.

Zwieback.

Unfermented breads.

Toasted whole-wheat wafers.

\section{DIET LIST NO. 13. - UNFERMENTED BREADS.}

Whole-wheat puffs.

Corn puifs.

Graham puffs.

Rolls.

Toasted rolls.

Fruit rolls.

Breakfast rolls.

Graham gems.

Rye gems.

Blueberry gems.

\section{Snow gems.}

Whole-wheat gems.

Gluten wafers.

Whole-wheat wafers.

Toasted wafers.

Dyspeptic wafers.

Crisps.

Graham crisps.

Graham elackers.

Toasted beaten biscuit. Hoe eake.
Beăten biseuit.

Fig sandwich.

Apple sandwich.

Graham flakes.

Gluten.

Granola.

Crusts.

Zwieback.

Sticks.

\section{DIET LIST NO. 14.-FOR A BOTTLE-FED INFANT UNDER SIX MONTHS OF AGE.}

Sterilized cow's milk properly diluted with boiled water, barley water, or oatmeal water. Under three months of age, use one third milk ; three to six months, one half milk; after six months, two thirds milk.

Barley Water.-Boil one tablespoonful of whole barley in an enameled sauce-pan or a double boiler for five minutes, and throw the water away; then add one and one-half pints of water, slowly simmer down to one pint, then strain. To be used for diluting 
milk, or as a substitute for it when cow's milk does not agree, and in cases of vomiting.

Oatmeal Water.-One tablespoonful of fine oatmeal, one pint of boiling water. Boil for one hour, keeping the quantity of water good; strain. To be used instead of plain water for diluting milk.

Barley Wuter and Cream.-Five parts of barley water made as directed above, to one part of sterilized cream.

Substitute for Mother's Milk:-

Milk............................ oz.

Cream ........................... oz.

Bicarbonate of sola.................. gr.

Milk sugar.......................

Water to make.................... oz.

Gradually increase the quantity of cream and milk as the child adrances in age.

\section{DIET IIST NO. 15.-FOR A TEETHING INFANT.}

As soon as a child begins to cut its teeth, it should begin to use them. In addition to Diet List No. 14, the child may be fed stewed fruits in small quantities, those being avoided which require much sugar for sweetening. Such fresh fruits as strawberries, grapes (excluding the seeds and skins), ripe peaches, and very ripe sweet or subacid apples may be given, also baked sweet apples and pears. Granose eaten dry, or granose and granola eaten with milk or fruit juice, are perhaps the best of all cereal foods for an infant. Zwieback softened with milk or cream, and gluten gruel (Sanitarium Infant Food) are also excellent.

\section{DIET LIST YO. 16.-FOR A CHILI) FROM ONE TO FIVE YEARS OF AGE.}

The most suitable foods are those in Diet Lists Nos. 1 and 2, which a child from two to five years of age may eat without injury, provided, of course, that 
the food is properly masticated. Vegetables should be avoided until the second teeth begin to make their appearance, with the exception of the following : Asparagus, green peas, and baked sweet potatoes. Children subject to colic or other forms of indigestion should avoid potatoes. Purées of lentils, peas, and beans, and bean porridge may be allowed.

The articles included in Diet Lists Nos. 22 and 23 should never enter into the dietary of children, being still more unwholesome for them than for adults.

\section{DIET LIST NO, 17.- EATING FOR STREYGTH.}

Those articles are most useful in developing nerv. ous and muscular energy which are the most easily digestible, while at the same time possessed of the highest nutritive value. The nutritive value of the following articles is, on an average, with the exception of eggs, about three times that of beefsteak, while they tax the digestive organs less: Peas, beans, lentils, whole-grain preparations, bromose, granose, granola, unfermented bread. The food preparations mentioned in Diet List No. 1 are especially to be commended for producing strength.

\section{DIET LIST YO. 18.- DIET FOR DIABETES.}

An exclusive meat diet was formerly recommended for diabetes, but it is now known that such a dietary does not give the most satisfactory results, and involves the danger of sudden death from an excessive accumulation of tissue poisons which the kidneys are unable to eliminate.

$\begin{array}{llll}\text { Gluten biscuit. } & \begin{array}{l}\text { Asparagus, } \\ \text { Spinach. }\end{array} & \text { Kumyss. } & \text { Nuts. } \\ \text { Lettuce. } & \text { Greens, } & \text { Buttermilk } & \text { Nut butter. } \\ \text { Celery. } & & & \text { Nut meal. }\end{array}$




\section{DIET LIST YO. 19.-FOR RHEUMATISM AND GOUT.}

It is especially important to avoid an excess of nitrogenous food; hence meats of all kinds must be avoided. In cases of dilatation of the stomach, milk inust also be aroided. In most cases the articles included in the following diet lists are to be preferred :-

Dry foods (Diet List No. :).

Fruits and nuts (Dlet List No. 10). Aseptlc dietary (Diet List No. 1) and Dlet List No. 6.

DIET LIST NO. 20.

For gastritıs, gastric catarrh, and ulceration of the stomach.

Liquid foods (Diet List

No. 11).

Nut purée.
Nut meal.

Nut porridge.

Gluten custard.
Curdled eggs.

Egg-nog.

límyzoon.

\section{DIET LIST YO. 21.-FOR CONSTIPATION.}

Unfermented graham breads.

Cracked wheat.

Grits.

Oatmeal mush.

\section{Apples.}

Peaches.

llost raw frults.

Stewed fruits.

Crystal wheat.
Bromose.

Granose.

Zwieback.

Gofio.

Pop-corn.

\section{DIET LIST Y0. 29.-ARTICLES DIFFICULT OF DIGESTION.}

ANIMAL FOODS.

Pork.

Veal.

Goose.

Llver.

Kidney.

Heart.

Sausage.

Hard bolled eggs.
Scrambled eggs.

Cheese.

Hasberl and stewed meats.

Salted and smoked meats.

Melted butter and all animal futs.
Mackerel and all oily fish. Salt fish.

Dried and smoked fish.

Sardines and other fish preserved in oil.

Lobster, crabs, ete.

Cooked oysters and clams. Frled meats of all sorts.

\section{VEGETABLE FOODS.}

Warm bread, espe- Griddle-cakes.

cially when eaten Fich bread and vegewith butter.

Muffins.

Buttered toast.

Ples, eakes, and all sorts of pastry. tables.

Onions.

It ushrooms.

Pickles.

Tea, coffee, cocoa, chocolate.
Mustard, pepper, spices, and other condlments. Sugar, preserves.

Raw grains or vegetables. Raw or imperfectly cooked grains and regetables.

Ice-cream. 


\section{FOODS NOT EASY OF DIGESTION.}

The following articles are much more digestible than those just named, but are nevertheless so difficult of digestion that persons with dilated stomachs or weak digestive powers are compelled to wholly abstain from their use to avoid inconvenience from indigestion :-

ANIMAL FOODS.

\begin{tabular}{|c|c|c|}
\hline $\begin{array}{l}\text { Animal soups of all sorts. } \\
\text { Beef. } \\
\text { Lamb. } \\
\text { Game. }\end{array}$ & $\begin{array}{l}\text { Trlpe. } \\
\text { Turkey. } \\
\text { Duck. } \\
\text { Pigeon. } \\
\text { VEGETABLE FOODS. }\end{array}$ & $\begin{array}{l}\text { Codfish. } \\
\text { Oysters, raw. } \\
\text { Butter. } \\
\text { Roast meats. }\end{array}$ \\
\hline $\begin{array}{l}\text { Potatoes. } \\
\text { Turnips. } \\
\text { Cabbage. } \\
\text { Tomatoes. } \\
\text { Peas (whole and dried). } \\
\text { Beans (whole and dried). } \\
\text { Raisins and most dried } \\
\text { fruits. }\end{array}$ & $\begin{array}{l}\text { Plums. } \\
\text { Cherries. } \\
\text { Pineapples. } \\
\text { Beets. } \\
\text { Carrots. } \\
\text { Spinach. } \\
\text { Parsnips. } \\
\text { Vegetable soups. }\end{array}$ & $\begin{array}{l}\text { Salads of all } \\
\quad \text { sorts. } \\
\text { Currants. } \\
\text { Gooseberries. } \\
\text { Raspberrles. } \\
\text { Blackberries. } \\
\text { Rhubarb. } \\
\text { Jellies. }\end{array}$ \\
\hline
\end{tabular}

\section{DIET LIST NO. 23.-FOODS WHICH PROMOTE FERMENTA- TION AND DECOMPOSITION IN THE STOMACH.}

These are of three classes : A. Foods which are fermenting or decomposing when taken into the stomach; B. Foods which readily undergo fermentation in the conditions present in the stomach when the digestive vigor is somewhat impaired; and C. Foods which ferment in a dilated stomach.

\section{A. Fermenting or Decomposing Foods.}

Cheese.

Sour milk.

Sauerkraut.
Wine.

Cider.

Beer.
Wiid game and meat which has a gamey flavor.

B. Foods which Readily Undergo Fermentation.

Sugar.

Honey.

Candy.
Sirups.

Preserves and other sweets.
Meat.

Fish.

Oysters. 


\section{Foods which Ferment in a Dilated Stomach.}

Mushes. Gruels.
Soups. Potatoes and other vegetables.
Other soft or liquid farinaceous foods.

\section{DIET LIST NO. 24. - NCTRITIVE ENEMATA.}

1. Bromose and Egg.-Dissolve two ounces of bromose (Diet List No. 25) in two ounces of water. Mix with a beaten egg and a half teaspoonful of salt, and add water sufficient to make eight or ten ounces. Mix well, and administer with a suitable syringe at one feeding. Repeat five times in twenty-four hours, at intervals of four hours. This is the best material for rectal alimentation with which we are acquainted. Bromose may be obtained from the Modern Medicine Co., Battle Creek, Mich.

2. Egg Enema.-To two well-beaten eggs add half a teaspoonful of common salt, and water enough to make twelve ounces after thorourh mixing. This quantity is sufficient for one feeding. Use five times a day, at intervals of four hours. This preparation is less valuable than the preceding, but may be used in an emergency.

\section{DIET LIST NO. 2..-HEALTH FOODS.}

The following foods are prepared by the Battle Creek Sanitarium Health Food Company, Battle Creek, Mich., U. S. A. They are of great service in the treatment of many disorder's of the stomach :

Zuieback. - Ordinary fermented bread cut into. thin slices and baked a second time until lightly browned throughout.

Granose. - A preparation of wheat in which the grain is first thoroughly cooked, then compressed into extremely thin flakes, which are afterward baked until slightly brown. This food can be eaten and digested by many persons who can digest nothing else.

Granola. - A preparation of wheat, corn, and oats, mingled in such proportions as to render the mixture a perfect food, and treated in such a manner as to partly 
digest it. This food is capable of sustaining life any length of time.

Gluten Biscuit. - A small biscuit made of whent flour from which the starch has been carefully separated by washing. From this biscuit, carefully dried and browned, gluten meal is produced.

Caramel-Cereal.-A substitute for coffee, having a pleasant, aromatic flavor, and free from the unwholesome properties of tea and coffee.

Bromose. - This is a preparation having for its basis carefully selected and prepared nuts. It contains about forty per cent. of digested starch, twenty-four per cent. of digested fats, and twenty per cent. of finely divided albuminoids. It is one of the most valuable of all the fat- and blood-making foods.

Nut Meal and Nut Butter. - These are nut products of a very palatable, wholesome, and highly digestible character, and valuable substitutes for animal fats.

Gofio.- This is a preparation of parched grain much employed by the natives of the Canary Islands. After being parched, the grain is ground, and mixed with milk or water.

Tumyzom. - This is a lactated preparation of milk prepared by first sterilizing the milk, and then subjecting it to the action of a special lactic acid by which is produced a ferment whereby the sugar of milk contained in it is converted into lactic acid. It is a very easily digestible and highly nourishing article of food, and is of great service in certain forms of indigestion, as well as in diabetes and general malnutrition. Ordinary kumysis and buttermilk, though somewhat inferior, are useful substitutes, 
When kumyzoon is made the sole article of diet, it shonld be taken in quantities of two to three quarts daily and at intervals of four or five hours during the day.

Flesh Food. - It will be noticed that meats of all kinds are omitted from these lists. The reason for this is that flesh foods require strong digestive powers to disinfect and digest them, since they are not naturally adapted to the human stomach; hence, while they are apparently well tolerated by persons in health, a person whose stomach is in a diseased condition will do better to adhere as closely as possible to the natural diet originally intended by the Creator for the sustenance of human beings, as indicated by the command to Adam : "I have given you every herb bearing seed, which is upon the face of all the earth, and every tree, in the which is the fruit of a tree yielding seed; to you it shall be for meat." According to the nost eminent comparative anatomists, the anatomical structure and digestive functions of man agree entirely with those of such purely frugivorous animals as the chimpanzee, the orang-outang, and the gorilla.

Antisepsis of the Mouth.- - So long as the mouth is swarming with microbes, which is always the ease when the tongue is coated, and the teeth uneleanly and presenting unfilled cavities, thousands of germs are carried down into the stomach with every mouthful of food or drink swallowed. The first step toward asepsis of the stomach, and a most essential thing in the treatment of indigestion, is mouth cleanliness.

Modern researches have shown that nearly all diseases of the mouth, as well as a large share of the dis- 
eases of the stomach, are due to the action of germs which find lodgment there. The mouth is peculiarly exposed to the attacks of germs, as it is located at the very entrance of the body, and a portion, at least, of the respired air passes through it, and the germs readily find lodgment about the tongue, cheeks, between the teeth, and elsewhere. The mucus secreted by the glands of the mucous membrane lining the mouth, is, to some degree, antiseptic in eharacter, and possesses germicidal, or germ-destroying, properties to some extent. When the mouth is kept clean, this disinfecting mucus is capable of thoroughly protecting the structures of this portion of the body against the attacks of microbes; but when particles of food are left to lodge between the teeth, the germs, finding abundant soil in which to grow and multiply, become so numerous that the poisonous substances which they produce neutralize the antiseptic mucus so that it becomes powerless for protection.

Meat, more than all other foods, is injurious in this respect, for the reason that its fibers readily lodge between the teeth, and are not easily removed, and for the further reason that it furnishes a kind of soil in which germs grow with the greatest rapidity and develop the most virulent properties.

It is thus apparent that thorough cleanliness of the teeth and mouth is one of the most important hygienic measures. This fact becomes still more apparent when we remember that the act of eating or drinking and the frequently repeated act of swallowing to clear the throat from mucus, a practice which cannot be too much deprecated, are the means of carrying down into the stom- 
ach any microbes which may be present in the mouth. There are certain microbes, also, which seem to have their habitat in the mouth, particularly those of diphtheria, pneumonia, and consumption. It is not known that these germs propagate outside the human body, except under artificial conditions; but they find ready lodgment in the mouth, and are often present there in persons apparently enjoying perfeet health, waiting the opportunity when a severe cold or some other depressing agent shall, by reducing the resistance of the body, enable them to obtain a stronger foothold, and to manifest their presence by the characteristic symptoms of diphtheria, pneumonia, or some form of tubercular disease.

A very excellent means of cleansing the teeth is the Antiseptic Dentifrice prepared by the Modern Medicine Company, Battle Creek, Mich. The basis of this preparation is the extract of the bark of the famous soaptree of South America, one of the most remarkable cleansing agents known. The antiseptic properties of the dentifrice are due to the pure cinnamon oil which is added. These substances are combined with very fine precipitated chalk, making a detergent paste which is most effective in its cleansing action. Directions for the use of the dentifrice accompany each package. Simply rinsing the mouth with cinnamon water, prepared by arlding half a teaspoonful of cinnamon essence to half a glassful of water, is a useful means of disinfecting the mouth, to be used after thorough cleansing with a tooth-brush.

Artificial teeth must receive as scrupulous care as the natural teeth. It is considered best that they should 
be removed at night. After thorough cleansing they should be placed in a proper receptacle containing a quantity of pure water. This will prevent deposit from insects and the development of fungi, which often grow with great rapidity, especially in warm seasons of the year.

The distinguishing features of the several forms of dyspepsia and the general line of regimen and treatment necessary to effect a cure, are pointed out with greater definiteness than heretofore in this work in the following classification, which is based upon symptoms rather than upon the examination of the stomach fluid, which is the more scientific method, and the one habitually employed in the treatment of patients at the Battle Creek Sanitarium (see page 134). The suggestions made, if thoroughly understood and efficiently applied, cannot fail to be of great service, although based only upon symptoms. Those who can do so should by all means avail themselves of the advantages of treatment at a sanitarium where treatment is based on the examination of the stomach fluid.

Simple Dyspepsia.- This, the simplest and most common form of the malady, is sometimes called slow digestion. It is common in both men and women, and especially affects sedentary people and those nervous persons who eat rapidly and swallow their food without proper mastication. It is also common in persons whose teeth are defective. Its immediate cause is deficient activity of the muscular walls of the stomach and intestines. The symptoms are much the same as those which follow the taking of an excess of food, but are felt even when only a moderate amount 
has been taken. An hour or two after eating, a sensation of weight and oppression is felt. The discomfort continues for some hours, gradually. wearing off before the next meal. The appetite is usually fairly good, but often is not ready for the reception of food at mealtime, as the work of digesting the previous meal has not yet been completed. Sometimes there is considerable flatulence of the stomach, the eructations being tasteless, and never offensive; pain between the shoulders or beneath one shoulder-blade, and not infrequently in the region of the heart. Palpitation of the heart often occurs in the night, eausing great alarm on the part of the patient and his friends, who entertain fears of sudden death. Sleep is disturbed and unrefreshing. The tongue is frequently foul in the morning, with a bad taste in the month. All the symptoms mentioned are greatly exaggerated by a late supper, or by any unusual excess in quantity or quality of food. The bowels are apt to be constipated. When the difficulty has been of long standing, there is usually observed a marked disposition to sleep after meals, or unnatural sleepiness at other times, and a decided loss of vivacity and energy.

Examination of the stomach fluid in these cases shows a normal amount of work done, but the work is poor in quality. There is usually more or less decomposition of the food elements and the formation of abnormal products which, when absorbed into the blood, give rise to the general symptoms already referred to.

Treatment. - The indications in slow digestion are for improvement of the vigor of the body in general, 
especially of the organs concerned in the digestive process, and suppression, so far as possible, of the action of germs in the stomach and bowels. The following measures faithfully followed will be found serviceable :-

1. Eat simple, dry food, taking pains to masticate it very thoroughly. Granose, zwieback, rolls, and other unfermented breads constitute the staple articles of diet. To these may be added cooked fruits. The only raw fruits which may be eaten, in most cases, are perfectly fresh and well-ripened peaches, grapes, and strawberries. Very ripe sweet apples are well tolerated by most patients, if eaten with dry food and carefully masticated. Nuts, well masticated, are not objectionable. Milk is tolerated in some cases, but is better avoided by most patients, unless taken in the form of kumyss, kumyzoon, buttermilk, or cottage cheese. Sweets of all kinds must be carefully avoided, also drinking at meals, iced foods, rieh pies, pastry, rich gravies, pickles, condiments, and all foods difficult of digestion.

2. The cool morning sponge bath $(\mathbf{1})$, a warm bath once or twice a week $(\mathbf{2}, \mathbf{1 6}, \mathbf{1 7})$, followed by a cool shower (4) or sponge bath, general applications of electricity $(66,68)$, when possible, dry rubbing with a flesh-brush or towel at night before retiring, a salt glow (3) two or three times a week, are the most serviceable measures which can be employed at home. A hot bag may be applied over the stomach for half an hour after each meal, to hasten the digestive process. Gentle exercise or kneading of the stomach (31) after eating also hastens the digestive process. The moist abdom- 
inal bandage $(9)$ at night encourages intestinal activity, promotes secretion, and relieves congestion of the viscera. A dry flannel bandage worn about the abdomen during the daytime is also helpful, especially in cold weather. Regularity of the bowels must be secured, by the enema, if necessary, and by other measures recommended for the relief of constipation. Electricity applied to the spine and over the stomach is also a stimulant to the digestive processes, and useful to relieve constipation. The hot and cold full bath (21) is an excellent means of arousing activity of the secreting organs connected with digestion.

Swedish movements $(39,42)$, both manual and mechanical, are very helpful.

Some ten yeals ago the writer devised a vibrating chair which has been found very helpful in this class of cases, also a kneading apparatus by which the stomach and bowels may be kneaded in a most efficient manner. This, together with numerous other machines for applying morements to the body which have been devised by the author, are used in the Swedish movement department of the Battle Creek Sanitarium. The cannon-ball (Fig. 2, Plate VIII) is also helpful in stimulating the activity of the intestines, particularly as a means of relieving constipation.

3. Abundance of outdoor exereise is indispensable in the treatment of this as well as all other forms of indigestion. It not only creates a demand for food, but increases the ability to digest it. Horseback riding, bicycle riding, and boating are all excellent means of exercise, but are perhaps not preferable to useful labor in the cultivation of the soil. 
A course of "General Tonic Treatment" (page 333) is required. In very feeble patients a course of nutritive treatment (page 331) should be given at first as a preparation for the more vigorous tonic measures.

In this, as well as in all other forms of indigestion in which germs play an active part, some intestinal antiseptic, one of the best of which is antiseptic charcoal tablets (49), is found to be almost indispensable. Two or three tablets should be taken after each meal, and continued for several months.

Acid Dyspepsia.- This form of indigestion is that in which the digestion is so slow that the food undergoes fermentation, forming acids which irritate the stomach, and give rise to the same symptoms, much exaggerated, which are mentioned as pertaining to simple dyspepsia, with several additional ones, the prineipal of which are heartburn, regurgitation of intensely sour liquid from the stomach, and acid eructations; a white tongue, frequently with transverse fissures, often flabby and indented at the edges; acid saliva, eausing decay of the teeth; irregular bowels, either constipated or unnaturally loose; grinding of the teeth at night; and a reddish sediment in the urine. There is quite likely also to be pain at the pit of the stomach, with soreness on pressure. The digestion being very slow, portions of fermenting food remain in the stomach from one meal to another, so that acidity becomes habitual. No one case presents all of these symptoms, but several are often present.

Patients suffering with this form of dyspepsia are usually very thin and bloodless. Oecasionally, however, a case of the opposite kind is met, in which 
there is an abundance of tissue, though of a loose, flabby texture. Women suffer from acidity more frequently than do men.

Sweet foods, such as pastry, sweetcakes, preserves, etc., and in serere eases even starchy foods, especially vegetables, and sometimes also fruits, aggravate the tendency to acidity.

Treatment. - In these cases, patients are frequently found subsisting almost wholly on a meat diet, for the reason that they find flesh food less likely to form acids than are cereals and vegetable foods. This practice, however, aggravates the disetse in the end, and never effects a cure. The measures suggested for the relief of simple dyspepsia are equally appropriate for this condition. In addition, it is necessary in some cases - in all cases, in fact, in which the tendency to acidity is very pronounced - to cleanse the -stomach at least two or three times a week, and sometimes daily, by means of the stomach-tube (46). The best time for washing the stomach is at night. The extreme slowness of the digestive work renders more than two meals a day impossible without injury. The interval between the two meals should be at least seven hours, unless the quantity of food taken is very small, in which case it may be as short as four or five hours, and the number of meals may be increased. The longer interval, however, is preferable. Milk, unfermented bread, sugar, mncooked fruits, very acid fruits, fruits and vegetables together, and coarse vegetables should be especially avoided, also all articles difficult of digestion, and likely to cause fermentation (Diet Lists Nos. 22, 23). Wheat charcoal in its ordinary 
form, or better in the form of antiseptic charcoal tablets (49), is almost indispensable in the treatment of this condition.

A course of "General Tonic Treatment" (page 333), preceded in feeble cases with nutritive treatment (page 331 ), is indicated in these cases.

\section{Bilious or Foul Dyspepsia, or Septic Indi-} gestion.- The term bilious is used to distinguish this form of indigestion, not because either the liver or the bile is the immediate canse, but because of the bilions vomiting and sallow appearance of the skin which usually accompanies this condition. This is what is generally known as "biliousness." Acute dyspepsia of the same nature is termed "a bilious attack." Women, tailors, shoemakers, and sedentary persons generally, are particularly subject to this form of the disease.

As in acid dyspepsia, this form of indigestion differs from simple dyspepsia chiefly in the exaggeration of the morbid conditions present in that form of the disease. Digestion being still slower than in acid dyspepsia, the characteristic symptoms occur more remotely from the time of eating. The usual time for the appearance of the most marked symptoms is in the morning before breakfast. Headache, great flatulence, a very foul tongue, a bitter taste in the mouth, with nausea and finally vomiting of undigested and partially decayed food, indicate the inactivity of the digestive organs. When vomiting is continued, bile is generally expelled, the duodenum being affected, and taking part in the expulsive action. Diarrhea often accompanies and in some cases takes the place of the romiting. 
Owing to this thorough clearing out of the stomach and bowels, these attacks usually occur at intervals of at least several days. They are often periodical, however, recurring sometimes as often as once or twice a week, and again not oftener than once in two to four weeks.

In addition to the more important symptoms mentioned may be noted headache, often of a "splitting" or throbbing character, fetid eructations, and. nsually severe pain in the eyes.

Farinaceous foods give much less trouble than meats, especially fat meats. Vegetables eaten with fat, pastry, milk, meat which has been kept too long, and sometimes eggs, especially those not perfectly fresh, with albuminous and fatty foods generally, increase tlie symptoms peculiar to bilious dyspepsia, and bring on the attacks. In some cases the disease seems to be due to inability to digest starchy foods. This is especially true as regards the starch of regetables. Sufferers from this difficulty often make themselves worse by the use of purgative medicines.

Treatment.-Employ the same measures recommended for simple dyspepsia and aeid dyspepsia, taking care to give even greater attention to intestinal asepsis. Use an aseptic diet (Diet List No. 1). It may be necessary to resort to an exelusive kumyzoon or buttermilk diet (Diet List No. 25) for a short time. A diet consisting of granose (Diet List No. 25), with fruit, with or without the addition of nut meal, is an admirable dietary to be followed for a week or two in bilious dyspepsia. Granola, and most other cerenl foods, if properly cooked, agree well with this class of cases. As 
severe constipation usually exists, special attention must be given to the relief of this condition (see page 279). Lavage (46) one to three times weekly is necessary in the majority of cases of bilious dyspepsia. Charcoal or antiseptic charcoal tablets $(47,48,49)$ should be taken after each meal. A diet of granose or zwieback, bromose (Diet List No. 25), and stewed fruit, will, in most cases, effect a radical change within a few days in cases of this kind.

Painful Dyspepsia. - The chief characteristic of this disease is tenderness at the pit of the stomach, usually just at the lower end of the sternum, but sometimes affecting other portions of the stomach and also the duodenum, the pain in the latter region being often mistaken for some affection of the liver. In occasional cases the sensibility extends to other parts of the small intestine.

The tenderness described is almost always accompanied by a very unpleasant sensation at the epigastrium or extending along under the sternum, described by the patient as a "tearing," "burning," "rasping," "gnawing" pain, which comes on soon after taking food. This pain may be due to a morbid sensibility of the mucous membrane, or to a congested state usually called chronic inflammation of the stomach, or chronic gastritis, accompanied by a catarrhal secretion resembling that from any other mucous membrane in a like condition. When the pain is due to morbid sensibility of the stomach, it usually ceases when digestion is completed. When due to congestion, it is continuous, and is sometimes relieved to a degree by taking a moderate amount of bland food when the stomach becomes 
empty. The pain is often accompanied by throbbing of the aorta, felt at the pit of the stomach or below. Sexual excesses are a very common cause of irritability of the stomach in both sexes.

Not infrequently the congestion to which this pain is sometimes due is eaused by compression of the abdominal organs, obstructing free circulation. Hence women who wear corsets are very liable to be affected by it, theugh they will rarely admit the cause, and still more rarely can be induced to remove it. As a pithy writer once said, women have a strong "won't," which they sometimes oppose to all arguments, no matter how irresistible may be the logic.

Trutment.- - In this condition it is often advantageous to place the patient upon a diet of kumyzoon for a few weeks, alding eggs and some simple farinaceous preparations, such as granola or gluten mush (Diet List No. 25). The general measures employed may be the same as those for simple and acid dyspepsia. A hot trunk pack ( 7 ) usually gives relief from pain in the region of the stomach, abdomen, and spine.

Frequent eating, which is so often resorted to as a means of relieving the discomfort experienced in this form of dyspepsia, is a most pernicious practise, and serves only to aggravate the disease in the end. If eating is followed by pain, it may be necessary to confine the diet to the very blandest articles of food, such as gluten mush or gruel, granola mush, buttermilk, and purées of fruit, peas, or other legumes. Diet List No. $\tau$ is especially suitable.

In some cases it is preferable for a time to give the patient a small quantity of food three or four times a 
day, than a larger amount twice a day, as the stomach is thus not so severely taxed as by two hearty meals. When food is administered more than twice daily, care should be taken to give only the most easily digestible food. The two principal meals should be taken as far apart as possible, and the intermediate meals should consist only of liquid foods. We have found the hours of 8 A. M. and 3 P. M. the most suitable for the principal meals, and $12 \mathrm{~m}$. and 7 P. M. for the minor meals. Not infrequently the appetite is unnatural, the exercise of much will-power being required to control it. In many cases the pain occasioned by taking food may be relieved by the administration of twenty to thirty grains of subnitrate of bismuth before each meal. The remedy is a harmless one, but colors the stools black, from the action of the sulphide of hydrogen of the feces upon the bismuth.

Such articles as cracked and crushed wheat, oatmeal, graham bread, and other foods containing the coarser parts of the grain, are likely to do harm in this form of dyspepsia, the woody outside parts of the grain acting as a mechanical irritant to the sensitive mucous membrane of the stomach. It is this fact which has given seeming occasion for an ignorant class of writers to declaim so loudly against the use of whole-wheat flour. The fact that the coarser parts of the grain can with advantage be removed for certain cases, is no evidence against its wholesomeness or its utility in most other cases of indigestion. Granose is a most suitable food.

In severe cases, it is often necessary to put the patient on an extremely simple diet. As a usual thing, nothing answers the indications so well as milk. It 
should be taken as fresh as possible, and about as warm as can be borne with comfort, unless there is considerable fever, when it may be taken in small quantities iced. In extreme cases, the irritability may be so great that the food will be rejected if received in any but small quantities. If necessary, so small an amount as one or two spoonfuls may be given once an hour at first, both the quantity and the intervals being gradually increased until the necessary amount is taken at the usual intervals of meals. Then a little wellboiled and strained oatmeal or graham gruel may be added, the quantity being increased until the patient can bear semi-solid food. Many lives have been saved by this plan when death seemed imminent because of the inability to digest sufficient nourishment. Sometimes even milk is found intolerable; in such cases the most successful results are often obtained by the use of the white of egg beaten to a froth, and made palatable by the addition of a few drops of lemon or other fruit juice.

In this form of dyspepsia, the patient is apt to feel a terrible faintness as soon as the stomach becomes empty. This is in some degree relieved by taking food, which often leads the patient to resort to frequent eating when there is no requirement for so doing, and the result is only detrimental. The methods of relieving many painful symptoms which occur in connection with this disease have already been described in this work (see page 155).

In feeble patients, nutritive treatment, and in more vigorous cases nutritive treatment followed by tonic treatment (pages 331 and 333), should be employed, The application of galvanism $(\mathbf{6 9})$ to the spine and abdo- 
men is an excellent means of relieving the irritation of the abdominal sympathetic nerve, to which most of the symptoms are directly or indirectly traceable. The treatment indicated for hyperpepsia is necessary in many cases of painful dyspepsia.

\section{Gastric Neurasthenia, or Nervous Dys.} pepsia.- The sympathy between the stomach and the brain is very marked. Disease of the stomach may be produced by mental disorders, and on the other hand, various mental and nervous affections may arise from disease of the stomach. Indeed, cases sometimes occur in which the most prominent symptoms of dyspepsia, by which alone the disease may be recognized, manifest themselves through the nervous system. All the symptoms previously enumerated under the head of mental and nervous disturbances are observed in these cases.

The stomach symptoms of indigestion are sometimes so slight as not to be easily distinguished; yet there is undoubtedly a serious fault in the elaboration of the food. The process of digestion is left incomplete, and the blood becomes full of crude, unassimilated material, which not only does not impart to the tissues new life and vigor, but is a direct source of irritation and poisoning. The brain, being the most sensitive part of the nervous system, of course suffers most ; and hence there is abundant cause for the mental depression, unbalanced mental action, confusion of ideas, vacillation of judgment, perversity of disposition, and other kindred disturbances from which the nervous dyspeptic suffers.

Many persons, finding themselves in this wretched state, and not realizing the influence of physical condi- 
tions upon the mind, fall into hopeless despair, even when no intentionally wrong act has been committed. At first there is observed simply an exaggeration of real difticulties or misfortunes; but after a time the individual settles into a state of gloom, despondency, and mental depression, in which he suffers with troubles that are purely imaginary.

Nervous dyspepties often suffer much in mind from a morbid sensitiveness. They imagine themselves the subjects of criticism or ridicule, and therefore become morose, irritable, and exceedingly unhappy. Occasionally they find themselves haunted with evil thoughts, and sometimes with almost irresistible impulses to commit improper or eriminal acts, as blasphemy, suicide, ete. They are almost certain to imagine themselves suffering from many different diseases, usually of an incurable nature.

It is observed that mental disorders of the character described are often the result of intestinal dyspepsia, a form of the disease in which the local symptoms are less prominent than those which relate to the stomach, but equally grave.

This class of patients are often greatly injured by the use of mineral waters, which, not being absorbed readily, remain long in the stomach, and serve to inerease the nervous irritability.

In nervous dyspepsia the symptoms characteristic of the disease are due to an overexcited or irritated state of the sympathetic nerves. This is usually the result of dilatation or prolapse of the stomach, and is the direct consequence either of the strain upon the abdominal sympathetic nerves from the weight of the prolapsed 
stomach, or colon, or other abdominal organs, or of the absorption of the irritating products of the fermentation and decomposition taking place in the stomach, through its inability to unload its contents into the intestine with sufficient promptness.

Treatment. - In the treatment of gastric neurasthenia, or nervous dyspepsia, the services of a skilled physician are usually required. Many patients suffering from this disorder need a change of scene and surroundings, and relief from the home cares and business worries which have been largely responsible for the production of the disease. Hence they can be more successfully treated in a sanitarium than at home; and even in a well-equipped institution, the ingenuity and skill of the physician are often taxed to the utmost to bring about a successful recovery. Not the least difficult task to be accomplished is the deliverance of the patient from the morbid fancies which haunt him and make him in imagination a prey to almost every ill to which human flesh is herr.

The general measures of treatment are the same as those recommended for simple dyspepsia and acid digestion. A few special measures, however, will be found of service, particularly the hot and cold trunk pack (8), and the application of electricity to such remote parts as the arms, legs, back, etc. $(65,67)$. Headache, a symptom of nervous dyspepsia, is often relieved by the application of heat to the back of the head, a cold compress over the forehead, including also the eyes in cases in which the distress extends to these organs. In some cases an ice-bag to the 
back of the head, and a fomentation (10) to the top of the head or over the eyes, gives more immediate relief. Hot and cold sponging of the spine (18) is a valuable means for exciting the vasomotor centers of the spine. Galvanism of the spine and abdomen (69) promotes secretion, and relieves congestion of the viscera. As prolapse of the abdominal viscera exists in many cases of this class, the Natural Abdominal Supporter plays an important part in treatment (45). The same must be said of abdominal massage (29) and manual Swedish movements (40).

Hypopepsia and Apepsia.- It is not easy to determine the presence or absence of hypopepsia or apepsia without a careful examination of the stomach fluid; but when this condition is known to exist, - and it may be suspected in all cases of great debility, especially in those in which there is dilatation of the stomach and prolapse of the viscera in persons past middle age, - vigorous stimulating measures for the development of both muscular and secretory activity in the stomach must be employed. In these cases special attention must be given to the application of heat over the stomach, abdominal massage (29), hot and cold trunk pack (8), and manual Swedish morements $(39,41)$. Moderate exercise directly after eating encourages the secretion of gastric juice. Two other measures of treatment which have not been mentioned should be employed in these cases, especially when there is any considerable degree of tenderness over the region of the stomach :

1. The hot and cold douche over the stomach (20).

2. The hot and cold douche applied to the stomach 
itself through the stomach-tube, or hot and cold lavage (46). This may be employed whenever the stomach is washed out, if frequent lavage is necessary, or the stomach-tube may be introduced two or three times a week for the purpose of applying this vigorous stimulating measure, even if lavage is not necessary for the removal of fermenting food or mucus.

The dietetic measures recommended for simple dyspepsia should be employed in hypopepsia without acidity, and those for acid dyspepsia in hypopepsia accompanied by fermentation of the food.

What about Pepsin? - The use of pepsin in cases of so-called atonic dyspepsia, or hypopepsia, is very general, and many tons of this digestive ferment borrowed from the stomach of the hog are annually swallowed by human beings. It is doubtful, however, whether any benefit whatever is derived from the use of this substance. Some years ago Mosso, by experiments upon a dog, determined that the stomach of a $\operatorname{dog}$ of ordinary size was able to furnish pepsin enough to digest 70 kilograms, or 154 pounds, of albumin. This fact was ascertained by passing through the dog's stomach 2000 litres of water, acidulated with hydrochloric acid, to determine the degree of digestive activity.

More recently, Georges, according to Debove and Rémond, has demonstrated that the use of pepsin, papain, and pancreatin, either with or without hydrochloric acid, are valueless to supply a deficiency of pepsin formed by the glands in the stomach. My own observations exactly agree, in this respect, with those of the authorities mentioned. 
After having employed pepsin for many years, on the strength of authority, I long ago abandoned its use, together with that of other digestive ferments, with the exception of malt, having been utterly unable to see any good results from their use. What the stomach requires in cases of inactivity of the gastric glands, is not a substitute for the normal product of these glands, but an increase of the activity of the glands. Peptogens are needed rather than peptones, peptonoids, or pepsins. Proteid substances are the best of all peptogens. The natural flavors of foods and the dextrin developed by the action of the saliva upon starch are also peptogens of the highest value. The fact last mentioned furnishes another reason for the use of dry food, thus.securing thorough mastication.

One of the very best peptogens is gluten. Eggs and milk are also peptogenic substances of value, particularly eggs. The peptogenic power of gluten was well shown in a case examined in the Sanitarium Laboratory of Hygiene, in which, by adkling gluten to the test meal, the anount of free $\mathrm{HCl}$ was increased from .036 milligrams to .130 milligrams, and the total acidity from .260 to .354 .

The popular idea that pepper, mustard, and similar substances increase digestive vigol has been found by experiment to be erroneous. These substances, like common salt in excess, increase, the flow of mucus without increasing the production of pepsin or hydrochloric acid. The production of pepsin and hydroehloric acid can be increased only by natural stimuli, especially by peptogenic food substances. These consist (1) of proteid substances, which are intended to be digested 
by the action of pepsin and hydrochloric acid ; (2) of dextrin and maltose, the substances which naturally precede peptone in the order of production in the digestive process; and (3) of food-flarors, which by the reflex action produced increase the activity of all the digestive glands as a preparation for the digestive act to follow.

The only medicinal agents which I have found of any value whatever in the treatment of hypopepsia and apepsia, have been antiseptics, such as cereal charcoal or charcoal in the form of antiseptic charcoal tablets (49) previously described, lactic acid in twenty-drop doses, and hydrochloric acid in doses of fire to eight drops (51), well diluted, half an hour after eating. The use of antiseptics is a matter of great importance in these cases, in many of which the continued use of an antiseptic is necessary, owing to the failure of the stomach to secrete a proper amount of hydrochloric acil. The antiseptic charcoal tablets are a remedy which c:in be used for a long time without detriment. Hydrochloric acid may sometimes be used for a few weeks with advantage, but should not be depended upon for a great length of time. The stomach must be trained to make its own hydrochloric acid in proper quantity ; otherwise no permanent improvement will be secured. In hypopepsia and apepsia great care should be taken to avoid chilling the stomach by very cold foods.

General tonic measures are required (page 333 ). In very feeble patients, the rest-cure (page 329 ), may be first needed for a time, and it is sometimes wise to enploy nutritive treatment (page 331) for a few weeks before beginning more vigorous tonic measures. 
Hyperpepsia.- The symptoms presented in this condition are in many cases almost identical with those of ordinary acid dyspepsia. The condition itself, however, is very different, the acidity of the stomach fluid being due to an excessive secretion of hydrochloric acid rather than to fermentation. Yet in some cases there is both an excessive formation of hydrochloric acid and fermentation. This combination produces the most aggravated of all forms of acid dyspepsia. The acidity of hyperpepsia differs from that of acid dyspepsia in the fact that it usually makes its appearance soon after eating, often within an hour, and increases as long as the food remains in the stomach. Not infrequently the patient suffers from headache and other reflex symptoms during the entire period ocenpied by the digestive process, almost instant relief being experienced as soon as the stomach becomes empty. An examination of the stomach fluid by the method explained elsewhere in this work (page 13t) is necessary to distinguish with certainty between hyperpepsia and ordinary acid dyspepsia.

Trectment. - The treatment of hyperpepsia is radically different from that required for the relief of acid dyspepsia. It is necessary that the activity of the stomach glands should be diminished. This is best accomplished by the following means: Rest for one or two hours after eating; an aseptic or dry dietary (Diet Lists Nos. 1 and 2); careful avoidance of the use of meats, condiments, and of all articles difficult of digestion (Diet List No. 22). When fermentation is present, which is frequently the case, especially when hyperpepsia is accompanied by dilatation of the stomach, articles likely to ferment (Diet List No. 23) and preparations 
of milk, with the exception of -kumyss, kumyzoon, and buttermilk, must be carefully avoided. A hot or cold pack (8) before meals, a wet girdle (9) at night, general tonic treatment or, in case of feeble persons, tonic treatment preceded by nutritive treatment (pages 333 and 331) are the measures indicated. The patient should take abundance of outdoor exercise daily. Swedish movements $(39,40)$ may be employed with advantage, but movements should not be taken within two or three hours after eating.

In very severe cases it is sometimes necessary to administer soda in ten- to twenty-grain doses soon after eating, to neutralize, in part, the excessively acill gastric juice.

Catarrh of the Stomach.- This condition is characterized by the production of an excessive quantity of mucus in the stomach. The tongue is heavily coated ; there is loss of appetite, either partial or complete; usually tenderness at the pit of the stomach; constipation; in many cases dilatation or prolapse of the stomach; and not infrequently occasional attacks of jaundice, accompanied by severe pain similar to that of gall-stone, but unlike it, in that it does not terminate suddenly, as in the passage of gall-stones. Jaundice in these cases is always much more persistent than that which appears in connection with gall-stones.

Treatment.-In the treatment of catarrh of the stomach, the measures recommended for simple dyspepsia and painful dyspepsia should be employed. An aseptic diet (Diet List No. 1) agrees best with these cases. All articles difficult of digestion (Dict List No. 22) and liable to ferment in the stomach (Diet List No. 
23) should be carefully avoided. Granose, granola, and rarious cereal preparations, with fruits and nuts, are most suitable. Lavage (46) should be practised daily or every other day, if the tongue is very foul, and. inarked symptons of indigestion are present. "General Tonic Treatment" (page 333) should be given in connection with the other measures suggested. In many cases a considerable quantity of water will be required to wash out the mucus which has accumulated.

The moist abdominal bandage (9) and the hot and cold trunk pack (8) should be assiduously used, and for a long time. Astringents are of no value. If considerable irritation exists, and pain after eating, twenty or thirty grains of subnitrate of bismuth (62) may be taken before each meal.

Starch Indigestion.- Indigestion of starch may be the result of several causes:-

1. Insufficient cooking of the food.

2. Insufficient mastication, and consequent insufficient insalivation.

3. An excessively acid gastric juice.

4. Improper food combinations, as the eating of vinegar or some strong vegetable acid, like lemon juice, in connection with farinaceous foods.

5. The taking of a large quantity of fluid with the food, thus diluting the digestive juices to an excessive degree. The starch of vegetables is much more difficult of digestion than that of most grains. Potatoes seem to be more liable than other vegetables to give rise to indigestion in these cases, perhaps from the fact that they contain a larger quantity of potash than other vege- 
tables. The potash salts are very irritating to a sensitive stomach.

The symptoms of starch indigestion are flatulence, distention of the stomach, in some instances acidity, with eructations of gas and sour liquids - in other words, the symptoms of acid dyspepsia.

Treatment. - The treatment consists of the following measures :-

1. The remoral of each of the causes mentioned, which have been sufficiently clearly indicated to render further directions unnecessary.

2. The use of dry and thoroughly sterilized food. Grerms are the active cause of the indigestion of starch. Experiments made in the Laboratory of Hygiene of the Battle Creek Sanitarium have shown that a test meal consisting of granose furnishes no germs whatever, all the microbes swallowed with the food being destroyed by the normal gastric juice produced when a dry and well-sterilized food such as granose is eaten with thorough mastication.

3. The use of some good antiseptic, as antiseptic charcoal tablets.

4. The employment of food substances free from starch, as gluten, malted gluten, and in some cases malted milk, nuts, nut meal, eggs, kumyss or kumyzoon, buttermilk, and cottage cheese. A nitrogenous dietary (Diet List No. 3) or a diet of fruits and nuts (Diet List No. 10) may be advantageously employed. Marked benefit is frequently experienced from the use of a strictly dry diet (Diet List No. 2).

It is particularly important to avoid the use of pota- 
toes and starchy vegetables. Many persons can digest well-boiled rice when no other form of starchy food is tolerated. Rice starch seems to be more easily digested than other forms of starch. It is necessary, however, that the rice should be cooked in such a manner that the grain will remain whole and as dry as possible.

In most cases great bencfit may be derived from the use of bromose.

Intestinal Indigestion.- The most prominent symptoms of intestinal indigestion are distention of the bowels with gas, colic pains, tenderness about the umbilicus, fetid fecal discharges, loose, watery stools, and in some instances jaundice, arising from extension of intestinal catarrh into the bile ducts. Intestinal indigestion invariably occur's secondarily to gastric ind1gestion. Hasty mastication, whereby food enters the stomach imperfectly chewed, and after a time worries itself down into the intestine, there to become a source of irritation, is one of the most common causes of intestinal indigestion. The use of condiments, large quantities of sweets, meat, coarse vegetables, candies, ices, etc., are also common causes of intestinal dyspepsia.

Treatment. - Well-cooked cereals, cooked fruits of all sorts, kumyss, kumyzoon, or buttermilk, eggs, and nut meal should constitute the dietary in cases of intestinal dyspepsia. Drinking at meals should be carefully avoided. Antiseptic charcoal tablets (49) or their equivalent are of great service in aiding the stomach in destroying germs, to the action of which the disturbance in the intestines is chicfly due. It has been recently shown by carefin bacteriological study, that 
the intestines always contain fewer germs than the stomach; -so that if the contents of the stomach are made perfectly sterile, it is reasonable to believe that the number of germs in the small intestine may thus be rapidly diminished.

Fomentations to the abdomen at night (10), followed by the moist abdominal bandage worn over night (9), also a cool sponge bath (1) every morning, preceded, if necessary to aroid chilliness, by fomentations to the spine and vigorous rubbing with a coarse towel or a dry flosh-brush, are the most important measures of treatment which can well be employed at home. If there is prolapse of the bowels, the Natural Abdominal Supporter (45) should be worn. The bowels must be kept well evacuated, by means of the enema (22), or coloclyster (23) administered daily, if necessary. It should be employed quite hot in cases of looseness of the bowels and diarrhea, and at a lower temperature in cases of constipation. If much mucus is present after the bowels have been thoroughly washed out by the coloclyster, a quart of water containing one dram of tannin may be taken and retained for ten or fifteen minutes, the remainder then being passed off. See also measures recommended elsewhere for diarrhea.

Torpid Liver.-Errors in diet may justly be said to be the most frequent of all the causes of torpidity of the liver. Fashionable dinners, late suppers, overeating, and especially the excessive use of fats, sugar, pastry, condiments, alcoholic drinks, tea and coffee, are the most common causes of inactivity of this organ. The free use of mustard, ginger, pepper, curry powder, and other irritating condiments, leads to the 
almost universal prevalence of this disease in many tropical countries. In addition, sedentary habits, the use of tobacco and other narcotics, restriction of the liver by wearing tight clothing, and also malarial diseases, may be mentioned as frequent causes of torpidity of the liver. The prolonged use of laxative medicines, "after-dinner pills," and the various drugs recommended for constipation are most prolific sources of torpid liver. The same may be said of mercury, although this drug is less frequently used than formerly. The liver possesses the curious property of being able to retain in its structure metallic poisons which may be brought to it in the circulation, so that the injury received from a mercurial course is apt to be more or less permanent.

Trectiment. - In severe chronic cases of this affection the patient must carefully avoid the use of fats, sugar, condiments, and alcoholic drinks. Regulation of the diet is a positive necessity in the radical treatment of this disease. Tobacco, if used, must also be discontinued. If the patient's habits are sedentary, he must begin a course of regular, systematic exercise, and should strive in every way possible to build up his general health. Food should be taken in moderate quantities, and should consist chiefly of grains, nuts, and fruits. Some are obliged to avoid the use of milk, while with others it does not seem to disagree. In addition to these general measures, the patient, if not emaciated, may with advantage take two or three rapor baths (16) or packs (6) a week for two or three weeks. The wet girdle (9) should be worn night and day. The use of the hot and cold douche over the liver (20) is very efficient, 
Central galvanization may also be applied with advantage. The use of the various liver medicines which are recommended for this very common affection will do more harm than good. The best that any of these drugs can do is to whip up the flagging energies of the already overworked organ, without in any way lightening its burdens, or giving it increased strength to perform the labor required of it. The repeated use of remedies of this kind greatly aggravates the trouble, as it only increases the inactivity of the organ. Mercury does not stimulate the liver, as is commonly supposed, but, on the contrary, lessens its activity.

An aseptic dietary (Diet List No. 1) should be adopted. Kumyzoon or buttermilk should be used in place of milk, and in many cases nut meal (Diet List No. 25 ) should be substituted for milk and butter. Fomentations inay be applied over the liver daily with advantage, and a cold sponge bath taken every morning.

Vigorous outdoor exercise is a matter of very great importance. Breathing exercises (38) are especially helpful, as they aid the circulation of the blood through the organ, thus diminishing congestion. Socalled torpidity of the liver is chiefly due to the formation of poisonous substances in the stomach. The liver must be relieved, as far as possible, of all unnecessary burdens by the discarding of all substances which are readily decomposable (Diet List No. 23). Intestinal antisepties are of great value. Charcoal or charcoal tablets $(47,48,49)$ may be constantly used with advantage by persons who habitually suffer from biliousness or torpidity of the liver. 
Infantile Dyspepsia.- The most common symptom of infantile dyspepsia is vomiting. When the matters vomited are very sour, the child is suffering with acidity of the stomach, which may be the result of overeating or of the use of sugar or starchy food. Green, offensive bowel discharges indicate decomposition of the contents of the intestines in consequence of imperfect digestion. The green discharges are generally preceded by discharges in which lumps of curd are seen, indicating that digestion is imperfectly performed. After awhile, an irritation of the intestinal canal arises from the contact of hard, undigested curds which should have been digested in the stomach, and the discharges become more offensive in character, and are likely to contain considerable mucus from catarrh of the bowels. Clay-colored stools indicate an inactive condition of the liver, or an obstruction of the bile ducts, probably in consequence of the extension of the intestinal catarrh into the bile ducts. When the stools continue greenish, sour, or fetid, the child sometimes shows marked symptoms of wasting, becoming thin and wrinkled, - the countenance wearing an old look, - weak, peevish, -and restless. In many cases, the child has convulsions in consequence of its weakened state, and sometimes dies in one of them. In other cases, it dies from exhaustion. When vomiting is the principal symptom, the difficulty frequently increases until the little sufferer is unable to retain anything upon its stomach.

The chief causes of infantile dyspepsia are, overfeeding, the use of unclean bottles or of stale or unsterilized milk for bottle-fed babies, improper food, and 
errors in diet or ill health on the part of the mother during the nursing period.

Treatment. - The dietary should be adapted to the age of the child (Diet Lists Nos. 14, 15, 16). During the attack a special dietary will be required. If the child is six months or more old, milk should be withheld for a few days, thin, well-boiled barley or oatmeal gruel being substituted. The beaten white of eggs dissolved in water or a thin gruel is also an excellent diet in cases of this sort. General tonic measures are required (page 333), and the other measures recommended for relief of slow digestion (page 245).

An Important Caution.- It is of great importance to recollect that the special directions for the diet in different forms of dyspepsia here given are not intended as rules to be followed for any great length of time. In many cases it is necessary to adhere strictly to the special dietary only for a few days, when it may by degrees be made to include a larger variety of foods. This fact must, however, be impressed upon the mind of the dyspeptic, that when he finds himself well again, he must not make the error of supposing that the principle "once in grace, always in grace" in any sense or in the smallest degree applies to the improved state of his digestion. Although the stomach may be restored to a sufficient degree of health and vigor to enable it to do its duty well under favorable circumstances, it will be certain to relapse into a diseased state again as soon as those conditions are no longer supplied. The dyspeptic must make up his mind to study carefully the laws of good digestion, and apply them to his own case, 
not only as a means of recovering his health, but as an essential for keeping well when he has recovered. When the stomach has once lost its natural, healthy tone, it will never again bear the degree of abuse which it may have endured for a considerable time before breaking down.

In the treatment of dyspepsia, attention to the principles of hygiene and the application of hygienic remedies are of first importance. Indeed, it is by these agents that nature is aided in her restorative work more than by any others, and upon these the most skilful and successful of those who have given great attention to the treatment of the functional diseases of the stomach find it safest to rely. Undoubtedly there are cases which may be benefited, and the work of cure hastened, by the employment of medicinal agents ; nevertheless, the abuse of drugs is so very great, and has been the direct cause of so many cases of confirmed dyspepsia, that it would seem fir better to do without them altogether than to use them as they are frequently employed. An eminent writer on this subject, in referring to the treatment of dyspepsia, says, "My main object in the treatment is to prevent the sufferers from resorting to drugs, which in such cases not only produce their own morbid conditions, but also confirm those already existing." *

The extensive and often habitual use of alkalies for acidity, of purgatives for constipation, nervines and opiates for sleeplessness, and " after-dinner pills" to goad into action the lagging stomach, has been a potent factor in the production of a large class of most inveterate dyspepsias. This sort of treatment for dyspepsia 
cannot be too much discouraged. Especially to be discountenanced is the wholesale employment of "liver pills," "stomach tonics," "anti-bilious pills," "bitters," and the entire genus of quack nostrums and proprietary drugs.

Chronic Catarrh of the Stomach.- The symptoms of chronic gastric catarrh are pressure and fulness at the stomach after eating, flatulence, heartburn, little or no appetite, vomiting, water-brash, tenderness at the pit of the stomach, sliny tongue, bad taste in the mouth, obstinate constipation, occasional jaundice, mental depression, lassitude, pains in the face and limbs, and sleeplessness.

The chief causes of gastric catarrh are chronic indigestion, the use of flesh food, hasty eating, overeating, the use of condiments, alcoholic liquors, tea, and coffee-in fact, whatever tends to excite, irritate, or interfere with the normal functions of the stomach may produce chronic gastric catarrh. In this disease there is an abnormal development of microbes, which, being imbedded in the mucus which covers the lining membrane of the stomach, are retained and attack the food, giving rise to fermentation, and the consequent production of poisonous substances which increase the gastric irritation and catarrh, and when absorbed into the system, give rise to the great variety of nervous symptoms present in this disease.

Treatment.- Proper regulation of the diet is the most important measure. Coarse foods must be avoided when there is much local irritation, the diet being restricted to well-disintegrated foods. An aseptic diet, and in severe cases a liquid diet-exclud- 
ing, however, milk (unless in the form of kumyzoon, kumyss, or buttermilk), beef tea, and meat broths of every description - must be employed for a considerable length of time. Condiments must be avoided, as well as all exciting food substances, together with tea and coffee, and alcoholic liquors in every form. The treatment to be employed is the same as that recommended for "Bilious Dyspepsia" (page 251), a frequent precursor of this disease. The moist abdominal bandage (9) worn at night; the hot and cold trunk pack daily $(\boldsymbol{8})$; the daily cool morning sponge bath (1); lavage of the stomach (46) two or three times a week, if necessary to remove accumulated mucus or to suppress fermentation; the graduated enema (24) when constipation exists; and all measures for the improrement of the general health which have been mentioned elsewhere in this work, are the most reliable means of combating this disease.

"General Tonic Treatment" (page 333) should be employed, beginning with "Nutritive Treatment" in the case of feeble patients.

Ulcer of the Stomach.- The symptoms of ulcer of the stomach are pain in the stomach and in the spine opposite the stomach, increased by taking food, especially hot drinks and sugar; tenderness of the abdomen, particularly over the stomach; violent beating at the pit of the stomach ; vomiting; ulcerated and furred tongue; often great thirst ; constipation.

Ulcer of the stomach is a much more common disease than is generally supposed. Many cases thought to be merely neuralgia of the stomach are really chronic ulcer, the two diseases being very easily confounded. 
The ulcer may be very small in size, not more than one fourth of an inch in diameter, or it may extend until it becomes as large as the palm of the hand. Sometimes the ulcer encircles the stomach like a band.

The chief causes of ulceration of the stomach are the use of condiments and other exciting foods, the excessive use of meat, the use of alcoholic liquors, overeating, hasty eating, and hyperpepsia (page 264). It sometimes results from gastric catarrh.

Treatment. - In severe cases the stomach should be given absolute rest, the patient being kept in bed, and nourished by nutritive enemata for some days. After a week or two a liquid diet may be employed, the patient being given only very small quantities of food at intervals of three or four hours. The liquid diet should be adhered to for some weeks, until all the symptoms have disappeared. The severe pain of ulceration may be greatly relieved by fomentations. In some instances ten or fifteen grains of soda taken just before eating afford relief by decreasing the acidity of the gastric juice. In cases of nlceration of the stomach in which dilatation exists, the stomach-tube may sometimes be used to advantage (46) when fermentation is present, but it must be employed with very great care. Salt should be omitted from the solution used in washing out the stomach. The measures elsewhere recommended for hypopepsia (page 260) should be employed, in addition.

Cancer of the Stomach. - The symptoms of cancer of the stomach are pain at the pit of the stomach of a burning or gnawing character, increased by food; tenderness to pressure over the stomach; nausea and 
frequently vomiting, the vomited matters often resembling coffee grains ; hard, pulsating tumor felt near the pit of the stomach; great emaciation; tawny yellow complexion ; symptoms of enlargement of the stomach ; great exhaustion; swelling of the ankles; sometimes general dropsy.

Cancer of the stomach is one of the most frequent forms of malignant disease. Recent researches seem to show that cancer is a parasitic disease. Like other parasitic maladies, cancer does not attack a healthy organ, but only one which has become weakened by abuse. The use of alcoholic liquors and chronic indigestion are doubtless predisposing causes of cancer. The disease itself is not hereditary, but the predisposition to it is. Cancer of the stomach frequently occurs subsequently to cancer in other organs of the body.

Treatment.- This disease is practically incurable. By careful treatment, however, much can be done to prolong the patient's life. The same measures should be employed as have been recommended for chronic catarrh of the stomach, ulcer of the stomach, and dilatation. Dilatation of the stomach occurs in cases of cancer in which the disease attacks the pyloric portion of the organ, thus causing obstruction at the outlet of the stomach. The disease is greatly aggravated by the use of meat. An aseptic dietary (Diet List No. 1) is always indicated. When a state of nlceration is reached, the diet must be wholly liquid in character, and the treatment should be such as has been elsewhere recommended for "Ulcer of the Stomach" (page 276). "General Nutritive Treatment" (page 331) shonld be employed. 
Constipation. - The causes of this exceedingly common condition are very numerous. Some of the most frequent may, be enumerated as follows:-

1. Prolapse of the bowels, a condition in which the stomach is usually involved, is the cause of chronic inactivity of the bowels in a very large number of cases, especially among elderly people. The usual canses of prolapsed bowels are two :-

(1) Tight-lacing from corset-wearing or the pressure of tight bands, in women; or from wearing belts, a practise not uncommon among soldiers and certain classes of laborers, in men.

(2) A relaxed condition of the abdominal muscles, common in both sexes, but most frequent in women, being the result of improper dress, a sedentary life, or inattention to the proper attitudes in sitting, standing, and walking. When the large intestine becomes prolapsed, it is not infrequently folded upon itself, producing a sort of pseudo-stricture, so that the fecal matters, detained in their passage along the intestine, become hardened, and accumulate.

2. Dilatation of the large intestine is usually the result of overaccumulation of fecal matters, but is sometimes caused by abnormal fermentations. This condition may be the result of the preceding, as the pressure of vigorous abdominal muscles is necessary to prevent overaccumulation of gas, and to support the thin walls of the intestines. It is quite likely, however, to result from neglect to empty the bowels regularly. When overaccumulation has been allowed to exist habitually for some months or years, the walls of the intestines may become so stretched that their 
natural muscular activity is gone, and can never be recovered, although some improvement may be secured by proper treatment. This condition is common in both sexes, but is more frequent in women, and is the direct result of improper dress and sedentary habits.

3. Dilatation of the stomach, a condition exceedingly common in persons suffering from dyspepsia, and existing in fully one half of all cases of this kind, sometimes becomes a cause of constipation by provoking intestinal catarrh through the irritating influence of fermented and improperly digested substances, which, after a long delay, escape from the stomach into the intestine.

4. General weakness and relaxation of the muscular system of the body may induce a similar condition of the intestinal muscles, resulting in constipation from deficient peristaltic actirity; hence exhausting labor or, in fact, anything which exhausts the nerve centers, may give rise to intestinal sluggishness.

5. A torpid liver, by which is meant a liver, which does not secrete a sufficient quantity of bile, may be the cause of constipation. Bile is a natural laxative, serving to stimulate the muscular contraction of the intestinal walls. When lacking in quantity, deficient intestinal activity is a natural result. Anything which clogs the liver, or renders it inactive, may be a cause of constipation. The use of animal food, condiments, tea and coffee, and an excessive amount of sweets and fats, must all be considered as productive of constipation, through their influence upon the liver.

6. Hemorrhoids may be a cause of intestinal inactivity, acting mechanically to prevent complete evacua- 
tion of the bowels, and to cause a retention and hardening of fecal matter. The number of persons who carry about with them constantly considerable quantities of hardened fecal matters in the large intestine, must be very great, judging from the results of treatment in dislodging old accumulations of this sort in cases which have come under the writer's care. In some cases, great quantities of black and decomposing matter have been discharged after two or three weeks, during which time the patient's bowels had been daily washed out with two or three quarts of warm water applied in the most thorough manner possible.

7. Rectal ulcer, catarrh of the rectum, and an irritable rectum resulting in spasmodic contractions of the muscles which close the lower end of the bowel, sometimes induce constipation by presenting too great resistance to the expulsive effort by which the fecal matters are discharged from the body, so that the bowel is never completely emptied, and the accumulations thus begun are gradually increased.

8. A loss of natural sensibility in the mucous membrane of the rectum is doubtless a frequent cause of constipation. This semi-paralyzed condition is usually due to failure to evacuate the bowels at a regular time. After long neglect of this sort, the natural reflex activities by which the bowels are stimulated to expulsive action are no longer awakened by the presence of fecal matters in the rectum; and constipation is the result.

9. Another cause of constipation is excessive dryness of the fecal matters, which prevents their ready movement along the large intestine. This is usually 
the result of too long retention within the lower bowel, but may result from other causes, as a fererish condition, or from a deficient secretion of the mucus which acts as a lubricant.

10. Nany persons are suffering from chronic constipation as the result of "orificial surgery." The rectum not only contains pockets in which a quantity of mucus is formed for the purpose of lubricating the fecal mass as it is expelled from the body, but a fringe of papillæ is also found in the healthy rectum just within the anal orifice. These papillæ are connected with nerves, the function of which is to bring forcibly and involuntarily into action at just the right moment the strong muscles of the abdominal walls, so as to accomplish complete eracnation of the bowels. These pockets and papillæ were discovered by Professor Horner, the cminent anatomist of Philadelphia, who described and pictured them in his "Anatomical Atlas" nearly half a century ago. The "orificial" surgeon often industriously trims off every papilla, and slits up every pocket, for no other reason, apparently, than to line his own pockets, and certainly to the great disadvantage of the patient, who, with his rectum thus maimed, has lost two important links in the chain of automatic activities by which nature secures a daily eracuation of the bowels.

Treatment.-Any course of treatment, to be curative of this condition, must take into consideration all the varions possible causes which may have brought about the intestinal inactivity in any particular case. It is necessary also, not only to remove these causes, but to repair, so far as possible, by the application of 
efficient means, the damage which has been wrought by wrong habits and morbid influences.

The matter of first importance in the treatment of constipation is the diet. The abundant use of fruit is one of the most excellent means of preventing and curing this disease. One or two oranges before breakfast; a couple of apples at breakfast; the free use of steamed figs, stewed prunes, and other fruits, are means to be recommended in nearly all cases of chronic constipation. There are, of course, some cases in which fruits must be avoided. In these cases coarse grains cracked wheat, oatmeal, graham or bran bread, bran cakes, etc.- serve a useful purpose. Peas, beans, lentils, asparagus, green peas, string beans, and similar vegetables which are easy of digestion, but which contain a considerable amount of woody or indigestible substance, may also be advantageously used. Coarse vegetables, howerer, must be aroided in cases where there is marked dilatation of the stomach. Granose, gofio, and other excellent health foods manufactured by the Battle Creek Sanitarium Health Food Co., Battle Creek, Mich., have proved of very great value to thousands of persons suffering from this condition. Granose (Diet List No. 25), a new health food recently perfected, is especially valuable as a food-cure for constipation, if freely used. Most persons are very promptly relieved by its use. Bromose (Diet List No. 25), another new health food, is also found to be a very excellent remedy in these cases.

A glass of cold water before breakfast is a prescription which has cured many cases of constipation. The free use of water, either hot or cold, taken one or two 
hours before each meal, is a valuable remedy. (See Diet List No. 21.)

Exercises of various kinds, particularly such as bring into active play the muscles of the lower part of the trunk, are essential in the treatment of many cases of intestinal inactivity. Walking three to five miles a day is sufficient to secure regularity of the bowels in many persons. A short walk before breakfast is especially helpful.

The exercises of the Ling system, known as Swedish gymnastics, have been found of special value in the treatment of this class of cases at the Battle Creek Sinitarium (43). Horseback riding, rowing, and bicycle riding are also very helpful.

Massage of the bowels $(\mathbf{2 9}, \mathbf{3 0})$, Swedish movements (43), the wet girdle (9), applications of electricity to the abdomen $(66,69)$, and, in special cases, to the abdomen and rectum, are measures of great importance. The liver douche (20) and the hot and cold full bath (21) may also be employed. General tonic measures (page 333) are essential as means of reinforcing the general nervous energy. In cases of prolapse of the bowels, the abdominal supporter (45) should be worn.

Introduction into the rectum of a small quantity of cold water, half a pint or a pint, before breakfast, to be retained until after breakfast, is a useful measure. A small, cold enema taken at the regular time for the bowels to more, is better than a large, warm one, as it is a more powerful stimulant to intestinal activity. A small amount of cold water introduced into the rectum at night upon retiring, is a useful measure in cases where 
the intestinal contents are dry and hard; half a pint or a pint is a sufficient amount. In some cases in which the stools are large and the rectum irritable, an ounce or two of olive or almond oil introduced at night or before breakfast is a useful measure. Camphor water, consisting of three or four ounces of water to half a teaspoonful of spirits of camphor, may be introduced into the rectum before breakfast with advantage in many cases. Some cases are relieved by the introduction of a small quantity of glycerin,- two or three tablespoonfuls with an equal quantity of water,-although sometimes pure glycerin may be necessary. Two or three trials will determine. Suppositories made of glycerin or glycerin and camphor are also valuable for the same purpose ; they may be introduced either at night or before breakfast, or at both times.

When the rectum is the seat of catarrh, a mixture consisting of equal parts of starch and boracic acid, introduced by means of a proper instrument, is a very helpful measure. The use of equal parts of boracic acid and subcarbonate of bismuth, or subcarbonate of bismuth alone, is preferable when there is an unusual degree of irritation. The introduction of a dram or two of boracic acid into the rectum daily before breakfast, is a most valuable remedy, and one which is often found curative of this condition.

It must not be forgotten that regularity in attending to the demands of nature is a matter of the utmost consequence in these cases, both as a preventive and a curative measure. Sometimes the inability to evacuate the bowels is due to weakness of the abdominal muscles; in such cases it may be necessary to aid the bowels 
by pressure of the hands. Several cases have come under the author's care in which there was inability to evacuate the bowels when sitting in the usual position, but no difficulty when a crouching position was assumed. This was doubtless due to the increased pressure brought to bear upon the abdominal contents when sitting in a crouched position.

Some cases of constipation tax the skill and ingenuity of the physician to the utmost, and cannot be relieved by the simple measures which can be undertaken at home. There is now and then a case in which extreme dilatation of the colon exists, so that this organ entirely loses its power to contract upon itself, and becomes little more than a lifeless sac. In such cases constant use of the enema (22) or coloclyster is the only means by which the bowels can be relieved, and this measure must be employed habitually.

There is no one remedy for constipation so valuable in all cases as a diet consisting chiefly of granose, fruits, and nuts or bromose (Diet List No. 25). 


\section{REMEDIES FOR THE HOME TREAT- MENT OF DYSPEPSIA.}

Is the majority of cases of chronic dyspepsia the patient is more or less permanently disabled, and hence needs to be made familiar with measures of treatment which he can himself employ at home, since he cannot have a physician or nurse always with him, and cannot live continually at a sanitarium. There is no way in which the chronie sufferer from indigestion can so well accomplish this object as by placing himself for a few months under treatment at a thoroughly equipped and rationally conducted sanitarium. In such cases health is to be obtained only by a long and persevering course of correct regimen and careful health culture and training, which, in order to secure the best results, requires the personal supervision of a skilled physician and of trained assistants in carrying out the necessary treatment. As it is impossible, however, that all should enjoy these advantages, the writer has undertaken to describe such measures of treatment as extensive observation and study in the hospitals of this country and Europe, and many years' experience in the treatment of various forms of digestive disorders, has led him to consider most efficient and best adapted for employment at home.

Before beginning a course of treatment, the patient should read carefully the chapter" on "Symptoms," and 
make up his mind as to the form of dyspepsia from which he is suffering. The diagnosis being determined, he should give careful attention to the prescription for the treatment of the particular form of dyspepsia from which he is suffering, and should arrange his diet, program of treatment, and whole course of life with reference to the requirements of his malady, no matter how inconvenient it may be to do so. Nothing less than thorough measures will accomplish anything in the treatment of this disease.

It must be remembered, also, that a malady which has been acquired as the result of long years of transgression of the laws of health, cannot be overcome by a few days or even a few weeks of treatment. Many months of persevering effort are usually required to secure permanent and farorable results, although it is just and proper to expect some immediate amelioration of distressing symptoms when they exist.

Not infrequently there is a slight exaggeration of symptoms at the beginning of treatment, and sometimes new symptoms make their appearance. There is often a slight or even a considerable loss of flesh for a short time. Any aggravation of symptoms should be carefully considered. The cause may be a mistake in diagnosis, incorrect application of the treatment, or some individual idiosyncrasy which must be taken into con, sideration. As a rule, any measure of treatment or regimen which aggravates symptoms should be at once, discontinued.

A reasonable amount of good judgmenț and common sense must be employed in connection with the treatment recommended in order for it to be fully effect- 
ive. Most of the measures described in the following paragraphs are designated by numbers, for convenience of reference :-

1. The Sponge.or Hand Bath. - Soft water, a soft sponge or a linen or cotton cloth, and one or two soft towels or a sheet, are the requisites. The hand may be used in the absence of a cloth or a sponge for applying the water. For a saline sponge bath add a tablespoonful of salt to a quart of water. One part of vinegar may be added to three parts of water for a vinegar sponge bath.

The temperature of the bath should not be above $92^{\circ} \mathrm{F}$, and $85^{\circ}$ is generally better. Most people can habitually employ a temperature of $75^{\circ}$ or $80^{\circ} \mathrm{F}$. without injury, and some receive most benefit from a still lower temperature. The use of a much lower temperature is not commonly advisable, however, and is often productive of great injury.

Begin the bath by wetting the head. Bathe the face, then the neek, chest, shoulders, arms, trunk, and back. Rub vigorously until the skin is red, to prevent chilling; for even when the temperature of the room is nearly equal to that of the body, the rapid evaporation of water from the surface will lower the external temperature very rapidly, unless a vigorous circulation is maintained.

After thoroughly bathing the upper part of the body, turn the attention to the lower portion, continuing the rubbing of the upper part at brief intervals to prevent chilliness. As soon as the bathing is concluded, envelop the body in a sheet and rub dry, or dry the skin with a towel. When the surface is nearly 
or quite dry, rub the whole body vigorously with the bare hand.

The bath should not be prolonged more than ten or fifteen minutes. Five minutes is a sufficient time to secure all the benefits of the bath, and even three minutes will suffice.

Persons who chill easily will find it better to bathe only a small portion of the body before drying it. Some persons will even find it necessary to retain some of the clothing upon the lower part of the body while bathing and drying the upper part.

This bath may be given to a feeble patient with very little disturbance, even in bed. Only a small portion of the body should be uncorered at one time, that being bathed, dried, and rubbed, and then covered while another part is treated in a similar manner.

2. The Full Bath. - This bath is taken in an ordinary bath-tub, which should contain water enough to cover the body. For a hot bath a temperature of $100^{\circ}$ to $105^{\circ} \mathrm{F}$. should be employed ; for a warm bath, $90^{\circ}$ to $95^{\circ} \mathrm{F}$. When employed for elininative purposes, the bath should be continued until the patient perspires gently. A full bath, accompanied by the use of soap and the flesh-brush, is especially useful as a cleansing bath. Care should be taken to lower the temperature of the bath fifteen or twenty degrees just at its conclusion, to prevent the patient frơm taking cold. The body should be thoroughly dried after the bath in this as in all other baths. In cold weather, oil should be applied after the use of soap, to lessen the danger of taking cold. 
3. The SaIt Glow. - This is a most excellent tonic bath. It consists essentially in rubbing the whole body with moist salt, applied freely to the surface. The bath should not occupy more than three to five minutes. The salt should be rinsed off by pouring a

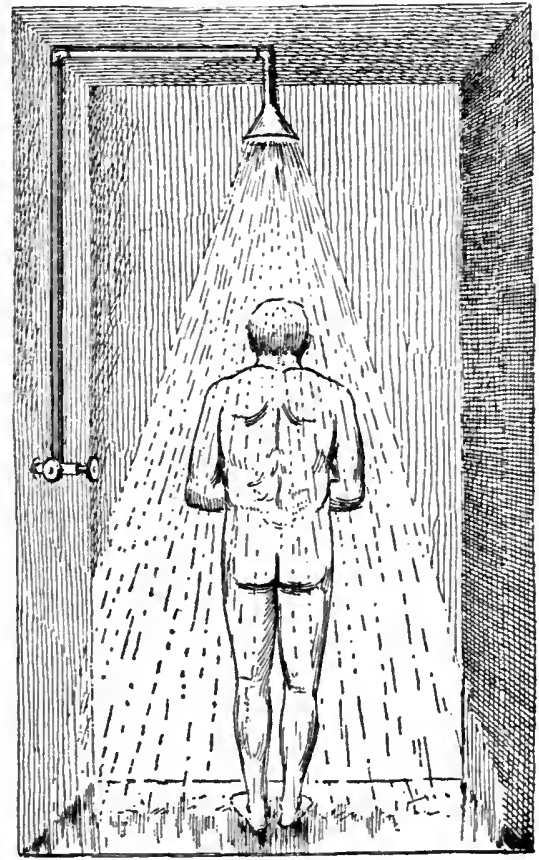

Fig. 19.- Shower Bath.

pail of water over the patient, by a shower bath, or by sponging.

4. The Shower Bath. - An apparatus such as is indicated in Fig. 19 is the most convenient for this bath, but water poured through an ordinary colander, or the simple device shown in Fig. 20 may be employed. 
This consists of a vessel with a perforated bottom, furnished with a hollow tube for a handle. The vessel is filled by immersing it in water, and the thumb placed over the end of the handle, which prevents the water from running out. When the thumb is removed, the water falls through the perforated bottom in a shower. The shower bath is usually administered cold or at a temperature of $60^{\circ}$ to $80^{\circ} \mathrm{F}$. It should last but a few seconds. This is a most powerful tonic bath. The

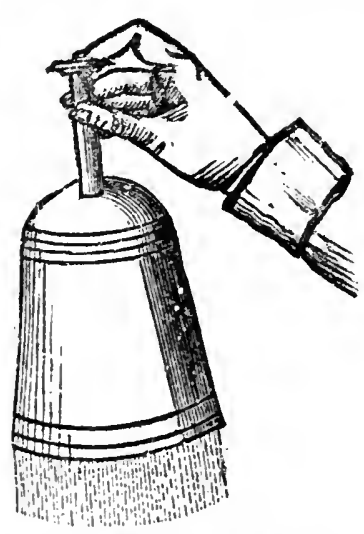

shower may be applied to the whole body, at a single point, as over the region of the stomach and liver, or to any portion of the trunk or limbs.

The temperature shonld be $60^{\circ}$ to $80^{\circ} \mathrm{F}$. for tonic effects. In taking a general shower bath, the patient, properly prepared, begins the bath by placing one hand Fig. 20.-Stmple Shower Batr. or one foot under the shower, and then quickly passing one arm under to the shoulder, then the other arm, allowing the water to fall upon the chest, then upon the back, and so on until the whole surface has been exposed to the direct force of the stream.

5. The Rubbing Wet.Sheet. - This is a very valuable means of stimulating the skin, and, through it, the central nervous system; indeed, it is one of the best of all tonics. The patient, standing, is quickly wrapped with a sheet wrung ont of water at a tempera- 
ture of $80^{\circ}$ to $90^{\circ} \mathrm{F}$. for feeble patients, and a lower temperature for stronger ones. $\mathrm{He}$ is then briskly rubbed outside the sheet. The sheet may be renewed from one to three times, according to the strength of the patient or the intensity of the effect desired (Fig. 21).

6. The Wet.Sheet Pack. - Two or three comfortables or thick blankets, one woolen blanket, and a large linen or cotton sheet, are the articles necessary. It is important that the sheet be sufficiently large to extend twice around the patient's body. Two or more blankets are required in cool weather or by weak patients. Spread the comfortables, one by one, upon a bed or straight lounge, making them even at the top. Over them spread the woolen blanket, allowing its upper edge to fall an inch or two below that of the last comfortable.

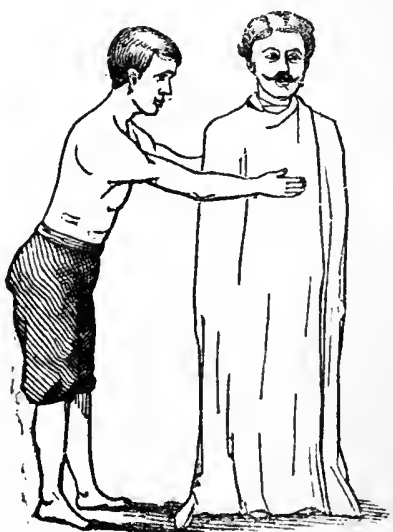

VIg. 21.-RUBBING WET-SHEET.

Wet the sheet in water of the proper temperature, having gathered the end in one hand so that it can be quickly spread out. Wring so that it will not drip much, place its upper end even with the woolen blanket, and spread it out on each side of the middle sufficiently to allow the patient to lie down upon his back, which he should do quickly, letting his ears come just above the apper border of the sheet, and extending his limbs near together. Carefully wrap the patient, first with 
the sheet and afterward with the blanket, taking pains to exclude the air (Fig. 22).

7. The Trunk Pack. - This is administered in the same way as the wet-sheet pack, with the exception that the application is confined to the trunk of the body.

8. The Hot and Cold Trunk Pack. - This valuable measure is administered in the same manner

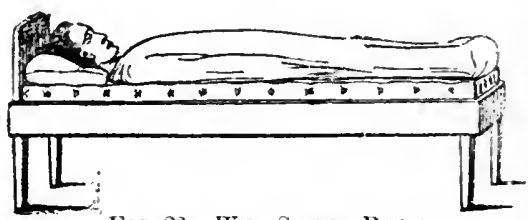

Fig. 22.- Wet-Sheet PACK. as the truik pack, except that a rubber lag tro thirds full of hot water is placed orer the stomach before the cold sheet is applied. A flamel cloth should be placed next the skin to prevent injury from the hot bag.

9. The Wet Girdle. - This was a favorite remedy with the early German hydropathists, and it is a very useful appliance when properly employed, though it has been much abused by excessive use. To apply it well, a coarse towel about three yards long is the most convenient. Wet one half of this in cold water, wring until it will not drip, and apply it to the abdomen, placing one end at the side and bringing it across the front first, so that two thicknesses of the wet portion will cover the abdomen. After winding the whole tightly around the body, fasten the end securely with pins or with tapes attached for the purpose. Cover all with several folds of flannel.

In cold weather, the moistened towel should be covered with oiled muslin before the application of the flannel, and care should be taken to have the dry wrap- 
pings extend at least two inches beyond the edges of the moist towel, to prevent evaporation and chilling. The colder the water in which the towel is wrung, the more vigorous will be the effect produced. For feeble paticnts the towel should be wrung as dry as possible.

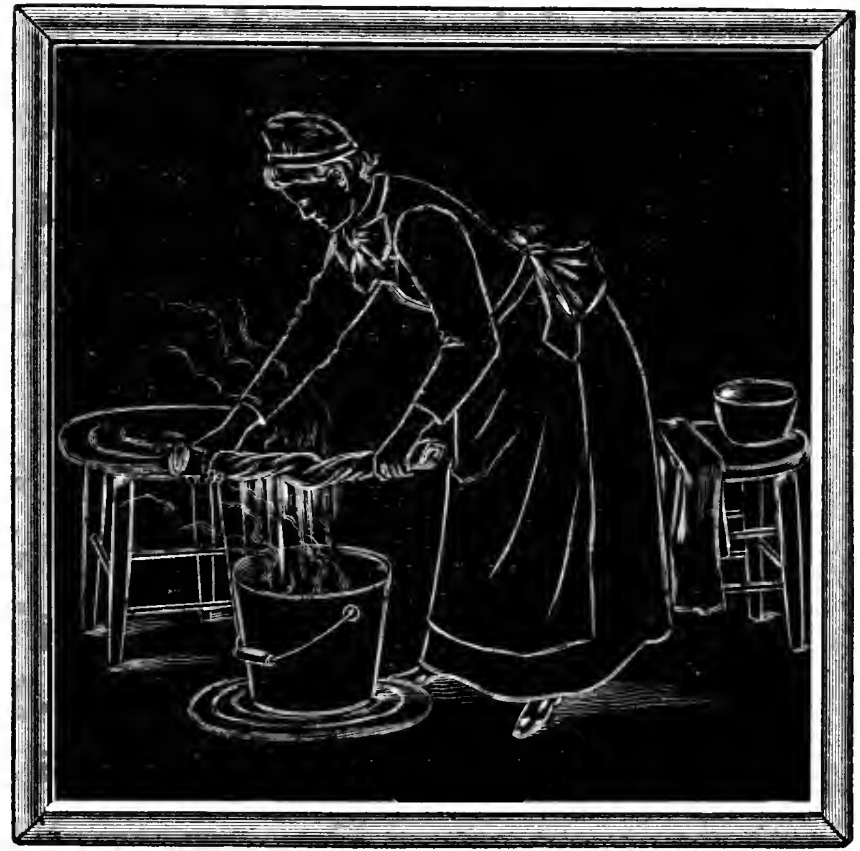

Fig. 23.- Fomentation.

10. Fomentation. - Fold a soft flamel twice, so as to make four thicknesses. Dip in very hot water, lifting out by the corner and placing in the middle of a towel. Roll up quickly in the towel lengthwise, and wring nearly as dry as possible by twisting the ends of the towel. In this way the fomentation can be wrung 
out much hotter than with the hands. Of course it will be too hot to apply to the bare flesh; but the skin

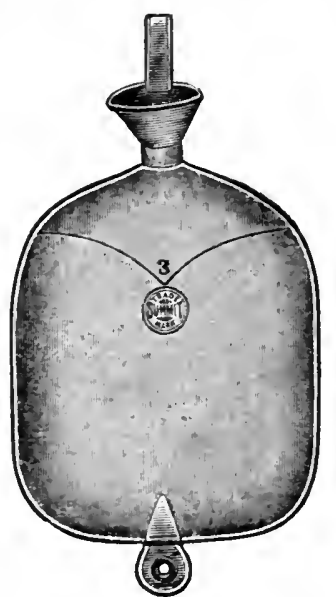

Fig. 24.-Пот Water-BaG. should be protected by one or more thicknesses of flannel, and the fomentation applied at once, and covered with another dry flannel. Renew the fomentation when the heat begins to moderate very perceptibly, and continue as long as necessary (Fig. 23).

\section{Mustard or Turpen.} tine Fomentation.-The effect of the fomentation may be increased by adding a tablespoonful of mustard to the water from which the fomentation is wrung, or by sprinkling over the fomentation cloth when ready to apply, an ounce of alcohol in which a teaspoonful of turpentine has been dissolved.

12. Dry Heat. - Dry applications of heat may be made by means of a rubber bag filled with hot water (Figs. 24, 25), heated bricks wrapped with flannel

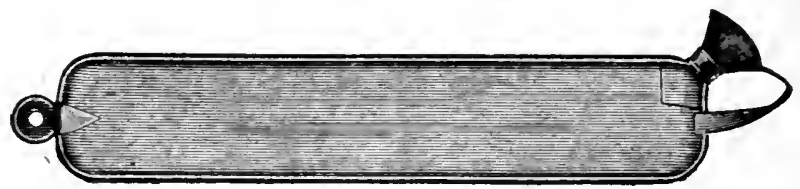

Fig. 25.--SPINE BAG.

cloths, flannel bags containing hot salt, bottles filled with hot water, and similar means. 
13. The Foot Bath. - Any vessel sufficiently large to receive the feet and enough water to cover them to the ankles, is suitable for this bath. The temperature should usually be $100^{\circ}$ to $105^{\circ} \mathrm{F}$. If the water is cold, it should not be more than one fourth of an inch deep.

The alternate hot and cold foot bath is a very valuable remedy for cold feet. It is given thus: Place the feet in hot water $-100^{\circ}$ to $110^{\circ} \mathrm{F}$. - for two or three minutes. Then withdraw them, and plunge them quickly into a bath of cold water, $60^{\circ} \mathrm{F}$. or less. After two or three minutes, restore them to the hot bath. Thus alternate three or four times, and conclude by dipping the feet quickly into the cold water and wiping dry. This bath produces a most powerful reflex action (Fig. 26).

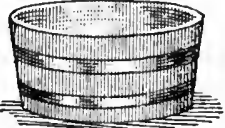

14. The Sitz or Hip Bath. - For this bath a common tub may be used, by placing a support under one edge to elevate it two or three inches; but it is better to use a tub made for the purpose, which should lave the back raised eight or ten inches higher than the front, to support the back of the patient, and the sides sloping gradually so as to support the arms of the bather. The bottom should be elevated two or three inches. The depth in front should be abont the same as that of a common wash-tub.

Enough water is required to cover the hips and extend a little way up on the body. Four to six gallons is about the proper quantity.

A very good plan for administering this bath, and one which will be applicable to most cases, is this: 
Begin the bath at $92^{\circ}$ or $93^{\circ} \mathrm{F}$. If a thermometer is not at hand, pour into the bath-tub three gallons of fresh well- or spring-water, and then add one gallon of

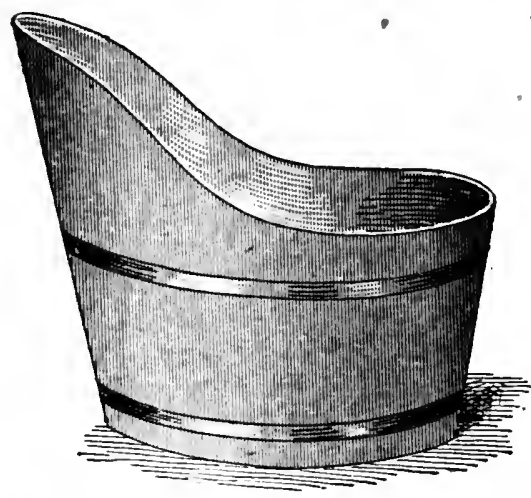

Fig. 27.-The Sitz Bath TeB.

boiling water. This will give the desired temperature. After the patient has been in the bath ten minutes, cool it down to $85^{\circ} \mathrm{F}$., which may be done by adding a gallon of cold water. Continue the bath five minutes longer; then administer a pail douche or spray at about $85^{\circ} \mathrm{F}$, and wipe dry, as directed after a hand bath (Fig. 27).

15. The Sun Bath. - The whole body, unprotected by clothing, should be exposed to the sun, the head only being covered. The exposure should be from three to fifteen minutes, when a tepid sponge bath should be administered.

16. The Vapor Bath. - This excellent eliminative bath may be easily improvised by placing the patient in a chair surrounded by thick coverings, and heating the water in a pail or small tub placed beneath the chair by gradually lowering into it hot stones or bricks. For permanent use, a wooden box may be constructed after the plan shown in Fig. 28.

The skin of most dyspeptics is very inactive, and with many the perspiration has an offensive odor. On 
this account, a short hot-water or vapor bath may be taken once a week with advantage. The bath should be continued just to the point of inducing perspiration, but not longer, as warm baths are relaxing and debilitating. A dash of cold water over the body, poured from a pail or by means of the shower, should immediately follow the hot bath. The patient should then be quickly dried and rubbed, so as to secure a good reaction. Wrapping in a dry woolen blanket promotes reaction, and is necessary in the cases of some thinblooded dyspeptics. A full hot bath, an improvised vapor or hotair bath, or a hot-blanket pack may be used as a means of inducing gentle perspiration. The best of all such measures, however, is the sun bath or the electric-light bath.

\section{The Hot-air Bath.-}

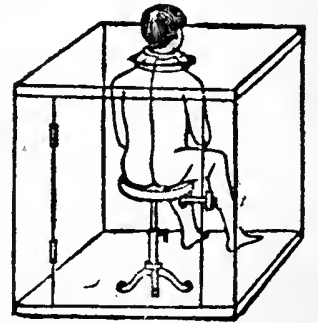

Fig. 28.-VAPOR BATH.

This is administered in a manner similar to the vapor bath, except that the heat is produced by burning alcohol in a small dish placed for safety in a ressel containing a little water. The purpose of the hot-air bath is to induce perspiration. A cold shower or sponge bath should be applied at the conclusion of the bath.

18. Heat and Cold to Spine. - Alternate fomentations and cold compresses may be applied, or alternate hot and cold sponging, if it is preferred, - the alternations being made every half minute, or once in one to three minutes. Water as hot and as cold as can be burne should be used. In some cases it is necessary to employ ice in order to obtain 
the desired effect. When ice is applied to the skin, the cold application should occupy only five to ten seconds.

19. The Hot and Cold Pour, or Pail Douche. - Two pails of water, one at a temperature of $120^{\circ}$ to $130^{\circ} \mathrm{F}$, the other as cold as can be obtained,- ice water when a very strong effect is desired,-- and a dipper, are the essential requirements. If the application is to the spine, the patient should sit on the edge of the tub while the water is poured upon the spine from a dipper. The force of the application may be raried somewhat by the height at which the dipper is held. The application should be alternated every eight or ten seconds.

20. The Hot and Cold Liver Douche. The patient lies in a full-bath tub while a hot and cold pour is administered to the right side, as directed above. The application should continue five to fifteen minutes. If the patient chills easily, the tub may contain a sufficient amount of warm water to partially immerse the body.

In cases of hypopepsia and apepsia not accompanied by tenderness in the region of the stomach, this douche may be employed with adrantage as a means of stimulating the activity of the stomach glands. The patient should lie in such a position that the water will fall upon a point just below the end of the breast bone. The best time for the liver or stomach douche is just before retiring at night, or an hour or two before dinner. A heating compress, or wet girdle (9), should be applied afterward, and worn over night or during the digestion of the meal, in obstinate cases, 


\section{The Hot and Cold Full Bath.-The} full bath is prepared in the usual manner, with a temperature of $100^{\circ} \mathrm{F}$. After lying immersed in water for about one minute, the patient rises to a sitting posture. A quantity of cold water at a temperature of $60^{\circ}$ to $70^{\circ} \mathrm{F}$. is dashed about the neck and shoulders. The patient then lies down in the bath for a minute or two, the water being warmed again. He then sits erect while the cold application is made as before. The bath may be continued from one to five minutes, concluding with a cold application and vigorous rubbing.

Another method is to administer a hot bath at a temperature of $100^{\circ}$ to $105^{\circ} \mathrm{F}$. for five or six minutes, or until the skin is reddened. The patient is then taken from the bath, and the surface rubbed with a towel wrung out of ice-water, or

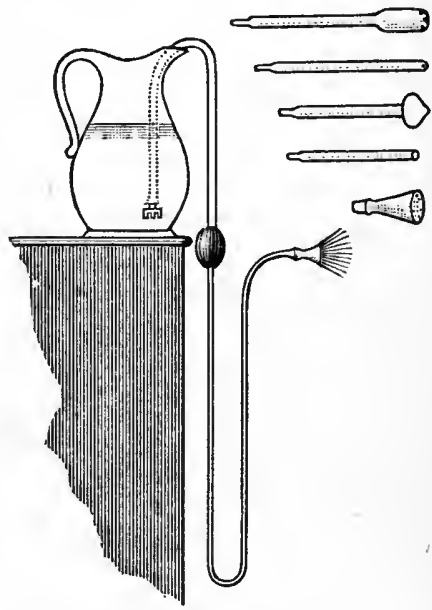

Fig. 29.-SyPhoN SYrixge. with a piece of ice. The cold rubbing should be executed very rapidly. Lastly the patient should be rubbed dry with a coarse towel.

22. The Enema. - This exceedingly useful application of water is best administered by a syphon or fountain syringe (Figs. 29, 30), A bulb syringe may be employed, but there is always danger of pumping air into the intestines, and thus giving rise to severe pain, 
The patient should lie upon the back. The temperature of the water should be $95^{\circ}$ to $100^{\circ} \mathrm{F}$. When the purpose is evacuation of the bowels, a small cold enema is useful as a means of stimulating the lower bowel. A large hot enema is employed in cases of catarrh or for the relief of pain.

23. The Coloclyster. - This is simply a modified form of the enema. The patient should lie upon the right side while the water is being introduced, or should assume the knee-chest position. In the kneechest position, the patient first kneels, then bends for-

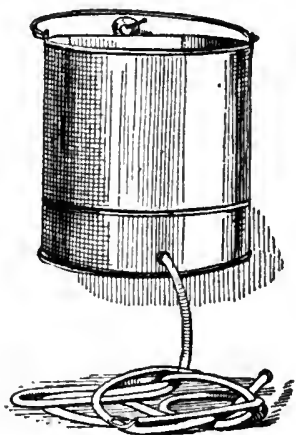

Fig. 30.-Fountain SYRINGE.

ward until the head and chest are on a level with the knees. The thighs should be upright, so that the thighs, trunk, and the surface upon which the patient is kneeling shall form a right-angled triangle. The purpose of this application is to render possible the introdnetion of a larger quantity of water into the colon. Three or even four quarts can be thus introduced in some cases.

\section{The Cold and the Graduated Enema.} - The cold enema is a powerful means of stimulating intestinal activity. The quantity of water used should not be greater than half a pint in ordinary cases.

The graduated enema consists of an ordinary enema gradually cooled from day to day by the addition of half a pint of cold water, the total amount of water employed being at the same time diminished. The amount of water used each day should be lessened 
sufficiently so that by the end of ten days or two weeks the total quantity used will not be more than a pint, and the temperature $60^{\circ}$ to $70^{\circ} \mathrm{F}$. In a few days more, if proper diet and other measures are employed, the enema may be discontinued.

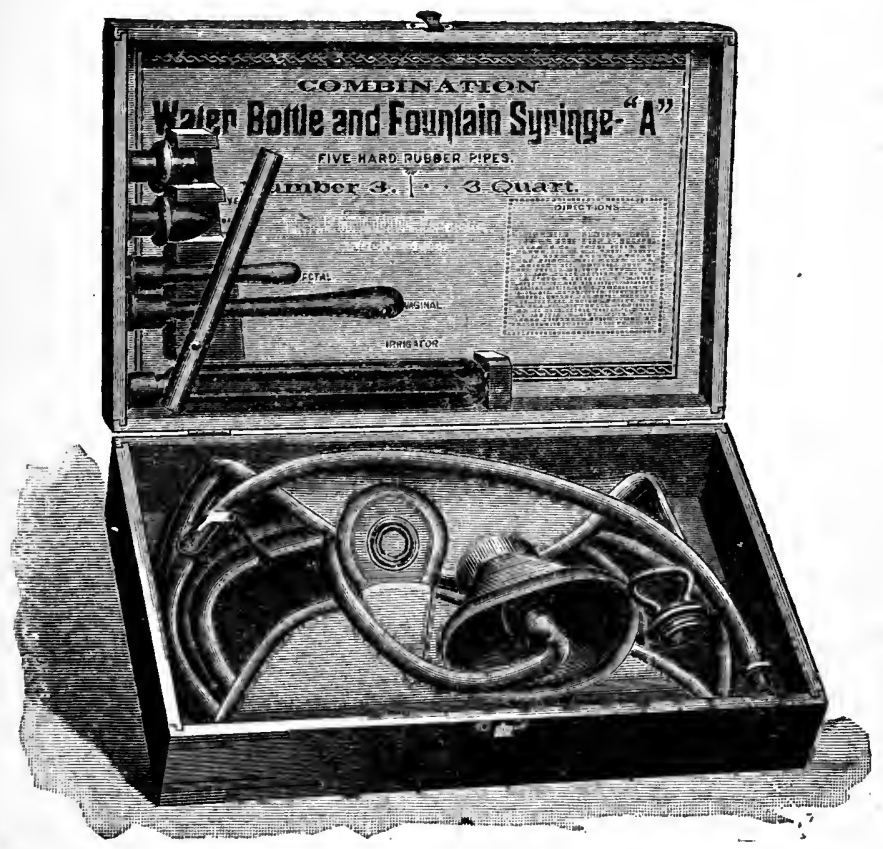

Fig. 31.-Water Bottle and Syringe Combined.

25. The Laxative Enema. - In cases of extreme constipation it is sometimes necessary to employ a laxative enema. Strong soapsuds is excellent for this purpose, and is effective in most cases. It is sometimes necessary to add common salt to the water 
in the proportion of a tablespoonful to the quart. The following mixture may also be advantageously employed in very obstinate cases : Sulphate of magnesia, one tablespoonful; glycerin, one tablespoonful ; boiling water, two tablespoonfuls. The mixture should be introduced as high up in the bowel as possible, a catheter being used for the purpose if necessary.

26. The Oil Enema. - Two or three ounces of olive oil introduced into the rectum at night serves as a sufficient remedy for constipation in certain cases. The oil enema may also be advantageously used in cases in which hardened masses of fecal matter have accumulated in the intestine, and cannot be removed by the ordinary warm-water enema.

27. Oil Rubbing. - Applications of oil are always made after certain kinds of baths. Ordinarily, a tepid sponge bath is first given, after which the oil is systematically applied to the entire body with vigorous rubbing. Any excess of oil remaining should be wiped away. Olive oil, cocoanut oil, or even vaseline may be employed.

28. Massage - Kneading. - Superficial and deep kneading, as illustrated in Figs. 1 and 2, Plate VI, are among the most useful procedures employed in massage. The movement is that of firmly grasping and then releasing the tissues. The purpose is to knead and manipulate the skin and the muscles so as to stimulate the circulation and other vital activities in these structures.

29. Abdominal Massage.-(Fig. 3, Plate VI.) The opcrator endeavors to seize the abdominal contents, with both hands, manipulating them precisely as a 

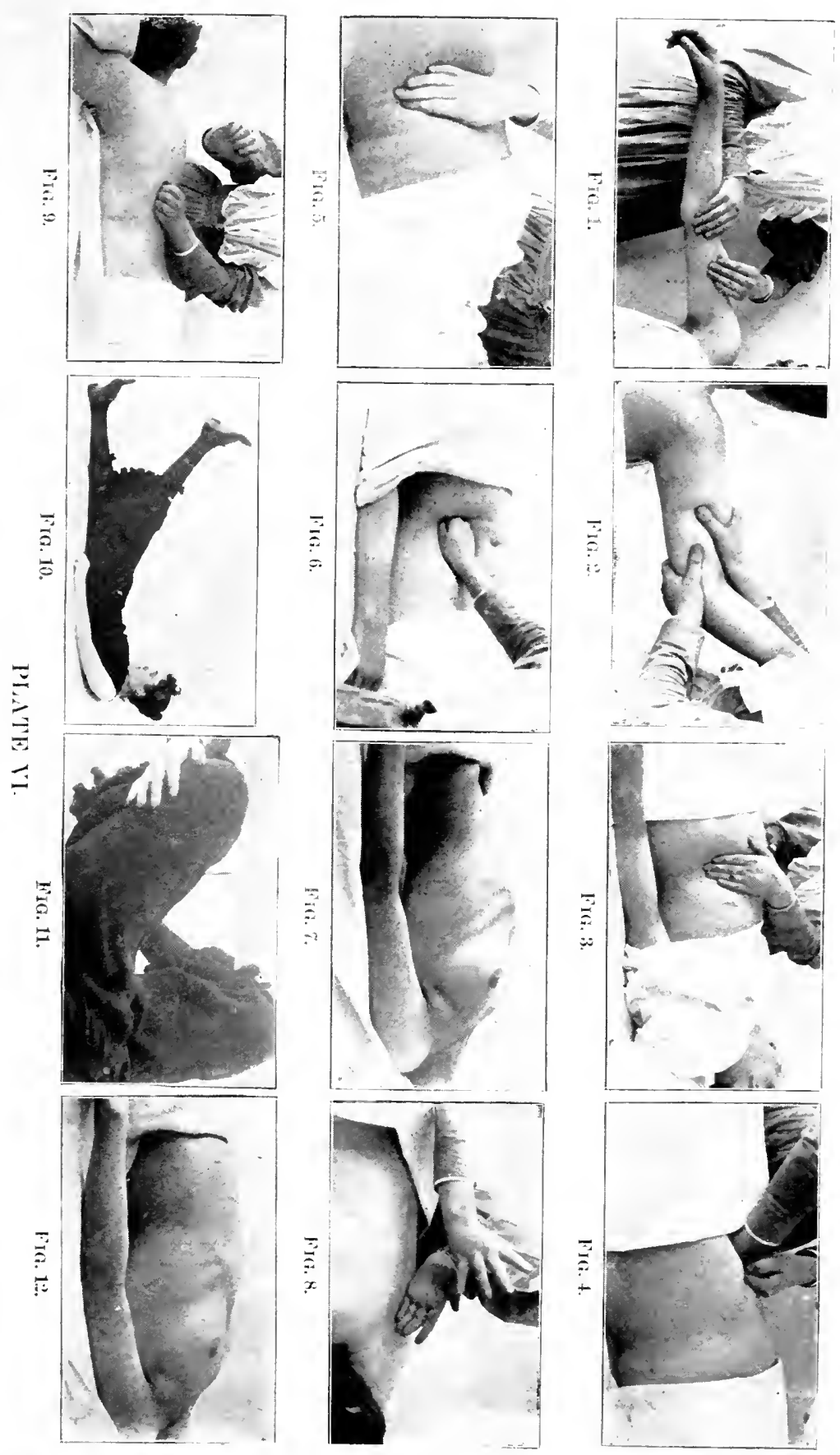


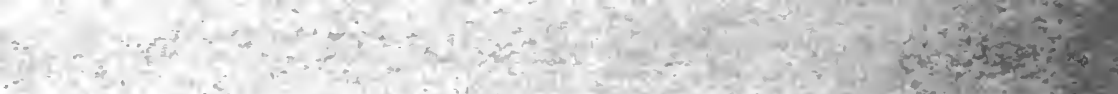

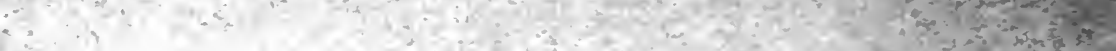
20 (1) $x^{x}=\pi^{2}+n^{2}$

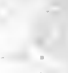


baker does a mass of dough, the fingers of one hand being used in opposition to the heel of the other hand, and the abdominal contents kneaded and manipulated between the two hands. In this procedure the heel of one hand of the manipulator operates upon the side of the patient nearest him, while the fingers of the other hand operate upon the tissues of the opposite side. This method is only applicable to cases in which the abdominal walls are considerably relaxed. This, as well as most other forms of abdominal massage, requires the services of an assistant, the patient lying upon a bed or couch.

30. Massage of the Bowels. - (Fig. 4, Plate VI.) With the closed fists used in alternation, the operator works aiong the whole course of the colon, beginning at the lower end of the cecum, directing the movements upward to the lower border of the ribs on the right side, following the oblique border of the ribs to a point midway between the umbilicus and the sternum, at which the median line is crossed; then down on the opposite side, ending at a point close to the pubic bone, and just to the left of the median line.

If the patient is able, he should make a practise of kneading and percussing the abdomen himself for fifteen or tweenty minutes night and morning. This is a powerful stimulant to muscular activity. Many years ago a quack doctor in New York City made a fortune by treating dyspeptics by this method alone. He put every patient under an oath of secrecy, and required certain wholesome restrictions of diet, which of course aided in the cure, 
31. Massage of the Stomach. - (Fig. 5, Plate VI.) With the patient lying upon the back, the assistant stands upon the right side, with his back to the patient, and executes the following movements : Placing the right hand upon the left side of the abdomen opposite the umbilicus, with the fingers extended and close together, he presses the ulnar border of the hand backward, at the same time carrying it upward with a vibratory movement. Following along under the ribs of the left side, he continues the movement upward to the epigastrium. At this point, before releasing the tissues, he places the tips of the fingers of the left hand so as to support the tissues at the point to which they have been lifted by the movement of the right hand, making firm pressure; then withdrawing the right hand, he repeats the morement. This is continned for three or four minutes. If it is desired to empty the stomach, the strokes should be carried across the epigastrium, and along under the lower border of the ribs of the right side. The following movements are especially useful in aiding digestion :-

Execute the movements just described, and at the same time have the patient breathe deeply: With every alternate breath the patient should execute what the writer terms inspiratory compression, thus: Filling the lungs completely, hold the breath for a few seconds, and at the same time raise the head forward as far as possible, strongly contracting the abdominal muscles.

Gentle kneading of the abdomen, especially its upper portion, is an excellent means of stimulating muscular activity on the part of the stomach, and thus 
preventing too long delay of the food in this part of the digestive apparatus. By this means the local circulation is stimulated, and the natural muscular action of the bowels is both imitated and encouraged. This is an excellent remedy, and may be employed to advantage each night and morning, and for half an hour or more after each meal.

32. Replacement of the Viscera. - (Fig. 6, Plate VI.) The patient lying upon the back, with the head - not the shoulders - elevated, knees well drawn up, the operator places his hands at the lower portion of the abdomen and presses inward, lifting upward at the same time. The patient should relax the abdominal muscles as perfectly as possible, and should take full, deep respirations. With each expiration the operator endeavors to lift the contents of the abdomen a little higher, holding the hands firmly during the inspiration, so as not to lose the ground gained. The patient should take full, deep inspirations, expanding the waist, and lifting the chest well.

\section{Inspiratory Lifting of the Abdominal} Contents. - A number of years ago the author discovered a method by which the abdominal organs may be forcibly lifted into position by a simple modification of the breathing movements. The method is as follows: The patient, lying upon the back, after fully emptying the lungs, executes the same movements as in ordinary inspiration, but, by closing the throat, prevents the admission of air to the lungs. Care is taken to raise the chest as high as possible, and at the same time to draw in the abdominal muscles with as much force as possible. The result is, that, no air being 
admitted to the lungs, the stomach, bowels, and other viscera are sucked up into the chest in a very forcible manner. By grasping the lower abdomen with the hands, and lifting upward at the same time this movement is executed, the effect upon prolapsed organs may be greatly increased. Fig. 7, Plate VI, shows very clearly how this movement is effected.

34. Hacking. - This consists in stroking the part with the erge of the hand, the fingers being separated, as shown in Fig. 8, Plate VI.

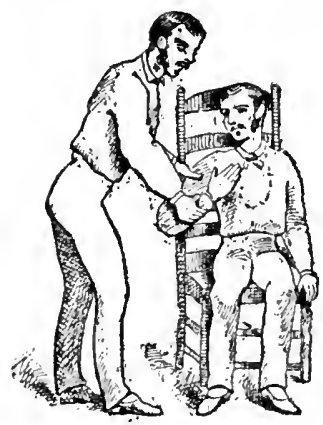

Fig. 32.- Arm Flexion.

35. Beating. - This procedure of massage consists in striking the body with the halfclosed fist, as shown in Fig. 9, Plate VI.

36. General Massage. General massage consists in the rubing and manipulation of the whole body. The movements are chiefly those described as friction and kneading (28). These movements are applied to the limbs and back. Abdominal massage (29) and massage of the stomach and bowels $(30,31)$ are employed for the abdominal region; hacking and beating $(34,35)$ for the spine (Figs. 8 and 9, Plate VI), especially the lower part of it, the fleshy portion of the thighs, over the liver, and the large nerve trunks.

Joint movements, or flexion of the arms and legs, with resistance, are employed in general massage. The patient is made to draw the limb up while the assistant offers slight resistance to his doing so. The 
patient then extends the limb while the assistant makes resistance in like manner. The movement is repeated several times, the number of times and the amount of resistance offered being regulated according to the strength of the patient. Figs. 32 and 33 illustrate arm flexion and extension.

An expert is required for the administration of massage in a thoroughly skilled manner. In another work * the author has given a full description of the various procedures and manipulations in massage.

37. Exercise. - Th is is of first importance as a general renovator of the tissues. The secretion of gastric juice is, under ordinary circumstances, proportionate to the amount of nourishment which the system is prepared to assimilate. Exercise creates a demand for food, and so stimulates both assimilation and secretion. best forms of exercise are those which will secure the most uni- Fig. 33.-Ara Extersion. form activity of the several parts of the muscular system. Riding, walking, rowing, and especially horseback riding are to be recommended as excellent. Gymnastic exercises and the judicious use of the "health lift" are also good; and for persons who, for lack of time or for any other cause, cannot adopt the other methods, these may be considered as almost indis-

* "The Art of Massage," Modern Medicine Publishing Co., Battle 'Creek. Wich. 
pensable. Such exercises as running, jumping, baseball playing, walking matches, and other violent exercises cannot be recommended. Trapeze exercise must also be discouraged on the same grounds. Agricultural. labor, especially the raising of small fruits and the cultivation of flowers, cannot be too highly recommended as forms of exercise for dyspeptic patients. For that large class of sallow-faced, weak-backed young ladies who have been made dyspeptics by idleness and too much coddling by fond mothers, the varied exercises of domestic labor are a most admirable panacea. And for the gaunt, hollow-cheeked, sunken-eyed young man, whose principal occupation is cultivating a moustache, smoking cigarettes, and swinging a slender cane, a lit- . tle wholesome experience in earning a subsistence by the sweat of his brow, instead of depending npon wealthy relatives, will prove a specific for the "softening" which begins in the brain, and extends to every part of the system.

Exercise before breakfast, while excellent for some, may be very harmful for others. Persons who complain of a feeling of " goneness," " f faintness," " sinking," and allied pains when the stomach is empty, and especially in the morning, must aroid exercise to any considerable extent before eating. Disregard of this rule often occasions loss of appetite and weakening of the digestion. Persons who are very weak must also avoid exercise before eating in the morning.

As before remarked, only gentle exercise can be taken soon after eating, or immediately before, without injury. Persons who feel a constant " sinking" or weakness in the stomach and bowels, will derive benefit, 
from wearing about the body a broad band of flannel. In many of these cases the Natural Abdominal Supporter will be found of great advantage. It is in these cases especially that complaint is made that walking produces coldness in the extremities, instead of serving to warm them.

One of the most marked effects of exercise upon digestion is to increase the activity of the gastric glands, and thus promote the secretion of the gastric juice. This has been clearly shown by recent experiments. It is hence apparent that in hyperpepsia little or no exercise should be taken immediately after eating, whereas in hypopepsia moderate exercise after eating may prove beneficial by increasing the activity of the gastric glands.

Exercises to Develop the Abdominal Muscles.-Development of the abdominal muscles is one of the most important of all curative measures in chronic indigestion, since its most obstinate forms are due to prolapse of the stomach, and those forms of constipation least benefited by a regulation of the diet or other curative means are usually the result of prolapse or dilatation of the colon. Development of the abdominal muscles benefits cases of this kind by holding the prolapsed organs in place, and also by increasing the pressure within the abdominal cavity, as well as the expulsive power. The following are among the most excellent exercises for developing the abdominal muscles:-

(1) Lying upon the back, raise the head forward as far as possible without the use of the arms. Repeat every five seconds, breathing in as the head goes back, and breathing out as the head is raised forward. 
(2) Raise the right leg as high as possible, and return to position. Repeat with the left leg, then with both legs. Empty the lungs as the leg is raised, filling the lungs as the leg is lowered to position. Repeat each exercise three to twenty times.

(3) Combine exercises 1 and 2, following the same instructions in regard to breathing (Fig. 10, Plate VI).

(t) Lying upon the back, with the legs extended, rise to the sitting position withont using the arms. Slowly return to the horizontal position; repeat three to twenty times. Exhale while rising to the sitting

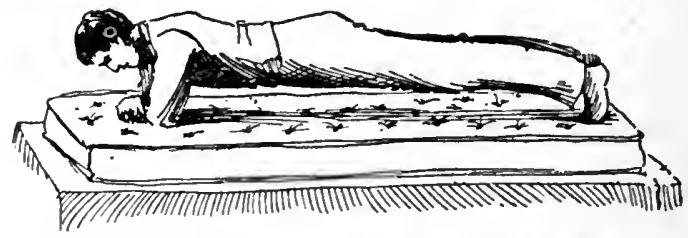

Fig. 34.- Exercise to Strexgthen Trexk Mcscles.

position; take one full breath after each movement.

(5) Repeat exercise 4, with the hands placed at the top of the head, and the elbows extended sideways and in line with the body.

(b) Lying upon the face, place the trunk, elbows, and toes in such a position that the whole body will be suspended upon the elbows and toes. Lower the center of the body until it rests upon the supporting surface; then raise to a horizontal position, repeating three to twenty times (Fig. 34).

(i) Lying upon the back with the knees drawn up, separate the knees as widely as possible, then bring them together again. The assistant should meanwhile make resistance to each movement by pressing first 
on the outer, then the inner surface of the knees (Fig. 11, Plate VI.)

(8) An excellent exercise for strengthening the abdominal muscles is the following: Standing with the feet separated as in Fig. 39 (page 319), and the hands placed apon the hips, the patient bends gently forward until a strain is felt upon the abdominal muscles, then rises to position, repeating the exercise a number of times.

38. Breathing Exercises. - The following exercises in breathing will be found very helpful to digestion. Before engaging in them it is well to take a little vigorous general exercise, as jumping up and down or a short run, which will create a thirst for air.

(1) Breathe in the ordinary manner for one minute, taking care that the greatest expansion is at the sides of the waist.

(2) Fill the lungs as full as possible, breathing slowly in and out, forcing the sides of the chest out as far as possible. Be careful not to confine the breathing to the upper part of the chest. Occupy equal time in breathing in and out, - about five seconds each for inspiration and expiration (Fig. 12, Plate VI).

(3) Take a deep breath occupying about two sec'onds, and breathe out in puffs, occupying about five or six seconds in expiration.

(4) Take a quick, deep inspiration, expanding the lungs as fully as possible, and follow with a prolonged expiration, sounding the syllable "ah."

(5) Standing or sitting, repeat each of the above exercises, at the same time raising the arms from the sides to a horizontal position while inhaling, and allow- 
ing them to return to position by the sides while exhaling.

(6) Repeat exercises 1 to 4, raising the arms from the sides to a vertical position, reaching overhead as

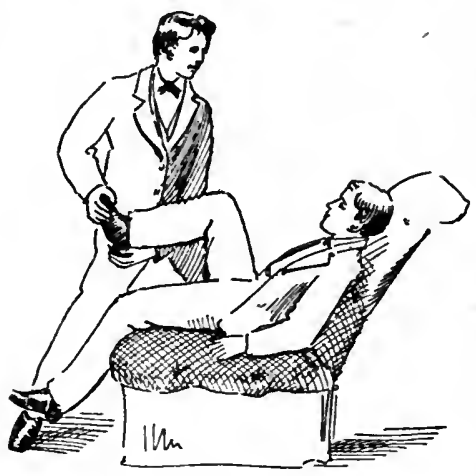

Fig. 35.- Lyixg, Kree Bexdisg.

high as possible at the end of inspiration, and allowing the arms to return to the sides during expiration.

(7) Chest Lifting.In Fig. 36 is shown a most excellent exercise, which, however, requires the aid of an assistant. The patient sits in a chair while the as sistant stands behind him. The assistant bends forward, grasps the arms a few inches below the shoulders, and lifts them upward and backward, the patient drawing in his breath at the same time. The arms should be drawn upward far enough to produce a decided strain upon the chest walls. After being held in position a few seconds, the arms are returned to the sides. The movements are repeated ten to twelve times a minute, the patient taking a deep breath after each movement. The clothing should be loose enough to allow the organs of respiration free play.

Manual Swedish Movements. - Among the most important of all forms of exercise useful in indigestion, must be mentioned Swedish medical gymnastics, or what are ordinarily termed "manual Swedish morements." Full directions for the employment of 
manual Swedish movements in disease will be found in a most excellent work no "Swedish Movements, or Medical Gymnastics," by Professor T. J. Harteiius, M. D., director of the Central Gymnastic Institute of Stockholm, Sweden, recently translated by Dr. A. B. Olsen, and edited by the writer; published by the Modern Medicine Publishing Co., Battle Creek, Mich.

The following groups of movements have been found especially valuable in the treatment of cases of chronic indigestion :-

39. For General Debility and Anemia.-

Repeat each of the following movements four to eight times:-

- 1. Lying, full breathing (38) (Fig. 12, Plate VI).

2. L y ing, knees drawn up, knees separating and closing with resistance (Fig. 11, Plate VI) $(37[7])$.

3. Replacement of the abdominal organs (Fig. 6, Plate VI).

4. Alternate head raising and leg raising forward (Fig. 10, Plate VI).

5. Sitting, a r m flexion, extension and rolling (Figs. 32, 33) (36).

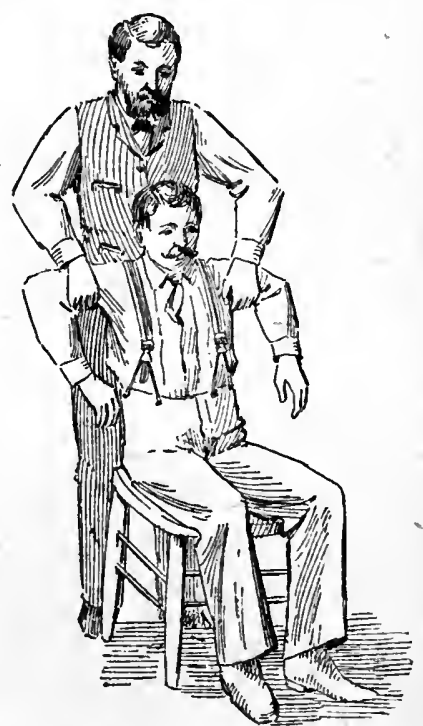

Fig. 36.-Chest Lifting.

6. Lying, inspiratory lifting (Fig. $\tau$, Plate VI), 
B.

Repeat each of the following movements four to eight times :-

1. Inspiratory lifting (Fig. 7, Plate VI).

2. Lying, knee bending and stretching (Fig. 35).

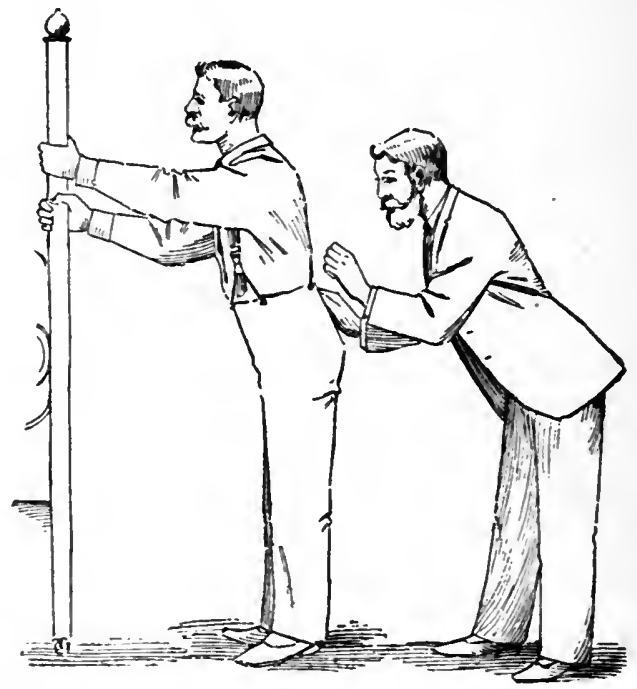

Fig. 37.-SACri:M Beatixg.

3. Hacking over liver, stomach, and spleen (Fig. 8, Plate VI).

4. Sitting, arms extended sideways, arms rolling.

5. Lying, raise trunk to sitting position $(37[t])$.

6. Lying, knee bending and stretching.

7. Replacement of abdominal organs (Fig. 6, Plate VI).

8. Sitting, chest lifting (Fig. 36) (38 [7]), 
40. For Dilatation of the Stomach. - The following movements may also be employed for dilatation of the stomach and prolapse of the stomach and bowels, which are usually present in cases of nervous headache :-

Repeat each of the following movements four to eight times :-

1. Chest lifting (Fig. 36).

2. Lying, knees bent, knees separating and closing (Fig. 11, Plate VI) (37 [7]).

3. Sitting, arm raising, full breathing (38 [5]). bending.

4. Standing, hands upon the hips, backward

5. Lying, knee bending and stretching (Fig. 35).

6. Lying, leg and head raising in alternation (Fig. 10, Plate VI) $(37[1,2,3])$.

7. Sitting, neck twisting and bending.

8. Inspiratory lifting (Fig. 7, Plate VI).

9. Reach-support-standing, sacrum beating (Fig. 9, Plate VI ; Fig. 37).

10. Sitting, gentle hacking of head (Fig. 8, Plate VI), stroking head and neck downward.

41. For Prolapse of Stomach, Bowels, Colon, Kidneys, and Other Abdominal Or. gans.-

Repeat each of the following movements four to eight times :-

1. Chest lifting (Fig. 36).

2. Replacement of abdominal organs (Fig. 6, Plate VI).

3. Lying on back, leg and head raising (Fig. 10, Plate VI) $(37[1,2,3])$. 
4. Inspiratory lifting (Fig. 7, Plate VI).

5 . Lying, knees bent, kneading of abdominal muscles.

6. Sitting, knees separated, trunk twisting right and left.

7. Lying, raise to sitting position (Fig. 3 ) $(37[4])$.

8. Sitting, bent forward, raise backward with pressure upon the loins.

9. Repeat exercise 2 with inspiratory lifting.

10. Repeat exercise 3.

42. For Dyspepsia with Dilatation of the Stomach. -

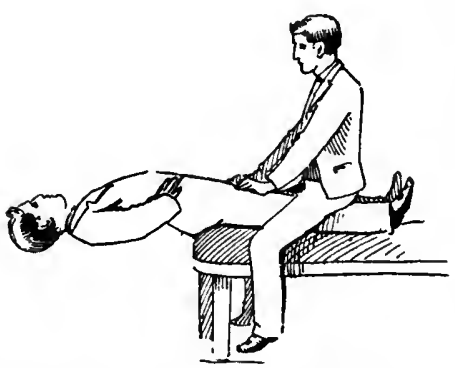

Fig. 38.- ExERcise For A bDomiNat Muscles.

Repeat each of the following movements four to eight times :-

1. Chest lifting (Fig. $36)$.

2. Reach-s u p portstanding, spine hacking and sacrum beating (Figs. 8, 9, Plate VI; Fig. 37).

3. Lying, knees bent, kneading the stomach

(Fig.5, Plate VI ; Fig. 35).

4. Lying, leg and head raising (Fig. 10, Plate VI) $(37[1,2,3])$.

5. Arm raising, full breathing (38 [5]).

6. Lying, knees bent, pressing under right and left ribs with vibratory movements.

7. Sitting, trunk twisting to right and left.

8. Repeat exercise 3. 
9. Lying, knees bent, knees separating and closing with resistance (Fig. 11, Plate VI) (37 [7]).

10. Repeat exercise 6.

11. Standing, forward bent, sacrum beating (Fig. $37)$.

\section{For Constipation. -}

Repeat each of the following movements four to eight times :-

1. Standing, feet separated, sideways bending to right and left (Fig. 39).

2. Chest lifting (Fig. 36) (38 [7]).

3. Sitting, twisting to right and left.

4. Inspiratory lifting (Fig. 7, Plate VI).

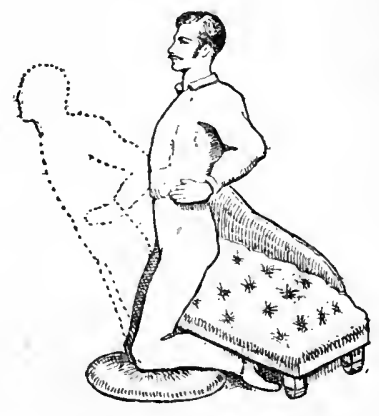

Fig. 39.-Staxding, SideWaYs BendiNg.

5. Lying, knees bent, kneading of the colon (Fig. 4, Plate VI).

6. Head and leg forward raising (Fig. 10, Plate VI) $(37[1,2,3])$.

7. Sitting, feet separated, describe a large circle with the head and shoulders, holding the trunk straight and the hips stationary.

8. Repeat exercise 4.

9. Reach-support-standing, sacrum beating (Fig. $37)$.

10. Sitting, arms stretched above head, bend arms with resistance, back supported by the knee of the attendant. 


\section{The Dry Abdominal Bandage.-A dry} flannel bandage worn about the abdomen is often of great value in cases of indigestion. The whole abdomen, from the lower end of the sternum to the pubes, should be covered by the bandage, which should fit the skin snugly. It should always be worn during the daytime by persons who wear the wet girdle at night.

45. The Abdominal Supporter. - The abdominal supporter (Fig. 40) consists, first, of two hard rubber pieces connected by an elastic webbing, which

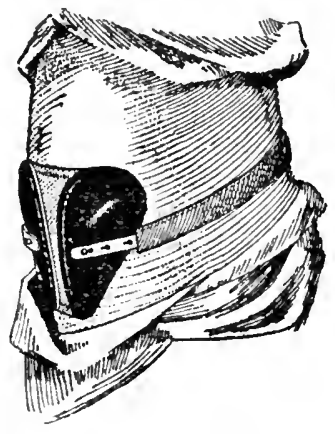

Frg. 40.- Nateral ABDOMLAL SUPPORTER. rest against the lower abdomen, being carefully shaped so as to make a uniform pressure; and second, a set of steel springs attached to a back piece, and so adjusted as to make pressure upon the hard rubber plates simultaneously backward and upward. The action of the supporter is almost a perfect imitation of the hands in lifting the prolapsed abdominal contents. After trying every form of supporter offered in the market, I have found the Natural Abdominal Supporter more satisfactory than any other, and have employed it in a very large number of cases. It is manufactured and sold by the Modern Medicine Company, Battle Creek, Mich.

46. Lavage. - The accompanying cut (Fig. 41) shows an approved form of stomach-tube, which is used in obtaining a test meal for the examination of the 
stomach fluid, and for washing out the stomach when necessary.

The most suitable time for washing the stomach is just before retiring at night, or five or six hours after the last meal. The operation is by no means so difficult or distressing as it might appear. The tube employed is of very soft, flexible rubber. It is not forced down into the stomach after the fashion of the old stomach-pump, but is simply swallowed as one would swallow a portion of food or a capsule. One end of the tube being introduced into the mouth and passed well back into the throat, the patient makes movements of swallowing whereby the tube is readily carried into the stomach. Water of about the temperature of the body is then poured in, either with or without the ad-

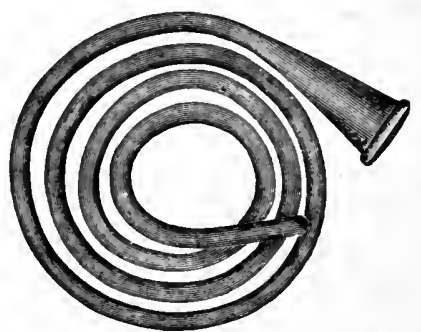

Fig. 41.-Stomach-Tube. dition of common salt and soda in the proportion of a teaspoonful of each to a quart of water. A powder consisting of three parts of bicarbonate of soda, one part of sodium sulphid, and one part of chlorid of sodium, may be advantageously used in cleansing the stomach, one teaspoonful of this powder being added to each pint of water ased in cleansing the stomach. After a pint or so of water has been poured into the stomach, the outer end of the tube is lowered, and by coughing or similar movements the contents of the stomach are forced through the tube into some proper receptacle. Repeat until the water is clear. 
Hot and Cold Lavage of the Stomach. - The purpose of hot and cold lavage of the stomach is to stimulate the secretion of pepsin and hydrochloric acid in cases of hypopepsia and apepsia. The general method is the same as that for lavage. The temperature of the fluid used may vary from $70^{\circ}$ to $120^{\circ} \mathrm{F}$., and the quantity from half a pint to a pint. Ordinarily, at the beginning, the extremes of temperature should not be more than $20^{\circ} \mathrm{F}$, or $85^{\circ}$ to $105^{\circ} \mathrm{F}$. Alternate hot and cold applications are a powerful means of stimulating vital activity, and the method is as serviceable when applied to nucous membranes as to the skin.

47. Charcoal.-Charcoal is raluable by reason of its remarkable absorbent qualities, whereby it takes up the poisonous substances produced in the stomach, thus preventing their absorption. It also exercises a very remarkable influence in preventing certain forms of fermentation in the stomach. It should be impalpably fine, so that it will not produce irritation of the mucous membrane, and should be freshly prepared. The author has succeeded in preparing, and has for many years employed, a special form of charcoal made from cereals, which he has found to be very much superior to the willow charcoal obtainable at the drug-stores. Charcoal must be taken in large doses (a heaping teaspoonful at least) to be of any service. It is best taken by mixing with a little water. At first use just enough water to moisten, then add more.

48. Charcoal Tablets.-The Modern Medicine Company, Battle Creek, Mich., U. S. A., have succeeded in preparing charcoal in the form of tablets, which are more convenient for use than the pulverized 
charcoal, as they can be handled readily, and taken with out water and without inconvenience, besides retaining their active properties indefinitely.

49. Antiseptic Charcoal Tablets. - These tablets are also prepared by the Modern Medicine Company, and are found to be the most effective means of preventing fermentation and decomposition in the stomach. They are especially valuable in acid and bilious dyspepsia, and in cases in which the tongue is heavily coated.

50. Lactic Acid.- Lactic acid, in the proportion of one ounce of the acid to three of water, is a useful remedy in cases of hypopepsia without fermentation. The dose should be one teaspoonful in water immediately after each meal.

51. Hydrochloric Acid.-Hydrochloric acid, in the proportion of one dram of the acid to three ounces of water, is useful in cases of hypopepsia and apepsia, but should not be used habitually. Two teaspoonfuls in water, half an hour after each meal, constitutes a dose ; a second dose may be taken half an hour later.

52. Laxatives. - Such fruits as tamarinds, figs, manna, and prunes, with a tablespoonful of bran mixed with boiling water, are all food laxatives of value in many cases. Antiseptic charcoal tablets (49), three or four after each meal, also have an excellent laxative effect. The advantage of these tablets is that their continued use does not destroy their efficiency, as is the case with most laxatives.

53. Cascara Sagrada. - The fluid extract of cascara sagrada is one of the most valuable of all drugs for temporary use as a laxative in cases of inac- 
tivity of the bowels. The dose is fifteen to thirty drops of the fluid extract taken at night in a little water and before breakfast, for a few days. It should, however, be used only for a short time, as it usually loses its effect when long continued.

54. Aqua Salina. - This is composed of sulphate of soda, one and one half ounces; sulphate of magnesia, one half dram; sodium chlorid, one fourth dram ; water, one pint. One fourth to one half a glassful, diluted with cold water, should be taken before breakfast. This is an excellent substitute for the best of the laxative mineral waters, bnt should be used only in case of emergency, when the enema cannot be employed, and should not be long continned.

55. Seltzer. - This is composed of sulphate of magnesia, one ouncè; tartaric acid, three and one half ounces; bicarbonate of soda, four ounces. The dose is two to four heaping teaspoonfuls, in water, before breakfast. Use same as $\mathbf{5 4}$.

56. Sulphur. - Sulphur is an old-fashioned but useful remedy as a laxative and intestinal antiseptic. It should be taken in ten-grain doses three times a day, or in doses of one half a dram at bedtime. - It may be mixed with a little molasses or brown sugar.

57. Antiseptic Dentifrice. - This excellent preparation for cleansing the teeth is based upon the extract of the bark of the famous soap-tree of South America and aromatic antiseptics. It may be obtained of the Modern Medicine Company, Battle Creek, Mich.

58. Cinnamon Solution.- - Add two teaspoonfuls of oil of cinnamon to a pint of water ; shake thoroughly. This is to be used as a lotion for rinsing and washing the mouth. 
59. The Perfection Vaporizer. - This is an excellent instrument for the vaporization and nebulization of medicated solutions for use in the treatment of affections of the throat and nose (Fig. 42). It is for sale by the Modern Medicine Company.

60. Benzoin Solution. - G $\mathrm{n} \mathrm{m}$ benzoin and all other balsams and essential oils are antiseptics,

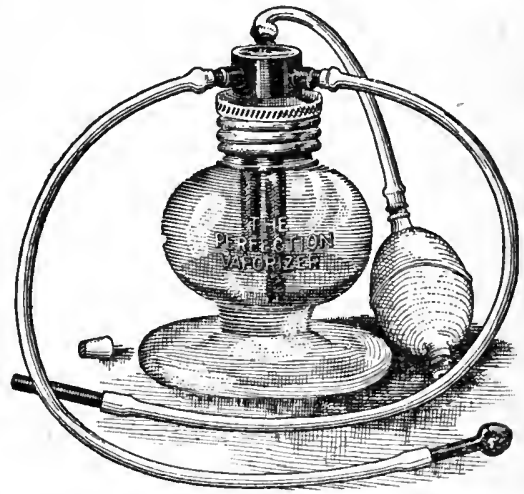

Fig. 42.-Perfection Vaporizer. and, used by means of the Perfection Vaporizer, are of great value in the treatment of diseases of the nose and throat. The following solution, in which benzoin is one of the principal ingredients, has been found especially valuable:-

R Compound Tr. Benzoin... drms. 6 Oil Cinnamon, Oil Win.

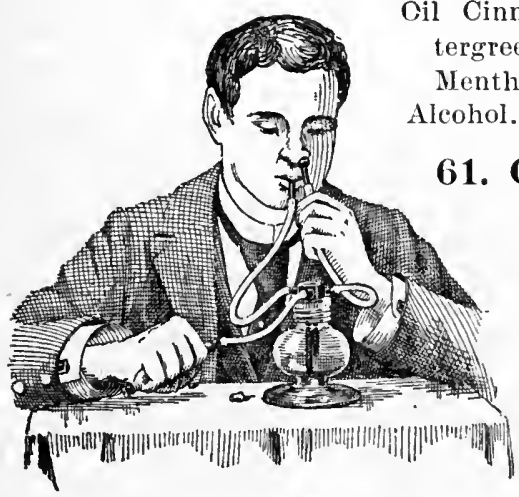

Fig. 43.- Vaporizer in Use.

B Menthol Crystals, Oil Eucalyptus, Tr.

Guaiac....aa.drm. 1 Alcohol...... dirms. 9

62. Subcarbon. ate of Bismuth. Subcarbonate and subnitrate of bismuth are 
two very useful drugs, which may be frequently used to adrantage in cases of indigestion accompanied by great irritation of the mucous membrane, and are invaluable in ulcer of the stomach and in hyperpepsia. The dose is fifteen to thirty grains before each meal. When lavage of the stomach gives pain, it should be followed by a dose of twenty to thirty grains of subnitrate or subcarbonate of bismuth.

63. Subgallate of Bismuth. - Subgallate of bismuth is an excellent intestinal antiseptic, five to ten grains to be taken before each meal. It is especially valuable in cases in which there is a tendency to looseness of the bowels, as it is somewhat astringent in its nature. It has a tendency to produce constipation when its use is long continued. It is, on this account, inferior to antiseptic charcoal tablets (49) as an intestinal antiseptic, but is especially valuable in cases in which an intestinal antiseptic is required in connection with an irritable condition of the stomach and in cases of intestinal catarrh.

64. Bicarbonate of Soda.- - This much-abused drug may be usefully employed in hyperpepsia and ulcer of the stomach, in which it should be taken in twenty- to thirty-grain doses, just before eating, in very severe cases, or two or three hours after eating in cases of less severity.

\section{Local Applications of Faradic Elec.} tricity. - Care should be taken that the sponges are moist, the conducting cord in good repair, and the battery in good order. Strong currents are generally best for relieving pain. When it is desired to exercise the muscles, the sponges should be applied near the 
edges of the muscle, and the current should be rapidly interrupted by removing the sponge and replacing it, so as to produce repeated muscular contractions. The application of electricity to the rectum is a most excellent means of relieving constipation. A special electrode is required for the rectum, a large, flat sponge being placed over the abdomen. In the absence of a special electrode, a small sponge may be applied to the anus.

66. General Applications of Faradic Elec. tricity. - As a general tonic, the current is applied in the following manner: A large, flat sponge is placed at the feet or connected with the feet by means of a foot bath, one sponge being dropped into the bath while the other sponge is passed over the whole surface of the body, - first the spine, then the arms and legs, then the trunk, - the strength of the current being sufficient to create a slight tingling sensation as the sponge is brushed over the surface, without producing muscular contraction.

For muscular effects, the following mode of application is employed : Two sponges, held a few inches apart, are applied to the muscles of the arms, legs, and trunk in such a way as to cause strong contraction of the muscles. By frequent interruption of the current by the removal and replacement of one of the sponges, repeated contractions are produced. Thus all the muscies of the body may be exercised.

67. The High Tension Sinusoidal Current. - This current is obtained from a specially constructed electrical apparatus (see Fig. 44), and is particularly efficacious in relieving pain, often succeed- 
ing when other means fail. The current is applied the same as the faradic eurrent $(65,66)$.

68. The Low Tension Sinusoidal Cur. rent. - This current is obtained from the same apparatus as the preeeding, but by a different adjustment of the machine. It is especially useful for exereise of the muscles, especially those of the trunk and abdomen. It is very valuable as a means of relieving chronic con-

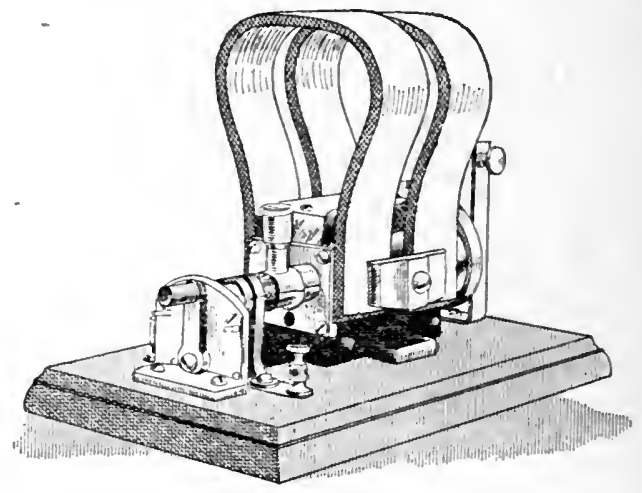

Fig. 4. Sinusoidal Electrical Apparates.

stipation. When used for this purpose, a metal electrode is placed in the rectum, and a large sponge over the abdomen. The current should be of sufficient strength to eause vigorous contraction of the abdominal muscles. The abdominal museles are thus strengthened and the nerves of the rectum stimulated.

69. Galvanization of the Spine and $A b$. domen. - A galvanic battery of thirty to fifty cells is required. A sponge about the width of the hand and ten or twelve inches in length is placed upon the central por- 
tion of the spine. Another sponge of the same length, and twice as wide, is applied over the abdomen opposite. As strong a current is passed as can be endured by the patient without severe pain or injury to the skin. The sponges should be well moistened, and should be closely adapted to the skin. Clay electrodes secure better contact than sponges.

70. Galvanization of the Sympathetic Nerve. - A galvanic battery is required as indicated in 69. A sponge the size of the hand is placed at the back of the neck, and another twice as large is placed over the umbilicus or the epigastrium. A current as strong as the patient can bear without injury to the skin is applied from ten to twenty minutes.

The Rest-Cure. - A course of rest-cure treatment is indicated in cases in which there is excessive nervous irritability, combined with nervous exhaustion, and especially in cases in which emaciation is a prominent symptom. Rest-cure should be followed by "General Nutritive Treatment," and afterward by "General Tonic Treatment," for which it is a preparation.

Fory A.

1. Absolute rest in bed, with exclusion of friends, is demanded. The patient should be kept quiet, and encouraged to exercise the mind as little as possible, to sleep all he can, and to rest both physically and mentally.

2. The patient should take four meals a day, - two major and two minor,- the major meals preferably at 8 A. м. and 3 р. м. ; the minor meals at 12 м. and 7 r. M., and should eat as much as can be digested. The major meals may consist of any of the articles 
included in Diet Lists Nos. 3, 9, and 10. The minor meals should be confined to the articles found in Diet Lists 5 and 6 , or such foods as are very quickly digested.

In cases in which the digestion is very much impaired, it is better to confine the diet chiefly to kumyzoon or buttermilk, and eggs. Bromose and granose (Diet List No. 25) are also especially useful in these cases. Bromose may be taken as a substitute for milk in cases in which milk does not agree well with the stomach. It is a most excellent food for the minor meals. When obtainable, granose should be used at the major meals as a means of regulating the bowels.

The patient should, on awakening each morning, have dry friction applied to the whole surface of the body by the hand, or, better, with a rough towel or a soft flesh-brush. At 10 А. .., hot and cold applications should be made to the spine (18), to be followed by a tepid sponge bath (1). Massage (36), at first very light, should be applied with increasing vigor from day to day. After the first week, the muscles may be exercised by flexion and extension of the arms and legs, the patient making resistance (see Figs. 32 and 33). The bowels should be moved daily by means of the enema (22), if necessary. The action of the stomach and bowels should be encouraged by massage of the stomach (31) and kneading of the bowels (30). Symptoms of indigestion should receive attention as eloewhere recommended.

\section{Form B.- Modified Rest-Cure.}

The same measures recommended in Form A should be employed, but the patient should be given a little 
exercise out of doors for an hour or two daily in a wheel-chair, or at first lying upon a cot. After a few days the patient may be allowed to walk a little, but should spend at least half the time each day in bed.

Form C.

The measures recommended for "General Nutritive Treatment," combined with rest in bed the greater part of the time, should be employed.

General Nutritive Treatment. - The purpose of nutritive treatment is to improve the patient's nutrition by increasing the demand for food, hence the appetite, and thus enlarging the store of food and blood. in the body without overtaxing the nervous system. Nutritive treatment is required in the case of very young and very old persons.

The following series of prescriptions are progressive. Feeble patients should begin with $\mathrm{A}$; while strong patients may begin with $\mathrm{B}$, or even $\mathrm{C}$. The patient should be promoted at the end of one, two, or three weeks, to the next prescription, and after completing this nutritive series, should begin the tonic series, page 333 .

A.

Fomentation over the stomach and liver every morning before rising, to be followed three times a week by a tepid sponge bath $\left(90^{\circ} \mathrm{F}\right.$.), rubbing with oil, and on other days by dry friction with a soft fleshbrush or a coarse towel.

General massage and massage of the stomach, bowels, and liver, about 10 A. м. daily. 
A short, warm full bath, with soap shampoo, followed by an oil rub, taken just before retiring at night, once a week.

Carriage riding, walking, bicycle riding, or other exercise in the open air suited to the patient's strength, from one to three hours daily.

Sun bath five to fifteen minutes daily, or whenever possible.

Swedish movements $(39$, A), daily.

Breathing exercises before and after each meal.

One or two hours' rest in a horizontal position before dinner.

\section{B.}

Warm full bath $\left(95^{\circ} \mathrm{F}\right.$.), with a cold pour over the spine and the liver twice a week. The bath should be cooled to $80^{\circ} \mathrm{F}$. for ten or fifteen seconds at the close, and followed with an oil rub. This bath is best taken at night just before retiring.

Hot and cold sponging of the spine for ten or fifteen minutes every morning, followed by a tepíd sponge bath $\left(85^{\circ}\right.$ F.) and an oil rub, with hacking over spine and liver.

Soap shampoo once a week.

Sun bath, out-of-door exercise, and massage as in A. Swedish movements $(39, \mathrm{~B})$.

C.

Short vapor, Turkish, or hot-air bath, or a wetsheet pack continuing twenty to thirty minutes, twice a week, followed by an oil rub at about 10 A. M. or at night just before retiring. 
Fomentations to the spine for fifteen minutes, followed by a cool $\left(75^{\circ} \mathrm{F}\right.$.) sponge bath and an oil rub every morning.

Swedish movements $(39, \mathrm{~B})$.

Exercise, sun baths, and massage as in $A$, with increased vigor.

General Tonic Treatment.-One of the essential features of tonic treatment is the application of cold water. Water is one of the best of all tonics. This treatment is indicated in cases of general debility, anemia, and most forms of ehronic disease. It is only contra-indicated in the cases of feeble, very young, and aged persons, and in those in whom there is a high degree of irritability or excitability. The treatment should be made progressive. The feeble patient should begin with $\mathrm{A}$, being promoted at the end of two or three weeks to $\mathrm{B}$, and later to $\mathrm{C}$. After completing the entire series, the patient may advantageously continue some of the measures reeommended,as a regular habit, particularly the cool morning sponge or shower bath, followed by vigorous rubbing, and the regular daily exercise out of doors.

A.

Hot sponging of the spine, a cool $\left(80^{\circ} \mathrm{F}\right.$.) towel or sponge bath every morning before rising, followed by hacking of the spine, spatting of the general surface, and oil rubbing.

At 10 A. M. general massage or Swedish movements.

General applications of electricity - faradic (66) or sinusoidal (67) - daily. 
Swedish movements $(39, \mathrm{~A})$.

Exercise ont of doors, carriage riding, horseback riding, bicycle riding, according to the patient's strength, daily from two to four hours.

B.

In addition to $A$, the patient should three times a week take fomentations over the stomach and liver, followed by a rubbing wet-sheet. This treatment may be given at bedtime, unless the patient is apt to be greatly fatigned at night. In such cases it should be taken earlier in the day, but not within two hours of a meal.

Swedish novements $(\mathbf{3 9}, \mathrm{B})$.

C.

The patient should take, each morning, a fomentation over the abdomen, hot and cold sponging of the spine, a cold $\left(\pi^{\circ} \mathrm{F}\right.$.) sponge bath, followed by an oil rub, liacking orer the spine and liver, and general massage.

At 10 A. M. or at bedtime, three times a week, a cold wet-sheet pack for twenty to thirty minutes, followed by a rubbing wet-sheet and an oil rub.

Swedish movements, general applications of electricity, massage, and out-of-door exercise as in $\mathbf{A}$ and $\mathrm{B}$.

Mechanical Swedish Movements. - For many years I have made use of mechanical means in the treatment of indigestion and constipation. $\mathrm{Pa}$ tients under my care have derived special benefit from the use of the vibrating chair, the kneading apparatus, 

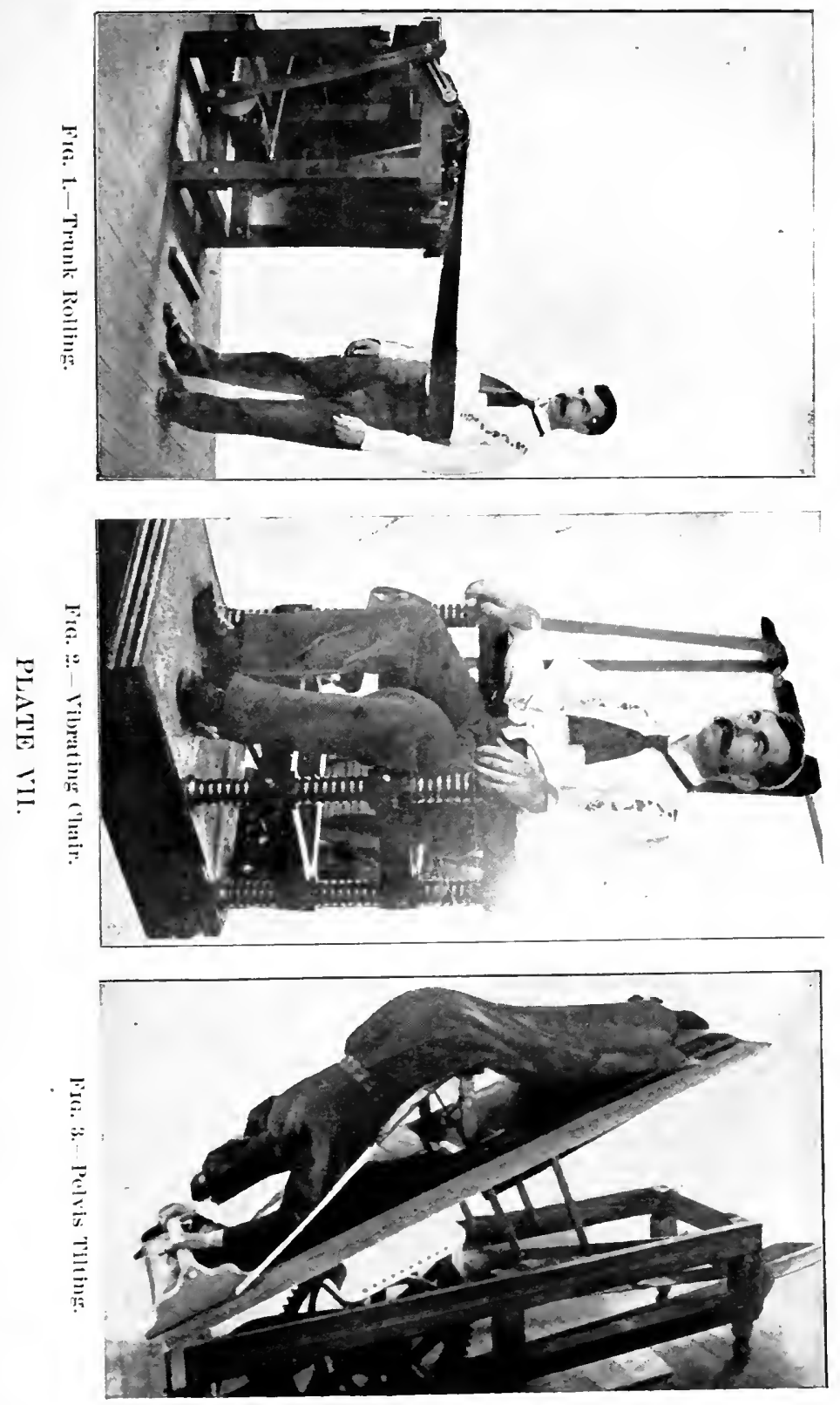


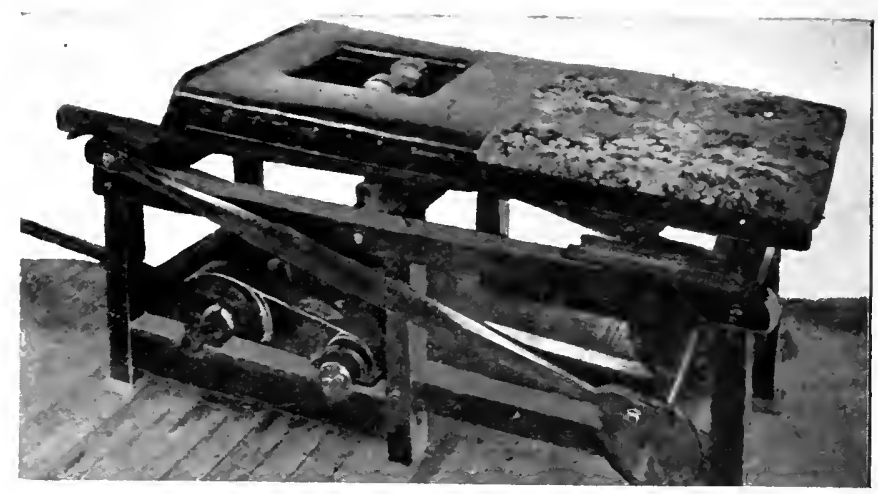

Fir. 1. - Ipparatus for lincading thr Abdomen.
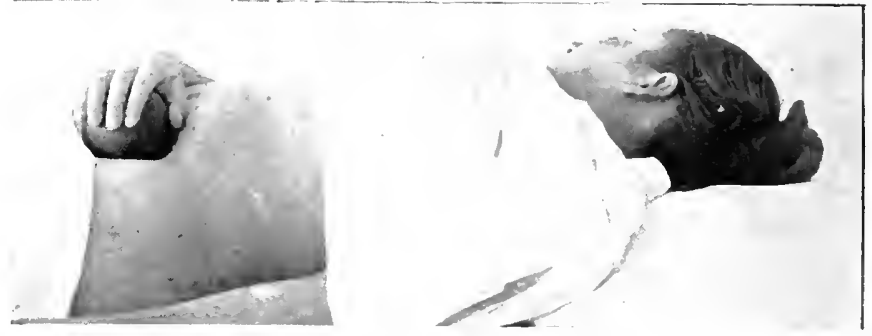

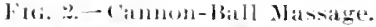

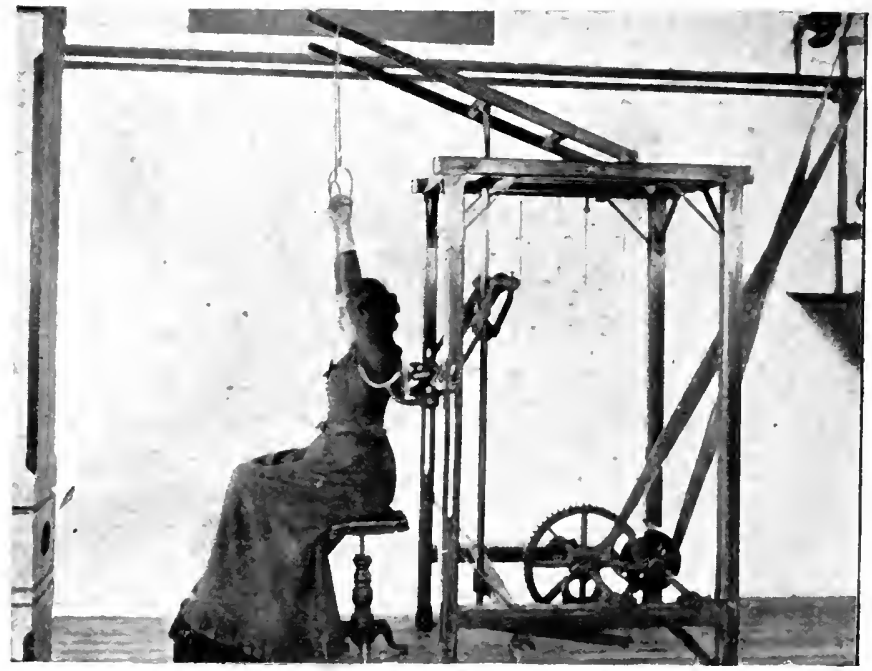

Fici, 3.- Mechanleal Respiration. 
and the trunk-shaking apparatis, which are illustrated in Plates. VII and VIII: The following description of these machines is copied from the author's work, "The Art of Massage," previously mentioned :-

"The Vibrating Chair.-Fig. 2, Plate VII, represents a vibrating chair devised by the author in 1883 , and in constant use since that time at the Battle Creek Sanitarium. The usual rate of vibration employed is twenty per second. A person needs to experience but a single application to become convinced of the powerful physiological effects which may be produced by mechanical vibration. When seated in the chair, strong vibratory movements are experienced, in which the whole body takes part. The greatest amount of force is applied to the lower portion of the trunk. The vibratory impulses communicated are felt-powerfully in the lower bowel, and have a decided stimulating effect upon the rectum.

"Mechanical Treading.-Mechanical kneading of the abdomen is one of the most useful of the several forms of kneading ; indeed, it may perhaps with justice be said to be the most useful of all. It is best administered by means of the apparatus shown in Fig. 1, Plate VIII. This apparatus, which the writer had constructed for the purpose several years ago (1883), and has had in constant use since, consists of a table with a large aperture near the center of the top. In this opening plays a series of six vertically placed bars, each surmounted by a suitable pad. "Each bar is separately actuated by a cam, or eccentric, so that it has its own independent motion. These six eccentrics are so arranged as to give a wave-like form to the combined 
movements of the six kneading pads. Simultaneously with the vertical movement of this kneading device, the table-top, with the patient which it bears, is made to move back and forth, thus changing the relation of the pads to the abdominal surface, and causing them to knead the entire abdomen. The two sets of movements are so timed that the wave-like kneading movement is made to follow very closely the course of the colon, thus bringing this part of the intestine especially under control.

"Trunk Rolling.- The apparatus represented in use in Fig. 1, Plate VII, consists of a pair of pulleys moving in alternation and in opposite directions, a fraction of a revolution in each direction. To each pulley is attached one end of a broad strap, which is passed around the trunk in such a manner that, as the strap is pulled first in one direction and then in the opposite, the tissues are acted upon very much as in certain forms of palmar kneading. When applied about the waist, it is a very excellent means of administering a rolling movement to the muscles of the trunk, and a shaking movement to the viscera ; when applied to the shoulders, the effect is that of deep kneading. This apparatus, devised by the author about ten years ago, is a favorite with patients who are under treatment by mechanical massage. Its application is so vigorous that it is not continued longer than from two to four minutes.

"Pelvis Tilting. - The purpose of this derice (Fig. 3 , Plate VII) is to aid in restoring the prolapsed organs to a normal position. The apparatus consists of a tilting table which moves rhythmically from a horizontal 
position to that shown in the cut, in which the position of the patient is such as to cause the abdominal organs to be dragged upward by gravity. It is very useful in relieving pelvic congestion and other symptoms commonly present in cases in which the pelvic and abdominal organs are prólapsed.

"Mechanical Respiration. - In Fig. 3, Plate VIII, is shown an apparatus by means of which artificial respiration may be mechanically administered. In its use the patient is seated upon a stool, the arms being placed over movable rests, which fall in the axillæ. The back is supported by a padded rest placed between the shoulders: When the macline is set in motion, the shoulders are lifted upward and backward in such a way as to expand the chest in an efficient manner, producing a strong inspiratory movement quite independent of any effort on the part of the patient. The effect is to correct the condition known as flat or hollow chest, and to give flexibility to the chest walls when they have become rigid in consequence of insufficient use. This apparatus is in part modeled after a similar arrangement by Zander, but several improvements have been added; among others being a device by means of which the arms, as well as the shoulders, are raised, thus increasing the vigor of the inspiratory movement. "Cannon-Ball Massage.-A cannon-ball covered with leather is a valuable mechanical accessory in the application of abdominal massage. A ball weighing from four to sfx pounds is usually employed. The ball is simply rolled upon the abdomen, following the course of the colon from right to left. I have found the cannon-ball very useful when employed in connec- 
tion with other measures of treatment. It has this advantage, that it may be employed by the patient himself. It should be used for fifteen minutes morning and evening. In the morning it may be employed just before rising, or half an hour after breakfast."

Water-Drinking. - In cases of obstinate constipation, due to inactivity of the liver, water-drinking is of advantage, if the stomach will bear it. The quantity of water which can be taken will vary from a single glassful before breakfast to six glassfuls a day in the interrals between meals. Repeated experiments by the most eminent physiologists have shown that the liberal use of water as a bererage is a great promoter of vital activity, not only of the liver, but of other vital organs. This measure must not be carried to excess, however; it should be discontinued if it disturbs digestion.

Water-drinking has been rery much abused within the last twelve or fifteen years, since it was brought proninently before the public by Dr. Salisbury and Dr. Cutter, of New York. In cases of dilatation of the stomach, which is very common among dyspeptics, constituting nearly one half the entire number, copious water-drinking is often productive of great mischief. The dilated stomach being unable to absorb fluids rapidly, and retaining for a long time the large quantity of water introduced, becomes abnormally distended and overweighted, and the patient's condition is thus aggravated rather than benefited. The use of the stomach-tube in washing out the stomach largely obviates the necessity for copious water-drinking. In cases in which water-drinking must be interdicted on account 
of dilatation of the stomach, a sufficient amount of moisture may be supplied by slowly introducing a quart or two of water into the colon daily, preferably at night, allowing as much of the liquid to be absorbed as possible.

- The Hunger-Cure.-Fifty years ago the hungercure was much practised by the followers of Dr. Joel Shew, and at the primitive water-cures which were at that time to be found in various parts of the Middle and Eastern States. The advocates of the hunger-cure extolled it so highly, however, and used it so indiscriminately, that it fell into disrepute, and fasting is now seldom mentioned as a mode of treatment. However, the writer is of the opinion that abstinence from food is one of the most important and valuable of curative measures in a great variety of morbid conditions, and especially in certain forms of indigestion.

The fasting exploits of Drs. Tanner and Griscom, who abstained from food for forty and forty-two days respectively, and of the Italian faster who, in London, under conditions of strict surveillance, abstained wholly from food for the full period of sixty days, have dissipated the erroneous notions formerly held respecting the immediate necessity of food as a condition of life. The body contains within itself a considerable store of nutrient material, upon which it is able to draw in case of need.

In all conditions in which the system is laboring under the influence of poisons absorbed from the alimentary canal, abstinence from food is the surest and sometimes an indispensable means of prompt relief. These intestinal poisons are due to the action of germs, 
and result from the decnmposition of food substances consequent upon the growth and development of microbes. Germs, like other living organisms, require food for growth. The quickest method of getting rid of them and their evil effects, is to starve them out by abstinence.

The advantage of withholding food in fever, acute rheumatism, and inflammation of the stomach and bowels, has long been recognized. Fasting is equally useful in many cases of biliousness, bilious dyspepsia, and dilatation of the stomach. Nothing will so quickly relieve an attack of sick-headache as abstinence from food. Fasts of two or three weeks' duration, as practised in water-cures half a century ago, are not, however, to be recommended; but the omission of a meal when one feels slightly indisposed, with loss of appetite, and perhaps slight headache, will often prevent a more serious illness, and quickly restore the vital equilibrium. A number of years ago the writer succeeded in effecting a cure in a case of very pronounced dilatation of the stomach, by requiring the patient to abstain from taking food by the mouth for two weeks. Nutrition was mantained by means of the nutritive enema. At the end of the two-weeks' fast the stomach was found to be reduced to nearly its normal dimensions.

I had under my care at one time a lady who many years before had submitted to the hunger-cure. This patient was suffering from obstinate constipation, the result of prolapse of the stomach and colon, and dilatation of the colon. She claimed that the two-weeks' fast which she had undergone some twenty years before, had resulted in a complete cure of the constipa- 
tion for more than twelve years. So severe a measure is not necessary, however, in cases of this kind. A proper regulation of the diet, the support of the prolapsed viscera by a suitable abdominal supporter (Fig. 40 , page 320 ), and the employment of such curative measures as electricity, manual Swedish movements, gymnastics, and hydrotherapy, will suffice to effect a cure, if employed intelligently and perseveringly.

Fasting methods can very easily be abused. Prolonged fasting should never be undertaken without the advice and careful supervision of a physician. I have, in my experience, met with very few instances in which I have thought it proper to advise a prolonged fast, but have in a great number of cases observed decided benefit as the result of omitting a few meals, or fasting for a day or two.

Great benefit may be received from what might be termed a partial fast, in which the patient subsists wholly upon some one simple article of food taken in moderate quantity. Fruit, as ripe apples, grapes, or peaches, is best suited for this purpose. The effect of such a regimen, which may be followed for a week without injury, providing the patient is resting, is to purify the general system in a wonderful manner, first, by preventing, through the destruction of microbes, the production of poisons in the alimentary canal, which overtax the liver in their destruction, and the kidneys in their elimination; and, second, by encouraging the activity of the kidneys in removing impurities from the blood. Fruit is a natural diuretic.

Healthful Dress.- As has already been mentioned, dress is a matter of no small consequence in 
the treatment of chronic dyspepsia. Any pressure upon the stomach is highly injurious. Men, as well as women, frequently suffer injury from constrict. of the waist, in supporting their clothing by means of a belt. The writer has met with two eases of floating kidney in men, in which the condition was evidently due to contraction of the waist. In one case a blacksmith had usually supported his clothing by means of his leather apron-strings tied tightly about his waist. In the other case an army officer had been injured in the same manner by the constriction of his sword-belt.

The clothing should fit the body so loosely that there will be ample room for full expansion of the chest at the waist; otherwise, with each inhalation the stomach is crowded down out of position. The clothing should be wholly suspended from the shoulder's, not merely by straps, but by waists ; or it is still better to wear what are known as "union garments," in which the upper and lower garments are united in one. Plates IX and $\mathrm{X}$ show some excellent designs for healthful dress for women which have been designed in the Dress Department of the Battle Creek Sanitarium, from which patterns may be obtained.

In addition to wearing the clothing loose enough to give every organ perfect freedom of action, it is of the greatest importance that the extremities be kept thoroughly warm. Cold hands and feet are very common with dyspeptics. It will generally be found best to wear flannel undergarments throughout the year, graduating the thickness to the temperature. In extreme cases of disturbed circulation, it may be peces: 


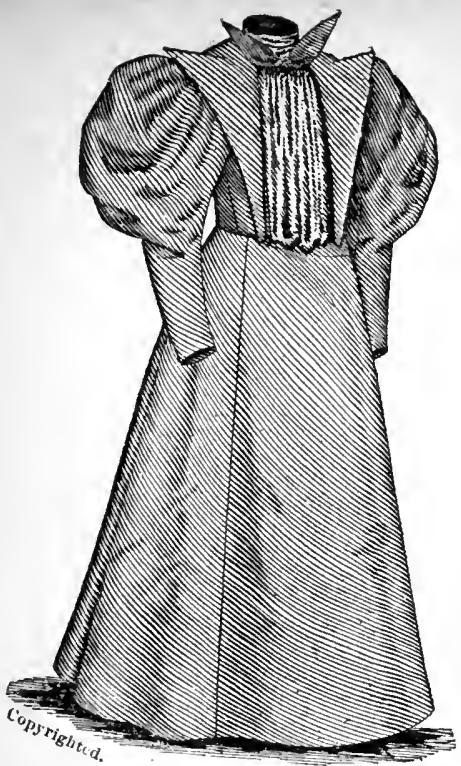

Fig. 1.- Variété Costume.

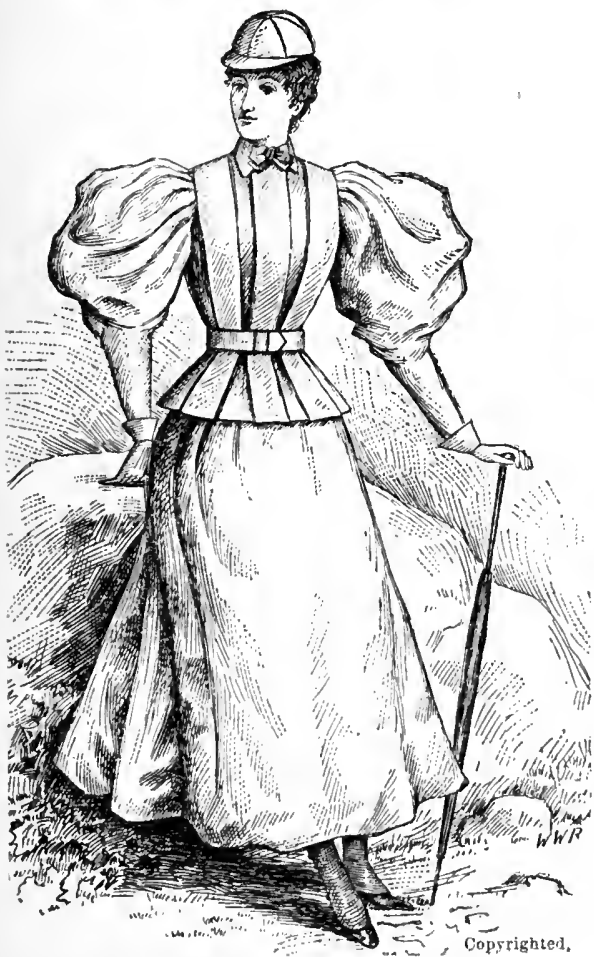

Fı. 3.- Norfolk Jacket with

Filrt of Business Sult.

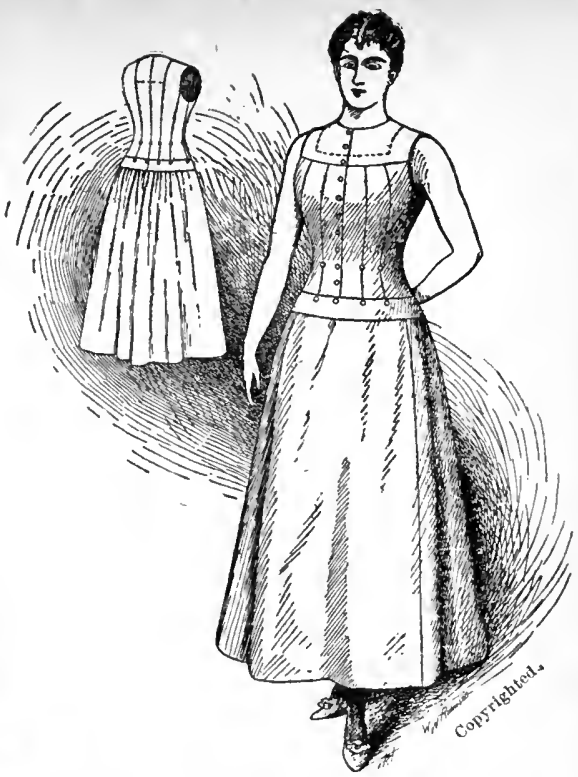

FIG. 2.-Freedom Waist with Skirt.

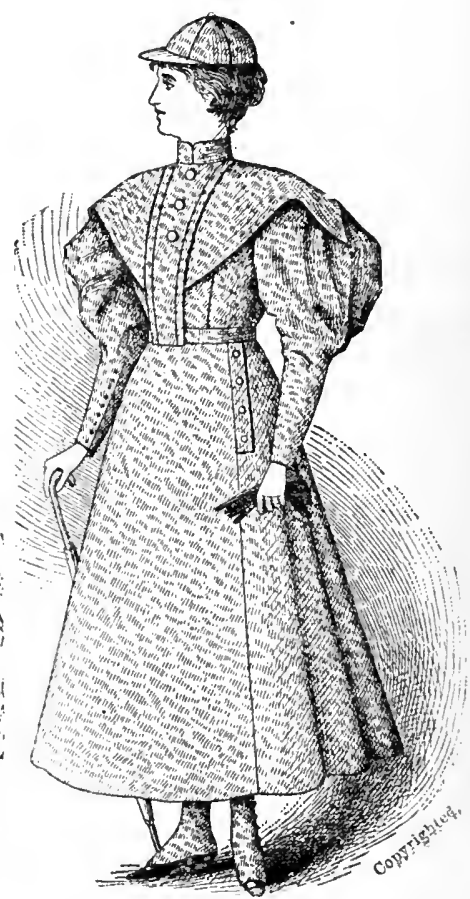

F1G. 4.-Woman's Practical Business Costume, 


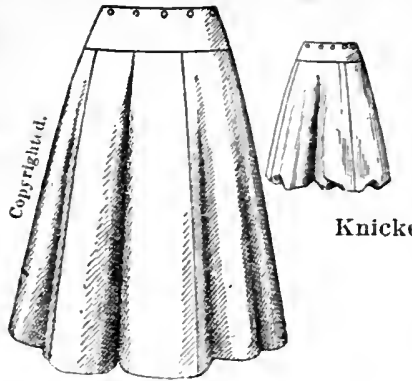

Fin, 5.-Divided Skirt with Circular Yoke-Front.

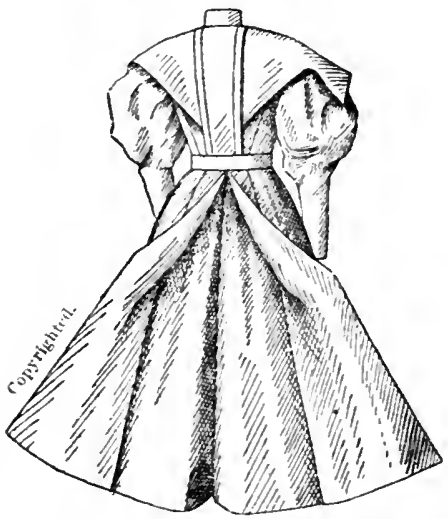

FIG. 7.-Fig. topened in Back to show Divide.

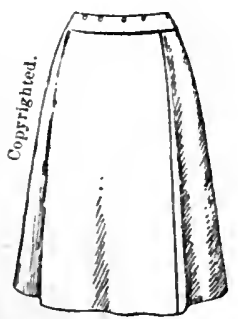

Fic. 10.-Shirt with Circuiar Yoke.

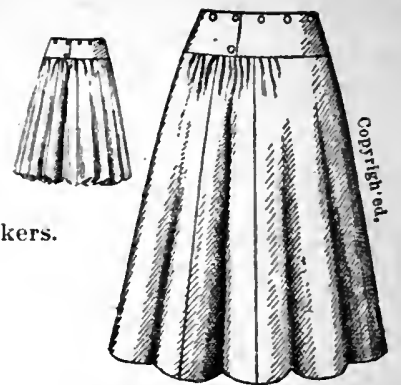

Fig. 6.-Divided Skirt witb Circular Yoke-Back.

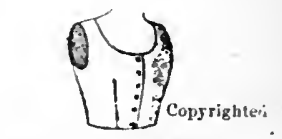

Fia. 8.-Skirt Waist

- Front.

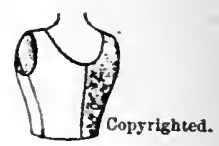

Fig. 9.-Skirt Taist

- Back.

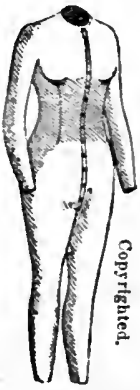

F1g. 11.-Union Suit-Front.

Fig. 12--Union Suit-Back.

PLATE $\mathrm{X}$. 
sary to change the clothing once or twice a day to accomplish this.

Sleeping. - It is of great importance that sufficient sleep be obtained by those suffering from indigestion, though sometimes this seems impossible on account of the nerrousness occasioned by this disease. It is generally best to retire early, but there is no virtue in getting up in the morning at an early hour unless the body is recuperated by rest. Sleep must be obtained, and on many accounts it is better to take it in the forepart of the night; but if not secured then, it should be taken at other times. Sleeplessness indnced by anxiety is often a cause of dyspepsia. It is a great obstacle-in the way of successful treatment.

It is an excellent practise to retire for rest for an hour or two before the mid-day meal. Many dyspeptics are able by this means to recruit sufficient energy to digest the second meal, while otherwise they suffer from indigestion after dinner, although capable of digesting breakfast without difficulty.

Traveling. - Many physicians are in the luabit of recommending patients upon whom they have exhausted their skill, to seek health by traveling. Thousands annually leave their homes, and at great expense visit various watering-places, mineral springs, etc., in this country and in Europe, in accordance with such advice. Some return much benefited; but the majority are no better except for the rest. This is due to the fact that traveling does not remove the real cause of the difficulty, and may often increase it. In general, it is next to impossible to secure, while traveling, either regularity of meals or other habits, or a 
proper quality of food. This, of course, to a great degree counteracts the benefit which might otherwise be derived.

The advantage of special climates is undoubtedly very greatly overrated, though a cool climate may generally be considered as best, especially for those suffering with bilious dyspepsia. With nervous dyspeptics, howerer, a warm climate seems to agree better, as it occasions less disturbance of the circulation.

Mental and Moral Treatment. - This is too important a part of a successful plan of treatment to be neglected. The gloomy despondency which so commonly goes with this disease, must be steadily combated by a determination to be cheerful. The disposition to. fret and worry, and to dwell upon the unpleasant or painful features of one's condition, must be fought against with firmness and resolution. The dyspeptic who allows his mind constantly to dwell upon his stomach, and who speculates upon the probabilities respecting the digestion of each morsel of food as he swallows it, will be certain to remain a dyspeptic. This unfortunate tendency on the part of sufferers from this disease is a great impediment to recovery. The mind should be diverted from self as much as possible at all times, and espeeially while eating. The habit many dyspeptics have of talking constantly about themseives, which sometimes amounts almost to a monomania, cannot be too strongly condemned. Too great solicitude about the stomach, the diet, etc., is worse than none at all, 


\section{QUACKS AND NOSTRUMS.}

THERE is no class of maladies the victims of which have been so constantly and extensively a prey to the quack and the patent-medicine vender, as disorder's of the stomach. The chronic dyspeptic, daily tormented by the discomforts of his malady, eagerly secks relief from any source, and is readily beguiled by the deceptive and alluring descriptions of newspaper advertisers, patent nedicine almanacs, and manufactured testimonials, to try first one and then another of the thousand and one nostrums which are displayed upon the druggist's shelves and catalogued upon country barns and fences. Quite a lárge proportion of dyspeptics thus become addicted to the use of patent medicines. The writer has met many persons who might very justly be termed " nostrum maniacs." They eagerly scan every newspaper that comes into their hands for the announcement of some new medicine of this kind, and as soon as possible obtain it for trial.

The great charm of the patent medicine is the secrecy thrown about it, and the idea often positively maintained - always encouraged - by the manufacturer, that his particular nostrum contains some newly discovered remedy, the virtues of which have been entirely unknown to scientific medicine. One of the best means of dissipating this fallacy is to expose the fact 
well known to the scientific physician, that all patent medicines and nostrums are either inert and worthless, because of their inefficiency or their injurious character, or else are simply cheap and well-known drugs with which erery physician is familiar, and which may be obtained from any druggist for a very small fraction of

- the price charged for the nostrum. As a rule, the first cost of the patent medicine is scarcely one twentieth the price charged for it. The manufacturer puts his ill-gotten gains into newspaper advertisements and huge signs painted upon board fences, barns, rocks, and conspicuous places along highways and railroads, instead of into the medicine bottle. For an exposé it is only necessary to publish the composition of these nostrums. Here are a few of them:-

Stoughton Bitters. - Orange peel, 6 oz.; gentian root, 8 oz.; Virginia snake root, $1 \frac{1}{2}$ oz. ; American saffron, $\frac{1}{2}$ oz.; red saunders, $\frac{1}{2}$ oz. ; alcohol, 4 pts.; water, 4 pts.

Brown's Iron Bitters. - Iron, 1 gr.; calisaya bark, 2 gr.; phosphorus, 1-200 gr.; coca, 1 gr.; viburnum prunifolium, $1 \mathrm{gr}$.

Hop Bitters. - Hops, 4 oz.; orange peel, 2 oz.; cardamom, $2 \mathrm{dr}$.; cinnamon, $1 \mathrm{dr}$.; cloves, $\frac{1}{2} \mathrm{dr}$.; alcohol, 8 oz.; sherry wine, 2 pts.; simple sirup, 1 pt.; water, sufficient.

Hostetter's Bitters. - These bitters contain, according to the Medical Bulletin, the following ingredients : Sugar, 2 lbs.; calamus root, 2 lbs.; orange peel; 2 lbs.; Peruvian bark, 2 lbs.; gentian root, 2 lbs.; columbo root, 2 lbs.; rhubarb, 8 oz.; cinnamon, 4 oz.; cloves, $2 \mathrm{oz}$; diluted alcohol, 4 gals. 
German Bitters.-German camomile, 2 oz.; sweet flag, 2 oz.; orris root, 4 oz.; coriander seed, $1 \frac{1}{2}$ oz.; centaury, $1 \mathrm{oz}$; orange peel, $3 \mathrm{oz}$; alcohol, 4 pts.; water, 4 pts.; sugar, 4 oz.

Stomach Bitters. - Gentian root, $1 \frac{1}{2}$ oz.; cinchona bark, $\frac{1}{2}$ oz. ; orange peel, $2 \frac{1}{2} \mathrm{oz}$; ; cinnamon cort., $\frac{1}{4} \mathrm{oz}$.; anise seed, $\frac{1}{2} \mathrm{oz}$; ; coriander seed, $\frac{1}{2} \mathrm{oz}$; cardamom seed, $\frac{1}{8}$ oz.; gum kino, $\frac{1}{4}$ oz. ; alcohol, 1 pt.; water, 4 qts.; sugar, $1 \mathrm{lb}$.

French Absinthe.-Oil wormwood, 1 dr.; oil melisa, 15 drops ; oil anise, $2 \frac{1}{2} \mathrm{dr}$.; oil star anise, $2 \frac{1}{2} \mathrm{dr}$.; oil fennel, $\frac{1}{2} \mathrm{dr}$.; oil coriander, 3 drops ; alcohol, 14 pts.; water, 6 pts.

Vinegar Bitters. - The following is Dr. Gibbon's account of the origin of Walker's Vinegar Bitters, a specimen of which the writer analyzed several years ago, and found to contain five per cent. of alcohol :

"This 'bitters' is one of the nastiest nostrums, and is introduced and largely sold by the most extensive and brazen advertising under the false pretense of being free from alcohol. It originated with the cook of a party which traveled overland as a mining company to California in 1849 ; he settled in Calaveras county, and having no success as a miner, turned his attention to the bitter qualities of the herbs growing about him, and came to San Francisco with the idea of making and vending a nostrum to be called 'Indian Vegetable Bitters.' He fell in with an enterprising druggist, who saw money in the project, and joined him. At the suggestion of the latter, the 'Indian' was struck out, and as the concoction got sour by fer- 
mentation, it was concluded to call it ' Vinegar Bitters,' and to identify it with the temperance movement. The native herbs, which became rather troublesome to collect, were discarded; and aloes, being a cheap bitter," was substituted. 'Nine sick people out of ten,' said the druggist, 'will be cured by purging,' therefore the aloes and Glauber's salt. So the cook turned doctor, the decoction became sour, and of Califormian instead of Indian paternity, and 'Dr. Walker's Vinegar Bitters' began its career in the newspapers and on the shelves of the drug-stores."

The statement has recently been made that "Vinegar Bitters" is now manufactured of sour beer and aloes.

Lee's Anti-Bilions Pills. - Calomel, 36 gr.; jalap; $60 \mathrm{gr}$.; gamboge, $12 \mathrm{gr}$; t tartar emetic, $3 \mathrm{gr}$.

Tropic Fruit Laxatice. - Jalap, powdered, 5 parts ; senna, powdered, 5 parts; sugar, 5 parts; tamarind pulp (E. I.), 30 parts.

Curter's Little Liver Pill.s.- Podophyllin, $1 \frac{1}{2}$ gr.; aloes (Socotrine), $3 \frac{1}{2}$ gr.; mucilage of acacia, sufficient.

Simon's Liver Regulator.-Hepatica, 1 oz.; leptandra, 1 oz.; serpentaria, 1 oz; ; senna, $1 \frac{1}{2}$ oz.

Radway's 'Regulating. Eills.-Eách box contains from twenty-nine to thirty-one sugar-coated pills of unequal size. They"consist of 30 grains of aloes, 15 grains of jalap, 8 grains of gamboge, and some inert substance.

Eno's Fruit Salt. - Soda bicarbonate, 168 parts ; tartaric acid, 150 parts; Rochelle salt, 110 parts.

Hamburg: Tea. - This is composed of senna leaves and stems, coriander frnits, manna, and tartariciacid. 
Garfield Tea.-This consists chiefly of senna leaves and couch grass.

Holloway's Pills.-Aloes, 2 dr.; rhubarb, 1 dr.; capsicum, 20 gr.; saffron, 5 gr.; sulphate of soda, $5 \mathrm{gr}$. Hamburg Drops. - Powdered Socotrine (aloes), $1 \frac{1}{2}$ oz.; American saffron, $\frac{1}{2}$ oz.; tincture of myrrh, $16 \mathrm{oz}$.

R. V. Pierce's Pleasant Purgative Pellets._Each little bottle contains from twenty-eight to thirty-six small sugar-coated pills of unequal size, and weighing, in all, from eighteen to-twenty-two grains. Their cathartic effect is due solely to podophyllin, the resin of the root of the May-apple.

Dr. Hall's So-called "Secret." - For several years, a man styling himself Dr. A. Wilford Hall, of New York City, has been advertising and vending about the country a pamphlet purporting to disclose a discovery made by himself something more than forty years ago. The so-called discovery of Dr. Hall's is this :

Having had dyspepsia for a number of years, and being greatly troubled with constipation, he resorted to the enema as a means of emptying his bowels, and discovered, as he asserts, that it was possible to inject a gallon of water into his colon by means of a bulb syringe.

This is the whole of Dr. Hall's so-called discovery. He recommends the enema as a substitute for nature's method of relieving the bowels, to be employed by all persons, sick or well, and claims that persons who will adopt this method of relieving the bowels, will be proof against most of the diseases to which human flesh is heir, mentioning particularly such disorders as Bright's 
disease of the kidneys, smallpox, and other grave and contagious maladies. This so-called discovery is embodied by Dr. Hall in a cheaply printed pamphlet costing about two cents, for which he charges the modest sum of four dollars.

Some three or four years ago, the writer, in the magazine of which he is editor, Good Health, exposed in a most thorough manner the fraud perpetrated by Dr. Hall in professing to be the discoverer of the enema as a mode of emptying the bowels. Whoever was the discoverer of this method of mechanically moving the bowels, it certainly was not Dr. Hall. Dr. Shew, a water-cure physician, advocated the method in this country some years before Dr. Hall professed . to have made the discovery, and Preissnitz had employed it long before. The enema may be properly used as a means of emptying the bowels when they are not emptied by the unaided efforts of nature, and is preferable to the habitual use of laxatives or cathartics of any sort; but the practise of daily introducing a large quantity of water into the alimentary canal, has the effect to destroy the normal sensibility of the bowels, and to establish an abnormal condition, so that this large amount of water becomes necessary as a stimulus to provoke an evacuation of the intestines.

It was long ago discorered by physicians that the habitual use of the enema as a means of stimulating the movement of the bowels, is by no means free from evil consequences. Chronic constipation cannot be cured by this method any more than by the use of cathartics. It is only a mechanical means of emptying the bowels, which is undoubtedly preferable to the re- 
tention of fecal matter ; but at the same time it is not a cure of the morbid condition, and a person who habitually uses the enema becomes as much dependent upon it as is the habitual user of mineral water or pills, upon these agents.

A person who has become dependent upon the enema as a means of moving the bowels in consequence of following the erroneous teachings of Dr. Hall, may find relief by the employment of the graduated enema (24), massage of the bowels (30), and the Swedish movements recommended for constipation (43), together with the adoption of other measures which are elsewhere recommended for the relief of constipation (see page 279).

It may be added that the author's exposure of Dr. Hall's fraudulent claims resulted in destroying his nefarious business, in this country at least, but he succeeded in pocketing a hundred thousand dollars or more before his business was interrupted by the publication of his so-called "secret." 


\section{GRAPHIC METHOD.}

Explanation of the Author's Graphic Mode of Representing the Results of Stomach Work $\mathrm{Ob}=$ tained from the Examination of the Stomach Fluid by the System of Investigation Employed in the Hygienic and Physiological Research Laboratories of the Sanitarium at Battle Creek, Ilich., U. S. A.

In this scheme for the graphic representation of the results of the analysis of stomach fluids (Plate $\mathrm{XI}$ ), the first five columns relate to the work done by the stomach in as many different phases of its digestive and chemical activity. The remaining columns are devoted to the coefficients of digestive work, by reference to which one may see at a glance the relation of the work done, in quantity or quality, to the normal standard. The figures in the several columns represent, at the bottom, the lowest figures, and at the top, the highest figures, which have been observed in the examination of more than five thousand stomach fluids. The figures included in the dark-colored band which passes across the center of the chart, represent the normal limit. The significance of the figures shown in the several columns is as follows :-

The letter $\mathbf{A}^{\prime}$ indicates the total work dene by the stomach in setting free the hydrochloric acid necessary 


\section{GRAPHIC REPRESENTATION}

Of the Results of the Chemical Examination of ISalivary and Gastric Digestion, Based upon the Study of over 5000 Cases in the Physiological Laboratory of the Battic Creek (rich.) Sanitarium.

Case of

AAA ANGEO BT

J. H. KELLOGG, M. D.

\begin{tabular}{|c|c|c|c|c|c|c|c|c|c|c|c|}
\hline \multirow{3}{*}{$A^{\prime}$} & \multirow{3}{*}{$\underset{\text { Free HCl. }}{\mathbf{H}}$} & \multirow{3}{*}{$\begin{array}{c}\mathbf{C} \\
\text { Combined } \\
\text { Chlorine. }\end{array}$} & \multirow{3}{*}{$\begin{array}{c}\text { A } \\
\text { Total } \\
\text { Acility. }\end{array}$} & \multirow{3}{*}{$\underset{\substack{\text { Starch } \\
\text { Digestion. }}}{\mathbf{S}}$} & \multicolumn{7}{|c|}{ COEFFICIENTS OF DIGESTIVE WORK. } \\
\hline & & & & & \multirow{2}{*}{\begin{tabular}{|l} 
Chiorine \\
Liberation. \\
$\mathbf{m}$
\end{tabular}} & \multicolumn{2}{|c|}{ Fermantation. } & \multirow{2}{*}{ 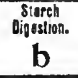 } & \multirow{2}{*}{$\begin{array}{l}\text { Shatiury. } \\
\text { inturity. } \\
\text { C }\end{array}$} & \multirow{2}{*}{$\begin{array}{c}\text { solvian. } \\
y\end{array}$} & \multirow{2}{*}{$\begin{array}{c}\text { Alsoseption. } \\
\mathbf{Z}\end{array}$} \\
\hline & & & & & & a & $\mathbf{x}$ & & & & \\
\hline .430 & .240 & .410 & .480 & 7.00 & 2.00 & 6.00 & 100 & 2.00 & 10.00 & 2.00 & 6.00 \\
\hline .410 & .225 & .390 & .440 & 6.50 & 1.90 & 5.00 & so & 1.00 & 9.00 & 1,90 & 5.00 \\
\hline .390 & .210 & .370 & .410 & 6.25 & 1.80 & 4.50 & 60 & 1.80 & 8.00 & $1 ; 50$ & 4.00 \\
\hline $.37 Q$ & 195 & .350 & .385 & 6.00 & 1.70 & 4.00 & 50 & 1.70 & 7.00 & 1,70 & 8.00 \\
\hline $.350^{\circ}$ & .150 & .330 & .360 & 5.75 & 1.65 & 3.50 & 40 & i. $\oint_{5}$ & 6.00 & $1.65 \%$ & 2.50 \\
\hline $.335^{\circ}$ & .165 & .315 & .340 & 5.50 & 1.80 & 3.00 & 30 & 1.69 & 5.50 & 9.60 & $2.25 \mathrm{~s}$ \\
\hline .320 & 1150 & 300 & 320 & 5.25 & 1,55 & 2.75 & 扬 & 1.50 & 5.00 & 1.550 & $2.00 \mathrm{~g}$ \\
\hline .305. & $: 135$ & , 285 & 305 & 5.00 & $1: 50$ & 2.50 & 30 & $1.50^{\circ}$ & 4.50 & $118^{\circ}$ & 1.750 \\
\hline .290 & .1200. & 8280 & .290 & 4.75 & 40 & 2.25 & $\beta$ & 1.45 & 4.00 & & 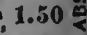 \\
\hline .275 &.$i \not \alpha$ & & 275 & 4.50 & 1.4d & {$[2.00]$} & 6 & 1.40 & & 1.40 & $1.40=$ \\
\hline .260 & . 100 & .240 & $.260^{\circ}$ & 4.25 & C.35! & 1.75 & 4 & 4.35 & Broo & 1.35 & 4.35 \\
\hline .245 & .090 & .225 & .245 & 4.00 & 1.30 & $|1.50|$ & (2) & $1+30$ & 850 & 1.30 & $1.30 \%$ \\
\hline .230 & .080 & .210 & .930 & 3.75 & :1.25 & 1.40 & 60 & 1.25 & /2.po & 1.25 & I,95 \\
\hline .220 & .070 & .200 & .220 & 3.50 & $: 1.20$ & 1.30 & 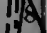 & 1.20 & 1. 5 & 1.20 & 120 \\
\hline .210 & .060 & .190 & .210 & 3.25 & 1.15 & 1.20 & 16 & 1.15 & 1.010: & 1.15 & 1.15 \\
\hline .200 & .050 & .180 & .200 & 8.00 & 1.10 & 17.10 & 4 & 1.10 & $1.2 \%$ & 1.10 & 1.10 \\
\hline . 195 & .044 & .0174 & .195 & 2.75 & 1.05 & 1.051 & 2 & $10 *$ & 1,18 & 1.05 & 105 \\
\hline .1010 & Iass & V.Jug & .190 & 20001 & 1.09 & $\angle 00$ & (10) & 1.00 & 1.00 & 1.00 & 1.20 \\
\hline .155 & .031 & 161 & .185 & 9.65 & .95 & $\sqrt{2}$ & : & .95 & .901 & -45 & .35 \\
\hline .180 & .025 & .155 & .180 & 200 & .90 & $\vdots .9$ & & .90 &.$\$ 0$ & $.8 p$ & .90 \\
\hline 170 & .024 & .145 & .170 & $7 ! 891$ & .85 & 1.9 & & .85 & :70 & .83 & .85 \\
\hline .160 & .023 & .135 & .160 & 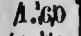 & .80 & $: 00$ & & .80 & $\therefore 60$ & 80 & .80 \\
\hline . 150 & .022 & .125 & . 150 & $11 . \% 0$ & 1.75 & 47 & $4 \mathrm{E}$ & $: 75$ & (50. & .75 宸 & .75 \\
\hline .140 & .021 & .115 & .140 & 1.20 & 1.70 & 17 & & : & .40 & $.70 \mathrm{~g}$ & ${ }^{70} \dot{z}$ \\
\hline .130 & .020 & .105 & $.130 /$ & 1.00 & $i^{65}$ & & 4 & $.4 x^{\circ}$ & .30 & .65 品 & $.65 \%$ \\
\hline 120 & sors & [. & 8129 & .90 & - $0^{\prime}$ & & $d=$ & .60 & .25 & $.60 \frac{\bar{z}}{n}$ & $.60 \%$ \\
\hline .510 & .0113 & 1085 & .140 & .80 & .36 & & 3. & .55 & .20 & $.55 \frac{\partial}{0}$ & 55 \\
\hline . 100 & .014 & 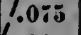 & 100 & .70 & .50 & & 1. & .50 & .15 & !50 & 150 ? \\
\hline .085 & .012 & .065 & .085 & .60 & .45 & & 3ी है। & .45 & .12 & 450 & .453 \\
\hline .070 & $<010$ & .05\% & .070 & .50 & .40 & .4 & (읭 & .40 & .10 & 40 焉 & $.40 \mathrm{~s}$ \\
\hline .055 & .0os & .045 & .055 & .40 & .35 & .3 & & .35 & .08 & $.15 \mathrm{~g}$ & 83 \\
\hline .040 & .036 & .035 & .040 & .30 & .30 & & & .30 & .06 & .505 & .30 \\
\hline .025 & .004 & .025 & .025 & $1.20^{\prime}$ & .20 & .2 & & .20 & .04 & $.20^{\circ}$ & .20 \\
\hline .010 & 002 & 010 & .010 & .10 & 10 & .1 & & 10 & .02 & .10 & .10 \\
\hline .000 & & & .000 & .00 & .00 & .0 & & .00 & .00 & .00 & .00 \\
\hline & & & & & $\mathbf{m}$ & $\mathbf{a}$ & & b & c & $\mathbf{y}$ & $\mathbf{z}$ \\
\hline
\end{tabular}

PIATE NI. 


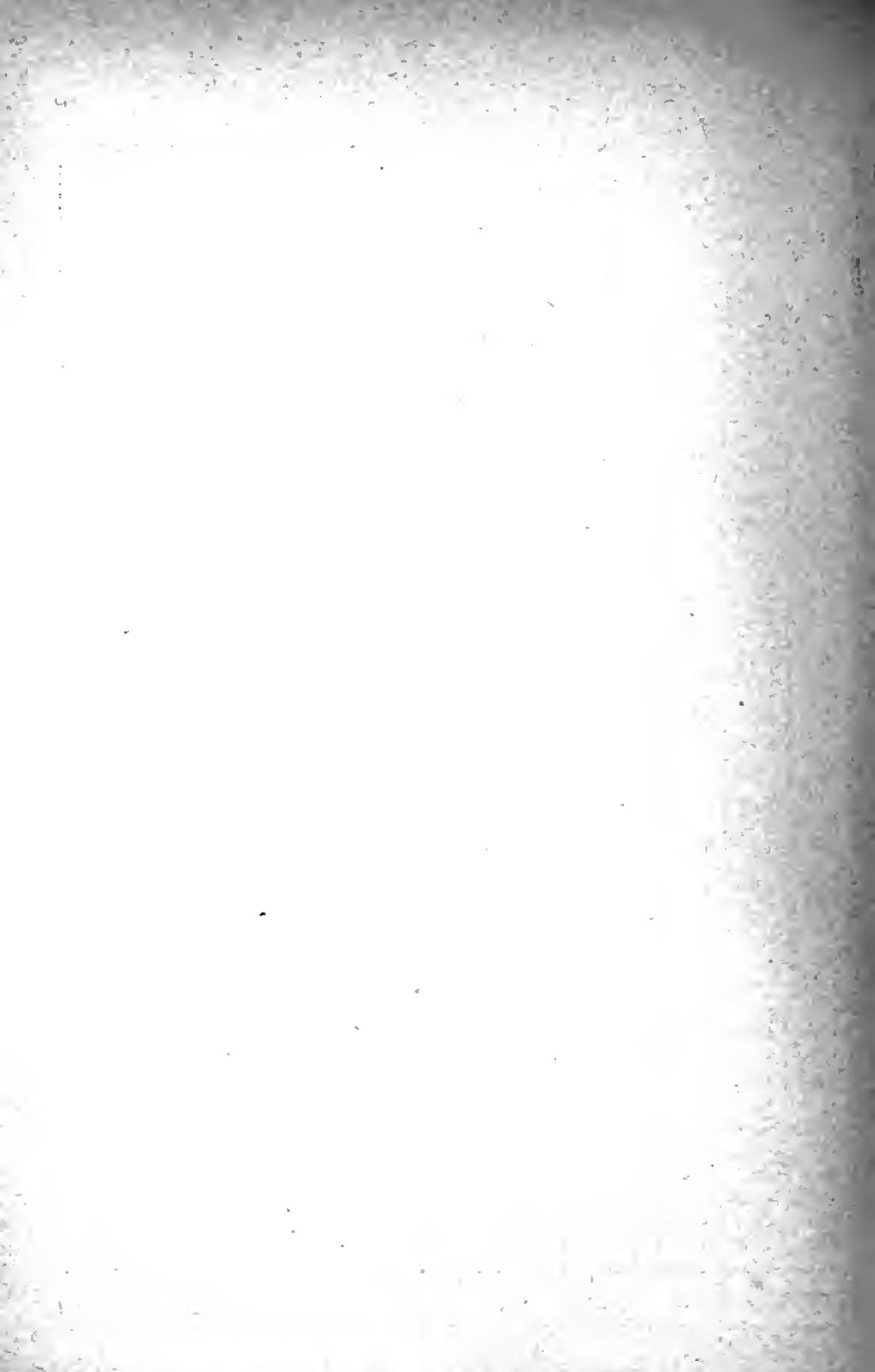

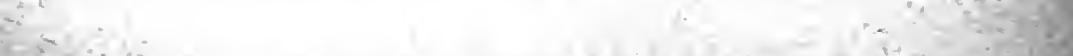

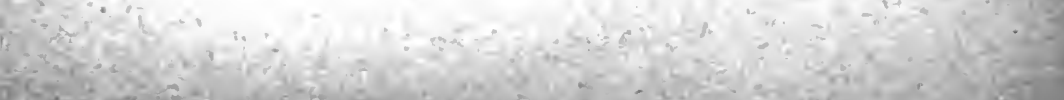

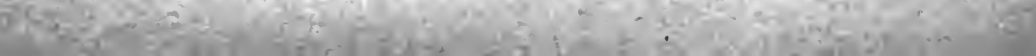


for digestion. If the figures found by analysis fall below the normal limits, that is, below .180, the case is one of hypopepsia; in other words, the stomach does less than the normal amount of work. If the zero point is reached, the case is one of apepsia, and the stomach is doing no digestive work whatever. If the figures fall above the normal limit, that is, above .200 , the case is one of hyperpepsia ; in other words, the stomach does more than the normal amount of work.

In the treatment of apepsia and hypopepsia, all proper means are used to increase the activity of the gastric glands, thereby supplying the deficiency of hydrochloric acid. The principal means to be adopted are, heat over the stomach, massage, electricity, the moist abdominal bandage, exercise after eating, a hot and cold douche over the stomach.

In cases of hyperpepsia, or excessive formation of hydrochloric acid, the aim of the treatment administered is to lessen the glandular activity of the stomach. It should consist of hot sponging of the spine; an ice bag over the stomach in extreme cases; galvanism to the spine, the stomach, and the pneumogastric nerve; and the avoidance of all stimulating foods and condiments.

The figures of the second column, $\mathbf{H}$, relate to the amount of free hydrochloric acid present in the stomach fluid. When it is remembered that free $\mathrm{HCl}$ is necessary, not only as an aid to digestion, but as a means of preventing the growth of germs and the production of poisonous substances through the decomposition of the food, the importance of this determination will be ap- 
preciated. Frec hydrochloric acid may be absent, deficient, or present in either normal quantity or in excess, in both hypopepsia and hyperpepsia, as well as in cases in which the total amount of work done by the stomach is normal, the chief departure from the normal standard being some form of acid fermentation.

Column A, which has relation to the total acidity of the stomach contents, has the same normal limit as $\mathbf{A}^{\prime}$. Outside of the normal limits, however, there is often a wide contrast between the figures shown in these two columns. The total acidity may be either greater or less than the sum of the free hydrochloric acid $(\mathrm{HCl})$ and the combined chlorin (C). When greater, the indication is that acids are present in the stomach which have been formed by fermentation. When less than the normal amount, the indication is that the products of digestion are of an inferior quality.

A deficiency of free $\mathrm{HCl}$ requires stimulation of the gastric glands, and in some extreme cases benefit is derived from the administration of small quantities of $\mathrm{HCl}$ with a little diluted water. When the $\mathrm{HCl}$ is in excess, patients often suffer pain or sympathetic nervous disturbances. The total acidity, $\mathbf{A}$, compared with $\mathbf{A}^{\prime}$, is a good means of determining the quality of the digestive work done.

Column $\mathbf{S}$ represents the quality of maltose, or digested sugar, found in the stomach for each 100 c.c., or three and one half ounces, of stomach fluid. A deficiency indicates either an excessively acid gastric juice or insufficient mastication of the food. When the figures found are above normal, the indication is that the 
activity of salivary digestion has been unusually great. This is a condition from which no harm can arise.

The facts which the author's researches have elicited respecting starch digestion are, for the most part, new. They are, nevertheless, important, and have excited considerable interest.

\section{The Coefficients of Digestive Work. - Of} the several coefficients of digestive work, coefficient a is one of the most valuable and important. When below 1.00 , this coefficient indicates an inferior quality in the digestive product. When above 1.00, the indication is that there are present other acids than $\mathrm{HCl}$ and combined chlorin.

Coefficient $\mathbf{b}$ relates to starch digestion. If the figures found by analysis fall below the normal limit, 1.00 , the indication is that a smaller amount of sugar is formed by the action of saliva upon the starch than in the normal state. This difficulty may commonly be remedied by the use of dry food, which naturally secures thorough and prolonged mastication.

The figures falling below 1.00 in column $\mathrm{e}$ indicate a deficient secretion or a deficient quality of saliva. Those found above the normal line indicate an unusual activity or abundance of salivary secretion.

Coefficient $\mathbf{y}$ has relation to the disintegration or division of food into fine particles. When disintegration is deficient, as shown by figures below 1.00 , the indication is for the use of dry food and thorough mastication. Granose is admirably adapted to these cases.

Low figures under coefficient $\mathbf{z}$ represent very slow absorption. Figures which fall above 1.00 indicate an unusually rapid absorption. 
The two last-mentioned coefficients, - those relating to disintegration and absorption,- especially the lastnamed, or coefficient $\mathbf{z}$, are less exact than are the other coefficients, yet are of considerable value, if pains is taken to obtain the whole quantity of fluid contained in the stomach at the end of an hour after the test meal is taken.

Coefficient $\mathbf{m}$ has relation to the work of the stomach in producing free hydrochloric acid to take part with pepsin in the digestion of food. In hypopepsia, too little chlorin is set free ; in hyperpepsia, too much ; in apepsia, none at all. Improvement in hyperpepsia will be indicated by a lowering of the figures representing this coefficient; in hypopepsia and apepsia, the reverse.

The indications derived from these coefficients are as follows :-

Coefficient a indicates the necessity for improve. ment in the quality of digestive work done. Electricity, massage, manual and mechanical Swedish movements, and a suitable dietary, especially foods containing lactic acid, as kumyss or kumyzoon, buttermilk, etc., are necessary. Malted gluten and granose have also proved very serviceable in improving the quality of the digested products.

A high coefficient $\mathbf{x}$, or $\mathbf{a}$ and $\mathbf{x}$, indicates fermentation. A dry diet, consisting chiefly of granose and fruits, with the use of antiseptic tablets, is generally required in these cases. Stomach washing by means of lavage is also required.

A low coefficient $\mathbf{b}$ indicates the necessity of more thorough mastication or the withdrawal of starchy food 
from the dietary. In these cases a fruit-and-nut diet or the use of malted gluten - is found to be of very great service. In some cases a diet consisting chiefly of kumyzoon, kumyss, buttermilk, eggs, and similar foods must be resorted to for a time. A high coefficient $\mathbf{b}$ requires no attention.

A low eoefficient c may result from gum-ehewing or tobaeco-using, the eonstant spitting having the result of weakening the salivary glands. The application of eleetricity to the back of the neck and at the pit of the stomach is often useful in these cases.

A low eoefficient $\mathbf{y}$ indicates a demand for more thorongh mastieation, and perhaps the use of some thoroughly disintegrated food, as granose, for a short time.

Coefficient $\mathbf{z}$, if low, indieates the necessity of abstaining from the eating of liquid foods and drinking at meals. Special massage or manual Swedish movements must be adopted as a means of increasing the motor activity of the stomach. Electricity is often useful in this elass of eases. 



\section{INDEX.}

Abdomen, galvanization of, 328 .

Abdominal bandage, the dry, 3:0.

Abdominal contents, inspiratory lifting of, 307 .

Abdominal massage, 304 .

Abdominal muscles, exercises to develop, 311.

Abdominai supporter, 329 .

Absinthe, French, 347 .

A bsorbents, 28.

Acid, hydrochioric, $\mathbf{2 6}, \mathbf{1 6 3}, 323$.

Acid, lactic, 26, 323 .

Acidity, 147, 16:.

Acidity, calculated, 145.

Acidity from hy perpepsia, 163.

Acidity, total, 145.

Acne, 190.

Albumen, 33.

Aicohol, 114.

Alimentary canal, 21.

Alkalies, 119.

Almond, sweet, nutritive value of, 42.

Alum, 120.

Ammonia, 120.

Amylopsin, 30.

Anai itching, 153.

Anemia, Swedish movements for, 315.

Animal foods, 31, 35 .

Anti-fat dietary, 232 .

Antifebrin, 197.

Antipyrin, 197.

Apepsia, 142, 260 .

Aphthæ, 181.

Apoplexy, nervous, 200.

A ppetite, excessive, 152 .

A ppetites, perverted, 120.

Apple, dried, nutritive value of, 41 .

Apple, nutritive value of, 41.

Apples, time of digestion of, 40 .

Apricot, nutritive value of, 41 .
Aqua salina, 324 .

Aseptic dietary, 227.

Asparagus, nutritive value of, 4 . Assyrians, diet of, 39 .

Asthma, nervous, 154.

Asthma of indigestion, nocturnal, 203.

Backache, 20, 186.

Baker's bread, 9:.

Baking-powders, 119.

Banana, nutritive value of, 41 .

Barley meal, 93.

Barley, nutritive value of, 41 .

Bariey, South Russian, nutritive value of, 41.

Barley, time of digestion of, 40.

Bathing after mea is, 78 .

Beans, field, nutritive value of, 42 .

Beans, French or kidney, nutritive value of, 42 .

Beans, Lima, nutritive val ne of, 42.

Beans, pod, boiled, time of digestion of, 40 . .

Beans, string, nutritive value of, 42 . Beans, white, nutritive value of, 42 . Beating, 15:, 308.

Beaumont, Dr., 109.

Beef, fat, 93.

Beef, lean, 93.

Beef, lean, fried, time of digestion of, 40 .

Beef, lean, nutritive value of, 42 .

Beef, lean, rale roasted, time of digestion of, 40.

Beef, salted, boiled, time of digestion of, 40.

Beefsteak, broiled, time of digestion of, 40.

Beef tea, 99.

Beer, 92.

Beet, nutritive value of, 42. 
Beet, sugar, nutritive value of, 42 . Belching, $15 \%$.

Benzoin solution, 325.

Bernard, 102.

Bicycle riding, 124, 199.

Bile, 29.

Bile, diminished quantity of, 10 2.

Biliousness, 81, $17 \%$.

Biscuit, 161.

Bismuth, subcarbonate of, 325.

Bismuth, subgaliate of, 326 .

Blackberry, nutritive value of, 41 .

Bouchard, 19, $\tilde{70}, 99$.

Bowels, massage of. 30 .

Bowels, prolapsed, Swedist movements for, 317 .

Brain work, 124.

Bread, barley, nutritive value of, 41.

Bread, corn, time of digestion of, 40 .

Hread, fine-flour, 91.

Bread, raised, 161.

Bread, rye, nutritive value of, 41 .

Bread, swedish speise brod, nutritive value of, 41.

Bread, wheateu, time of digestion of, 40.

Bread, white, nutritive value of, 41 .

Bread, whole-wheat, nutritive value of, 41.

Breads, unfermented, 235 .

Breath, shortness of. 154 .

Bright's diseise, 18, 108, 190.

Brinton, Jr., 195.

Bromose, 241.

Brown's Iron Bitters, 346.

Buckwhent, nutritive value of, $: 1$.

Burkart, 194.

Burning, 152, 153, 154.

Butter, 35, 101, 103, 105.

Butter, French, nutritive value of, 42 .

Butter, melted, time of digestion of, 40 .

Buttermilk, nutrltlve valne of, 42 .

Butter, Swedish, nutritive value of, 42.

Butyric acid, 164.

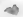

Cabbage, 92.

Cabbage, boiled, time of digestion of, 40.
Cabbage, raw, time of digestion of, 40.

Cabbage, red, nutritive value of, 42.

Cabbage, white, nutritive value of, 42.

Cabbage, winter, nutritive value of, 42 .

Caffein, 114.

Cancer of liver, 184.

Cancer of stomach, 277.

Cane-sugar, 33.

Caramei-cereal, 241.

Cardamoms, 109.

Carrot, nutritlve value of, 42 .

Carrots, 92, 93.

Carter's Little Liver Pills, 348.

Cascara sagrada, 323 .

Casein, 33.

Catarrh, gastric, 184.

Catarrh, gastric, diet for, 238 .

Catarrh, intestinal, 184.

Catarrh of stomach, 26.5.

Catarrh of stomach. ehronic, $2 \%$.

Cauliflower, nutritive value of, 42.

Celery, nutritlve value of, 4 .

Cellulose, 34 .

Chair, vibrating, 335.

Chalk, 121.

Charcoal, 322.

Charcoal tablets, 322 .

Charcoal tablets, antiseptic, 323 .

Cheese, 35, 100, 161.

Cheese, Stilton, nutritive value of, 42.

Cheese, time of digestion of, 40 .

Cherry, dried, nutrltive value of, 41.

Cherry, nutritive value of, 41 .

Chestnut, nutritive value of, 42 .

Chlcken soup, boiled, time of dlgestion of, 40.

Child, food for a, 236.

Chinese, diet of, 39.

chlorids, fixed, 145 .

Chiorin, combined, 145.

Chlorin, total, 145.

Cinnamon, 109.

Cinnamou solution, 324.

Circulation, disturbance of, 202.

Cirrhosis of liver, 184 . 
Clapotement, 131.

Classification of digestions, 142.

Clay-eaters, 120.

Cloves, 109.

Cocoanut, nutrltive value of, 42 .

Coeffieients, 139, 355 .

Coldness, 152.

Colic, 171.

Coloelyster, 30 .

Colon, the, 28.

Colon, the, a reservoir, 73 .

Colors, unnatural, 154.

Compression, inspiratory, 306.

Condiments, 34, 109.

Confectionery, 67.

Cookery, bad, 94 .

Cooking, 98.

Constipation, 69, 153, 184, 279 .

Constlpation, diet for, 238 .

Constipation, Swedish movements for, 319.

Constriction, 152, 153, 172.

Consumption, 18.

Consumption, how eaused, 19.

Corn and beans, green, time of digestion of, 40.

Cornaro, 88.

Corn, dent, nutritive value of, 41 .

Corn, flint, nutritive value of, 41 .

Corn-starch or arrow-root gruel, 91.

Corn, sweet, nutritive value of, 41 .

Corsets, 123.

Cow-tree, milk of, nutritive value of, 42.

Cranberry, nutritive value of, 41 .

. Cream, 104.

Oream, nutritive value of, 42.

Cream tartar, 119.

Cueumber, nutritive value of, 42.

Cucumbers, 111.

Currant, nutritive value of, 41 .

Date, dried, nutritive value of, $\mathbf{t 1}$.

David, 19.

Debove, 261.

Dentifrice, antiseptic, 324.

Depression, 20, 153.

Desserts, 87.

Dextrin, 97.

Dlabetes, 107.

Diabetes, diet for, $\mathbf{2 3 7}$.
Diarrhea, 153, 185.

Dickens, Charles, 85.

Diet and regimen, 224.

Dietetic rules, 224 .

Diet, natural, 39.

Diet tables, 206.

Diet, unseasonable, 122.

Digestion, 21 .

Digestion, allowing time for, 74 .

Digestion during sleep, 70,72 .

Digestibility of various foods, table of, 40.

Dilatation of stomach, 19,81 .

Dinners, six o'clock, 71 .

Discharges, the, 153.

Discontent, 124.

Distention, 15:.

Douche, liver, 300 .

Douche, pail, 300.

Dress, healthful, 341 .

Drowsiness, $87,15 \% 3$.

Drowsiness, unusual, 211.

Drugs, 125.

Dry dietary, 228.

Duck, roasted, time of digestion of, 40.

Dujardin-Beaumetz, 70 .

Dyspepsia, acid, 249 .

Oyspepsia, bilious or foul, :251.

Dyspepsia, home treatment of, 287 .

Dyspepsia in America, 17.

Dyspepsia, infantile. $2 \%$ :

Dyspepsia, inherited, $12 \%$.

Dyspepsia, nervous, 257 .

Dyspepsia, paiuful, 223.

Dy spepsia, simple, :24.

Dy spepsia, symptoms of, 149

Dyspepsia, Swedish movements for, 318 .

Dyspepsia, treatment of, 221 .

Ears, ringing in, 154.

Eating between meals, 67 .

Eating late at night, 71 .

Eating too frequently, 66.

Eating too little, 82.

Eating when exhausted, 73.

Economy of vegetable food, 89.

Eczema, 190.

Eels, vinegar, 113.

Egg, entire, nutritive value of, 42.

Eggs, 36, 9:2, 93, 23:2. 
Eggs, frled, tIme of digestion of, 40 . Eggs, hard boiled, time of digestlon of, 40.

Eggs, raw, time of digestion of, 4 .

Eggs, soft bolled, time of digestion of, 40.

Eggs, whipped, tlme of digestion of, 40.

Egg, white of, nutritive value of, 42 .

Egg, yolk of, nutritlve value of, $4:$.

Egyptians, diet of, 39.

Enema, 301 .

Enema, cold, 302.

Enema, graduated, 302 .

Enema, laxative, 303.

Enema, oil, 304.

Enemata, nutritive, 240.

Eno's Fruit Salt, 348.

Epilepsy, 208.

Eructations, 153.

Esophagus. 25.

Esophagus, symptoms pertaining to, 152 .

Exercise, :309.

Exercise, lack of, 1:2.

Exercises to develop abdominal nuscles, 218.

Extracts, fia voring. 1:2

Extremities, cold, 20 .

Falntness, 66, 153 .

Faradic electricity, general applications of, 3\%\%.

Fasting, 339 .

Fat, 40.

Fat and blood dletary, 233 .

Fats, 33.

Fats, abundant use of, 101.

Feet, cold, 191.

Fermentation, 148, 159.

Fever diet, 234 .

Fidgets, 153.

Fig, dried, nutritlve value of, 41 .

Fish, 36.

Fish, white, nutritive value of, 42.

Flatulence, 156, 163.

Flatulence, intestInal, 157.

Flesh, 36.

Flesh food, 242.

Flesh food, excessive use of, $10 \pi$.

Flour, arrow-root, nutritive value Qt, 41 ,
Flour, banana, nutritive value of, 41.

Flour, barley, nutritive value of, 41.

Flour, bean, nutritive value of, 41 . Flour, buek wheat, nutritlve value of, 41.

Flour, corn, nutrltive value of, 41 . Flour, famlly, 91.

Flour, graham, nutritive value of, 41.

Flour, oat, nutritive value of, 41 .

Flour, patent, 91.

Flour, pea, nutritive value of, 41.

Flour, rye, nutritive value of, 41 .

Flours, gluten, 92.

Flour, wheat, nutritive value of, 41.

Flour, whole-wheat, 91.

Fomentation, 295.

Fomentation, mustard or turpentine, 296.

Food, adulterations of, $1:$.

Food combinations, 77 .

Food, decayed, 98.

Food elements, 32.

lood elements, classification of, 32 .

Food elements, deficiency In, 89.

Food, fermentation of, 19.

Food for brain laborer, 84.

Food for muscle laborer, 84 .

lood, fried, 95.

Food, quantity of, 79 .

Food producers, 31.

Food, proper quantity of, 85 .

Food, quallty of, 94.

Food, salted. 111.

Food, soft, 100.

Food, too many varletles of, 76 .

Food, uncooked, 96

Foods, 31, 34 .

Foods, fermenting or decomposing, 239.

Foods, raw, 96.

Foods, sterllized, 227 .

Foods, vegetable, 31, 37 .

Foot bath, 297.

Fowl, 36.

Fowl, bolled, time of digestlon of, 40.

Frogs, 37.

Fruit, green, 97 , 
Fruits, 37, 233.

Full bath, 290.

Full bath, hot and cold, 301 .

Gall-bladder, the, 29.

Gall-stones, 184.

Galvanization of spine and abdomen, 328.

Galvanization of sympathetic nerve, 329 .

Game, 36.

Garfield Tea, 349 .

Gastric juice, 26.

Gastric juice, excess of, 163.

Gastritis, diet for, 238 .

Georges, 261.

German Bitters, 347 .

Germs in water, 129.

Ginger, 34.

Girdle, wet, 294.

Glands, peptic, 25.

Glenard, Dr., 216.

Glucose, 33.

Gluten, 35.

Gluten biscuit, 241 .

Gluttouy, 80.

Gofio, 241.

Gooseberry, nutritive value of, 41.

Goose, roasted, time of digestion of, 40.

Gormandizing, 7 .

Gout, 108.

Graham flour, 9:.

Granola, 240.

Granose, 240.

Grape, nutrltive value of, 41 .

Grape-sugar, 33.

Graphic method, 352.

Gravies, 103.

Greeks, dlet of, 39.

Griddle-cakes, 104.

Gripes, 171.

Guaiac solution, 325.

Hacking, 308.

Hall's so-called “Secret," 349.

Hamburg Tea, 348.

Hamburg Drops, 349.

Hand bath, 289.

Hazelnut, nutritive value of, 42 .

$\mathrm{HCl}$, free, 145.

Heađache, 20, 153, 192.
Headache, nervous, 193.

Health foods, 240.

Hearing, disturbances of, 209.

Heartburn, 164.

Heat, dry, 296.

Heaviness, 152, 153.

Hiccough, 154.

Hip bath, 297.

Holloway's Pills, 349.

Home treatment of dyspepsia, 257.

Honey, nutritive value of, 42 .

1 Iop Bitters, 346.

Horseback riding, 124.

Hostetter's Bitters, 346 .

Hot-air bath, 299.

Hunger, 152.

Hunger-cure, 339.

Hydrochloric acid, $26,163,3: 3$.

IIyperpepsia, 142, 264 .

Hyperpepsia, acidity from, 163, 323.

Iypochondria, 173 .

Hypopepsia, 142, 260 .

Hysteria, 208.

Iceland moss, nutritive value of, 41 .

Indian meal, 9?, 93.

Indigestible elements, 34 .

Indigestion, intestina $1,268$.

Indigestion, septic, 251 .

Indigestions, classification of, 1.00.

Indigestions, symptoms of, 151.

Infant, food for a bottle-fed, 23\%.

Infant, food for a teething, 236 .

Infantlle dyspepsia, 272.

Insanity, 18.

Insomnia, 153.

Intestinal indigestion, 268.

Intestinal juice, 28.

Intestine, the large, 28.

Irritability, 153.

Japanese, diet of, 39 .

Jaundice, 18, 184.

Jaundice, catarrhal, $10 \%$.

Jolnt movements, 308.

Kidneys, prolapsed, Swedish movements for, 317.

Kneading, 304.

Kneading, mechanical, 335,

Kumyzoon, 241, 
Lactle acid, 26.

Lamb, broiled, time of digestion of, 40.

Lapps, 16.5.

Lard, 101.

Lavage, 320 .

Lavage, hot and cold, $3 \mathrm{x} 2$.

Laxatives, 125, 323.

Lean meat, 92 .

Lee's Anti-Billous Pills, 348.

Lentils, German, nutritive value of, 4 .

Lentils, nutritive value of, 4 .

Letheby, 88 .

Lettuce, nutritive value of, 42 .

Liquid dietary, $: 34$.

Liver, :99.

Liver, cancer of, 184.

Liver, eir rhosis of, 184.

Liver pills, 12\%.

Liver, torpid, $\mathbf{2 6 9}$.

Lobsters, 37 .

Macaroni, nutritive value of, 41 .

Malarial disease, 18.

Malt sugar, or maltose, 97 .

Man a frugivorous animal, 96.

Manna, uutritive value of, 41 .

Mlassage, 304 .

Massage, abdominal, 304 .

Massage, cannon-ball, 337.

Massage, general, 308.

Massage of bowels, 305 .

Massage of stomach, 306.

Meals, bathing after, 78 .

Meals, eating between, $6 \pi$.

Meals, irregularity of, 67 .

Meals, proper number of, 69.

Meals, sleeping after, $\%$.

Meals, too Prequent, 66.

Meat, "high," 161.

Meat, raw, 108.

Meats, canned, 98.

Mechanical kneading, 335.

Melon, nutritive value of, 42.

Memory, loss of, 153.

Mental impressions, 124.

Mental confusion, $15 \tilde{}$.

Mental disorders, 214.

Mental Iabor, 124.

Mental treatment, 344.

Mexico, 110.
Nicrobes of the mouth, 19.

Migraine, 177, 193.

Mlgranin, 197.

Nilk, 35, 92, 93, 231.

Milk and meat, 78 .

Milk and vegetables, 78 .

Milk, boiled, time of digestion of, 40.

Milk, cow's, nutritive value of, 42 .

Milk-curdllng ferment, $\mathbf{3 0}$.

Milk, mother's, nutritive value of, 42.

Miik of cow-tree, nutritive value of, 42.

Milk, raw, time of digestion of, 40 . Nilk, skimmed, nutritive value of. 42.

Milk-sugar, 33.

Millet, nutritive value of, 41 .

Mineral waters, 120.

Moral treatment, 34 .

Mosso, Dr., 261.

Motor functions, disturbances of, 153.

Moutb, 22.

Mouth, antisepsis of, 242.

Mouth, sour taste in, 163.

Nouth, symptoms pertainiug to, 152.

Ilustard, 34, 109.

Mutton, broiled, time of digestion of, 40.

Mutton, lean, nutritive value of, 4.2.

Iutton, roasted, time of digestion. of, 40.

Natural Abdominal Supporter, 320.

Nausea, 153, 166.

Nervous diseases, 210.

Nervousness, $20,153,208$.

Nervous symptoms, 207.

Neurasthenia, 108.

Neurasthenia, gastric, 257.

Newton, 85.

New Zealanders, 165.

Niemyer, Dr., 195.

Nitrogenous dietary, 229.

Nostrums, 345.

Numbness, 154.

Nutritive treatment, general, 331. 
Nut butter, 241.

Nut cream, 105.

Nut meal. 241.

Nuts, 104, 233, 234.

Oatmeal, 92, 93 .

Oats, nutritive value of, 41 .

Obesity, $10 \%$.

Oll rubbing, 304.

Olis, vegetable, 33.

Onion, nutritive value of, $4 \mathrm{~s}$.

Overeatlng, 80.

Oysters, 37, 161.

Oysters, raw, time of digestion of, 40.

Oysters, stewed, time of digestion of, 40.

Pain, 153, 173.

Palpitation of the heart, 154.

Parkes, 88.

Parsnip, nutritive value of, 42.

Parsnips, 92.

Parsnips, boiled, time of digestion of, 40.

Pasteur, 19.

Pastry, 90.

Peach, nutritlve value of, 41 .

Peach pickles, 111.

Peanut, nutritive value of, 42.

Pear, dried, nutritlve value of, 41.

Pear, nutritive value of, $\mathbf{4 1}$.

Peas, 82, 93.

Peas, African, nutritive value of, 42.

Peasants, diet of, 39.

Peas, green, garden, nutritive value of, 42 .

Peas, green, shelled, nutritive value of, 42 .

Peas, small, nutritive value of, 4 .

Peivis tilting, 336.

Pepper, 34, 109.

Pepper, Dr., 194.

Pepper-sauce, 34, 109.

Pepsin, 19.

Pepsin, what about? 261.

Peptic glands, 25 .

Peptogens, 38.

Peptones, 19.

Perfection Vaporizer, 325.
Pickles, 111.

Pie-crust, 103.

Plants, 31.

Plants, pod-bearing, 38.

Pleasant Purgative Pellets, 349.

Plum, nutritive value of, 41 .

Pork, 36.

Pork, nutritlve value of, 42 .

Pork, roasted, time of digestion of, 40.

Pork, saited, fried, time of digestion of, 40.

Portal circulation, 51.

Positions, bad, 123.

Potatoes, 92, 93.

Potatoes, Irisb, baked, time of digestion of, 40.

Potatoes, Irish, boiled, time of digestion of, 40.

Potato, nutritive value of, 42 .

Potato, sweet, nutritive value of, 42.

Poultry, nutritive vaiue of, 42 .

Pour, hot and cold, 300 .

Prolapsed colon, Swedish movements for, 317 .

Prolapsed stomach, Swedish movements for, 317 .

Prune, dried, nutritive value of, 41.

Pruue, nutritive value of, 41.

Ptomains, 19.

Ptyalin, 22.

Pugilists, 89.

Pumpkin, nutritive value of, 42.

Pylorus, 26.

Pyrosis, 165.

Quaicks, 345.

Quantity of food, 79.

Radway's Regulatlng PIlls, 348.

Raisin, nutritive value of, 41.

Raspberry, nutritive value of, 41.

Rectum, symptoms pertaining to, 153.

Regurgitation, 153, 170.

Rémond, 261.

Respiration, mechanical, 337.

Rest-cure, 329.

Rheumatism, 108. 
Rheumatism and gout, dict for 238.

Rheumatism, chronic, 19.

Rice, $92,93$.

Rice, nutritive value of, 41 .

Rice, time of digestion of, 40.

Roberts, Dr. William, 112.

Romans, diet of, 39.

Rowing, 124.

Rubbing wet-sheet, 292 .

Rumination, or merycism, 153, 170.

Rye meal, 92, 93.

Rye, German, nutritive value of, 41.

Rye, winter, nutritive value of, 41.

Sago, time of digestion of, 40.

Saleratus, 119.

Saliva, 22.

Salivary digestion, 96.

Sallvary glands, 济.

Salmon, nutritive value of, 4.

Salmon, salted, boiled, time of digestion of, 40.

Salt, 109, 110.

Salt glow, 291 .

Salts, 32, 33.

Salted meats, 37 .

Saratoga chips, 104.

Sauerkraut, 161.

Scurvy, 90.

Sée, N. Germaine, 93.

Seltzer, 324.

Sensation of fulness, 152.

Sexual abuses, 126 .

Shell-fish, 37, 99.

Sighing, 154.

Shower bath, 291.

Sight, disturbances of, 209.

Simon's Liver Reguiator, 348 .

Simple dyspepsia, 142 .

Sinking sensation, 1 r3.

Sinusoidal current, high tension, $32 \%$.

Sinusoidal current, low tension, 328.

Sirup, nutritive value of, 42.

Sitz bath, 297.

Skin, dryness of, 190.

Skin eruptions, 190.

Sieeping. 343.
Sleeping after meals. 75.

Sleeplessness, 212.

Small iutestine, 26.

Smoked meats, 37 .

Soda, bicarbonate of, 328 .

Soothing Syrup Mrs. Winslow's, 127.

Soreness, 175.

Soup, bean, time of digestion of, 40.

Soup, marrow-bone, time of digestion of, 40.

Soup, mutton, time of digestion of, 40.

Spinach, nutritive value of, 42.

Spine, galvanization of, 328 .

Spine, heat and cold to, 299.

sponge bath, 289.

Squash, nutritive value of, 42.

Stammering, 153.

Starch, 32, 40.

Starch digestion, 96.

Starch granules, 97.

Starch indigestion, 266.

Starch, raw, 9i.

Steapsin, 30.

St. Martín, 109.

Stomach, 2i, 25.

Stomach Bitters, 347.

Stomach, cancer of, 277 .

Stomach, catarrh of, 265.

Stomach, chronic catarrb of, 275.

Stomach contents, examination of, 134.

Stomach cough, 15t, 205.

Stomach, dilatation of, 19, 81, 215.

Stomach massage, 306.

Stomach, pressure of, 123.

Stomacb, prolapse of, 216 .

Stomach, prolapse. of, Swedish movements for, 317 .

Stomach, symptoms pertaining to, $15 \%$.

Stomach-tube, 130, 321.

Stomach, ulceration of, diet for, 238.

Stomach, ulcer of, 276.

Stomach, uneusiness at, $15 \%$.

Stools, 187.

Stools, painful, 153.

stoughton Bitters, 346.

Strawberry, nutritive value of, 41. 
Strength, eating for, 237.

Suet, 101.

Sugar, $32,40$.

Sugar, use of in excess, 105.

Sulphur, 324.

Sun bath, 298.

Suppers, late, 71 .

Swedish movements, manual, 314.

Swedish movements, mechanical, 334.

Sweetmeats, 67.

Sympathetic nerve, galvanization of, 329.

Symptoms, general nervous, 153 .

Tapeworm, 18.

Tapioca, time of digestion of, 40 . -

Tea drunkenness, 90.

Teeth. 22.

Teeth, permanent, 24.

Teeth, structure of, 100.

Teeth, temporary, 23.

Test meal, 134 .

Thein, 114.

Throat-all, 182.

Throat, symptoms pertaining to, 152.

Tight lacing, 123 .

Tightness, 172.

Tingling, 154.

Tobacco, 117.

Tomato, nutritive value of, 42.

Tomatoes, 111.

Tonic treatment, general, 333.

Tongue, appearance of, 179.

Tongue, coated, 20.

Tonslls, 22 .

Toxins, 19.

Traveling, 343.

Tropic Fruit Laxative, 348.

Trout, time of digestion of, 40 .

Trunk pack, 294.

Trunk pack, hot and cold, 294.

Trunk rolling, 336 .

Trý psin, 30 .

Turkey, domestic, boiled, time of digestion of, 40 .

Turkey, roasted, time of digestion of, 40.

Turnip, white, nutritive value of, 42.

Turnips. 92.
Turnips, flat, boiled, time of digestion of, 40.

Typhold fever, 18.

Tyrotoxicon, 35.

Uicer of stomach, 276.

Uric acid crystals, 189.

Urine, 189.

Urine, scanty, 154.

Van Helmont, 124.

Vapor bath, 298.

Veal, brofled, time of digestion of, 40.

Veal, fried, time of digestion of, 40.

Veal, nutritive value of, 42.

Vegetable fats, 101.

Vegetable foods, 37 .

Vegetables, 38.

Vegetables and legumes, 232.

Vegetables, raw, 97.

Venison, brofled, time of digestlon of, 40.

Vibrating chair, 335.

VIIII, 28.

VInegar, 34, 109, 112.

Vinegar Bitters, 347.

Viscera, replacement of, 307 .

Vomiting, 153, 167.

Walnut, nutritive value of, 42.

Water-brash, 98, 165.

Water-drinking, 338.

Water, hard, 118.

Water, impure, 129.

Weight, 172.

Wet-sheet pack, 293.

Wheat, Japanese, nutritive value of, 41.

Wheat, Michigan, Diehle, nutritive value of, 41 .

Wheat, Michigan, white, nutritive value of. 41.

Wheat, Poland, nutritive value of, 41.

Wheat flour, 92 .

Wheat meal or bread, 93.

Whortleberry, nutritive value of, 41. 
368

THE STOMACH.

Worcestershire sauce, 34.

Worms, 153.

Worm teas, 127.

Worry, 124.
Yeast, 159.

Zwieback, 240.

Zwieback, nutritive value of, 11. 


\section{THE ART OF MASSAGE.}

By J. H. KELlOGG, M. D.

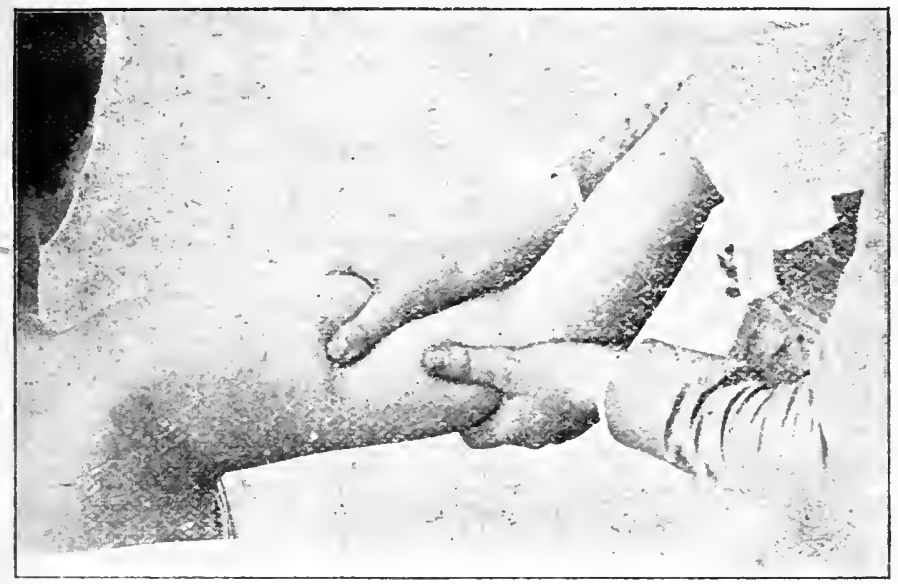

The most complete and thorough work upon the subject of massage ever published. Illustrated by 139 cuts and 10 finely colored anatom= ical plates. Concise directions are given for all the different procedures of scientific massage. It ought to be in the hands of every nurse.

\section{The Perfection Vaporizer.}

THE ONLY PERFECT INSTRUMENT FOR TREATING THE NOSE, THROAT, AND LUNGS.

TS especially adapted to the treatment of diseases of the nose, throat, ear, bronchial tubes, and lungs, both acute and chronic. Many severe spells of sickness can be avoided by its early use. Is especialy recommended in "La Grippe," when affecting the air passages, hay fever, and asthma.

Full directions and formulæ with each instrument.

PRICE, BY EXPRESS, \$3. POSTAGE, 35C., AT'PURCHASER'S RISK.

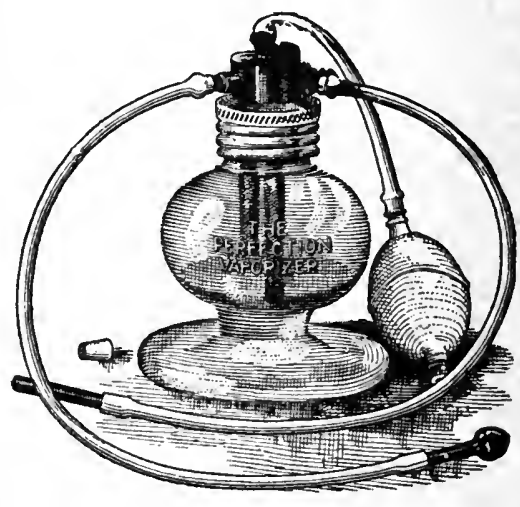

MODERN MEDiCine CO., Battle Creek, Mich. 
The accompanying cut represents these outline Charts mounted on a convenient Exhibitor, which is so arranged that both the Charts and the Exhibitor can be snugly packed in a compact case.

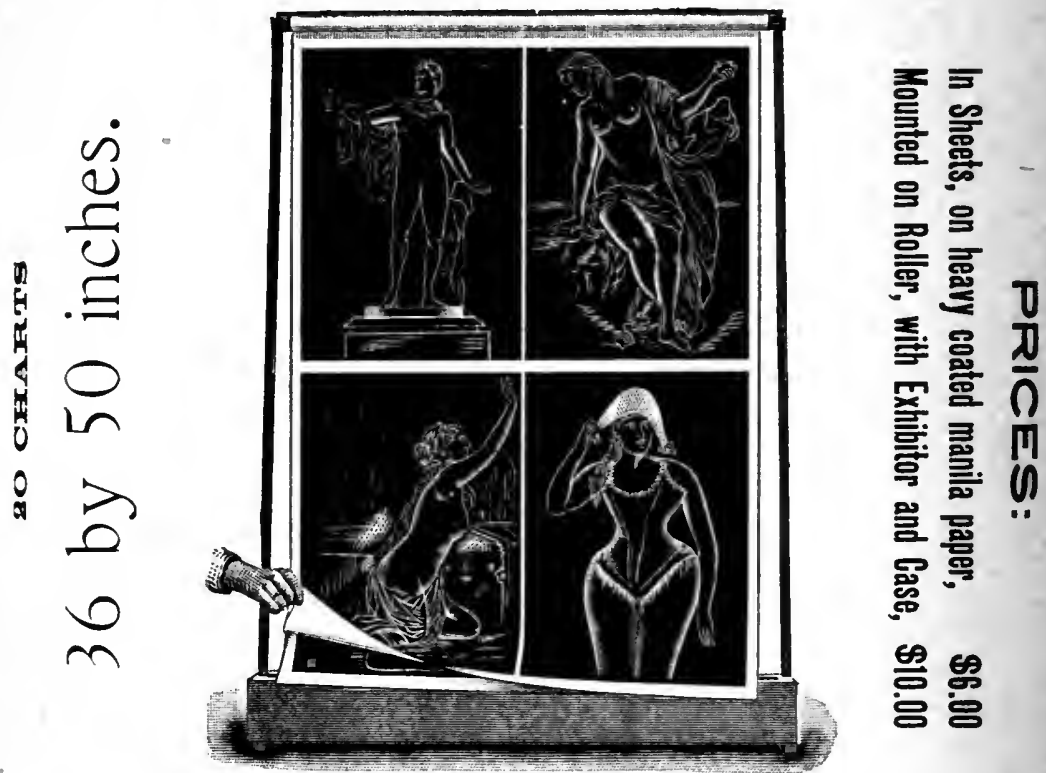

THE following expression regarding the value of Dr. Kellogg's "Outline Studies of the Human Body," is from Jay W. Seaver, A. M., M. D., President of the Chautauqua School of Physical Education, and Medical Director of the Yale University Gymnasium:-

“Dr. Kellogg's 'Outline Studies' I am sure will prove to be very helpful to any person who is studying the human body, or who is teaching personal hygiene. These outlines should be widely introduced into public schools, where their mere presence on the walls would be a constant object lesson."

\section{MODERN MEBICINE PUBLISHING C๐.,} BATTLE CREEK, MICH. 


\section{Something Good to aat, and sollething GoOd Easystigigest

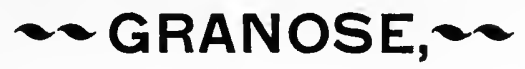

A new cereal preparation made from the choicest wheat, by a process which retains all the elements of the grain. By combining the processes of digestion, cooking, and roasting, by the use of special machinery, the wheat is brought into the form of delicate flakes, in which the bran is thoroughly disintegrated, and the starch largely converted into dextrine, and thus made ready for solution by the digestive juice and for prompt assimilation.

\section{GRANOSE -}

Is crisp, delicious, appetizing, and digests quicker than any other cereal preparation. It clears off the tongue, rids the stomach of germs, and cures constipation.

It is unique; an incomparable food. Bables thrive upon it.

\section{BATTLE GREEK SANITARIUM HEALTH FOOD G0.,}

Battle Creek, Mich.

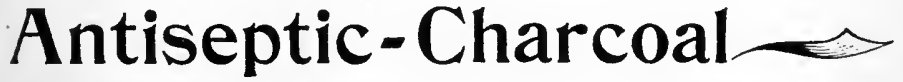 \\ Tablets. -... These Tablets consis of a newly pro. duced form of vegetable charcoal,} which has been shown by experience to be possessed of superior qualities, with which are combined vegetable digestive agents and intestinal antiseptics and antiferments. In these Tablets charcoal is for the first time presented in agreeable form.

\section{When to be Used.}

The use of these Tablets is indicated in all cases of Stomach and Intestinal !ndigestion, and especially in cases in which the following symptoms are present: Sour Stomach, or Acid Fermentation, Bloating of the Stomach or Bowels, Flatulence, Eructations of Gas, Foul Tongue, Bad Breath, Unpleasant Taste in the Mouth, Biliousness, Sick Headache, Nervous Headache, and Constipation.

Dose: One to four Tablets after each meal.

\section{MODERN MEDIGINE 60., Battle Greek, MIoh.}


This preparation is made from carefully selected and prepared nuts. It represents one of the most nourishing and digestible of all the food products of the vegetable kingdom. It is especially adapted for patients who have difficulty in digesting starch, and for those who need to make a rapid gain in flesh. It agrees well with the most delicate stomachs, and will often be digested when the stomach will tolerate nothing else.

Nut Meal may be eaten dry, or combined with other foods, or mixed with a little hot water, when, with the addition of a little salt, it makes a delicious soup or puree.

\section{Nut Butter.}

A capital substitute for animal fats of all sorts in the seasoning or shortening of foods, in the preparation of grae vies, sauces, etc. Thoroughly cooked and emulsified, so it dissolves readily in water; has a rich nutty flavor; is exceedingly palatable and digestible, keeps well, is thoroughly sterilized, and free from all objections which can be urged against animal fats. It gives a meaty flavor to soups.

\section{Almond Meal.}

This is simply a fine meal prepared from the cholcest blanched almonds, especially designed for diabetics and invalids who cannot digest starch. It is highly nutritious, exceedingly delicate and palatable, and an admirable food for tlose who need to make a gain in flesh.

\section{Malted Gluten}

furnishes the farinaceous food elements in a state of complete digestion, READY FOR IM MEDIATE ABSORPTION. The gluten which it contains has been subjected to malt digestion, and is in a state of fine division, so that it is promptly acted upon by the digestive fluids. Gluten is of all food elements the only one which is capable of sustaining life indefinitely. It will thus be seen that MALTED GLUTEN IS A PERFECT BLOOD AND FLESH-MAKINO FOOD. It is free from the unpleasant flavor of the varions meat peptones, and is especially adapted to those cases requiring perfect intestinal asepsis, in which meat peptones and meat preparations of every description are con. tra-indicated. It lias proved a sovereign remedy in cases of nervous headache, sick headache, obstinate nausea, and vomiting, and numerous cases in which all other food substances were rejected by the stomach.

\section{Bromose.}

Are you thin? Have you hollow cheeks, hollow eyes, and a general emaclated appearance? WOULD YOU LIKE TO BE FAT? We will tell you how: EAT BROTOSEI

BROMOSE, an exceedingly palatable food preparation, consists of cereals and nuts, in which the starch is completely digested, the nuts perfectly cooked, and their fat emulsified. It is thus ready for immediate assimila. tion. It is the most easily digested and most fattening of all foods, and at the same time rich in proteids, and hence UN!EQUALED AS A TISSUE BUILDER.

BROMOSE makes fat and blood more rapidly than any other food. It is the food par excellence for blood, brain, and nerves. Invalids whose troubles are due to the fact that they cannot digest the starch of cereals and vegetables, find in BROMOSE A PANACEA. Bromose is rich in salts, as well as proteids and food elements. It is excellent for weak, emaciated invallds, and feeble children. 


\section{Sanitarium

\section{THE ADVANTAGES CLAMMED FOR THIS BATTERY ARE}

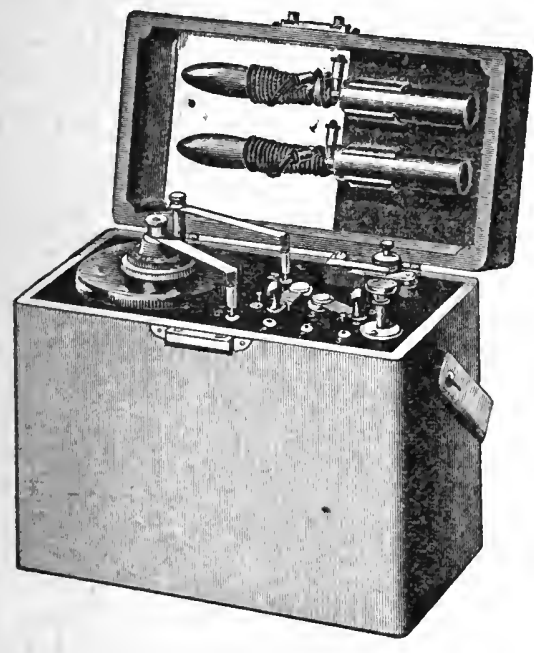

Efficiency, Durability,

Simplicity of Construction,

Ease of Management,

Cleanliness, and lastly,

Small Cost of Maintenance.

\section{. THE CELLS HAVE THE FOL- LOWING ADVANTAGES.}

The elements are zinc and carbon.

The excitant is a solution of muriate of ammonia (sal ammoniac) and water.

There are no fumes nor strong acids to corrode battery parts.

They will run for medical purposes many months without the slightest attention.

There is no consumption of the zinc element when battery is at rest. They are perfectly sealed, so that evaporation is impossible.

High electro-motive force. Small internal resistance. Great power of recuperation.

PRICE, WITH ELECTRODES, COMPLETE, \$10.00. 


\section{COMBINATION}

\section{Water Bottle and Fountain Syringe.}

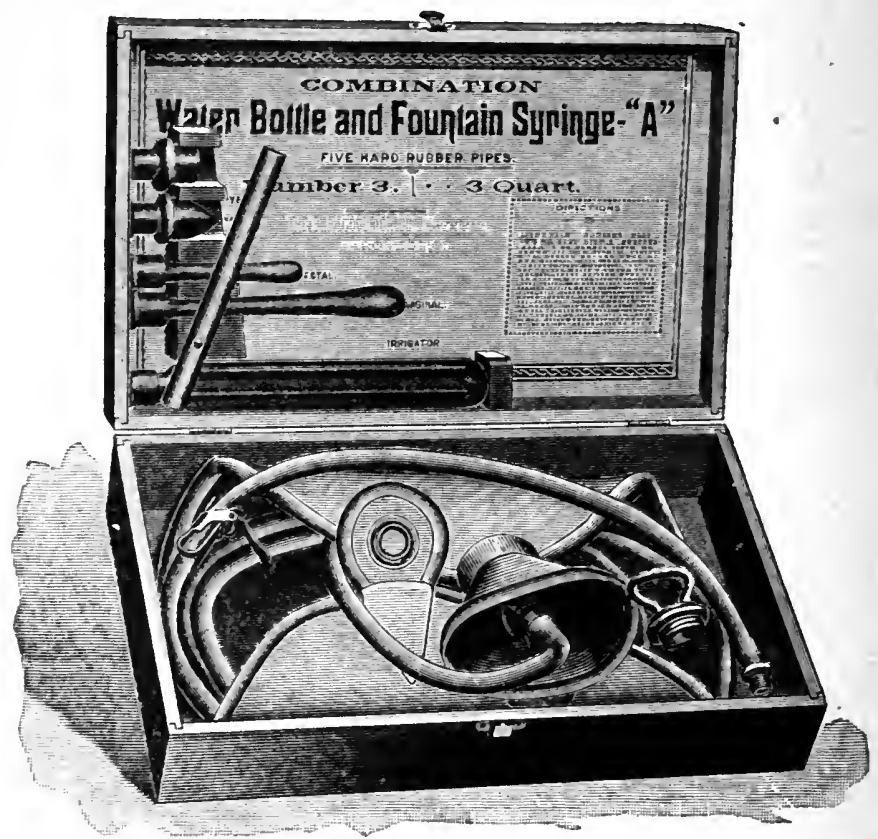

This is a first-class Fountain Syringe, which may be quickly changed to a Water Bottle by detaching the tubing and substituting the metal cap.

This combination was first arranged by us a number of years ago, and we have had hundreds manufactured for us. We have recently arranged for the manufacture, for our special trade, of this convenient combination in an improved form. It consists of a substantial water bottle with fumel neck, a safe and durable cap, and six feet of fine improved tubing, with suitable attachments for connecting with the bag. An outfit of the usual syringe tubes is supplied with the syringe, and the whole is packed in a neat and substantial tin box.

PRICE, complete, $\$ 2.00$.

Postage, 25 cents.

MODERN MEDiCine CO., Battle Creek, Mich. 


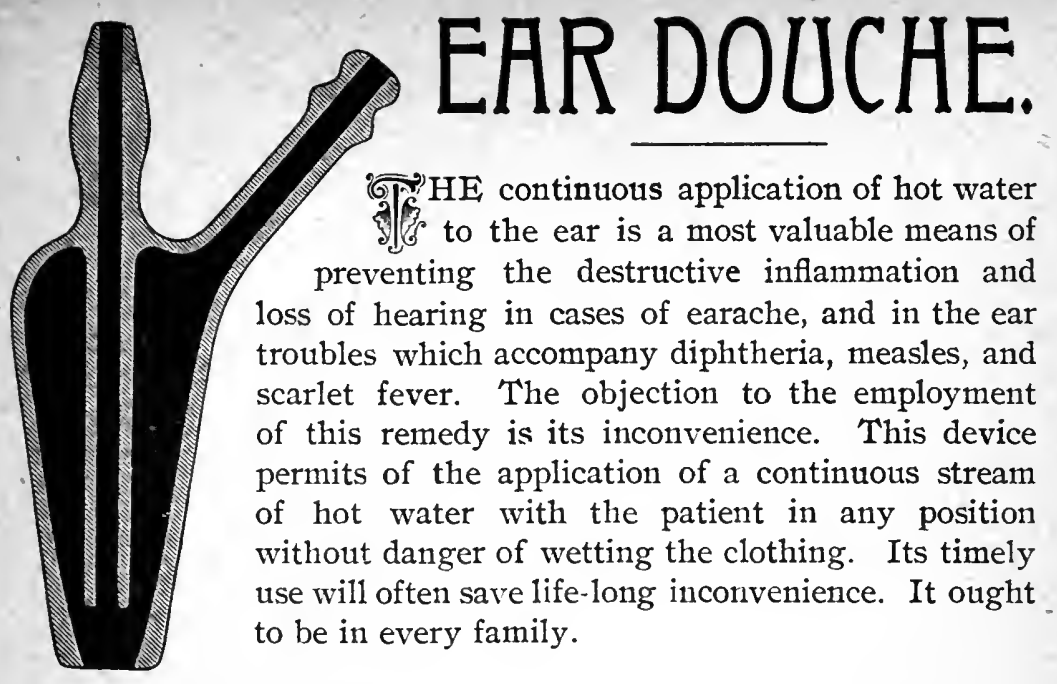

\section{SPINE BAGS.}

RUBBER

SPINE

BAGS.

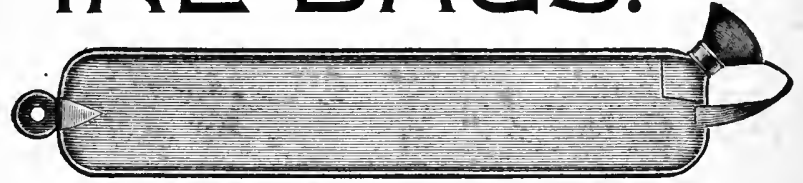

Very strong and durable ; essential in the treatment of some forms of Dyspepsia, Spinal Irritation, and many nervous diseases.

PRICE, 20 inch, post=paid, $==\quad=\$ 1.40$ 26 inch, post=paid, $==\mathbf{1 . 6 5}$

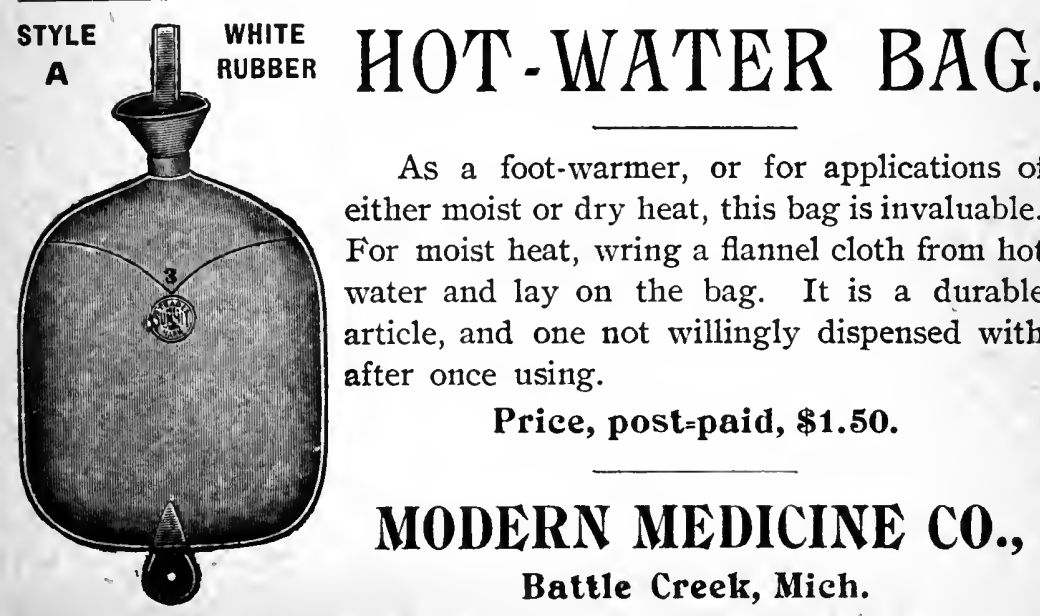




\section{Uulcanized TRubber $\mathbb{T}$ (e JBags.}

THESE bags are designed for making applications of ice to the head, throat, or spine. Where ice cannot be obtained, very cold water may be used. It avoids the necessity for wet cloths, which wet the patient's clothing, soil the bedding, and require constant renewal. The mode of fastening renders the bags water-tight.

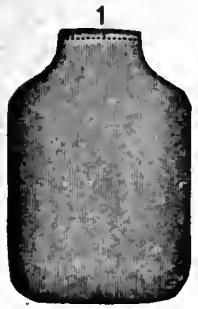

PRICES.

No. $1, \ldots \ldots \ldots \ldots \ldots \ldots$. cents.

No. 2 (narrower than No. I), specially adapted to the head, 50 cents.

No. 3. Specially adapted to the throat. Made of pure gum, and furnished at the following prices:-

7 inches long, .............

8 inches long, ............60 cents.

9 inches long, . . . . . . . .

I I inches long...............

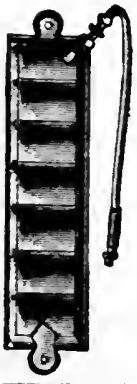

Postage, each, 4 cents.

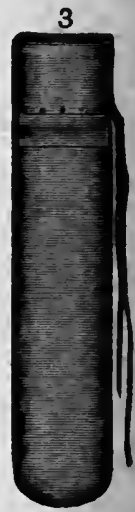

\section{HEAD BAGS.}

Beneficial results may often be obtained by the use of one of these bags in cases of headache. They are very light, and can be filled witl either hot or cold water. They are easily carried and readily applied.

Price, \$2.00. Postage, 5 cents.

Instrument for the Application of Heat to the Vagina.

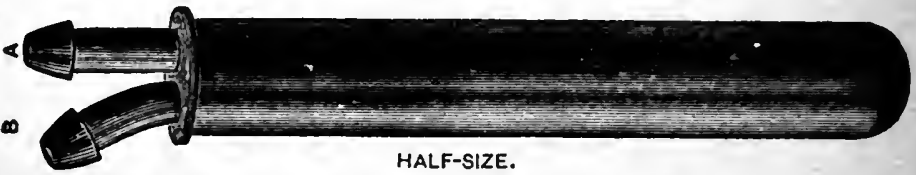

The instrument above shown has been devised for the purpose of applying dry heat to the vaginal walls. It may also be used for cold applications, or alternate hot and cold applications to this region. It is used in connection with a fountain syringe, a current being passed through it either hot, cold, or alternately hot and cold.

PRICES: No. I, \$1.25; No. 2, \$I.00; No. 3, 75 cents. 


\section{THE NATURAL ABDOMINAL SUPPORTER.}

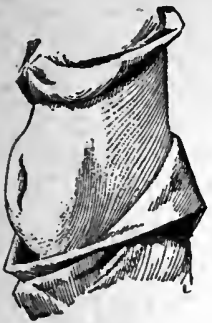

FIG. I.
Ought to be worn by every

woman who has the

shape of Fig. I.

Cures backache, headache, dragging sensation, and many

other discomforts.

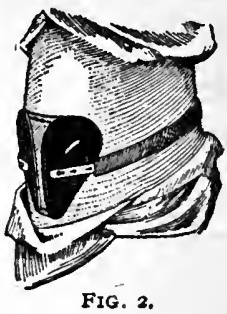

FIG. 2.

PFIOE, POSTPAID, $\$ 5.00$.

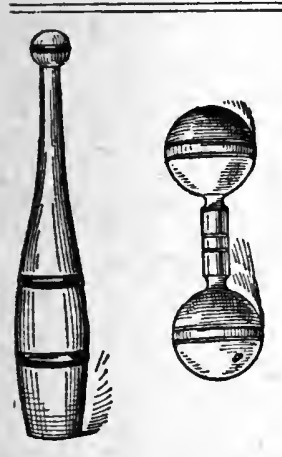

\section{Clubs and Dumb-Bells.}

By the use of these the muscles can be exercised and developed, giving vigor, appetite, and cheerfulness to the user.

SIZES : 1.2, 3-4, 1, 1 1.2, 2, 2 1.2, 3, and 4 lbs.

The $1 / 21 \mathrm{~b}$. is adapted to the use of children from 2 to 4 years of age. The $3 / 4$, from 4 to 8 years of age. Prices given on application.

We furnish outfits for home gymnasiums at various prices from \$IO.00 upwards. One of these outfits ought to be in every home.

\section{SEND FOR CIRCULAR AND PRICES.}

\section{MUSCLE BEATERS.}

Simple, cheap, and efficient instruments for securing some of the effects of massage. By their habitual use one can obtain most beneficial results without the aid of an expert.

Price of Ball Beater, postpaid, - $\$ 1.40$

Price of 4-finger Beater, postpaid, 2.15

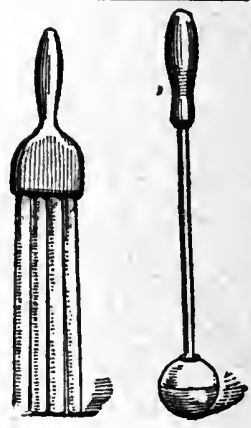

MOdeRn medicine CO., Battle Creek, Mich. 


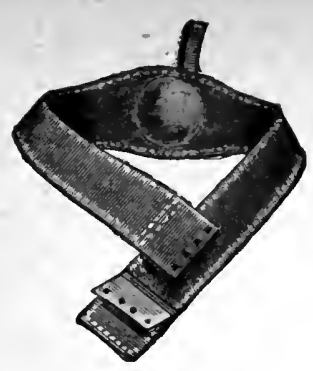

\section{Umbilical Belts.}

Made to order of any size desired.

PRICE, \$r.5o. Postage, 4 cents.

Air Pillows are always cool and restful. They can be adjusted at will. Just the thing for camping out or traveling. Can be packed in small space by letting the air out.

\section{PRICE,}

No. I, Sateen cover, $9 \times 13, \quad \$ 1.60$

No. 3, " " $\quad 12 \times 18, \quad 2.50$

Postage, I 5 cents.
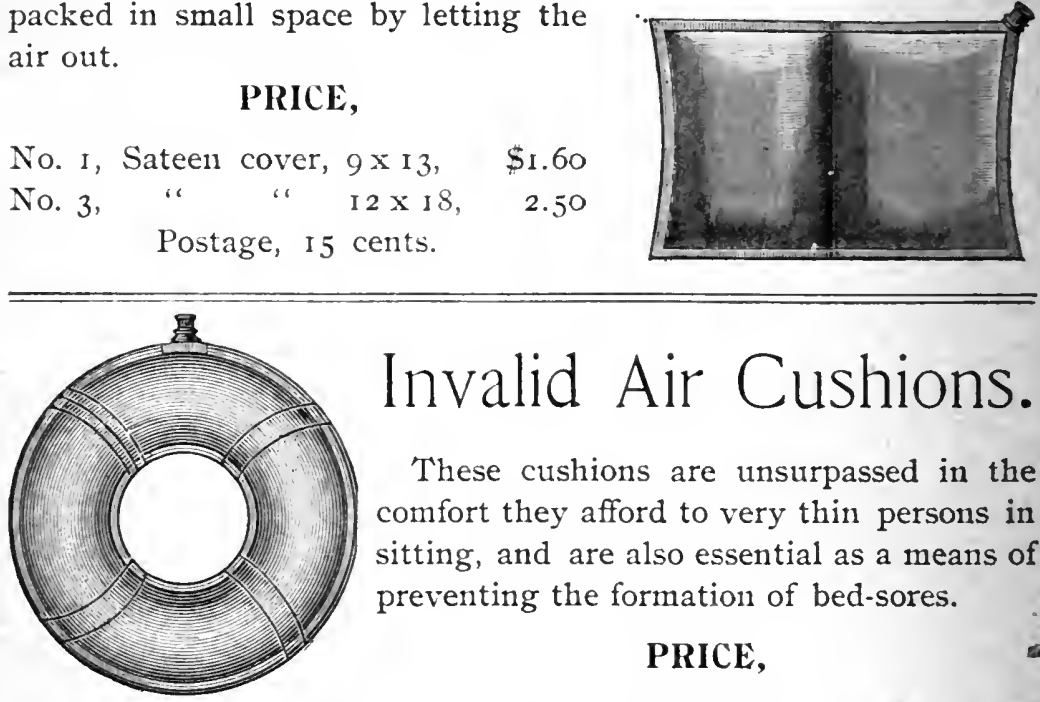

\section{Invalid Air Cushions.}

These cushions are unsurpassed in the comfort they afford to very thin persons in sitting, and are also essential as a means of preventing the formation of bed-sores.

PRICE,

9 inches in diameter (postage, ro cents), - $\$ 1.60$

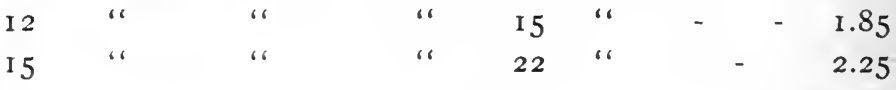

\section{Exhalation Tube for Lung Development.}

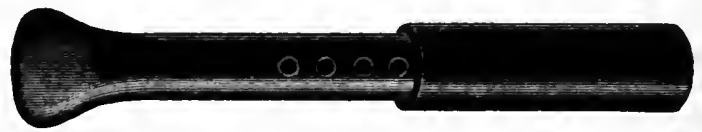

The Exhalation Tube is one of the most effective means for lung development. It can be carried in the pocket, and can be used at any time without interfering with other occupations, and can thus be made to act continuously in developing the lungs, expanding the air-cells, and deepening the respiratory movements. It is worth many times its weight in gold to a consumptive, or to any other person whose lungs are weak.

MODERN MEDICINE CO., Battle Creek, Mich. 


\section{PERFECTION}

\section{DOUCHE APPARATUS}

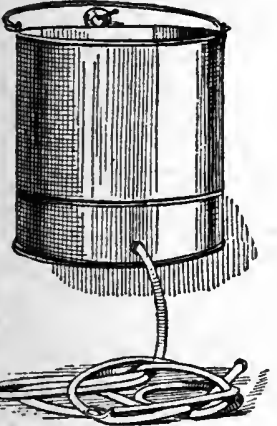

THIS apparatus consists of a pail for water, with a long rubber tube, and a convenient bed=pan. It is made of tin, and is light, durable, and easily cleaned and disinfected.

It is especially useful for giving douches in cases of confinement, and to feeble patients who cannot be removed from the bed.

It can be adjusted under the hips without moving the patient, on any form of bed or mattress.

Any amount of water can be used, the water running out as fast as it runs in. To start the water running, close the rubber tube by folding it below the bulb, and then squeeze the bulb, which will at once fill with water when

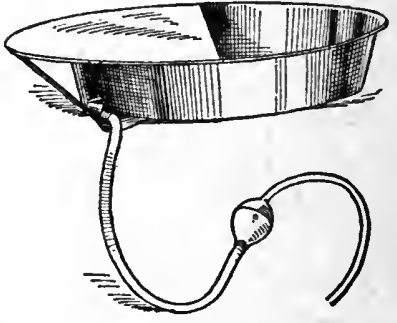
released. Then open the tube, and the stream will continue to flow till the pan is empty.

\section{PRICE,}

Complete, . . . . . . . . . . . . \$2.25.

Douche Pan, without fountain or tubes, . I.25.

Sent by express.

MODERN MEDiCine CO., Battle Creek, Mich. 
IfHE great prevalence of diseases of the rectum, and the recognized advantages 1 years ago, to the invention of the hard rubber rectal irrigator, of which the inventor speaks as follows :-

"The virtue of hot water as an agent in removing the thickenings and indurations which result from chronic congestions and inflammations of mucous surfaces and con-

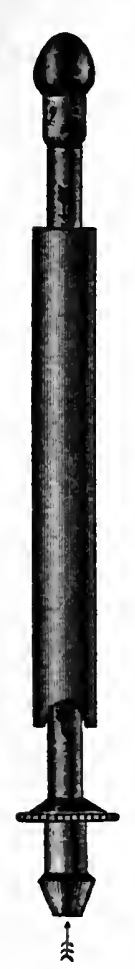
tiguous parts, has long been recognized. In the treatment of pelvic indurations resulting from cellulitis, no agent is so potent as the hot water vaginal douche. Dr. Emmett states that he regards this one agent as more valuable than all other local measures combined. The value of hot water in the treatment of rectal diseases seems to have been less appreciated by the profession, though recently it has been used by various specialists, and with excellent results.

"For some years I have used hot water by means of the continuous enema, and with most excellent results; but feeling the need of some better mode of applying hot water to this part of the body, I have experimented with various forms of instruments for the purpose. All the instruments I found in use were too large to be used without discomfort, in most cases, and all were made of metal, which is wholly unfit for this purpose on account of its great conductivity of heat. The mucous membrane is much more tolerant of heat than is the skin. When a metallic instrument is used, the heat transmitted to the very sensitive surface at the junction of the skin and mucous membrane becomes unbearable before the temperature is high enough to make the application of much value. I have had made an instrument, which I believe embodies the excellencies of instruments previously made, and several additional advantages, chiefly the following:-

" 1 . It is made of hard rubber, a poor conductor of heat, which allows the use of water as hot as the nucous membrane will tolerate.

" 2 . The instrument is of such size that it can be easily used even in those cases in which there is unnatural contraction of the sphincter muscles from the irritation of a rectal ulcer or fissure, the class of cases in which an instrument of this sort is of greatest service, and in which other in-, struments can seldom be used, never with water of proper temperature.

" 3 . The sleeve of the instrument is notched at its outer extremity so as to prevent obstruction to the return flow when the inner tube is pushed in as far as is allowed by the stop.

"I have used this instrument in the treatment of hundreds of cases of rectal diseases of various sorts, and have found it a most satisfactory and effective aid in the management of this class of diseases."

\section{CAN BE USED WITH ANY SYRINGE.}

Directions for use accompany each Instrument.

PBICX, postage paid, $\$ 1.00$. Fith Cnirersal Syphon Syringe, $\$ 2.25$; postage 15 ets. additional. 


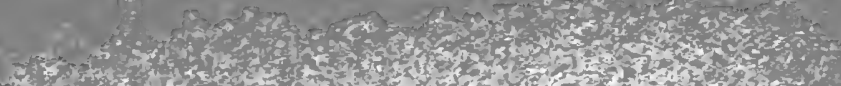

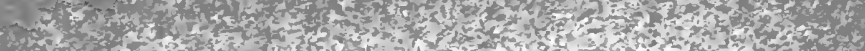

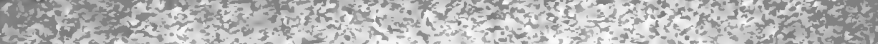

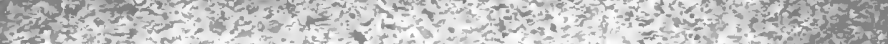

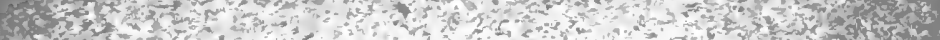

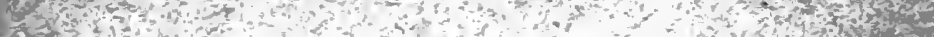

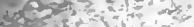

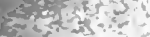
rex

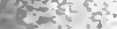

$0+x^{2}=-x=8$

$+2=$

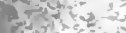

s?

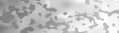

bre 1 is

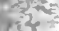

तर,

ant

कixisicir

ies

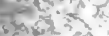

$7 x^{2}-2=2$

$\rightarrow$

$+x^{2}+x^{2}=1+1$

extat

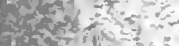

ind :4

ats $1 x^{2}$

$=0+3<2$

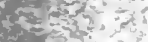

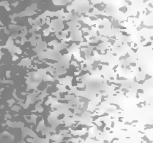

fhes

afin

atr. $=$

get $0-3$

$x^{2}-x^{2}+2$

$4=x+5$

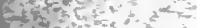

3. $x+20,13=0$

axtsid $=$

कर $=$

ians

tosicis

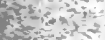

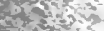

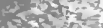

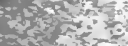

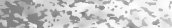

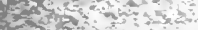

52 .

$45 x-3, \frac{1}{2}$

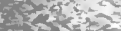

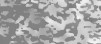

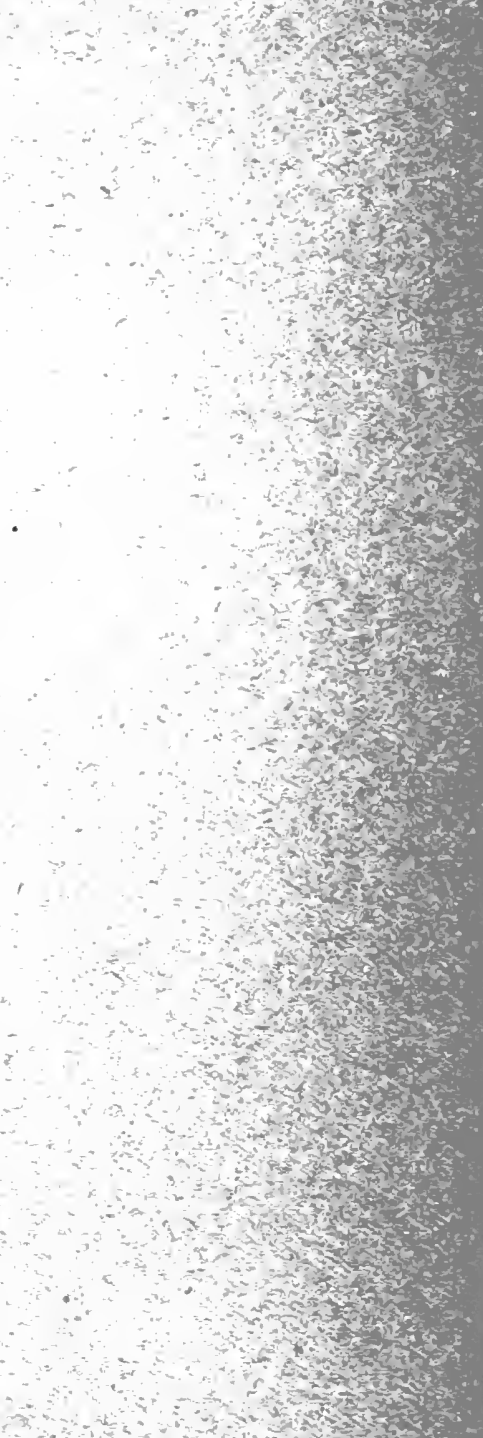

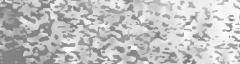

thes

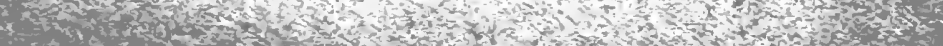

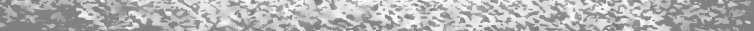




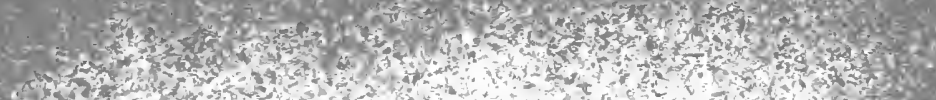

3 $-100,0$

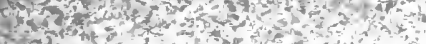
10 -2 ents te. 6 (3hes

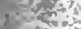
$-7 x^{5}+x^{2}-4$

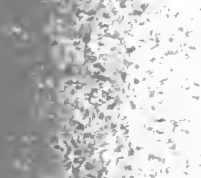

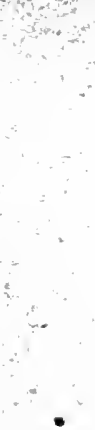

5 int

- 3 ise ints

atman

$\frac{17}{2}+x^{2}$

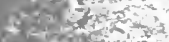

\& 0 .

dontion

the

vesicists

ares $x=$

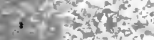

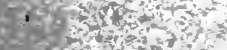

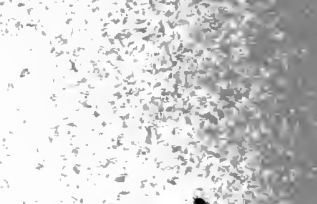

$\therefore-10$ $-x^{2}+3 x$

$-$

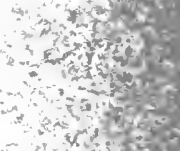

$\therefore=2+x^{2}$ ind istest initions 0 int $x^{2}=$ 4ent $\therefore+5425$ tuens $3 \times 2,100$ $+2,35$

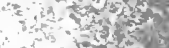
$i^{2}+2 x+20$ $1+x=250$ -5 u. intes $\lim ^{2} x-25$ a $\therefore$ and $-\infty$ ints w...t.

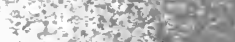
w $1+\sin ^{2} \mathrm{x}$

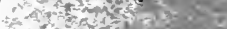

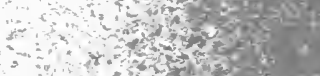

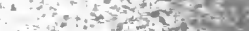
$\therefore y^{-1}+2 x$

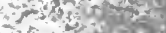

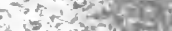

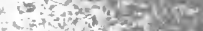

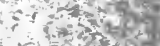

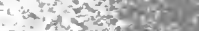

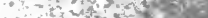
?

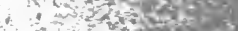

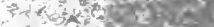
$\because x-2 x^{2} x^{2} x$

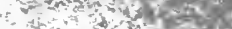
$-2+3 x+1$

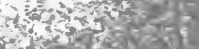

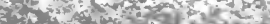
$2 x+2=12$ 


\section{Date nus}

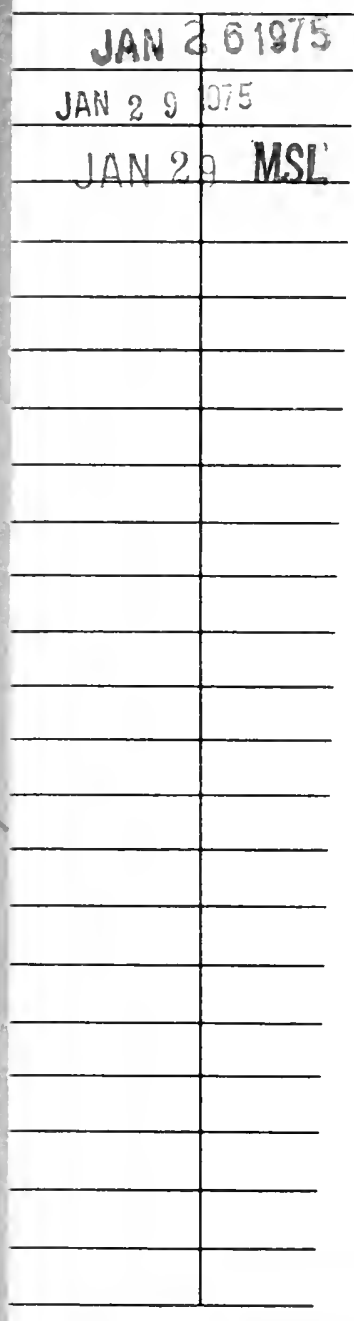

University of California

SOUTHERN REGIONAL LIBRARY FACILITY

405 Hilgard Avenue, Los Angeles, CA 90024-1388

Return this materlal to the library

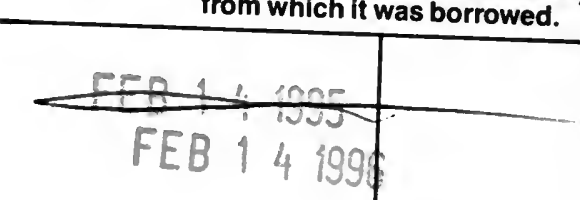

APR 262007

PRINTEOIN U.S.A. CAT. NO. 24161

BN 
Kellogg, John H

WI 113

K29s

1896

The stomach

Kellogg, John H

\section{WI 113}

K29s

1896

The stomach

\section{MEDICAL SCIENCES LIBRARY \\ UNIVERSITY OF CALIFORNIA, IRVINE \\ IRVINE, CALIFORNIA 92664}


